\title{
Reactions at Supercritical Conditions: Applications and Fundamentals
}

\author{
Phillip E. Savage, Sudhama Gopalan, Tahmid I. Mizan, Christopher J. Martino, and Eric E. Brock \\ Chemical Engineering Dept., University of Michigan, Ann Arbor, MI 48109
}

\begin{abstract}
Supercritical fluids possess properties that make them attractive as media for chemical reactions. Conducting chemical reactions at supercritical conditions affords opportunities to manipulate the reaction environment (solvent properties) by manipulating pressure, to enhance the solubilities of reactants and products, to eliminate interphase transport limitations on reaction rates, and to integrate reaction and separation unit operations. Supercritical conditions may be advantageous for reactions involved in fuels processing, biomass conversion, biocatalysis, homogeneous and heterogeneous catalysis, environmental control, polymerization, materials synthesis, and chemical synthesis. Moreover, supercritical fluids can be used profitably in fundamental chemical investigations of intermolecular interactions and their influence on chemical processes. Work on chemical reactions in and with supercritical fluids is reviewed. We discuss both fundamental studies and applications of reactions at supercritical conditions, with focus on work published after 1985.
\end{abstract}

\section{Introduction}

The critical point of a fluid marks the terminus of the vapor-liquid coexistence curve. At temperatures above the critical temperature $\left(T_{c}\right)$ a fluid cannot undergo a transition to a liquid phase, regardless of the pressure applied. A fluid is said to be "supercritical" when its temperature and pressure exceed the temperature and pressure at the critical point. Table 1 lists the critical temperature and pressure for different fluids that have been used in reaction studies at supercritical (SC) conditions. The data were taken from Reid et al. (1977).

$\mathrm{CO}_{2}$ and water are the fluids used most frequently in reactions at supercritical conditions. $\mathrm{CO}_{2}$, which has a critical temperature near ambient conditions, has often been used because of this experimental convenience. More recently, however, $\mathrm{SC} \mathrm{CO}_{2}$ has begun to attract attention as an environmentally benign substitute for some halogenated and aromatic solvents used in chemical syntheses (DeSimone et al., 1992; Reetz et al., 1993; Tanko and Blackert, 1994). Water, which possesses a much higher critical temperature and pressure, has been used frequently in reaction studies because of potential practical applications of reactive processes at supercritical conditions that employ water.

Correspondence concerning this article should be addressed to P. E. Savage
Supercritical fluids (SCFs) are attractive media for chemical reactions because of their unique properties. Many of the physical and transport properties of an SCF are intermediate between those of a liquid or a gas. Table 2 shows representative, order of magnitude values for some SCF properties relevant to chemical reactions (Squires et al., 1983; Tiltscher and Hofmann, 1987). The diffusivity in an SCF falling between that in a liquid and a gas suggests that reactions that are diffusion limited in the liquid phase could become faster in an SCF phase. For example, the rate of a liquid-phase enzyme-catalyzed reaction can be enhanced by performing the reaction in $\mathrm{SC} \mathrm{CO}_{2}$ (Russell and Beckman, 1991b).

SCFs also have unique solubility properties. Compounds that are largely insoluble in a fluid at ambient conditions can become soluble in the fluid at supercritical conditions. Conversely, some compounds that are soluble at ambient conditions can become less soluble at supercritical conditions. Figure 1 shows experimental data for naphthalene solubility in $\mathrm{CO}_{2}$ (McHugh and Paulaitis, 1980) and salt solubility in water (Tester et al., 1993) that illustrate this effect. A good example of the use of solubility effects in SCF reaction processes is the development of $\mathrm{SC}$ water oxidation processes for treating organic wastes (Modell, 1989). SC water enjoys a high solubility for organics and a reduced solubility for salts rela- 
Table 1. Critical Properties of Selected Fluids

\begin{tabular}{lcc}
\hline Fluid & $T_{c}(\mathrm{~K})$ & $P_{c}(\mathrm{~atm})$ \\
\hline Ethylene & 282.4 & 49.7 \\
Xenon & 289.7 & 57.6 \\
Trifluoromethane & 299.1 & 48.1 \\
Carbon dioxide & 304.2 & 72.8 \\
Ethane & 305.4 & 48.2 \\
Nitrous oxide & 309.6 & 71.5 \\
Sulfur hexafluoride & 318.7 & 37.1 \\
Propane & 369.8 & 41.9 \\
1,1-Difluoroethane & 386.6 & 44.4 \\
Ammonia & 405.6 & 111.3 \\
Methyl amine & 430.0 & 73.6 \\
1-Hexene & 504.0 & 31.3 \\
$t$-Butanol & 506.2 & 39.2 \\
$n$-Hexane & 507.4 & 29.3 \\
Acetone & 508.1 & 46.4 \\
$i$-Propanol & 508.3 & 47.0 \\
Methanol & 512.6 & 79.9 \\
Ethanol & 516.2 & 63.0 \\
Toluene & 591.7 & 40.6 \\
$p$-Xylene & 616.2 & 34.7 \\
Water & 647.3 & 217.6 \\
Tetralin & 719.0 & 34.7 \\
\hline
\end{tabular}

tive to water at ambient conditions. Another example of enhanced solubility can be found in homogeneous catalysis and organometallic chemistry where reactions of organometallic compounds with permanent gases occur. These gases (e.g., $\mathrm{H}_{2}$ ) are sparingly soluble in liquid organic solvents, but they can be present in much higher concentrations in SCF solvents. This enhanced solubility can accelerate reaction rates and facilitate the synthesis of novel organometallic compounds. This effect can also be exploited to overcome potential interphase transport limitations, to reduce carbon deposition on heterogeneous catalysts, or to simplify downstream separation and purification of reaction products and unreacted reactants.

One additional attractive feature of SCFs as a medium for chemical reactions is that their properties vary with density, which is a strong function of temperature and pressure in the critical region. Consequently, SCFs provide the opportunity to engineer the reaction environment by manipulating temperature and pressure. SC water, for instance, can be a medium for either ionic or free-radical chemistry, depending on the reaction conditions (Antal et al., 1987a). Furthermore, a solvent's dielectric constant can influence the rates of reactions with a change in polarity along the reaction coordinate. Thus, density can be used as a lever to manipulate the dielectric constant and hence reaction kinetics. As examples of the opportunities that exist to tune reaction rates, equilibrium, and selectivities in SCF reaction media we offer Figures 2-5. Figures 2 and 3 show the effect of pressure on the rate con-

Table 2. Comparison of Typical SCF, Liquid, and Gas Properties

\begin{tabular}{lccc}
\hline & Liquid & SCF & Gas \\
\hline Density $\left(\mathrm{g} / \mathrm{cm}^{3}\right)$ & 1 & $0.1-0.5$ & $10^{-3}$ \\
Viscosity $(\mathrm{Pa} \cdot \mathrm{s})$ & $10^{-3}$ & $10^{-4}-10^{-5}$ & $10^{-5}$ \\
Diffusivity $\left(\mathrm{cm}^{2} / \mathrm{s}\right)$ & $10^{-5}$ & $10^{-3}$ & $10^{-1}$ \\
\hline
\end{tabular}

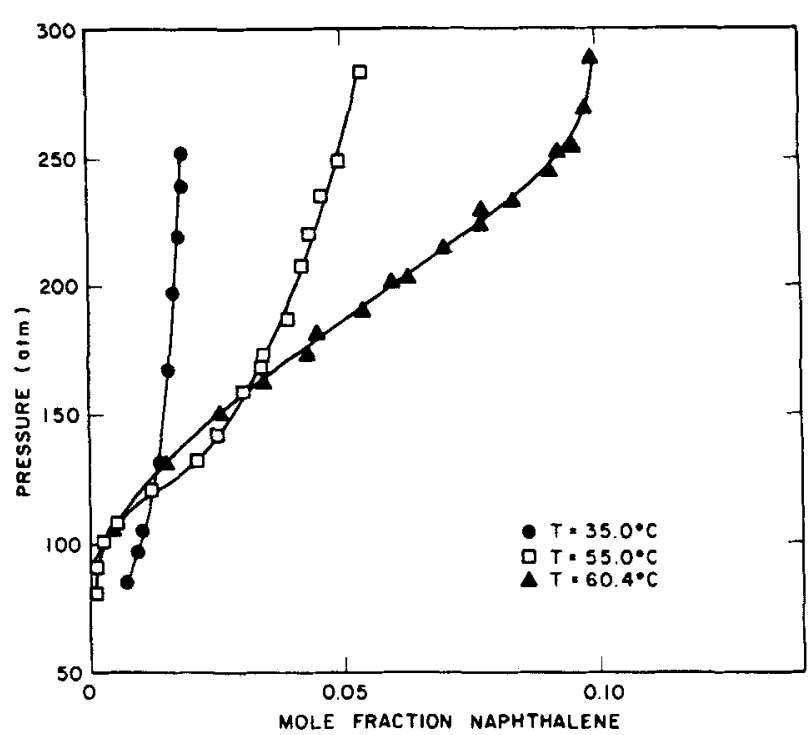

(a)

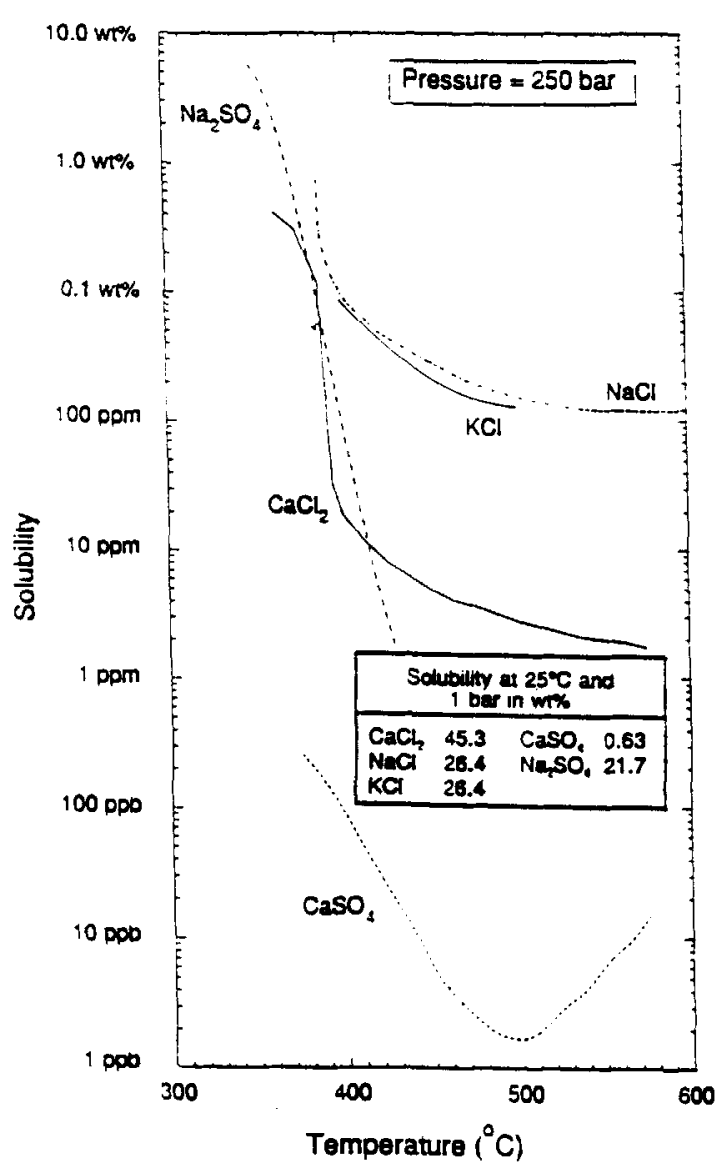

(b)

Figure 1. Solubilities in SCFs.

(a) Effect of pressure on naphthalene solubility in $\mathrm{SC} \mathrm{CO}_{2}$ (McHugh and Paulaitis, 1980). (b) Effect of temperature on salt solubility in $\mathrm{SC}$ water at $25 \mathrm{MPa}$ (Tester et al., 1993). Courtesy of American Chemical Society.

stants for the decomposition of chlorobenzyl methyl ether in SC 1,1-difluoroethane (Johnston and Haynes, 1987) and the Diels-Alder cycloaddition of maleic anhydride and isoprene 


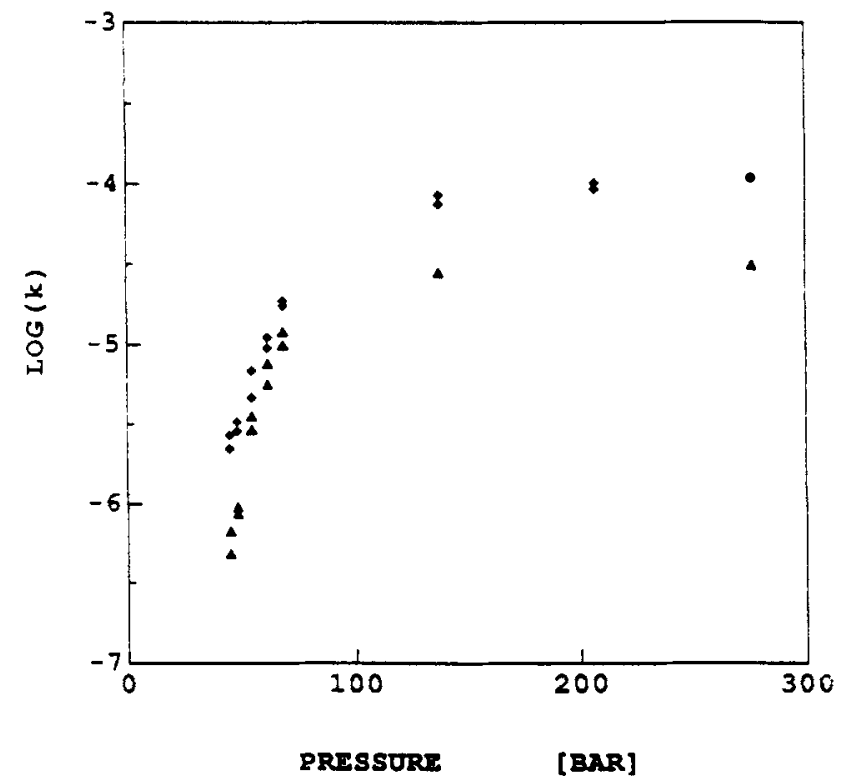

Figure 2. Effect of pressure on unimolecular reaction rate constant.

The reaction is the decomposition of $\alpha$-chlorobenzyl methyl ether in SC 1,1-difluoroethane. Triangles represent data at $403 \mathrm{~K}$, diamonds are at $423 \mathrm{~K}$ (Johnston and Haynes, 1987).

in $\mathrm{SC} \mathrm{CO}_{2}$ (Paulaitis and Alexander, 1987). It is clear that the rate constant is a very strong function of pressure in the critical region and that the effect of pressure is reduced at higher reduced temperatures. Figure 4 shows the effect of pressure on the selectivity to two different photodimers of

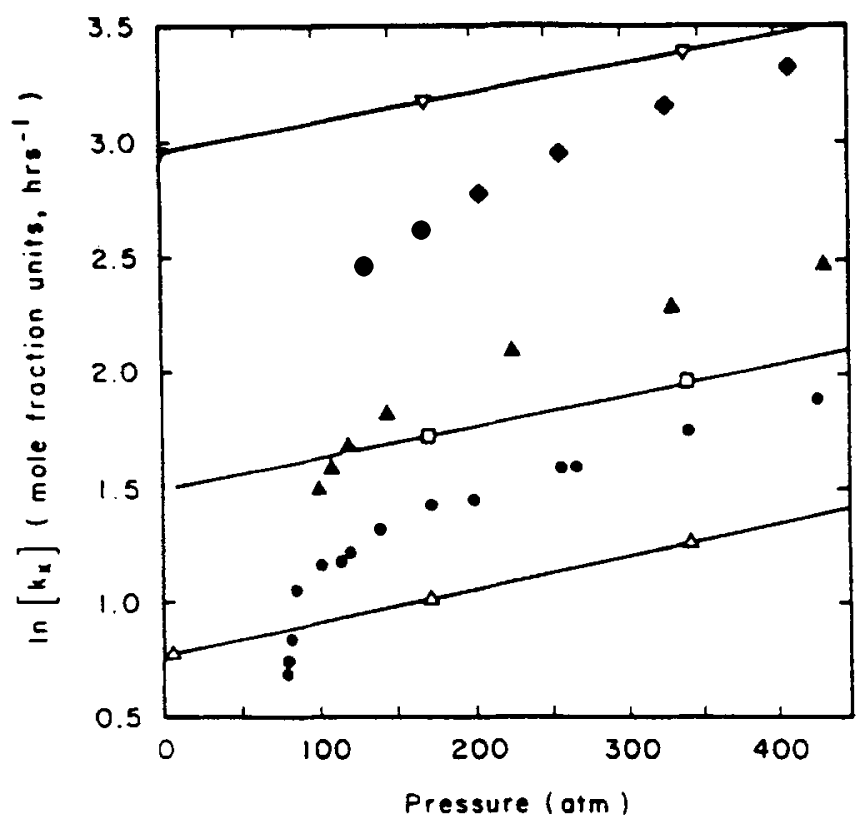

Figure 3. Effect of pressure on bimolecular reaction rate constant

The reaction is the cycloaddition of maleic anhydride and isoprene in $\mathrm{SC} \mathrm{CO}_{2}$. The three sets of data are from experiments at 35,45 , and $60^{\circ} \mathrm{C}$. The lines represent kinetics data at $35^{\circ} \mathrm{C}$ in three different liquid solvents (Paulaitis and Alexander, 1987).

Courtesy of International Union of Pure and Applied Chemistry.

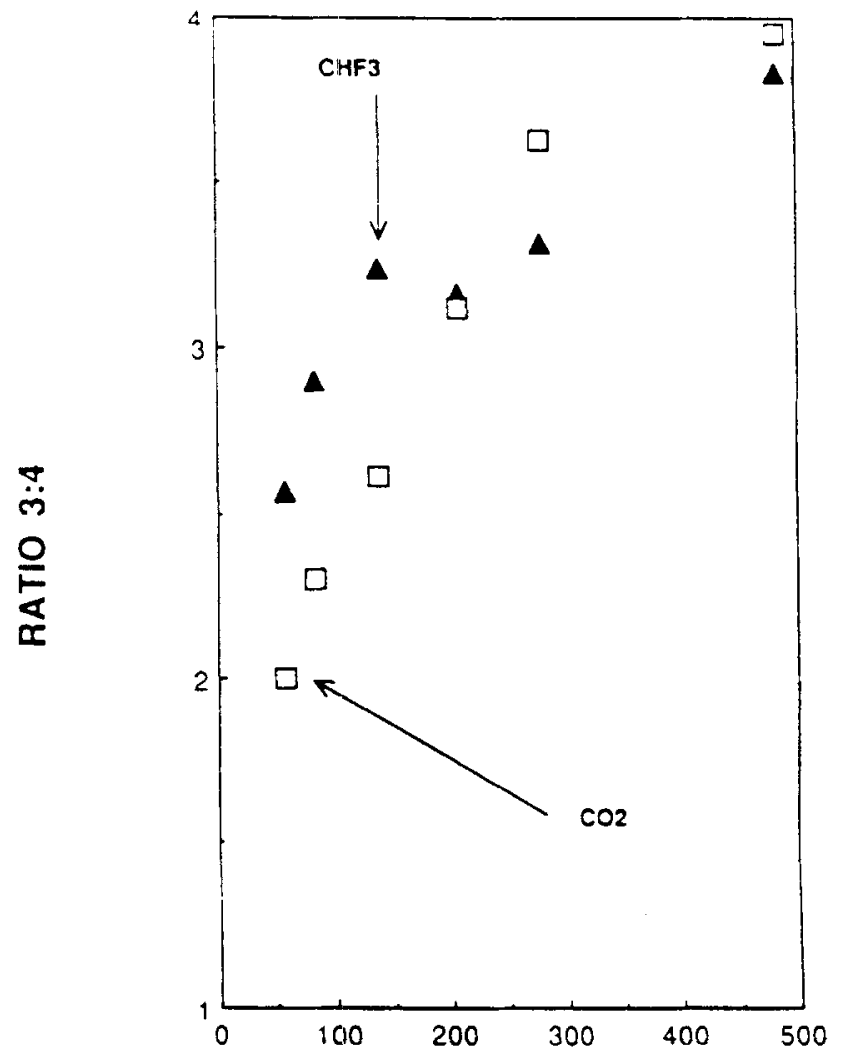

PRESSURE (bar)

Figure 4. Effect of pressure on selectivity.

The reaction is photodimerization of isophorone in $\mathrm{SC} \mathrm{CO}_{2}$ and $\mathrm{CHF}_{3}$, and the selectivity is the ratio of head-to-tail anti to head-to-tail syn photoproducts (Hrnjez et al., 1989). Courtesy of American Chemical Society.

isophorone in $\mathrm{SC} \mathrm{CO}_{2}$ and $\mathrm{CHF}_{3}$ (Hrnjez et al., 1989). Again, a strong pressure effect is evident, which indicates that pressure can influence selectivity for at least some reactions. Finally, Figure 5 gives the effect of pressure on the equilibrium constant for a tautomerization reaction in 1,1-difluoroethane (Peck et al., 1989). Once more, pressure has a strong influence in the region near the critical point.

Sometimes, the properties of an SCF being between those of a liquid or a gas provide multiple reasons for considering SCF reaction media. For example, SCF media may be better than gas- or liquid-phase media for some heterogeneous catalytic reactions (olefin isomerization, Fischer-Tropsch synthesis). The SCF affords a higher diffusivity than does a liquid, and it provides a higher solubility for coke precursors than does a gas. Thus, the SCF can simultaneously offer good catalyst activity maintenance and high effectiveness factors.

Interest in SCFs has experienced explosive growth in recent years, and much of that growth centers around chemical reactions. This strong interest in SCFs and conducting chemical reactions therein has been spurred by potential applications of technologies based on SCFs. These applications, which include homogeneous and heterogeneous catalysis, polymerization, waste treatment technologies, conversion of coal and biomass to fuels and chemicals, combustion, materials synthesis, enzymatic reactions, and electrochemistry, pro- 


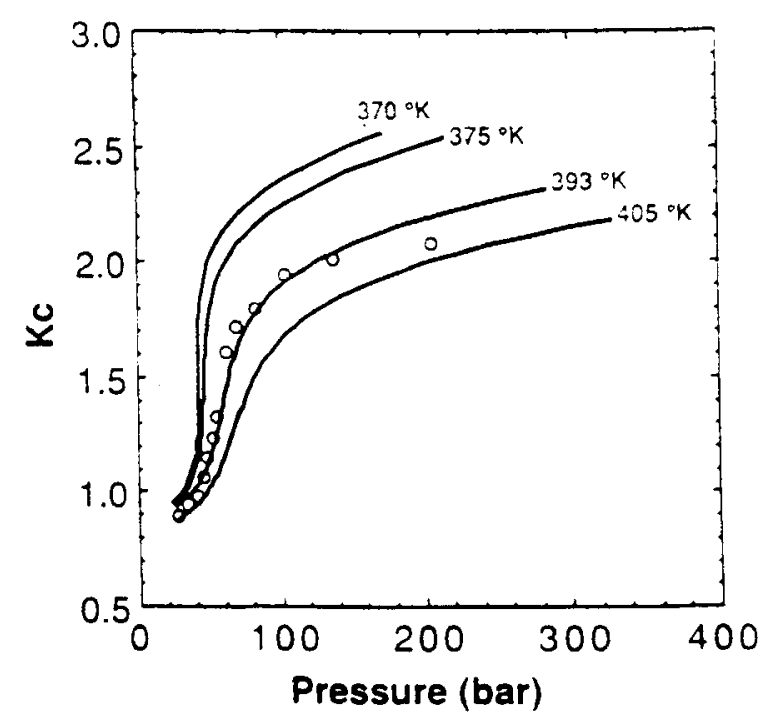

Figure 5. Effect of pressure on equilibrium constant.

The reaction is the 2-hydroxypyridine/2-pyridone tautomerization in SC 1,1-difluoroethane. Open circles are experimental data at $403 \mathrm{~K}$ (Peck et al., 1989).

Courtesy of American Chemical Society.

vide a natural framework for organizing this review. Before beginning this review on reactions at supercritical conditions, we first note other recent reviews of relevant material. Subramaniam and McHugh (1986) reviewed work in reactions in SCFs prior to 1985 , when the field was still in its infancy. Their review provides a good introduction to the field and its history. It also includes a good discussion of high-pressure phase behavior and its importance in SCF systems. We do not duplicate the material covered in this earlier review; rather we focus on the more recent developments in the field. The Subramaniam and McHugh review is complementary to the present review. Additional, though more narrowly focused reviews include a feature article (Shaw et al., 1991) on reactions in SC water, a review of spectroscopic studies of reactions in SCFs (Brennecke, 1993), and brief overviews on reactions in SCFs that highlight the work of Klein's group with SC water (Wu et al., 1991a; Boock et al., 1992, 1993). Although we cite selected portions of the patent literature in this review, we do not include an exhaustive review. Bruno (1991) gives a complete list of SCF-related patents issued between 1982 and 1989. McHugh and Krukonis (1986, 1994) include an appendix with some SCF patent summaries, but these focus on SCF extraction. Finally, some of the applications we discuss have also been the topic of dedicated review articles or have been concisely summarized in a regular research paper. We will refer the reader to these more narrowly focused reviews in the individual applications sections.

\section{Applied Kinetics}

This section gives a brief introduction to and an overview of transition-state theory, pressure effects, and selected solvent effects on chemical reactions. These concepts and tools are used frequently in SCF reaction studiès. This section is not intended to give a thorough and detailed accounting of these subjects. Throughout this section we cite texts and reviews to which the interested reader can turn for more detailed information.

\section{Transition-state theory}

Transition-state theory is a convenient and powerful formalism for explaining and interpreting the kinetics of elementary reactions. This theory views a chemical reaction as occurring via a transition-state species (or an activated complex), which we denote as $M^{\ddagger}$. The superscript double dagger identifies a species or property with the transition state. The transition state is identified as the state of maximum energy along the reaction coordinate, where the reaction coordinate is the minimum energy pathway between the reactants and the products on the potential-energy surface.

To develop and illustrate the basic uses of transition-state theory we will consider the generic elementary reaction

$$
a A+b B+\cdots \leftrightarrow M^{\ddagger} \rightarrow \text { Products. }
$$

The transition-state theory rate constant $(k)$ is given by (Moore and Pearson, 1981)

$$
k=\kappa \frac{k_{B} T}{h} K_{c}^{\ddagger}
$$

where $\kappa$ is the transmission coefficient, $k_{B}$ is the Boltzmann constant, $h$ is Planck's constant, $T$ is absolute temperature, and $K_{c}^{\ddagger}$ is the concentration-based equilibrium constant for the reaction involving the reactants and the transition state. Now, the equilibrium constant accessible from classical thermodynamics, $K_{a}^{\ddagger}$, is related to $K_{c}^{\ddagger}$ as (Sandler, 1989)

$$
K_{c}^{\ddagger}=\frac{K_{a}^{\ddagger}}{K_{\gamma}^{\ddagger}} \rho^{(1-a-b-\ldots)}
$$

where $K_{\gamma}^{\ddagger}=\Pi \gamma_{i}^{\nu_{i}}, \gamma_{i}$ and $\nu_{i}$ are the activity coefficient and stoichiometric coefficient (e.g., $a, b, \ldots$ ), respectively, for component $i$, and $\rho$ is the molar density of the reacting mixture. We can then write the transition-state theory rate constant as

$$
k=\kappa \frac{k_{B} T}{h} \frac{K_{a}^{\ddagger}}{K_{\gamma}^{\ddagger}} \rho^{(1-a-b-\ldots)}
$$

or as

$$
k_{x}=\frac{k}{\rho^{(1-a-b-\ldots)}}=\kappa \frac{k_{B} T}{h} \frac{K_{a}^{\ddagger}}{K_{\gamma}^{\ddagger}},
$$

if concentration-independent units (e.g., mole fraction) are desired for the rate constant. One could also develop an alternate expression for the transition-state theory rate constant that employs fugacity coefficients rather than activity coefficients (Eckert et al., 1974). This alternate form of the rate constant is convenient to use when an accurate analytical equation of state is available for the fluid phase.

The rate constant in Eq. 5 can be rewritten as

$$
k_{x}=\frac{k}{\rho^{(1-a-b-\ldots)}}=\kappa \frac{k_{B} T}{h} K_{x}^{\ddagger}
$$


where $K_{x}^{\ddagger}=\Pi_{i} x_{i}^{\nu_{i}}$. Relating this equilibrium constant to the difference in Gibb's free energy, $\Delta G^{\ddagger}$, between the activated complex and the reactants, we can write the rate constant as

$$
k_{x}=\kappa \frac{k_{B} T}{h} \exp \left(\frac{-\Delta G^{\ddagger}}{R T}\right)
$$

where $R$ is the gas constant. Thus, the rate constant for an elementary reaction step is a function of the difference in Gibb's free energies between the reactants and the transition state. This relationship between kinetics and free energy is the basis for the linear free-energy relationships that are used to correlate kinetics for reaction families.

\section{Pressure effects - Activation volume}

From the transition-state theory rate constant in Eq. 6 and classical thermodynamics, one can show that

$$
\begin{aligned}
\left(\frac{\partial \ln k_{x}}{\partial P}\right)_{T}=\left(\frac{\partial \ln K_{x}^{\ddagger}}{\partial P}\right)_{T}+\left(\frac{\partial \ln \kappa}{\partial P}\right)_{T} & \\
& =-\frac{\Delta v^{\ddagger}}{R T}+\left(\frac{\partial \ln \kappa}{\partial P}\right)_{T}
\end{aligned}
$$

where $\Delta v^{\ddagger}$ is the activation volume, and it is the difference between the partial molar volumes, $\bar{v}$, of the activated complex and the reactants.

$$
\Delta v^{\ddagger}=\bar{v}^{\ddagger}-a \bar{v}_{A}-b \bar{v}_{B}-\cdots .
$$

The second term on the right-hand-side of Eq. 8 is neglected in most developments, either because the transmission coefficient is taken to be equal to unity or because there is insufficient information to evaluate the contribution of this term. Note, however, that experimental measurements of $\left(\partial \ln k_{x} / \partial P\right)_{T}$ will include the effect of pressure on the transmission coefficient. Van Eldik et al. (1989) discuss recent efforts to account for this effect.

Note that the rate constant in Eq. 8 is expressed in pressure-independent units, such as mole fraction. If the rate constant is in pressure-dependent units (e.g., molarity, as in Eq. 4), then the isothermal compressibility $\left(\kappa_{T}\right)$ of the fluid phase will influence the value of the apparent activation volume determined from $(\partial \ln k / \partial P)_{T}$ (Van Eldik et al., 1989; Dooley et al., 1987). The relevant equation is

$$
\begin{aligned}
\left(\frac{\partial \ln k}{\partial P}\right)_{T} & =\left(\frac{\partial \ln k_{x}}{\partial P}\right)_{T}+(1-a-b-\cdots)\left(\frac{\partial \ln \rho}{\partial P}\right)_{T} \\
& =-\frac{\Delta v^{\ddagger}}{R T}+\left(\frac{\partial \ln \kappa}{\partial P}\right)_{T}+\kappa_{T}(1-a-b-\cdots) .
\end{aligned}
$$

The activation volume can be apportioned into two terms (Van Eldik et al., 1989). The intrinsic $\Delta v^{\ddagger}$ accounts for volume changes arising from changes in bond lengths and angles. As such, it contains mechanistic information. The second contribution to $\Delta v^{\ddagger}$ accounts for volume changes arising from electrostriction and other solvent effects. Wu et al. (1991b) have advanced the idea of partitioning the apparent activation volume for a reaction in an SCF into even more parts. They noted that the properties (e.g., dielectric constant, diffusivity) of an SCF near its critical point, unlike the properties of a liquid, can change markedly with pressure and that these changes can also influence kinetics. They proposed that in addition to the intrinsic activation volume, there can exist activation volumes for the effects of pressure on diffusion, electrostatic interactions, the compressibility of the fluid phase, and phase behavior. This suggestion is consistent with Eckert's observation (1972) that a frequent difficulty in interpreting activation-volume data is that they may represent the sum of several different effects, which must be separated for valid interpretation. Brennecke (1993), on the other hand, argued that this approach may miss much of the microscopicscale phenomena that can influence chemical reactions in SCFs.

Activation volumes for reactions in the liquid phase are typically between -50 and $30 \mathrm{~cm}^{3} / \mathrm{mol}$ (Asano and LeNoble, 1978; Van Eldik et al., 1989), whereas apparent activation volumes on the order of $\pm 1000 \mathrm{~cm}^{3} / \mathrm{mol}$ have been reported (e.g., Johnston and Haynes, 1987; Randolph and Carlier, $1992 \mathrm{a}, \mathrm{b})$ for reactions in dilute fluids near their critical points. As one moves away from the critical point, activation volumes approach their liquid-phase values. For example, Johnston and Haynes (1987) reported that the activation volume for decomposition of $\alpha$-chlorobenzyl methyl ether was -6000 $\mathrm{cm}^{3} / \mathrm{mol}$ at $T_{r}=1.04$ and $P_{r} \approx 1.0$, but the activation volume assumed a more liquidlike value of $-71.6 \mathrm{~cm}^{3} / \mathrm{mol}$ at $T_{r}=$ 1.09 and $P_{r}=6.1$. This example also illustrates the general observation that the activation volume is not a constant. Rather, it can vary with both pressure and temperature. Different functional forms have been used to correlate this pressure dependence (Van Eldik et al., 1989).

\section{Reactions in solution (solvent effects)}

Transition-state theory provides a basis for exploring and explaining solvent effects on chemical reactions. Solvent effects are especially important for reactions in SCFs. In some cases one desires to manipulate these effects, whereas in other cases these effects are used to infer molecular-level information. Throughout this section, we will consider only an elementary bimolecular reaction, $A+B \rightarrow C$, but the methods discussed can be readily extended to other types of reactions. From Eq. 4, it is evident that we can relate the rate constant for this bimolecular reaction in a given solvent to the rate constant, $k_{o}$, in an ideal fluid phase as

$$
k:=k_{o}\left(\frac{\gamma_{A} \gamma_{B}}{\gamma_{M^{\ddagger}}}\right)
$$

Eckert (1972) and Eckert et al. (1974) described methods for using molecular thermodynamics to evaluate these activity coefficients and thereby quantify solvent effects on chemical kinetics for some cases. One can use regular solution theory, for example, which gives the activity coefficient as

$$
R T \ln \gamma_{i}=V_{i}\left(\delta_{i}-\bar{\delta}\right)^{2}
$$


where $\delta_{i}$ and $V_{i}$ are the solubility parameter and molar volume of component $i$, and $\bar{\delta}$ is the solubility parameter of the solvent. Combining this activity coefficient model with the rate constant expression in Eq. 11 gives

$$
\ln \left(\frac{k}{k_{o}}\right)=\frac{V_{A}\left(\delta_{A}-\bar{\delta}\right)^{2}+V_{B}\left(\delta_{B}-\bar{\delta}\right)^{2}-V_{M^{\ddagger}}\left(\delta_{M^{\ddagger}}-\bar{\delta}\right)^{2}}{R T} .
$$

This expression allows one to correlate or predict the effect of solvents with different solubility parameters on the rate constant. The data required for the reactants in Eq. 13 are often available, and Eckert et al. (1974) describe how one can use measured molar volumes and these pure-component properties to estimate the required properties for the transition state. Alternatively, one could simplify the problem by assuming that the molar volume of the activated complex is equal to the sum of the molar volumes of the reactants:

$$
V_{M^{\ddagger}}=V_{A}+V_{B}
$$

One then obtains the Neufeld-Herbrandson relation (Moore and Pearson, 1981)

$$
\begin{aligned}
R T \ln \left(\frac{k}{k_{\circ}}\right)=2 \bar{\delta}\left(V_{M^{\ddagger}} \delta_{M^{\ddagger}}\right. & \left.-V_{A} \delta_{A}-V_{B} \delta_{B}\right) \\
& +\left(V_{A} \delta_{A}^{2}+V_{B} \delta_{B}^{2}-V_{M^{\ddagger}} \delta_{M^{\ddagger}}^{2}\right),
\end{aligned}
$$

which shows that the $\log$ of the rate constant ratio should vary linearly with the solvent solubility parameter $\bar{\delta}$. This expectation has been verified experimentally for reactions in a series of different liquid solvents (Eckert et al., 1974; Moore and Pearson, 1981) and for hydrolysis reactions in a single SC solvent (water) wherein the solubility parameter was changed by varying the density (Huppert et al., 1989).

The dielectric constant is another solvent property that has found frequent use in correlating solution-phase reaction kinetics, especially for reactions involving polar molecules and/or transition states. Considering exclusively electrostatics, Kirkwood showed that the free energy of a point dipole with dipole moment $\mu$ and in a cavity of radius $r$ in a medium of dielectric constant $\epsilon$ relative to its free energy in a medium with a dielectric constant of unity is (Connors, 1990)

$$
\Delta G=-\left(\frac{\mu^{2}}{r^{3}}\right) \frac{(\epsilon-1)}{(2 \epsilon+1)}
$$

Combining this expression for the free energy with the transition-state expression for the rate constant leads to

$$
\ln k=\ln k^{\prime}-\left(\frac{N_{a v}}{R T}\right) \frac{(\epsilon-1)}{(2 \epsilon+1)}\left[\frac{\mu_{A}^{2}}{r_{A}^{3}}+\frac{\mu_{B}^{2}}{r_{B}^{3}}-\frac{\mu_{M^{\ddagger}}^{2}}{r_{M^{\ddagger}}^{3}}\right]
$$

where $k^{\prime}$ is the rate constant in a medium with unit dielectric constant and $N_{a v}$ is Avogadro's number. The qualitative insight obtained from Eq. 17 is that the rate constant should increase with the dielectric constant of the medium if the transition state is more polar than the reactants. The quantitative use of Eq. 17 would suggest that the rate constant should vary linearly with the quantity $(\epsilon-1) /(2 \epsilon+1)$ on a semilog plot. This expectation has been confirmed for reactions in the liquid phase (Moore and Pearson, 1981; Connors, 1990). Moore and Pearson (1981) note that the correlating function becomes $(\epsilon-1) / \epsilon$ when one uses a more realistic expression for the free energy of the dipole. Moreover, kinetics are also sometimes correlated simply with $1 / \epsilon$, which is linearly related to $(\epsilon-1) /(2 \epsilon+1)$. Several investigators have correlated rate constants for reactions in SCFs with the solvent dielectric constant (Johnston and Haynes, 1987; Townsend et al., 1988).

Another solvent property that can influence reactions involving ions or polar molecules is the ionic strength $(I)$. To quantify the influence of ionic strength on a chemical reaction involving ions at low concentrations, one can use transition-state theory and the Debye-Hückel expression for the activity coefficients, which applies to dilute solutions. The result is (Moore and Pearson, 1981; Connors, 1990)

$$
\ln k \cong \ln k_{o}+2 Z_{A} Z_{B} \alpha \sqrt{I}
$$

where $Z_{i}$ is the charge on species $i, \alpha$ is a constant, and $k_{o}$ is the rate constant at infinite dilution in a given solvent. One then expects the rate constant to vary with the square root of the ionic strength. Cases such as this, in which the ionic strength influences the rate constant through its influence on the activity coefficients, illustrate a primary salt effect. The medium's ionic strength can also influence reactions between neutral molecules. This influence arises when the transition from reactants to the activated complex involves the development of a charged species or a change in polarity (Moore and Pearson, 1981).

Another means whereby a liquid or SCF can influence a chemical reaction is through cage effects. Cage effects have often been invoked to rationalize the behavior of reactions in SCFs (e.g., Lawson and Klein, 1985; György and Freeman, 1986; Helling and Tester, 1987; Yang and Eckert, 1988), although some recent attempts to identify them unequivocally have been fruitless (O'Shea et al., 1991b; Roberts et al., 1993b). The basic idea underpinning this concept is that solvent molecules can form a "cage" around reactive species. The observed reaction rate will then depend on the rate at which the caged intermediates react with each other and the rate at which they escape from the cage. For example, consider the bond homolysis reaction below:

$$
A-B \stackrel{1}{\leftrightarrow} \underbrace{[A \cdot+B \cdot]}_{\text {solvent cage }} \stackrel{2}{\rightarrow} A \cdot+B \cdot
$$

The production of radicals $A \cdot$ and $B \cdot$ in solution could be diminished by any factor that increases their rate of recombination to form molecule $A-B$ relative to their rate of escape from the solvent cage, presumably via diffusion. Accordingly, high densities, low diffusivities, and high viscosities increase the probability that solvent cage effects may contribute to the observed kinetics. A quantitative accounting for the importance of cage effects requires a knowledge of the kinetics and 
dynamics of the bond-breaking and bond-forming processes and of solvent rearrangement (Liu et al., 1993). Simpler phenomenological models can be developed from the rate constants for step 1 in Eq. 19 and the rate of diffusion for step 2. Similar ideas have been advanced for solvent effects on bimolecular, diffusion-controlled reactions (Moore and Pearson, 1981).

A final means of probing solvent effects on chemical reaction rates that we discuss in this review is the use of solvatochromic shift data (Connors, 1990). Because of solutesolvent interactions, a solute's electronic energy levels can be affected by the solvent. Solvatochromism is the term used to describe a solvent-induced change in the electronic absorption spectrum of a solute. The solvent can influence the wavelength of maximum absorption $\left(\lambda_{\max }\right)$, and this influence is typically reported in terms of a transition energy, $E_{T}$, where

$$
E_{T}=\frac{h c N_{a v}}{\lambda_{\max }}
$$

and $c$ is the speed of light.

Taking phenol blue ( $N, N$-dimethylindoaniline) as a representative solvatrochomic probe, $\lambda_{\max }$ shifts to higher values (lower energies) as the solvent polarity increases. This occurs because the dipole moment of the excited state is 2.5 debye greater than that of the ground state, so the excited state of phenol blue is stabilized more than the ground state as the solvent polarity increases. As a consequence, the transition energy decreases as the polarity of the solvent increases, as is indicated by a red shift in the visible $\pi-\pi^{*}$ absorption band.

Solvatochromic scales are commonly used to correlate solvent effects on rate constants in the liquid state. These measures are also becoming popular in the SCF literature (see the review by Johnston et al., 1989). Rate constants can often be correlated as linear functions of the transition energy as in Eq. 21, where $a$ and $b$ are pressure-independent parameters:

$$
\ln k_{x}=-a E_{T}+b
$$

The activation volume can then be obtained as

$$
\Delta v^{\ddagger}=a R T\left(\frac{\partial E_{T}}{\partial \rho}\right)_{T} \rho \kappa_{T} .
$$

Thus, solvatochromic shift data can be a route to determining activation volumes.

This section gave a brief overview of transition-state theory and reactions in solution. These concepts have been employed to understand reactions in SCFs. One must have a working knowledge of these ideas to appreciate current work on reactions at supercritical conditions.

\section{Fundamentals}

The fundamental chemical and physical phenomena associated with reactions at SC conditions, especially near the critical point, have been a topic of interest for several years. Advances have been made as new experimental results emerge and researchers then attempt to interpret these results and draw generalizations from them. Experimental re- sults are, at times, however, open to divergent interpretations. As an example, consider the interesting chapter in the history of reactions at supercritical conditions that Narayan and Antal (1990) briefly recount. They point out that Krichevskii et al. $(1966,1969)$ reported experimental data that showed dramatic effects on chemical kinetics and chemical equilibrium near the critical point. These investigators reported that the rate of recombination of $\mathrm{Cl}$ atoms was very slow at chlorine densities close to the critical value (Krichevskii et al., 1966) and that the temperature dependence of the equilibrium concentration of $\mathrm{NO}_{2}$ (from $\mathrm{N}_{2} \mathrm{O}_{4}$ decomposition) increased markedly near the critical temperature (Krichevskii et al., 1969). These authors also explained their results using thermodynamic analyses. About fifteen years later, Procaccia and Gitterman (1981, 1982, 1983) offered a different theoretical explanation of these results and concluded that some classes of chemical reactions slow down as the critical point is approached. They also argued that Krichevskii's data for the $\mathrm{N}_{2} \mathrm{O}_{4}=2 \mathrm{NO}_{2}$ equilibrium were a manifestation of the anomalies that appear for chemical equilibria near critical points. This work motivated others (Wheeler and Petschek, 1983; Morrison, 1984; Greer, 1985; Milner and Martin, 1986) to examine Krichevskii's experiments and the thermodynamics of reactions near critical conditions more closely. These articles showed that Krichevskii's data did not provide evidence of anomalies or dramatic effects of chemical reaction rates at near-critical conditions (Morrison, 1984; Greer, 1985), and that the theory developed by Procaccia and Gitterman to explain these purported effects was in error (Wheeler and Petschek, 1983; Milner and Martin, 1986). Wheeler and Petschek (1983), however, did conclude that anomalies do occur in the temperature dependence of chemical equilibria near critical points, but the effects are smaller and more subtle than Procaccia and Gitterman (1981, 1982, 1983) predicted. Likewise, Milner and Martin (1986) showed that a dramatic slowing down of chemical reactions near a critical point is not a reasonable expectation. They concluded that any critical slowing down of a chemical reaction would be difficult to detect experimentally.

More recently, several investigators have applied concepts and experimental tools developed for liquid-phase kinetics to reactions at supercritical conditions to learn about the influence of SCFs on reactions. The concepts in the previous section all flow from transition-state theory, which applies to elementary reaction steps, so one prefers to work with elementary reactions in SCFs to take full advantage of these tools. This realization has prompted a number of investigations with well-characterized reactions in SCFs. At times, these elementary reactions had no apparent technological applications, and the goal of the research was the acquisition of fundamental knowledge. Studies of this type are discussed in this section. Other investigators employed the concepts discussed in the previous section to interpret results from reactions that are, or may become, technologically relevant. We defer the review of these systems until we reach the appropriate applications in the next section.

In addition to having a basic understanding of transitionstate theory and reactions in solution, one must also be aware of the unique properties of and phenomena associated with SCFs near their critical points to understand current work on reactions in SCFs. We noted earlier that the absolute values 
of activation volumes for reactions in dilute SCFs can be very large in the highly compressible region near the critical point. This occurs because the activation volume is the difference of partial molar volumes, the absolute values of which can be very large for a solute at infinite dilution in a fluid near its critical point. Note, however, that very large activation volumes tend to be restricted to dilute solutions very near the critical point of the solvent. The magnitude of the activation volume becomes much smaller as one moves to temperatures or pressures removed from the critical point and as one deals with more concentrated mixtures (Suppes et al., 1989; Chateauneuf et al., 1992; Randolph and Carlier, 1992a,b; Peck et al., 1989; Roberts et al., 1992).

The partial molar volume can be expressed as

$$
\vec{v}_{i}=V_{s} \kappa_{T} n\left(\frac{\partial P}{\partial n_{i}}\right)_{T, V, n_{j \neq i}}
$$

where $V_{s}$ is the molar volume of the solvent, $n$ is the total number of moles in the mixture, and $n_{i}$ is the number of moles of component $i$. The partial molar volume at infinite dilution diverges as one approaches the critical point. The behavior is a manifestation of critical behavior, and it is attributed to long-range, mean-field effects in the near-critical fluid (Economou and Donohue, 1990). For a solvent near its critical point at constant temperature and volume, the pressure decreases with the introduction of a solute because of attractive forces between the solute and solvent molecules. This intermolecular attraction translates into a very large contraction in volume (facilitated by the large isothermal compressibility in the near critical region) in order to balance the pressure. This contraction results in the very large negative partial molar volumes of solutes reported for infinitely dilute SCF solutions (Eckert et al., 1986). This attractive behavior is thought to occur when the solute is larger than the solvent, and the characteristic energy for the solute-solute (and hence solute-solvent) interactions is larger than the solvent-solvent interaction energy. Most supercritical mixtures of practical interest are "attractive" in this sense. We note, however, that molecular dynamics simulations of both attractive and repulsive mixtures have been reported (Chialvo and Debenedetti, 1992).

The attraction of solvent molecules to a solute upon its addition to an SCF disrupts the structure of the SCF solvent and leads to the creation of regions in space near the solute molecule where the local composition and local density differ from their bulk or average values. This phenomenon has been referred to as "clustering," "local density augmentation/enhancement" (e.g., Brennecke et al., 1990a), and "molecular charisma"(Ekart et al., 1993; Eckert and Knutson, 1993). Regardless of the name used to describe the phenomenon, it is important because it can cause the local environment around a solute molecule to differ from the bulk environment. Both experimental (Carlier and Randolph, 1993; Johnston and Haynes, 1987; Kim and Johnston, 1987b; Nakagawa, 1991; Ikushima et al., 1992) and simulation studies (Petsche and Debenedetti, 1989) have pointed to the occurrence of enhanced local density of an SC solvent around solute molecules in dilute solutions. Clustering is generally taken to describe specific, short-range effects (Brennecke et al., 1990a; Carlier and Randolph, 1993), which differ from the long-range correlations due to critical behavior that lead to large partial molar properties in near-critical fluids. O'Brien et al. (1993) have conjectured that local density enhancements were due to "local quasicriticality."

The clustering of solvent molecules about a solute is thought to be a dynamic process. The frequency of exchange of cluster members with molecules from the bulk solvent has been reported to be on the order of a picosecond (Petsche and Debenedetti, 1989), and clusters may persist for $100 \mathrm{pi}$ coseconds (O'Brien et al., 1993). Clustering can affect reaction rates in several ways. The higher local solvent density in a cluster may change local values of density-dependent properties, such as the dielectric constant, and thereby influence reaction rates (Johnston and Haynes, 1987). Moreover, the cluster of solvent molecules may form a "cage" around reactants and/or the transition-state species and thereby alter the progress of some reactions (Ikushima et al., 1991; Combes et al., 1992).

More recently, solute-solute and solute-cosolvent clustering has been observed in SCFs (see Eckert and Knutson, 1993; Kazarian et al., 1993; Tom and Debenedetti, 1993; Chialvo and Debenedetti, 1992; and Brennecke, 1993 and references therein). The presence of solute-solute clusters might be expected to influence the rates of some bimolecular reactions, but they will not influence the rates of diffusion-controlled reactions (Randolph et al., 1994). It should be noted, however, that for solute-solvent clusters or solute-solute clusters to have any influence on a reaction, the time scale of the reaction should be of a similar or smaller order of magnitude than the time scale over which the cluster maintains its integrity (Roberts et al., 1993a). A rough lower limit for the time scale for a chemical reaction can be estimated by noting that the term $\left(k_{B} T / h\right)$ in the transition-state theory rate constant (see Eq. 2) can be thought of as a frequency factor. It is of the order of magnitude of $10^{13} \mathrm{~s}^{-1}$ at $500 \mathrm{~K}$, and this is the same magnitude as the encounter frequency between molecules in a liquid and of slow molecular vibrations (Steinfeld et al., 1989). Thus, the shortest time scale for a chemical reaction would be expected to be about $10^{-14}$ to $10^{-13} \mathrm{~s}$ (10-100 fs).

\section{Kinetics and selectivities of chemical reactions}

Aida and Squires (1987) recognized that SCF media were attractive for chemical reaction studies, and they investigated the photoisomerization of trans-stilbene in $\mathrm{SC} \mathrm{CO}_{2}$, liquid $\mathrm{CO}_{2}$, and $n$-hexane. This isomerization reaction was selected because it had been extensively studied in liquid solvents, and the ratio of cis/trans isomers obtained from the isomerization was known to be a function of the solvent's viscosity. Aida and Squires found that the cis/trans ratio was a strong function of pressure in $\mathrm{SC} \mathrm{CO}_{2}$ and that the existing model of the ratio being controlled by solvent viscosity could accommodate these results in $\mathrm{SC} \mathrm{CO}_{2}$.

Olesik et al. (1986) used a novel capillary flow system and FTIR spectrometry to monitor the decomposition of allyldiisopropylamine oxide in $\mathrm{SC} \mathrm{CO}_{2}$ in situ. Their main objective was to demonstrate the utility of the experimental apparatus. The only reaction observed in conventional liquid solvents, such as deuterated chloroform, and in carbon dioxide at 
moderate pressure $\left(68 \mathrm{~atm}, 50^{\circ} \mathrm{C}\right.$ ), was a rearrangement to $O$-allyl- $N, N$-diisopropylhydroxylamine. When the reaction was conducted in carbon dioxide at higher pressures (98.67 and $115.7 \mathrm{~atm}$ ), however, the $O$-allyl- $N, N$-diisopropylhydroxylamine decomposed further to acrolein. Moreover, the reaction rate constant decreased modestly as the pressure increased from 98.67 atm to 115.7 atm. The authors suggested that the rearrangement was the rate-limiting step in the high-pressure reactions, so that the rate constants reported were for this step and not for the elimination reaction.

Sigman and Leffler (1987) and Sigman et al. (1987) have also conducted reactions in $\mathrm{SC} \mathrm{CO}_{2}$. They used $\mathrm{SC} \mathrm{CO}_{2}$ in their studies because it enabled them to adjust the properties of the solvent without changing its structure. By comparing and integrating results in $\mathrm{SC} \mathrm{CO}_{2}$ with results in other (liquid) solvents, Sigman and Leffler were able to resolve mechanistic issues. For example, one reaction they studied was the isomerization of 4-(diethylamino)-4'-nitroazobenzene in $\mathrm{SC} \mathrm{CO}_{2}$ at $32.4^{\circ} \mathrm{C}$. There existed some controversy in the literature about the precise mechanism of this isomerization. Some investigators claimed that all azobenzene isomerizations proceed by inversion, but others believed that some isomerizations are inversions and that others are rotations. The properties of the solvent used for the isomerization would be expected to affect these two mechanisms differently. Based on their results, and those in the literature for isomerizations in different solvents, Sigman and Leffler (1987) concluded that the isomerization could proceed through either of the two postulated mechanisms. The dominant reaction mechanism is inversion in $\mathrm{SC} \mathrm{CO}_{2}$ and in alkanes but the mechanism is rotation in more polar solvents.

Troe and coworkers (Otto et al., 1984; Troe, 1986; Gehrke et al., 1990) and Yoshimura and Kimura (1991) have examined the kinetics and dynamics of simple reactions in fluids over very wide density ranges. Their work was motivated in part by a desire to better understand reaction dynamics in condensed phases and in part to develop a comprehensive model for kinetics and dynamics that accounted for the reaction environment, and more specifically, reactant-solvent interactions. Much of Troe's work dealt with reactions in compressed permanent gases (such as Ar and He) and in liquids (such as heptane), but some focused specifically on reactions in SCFs (such as $\mathrm{CO}_{2}, \mathrm{C}_{2} \mathrm{H}_{6}$, and $\mathrm{SF}_{6}$ ). Figure 6, which gives the quantum yields for iodine photolysis in $\mathrm{CO}_{2}$ as a function of the reciprocal diffusivity and density, displays representative results. Troe and his colleagues formulated models that accounted for the influence of diffusion (cage effects) on the observed reaction rates and that invoked the formation of van der Waals clusters to explain their experimental observations. Yoshimura and Kimura (1991) studied the kinetics of the dissociation of 2-methyl-2-nitrosopropane in $\mathrm{SC} \mathrm{CO}_{2}$ at $35^{\circ} \mathrm{C}$ over a wide range of densities. They reported that the dissociation rate constant decreased as the density increased, but the density dependence was smallest in the medium-density region. The activation volume in the low-density region was reported as $300 \mathrm{~cm}^{3} / \mathrm{mol}$. Kimura and Yoshimura reported more extensively on the effects of density on chemical equilibrium for this reaction, and these articles are reviewed in the following subsection.

Fluorescence spectroscopy has been used to investigate reactions involving species in excited states in SCFs. Okada et

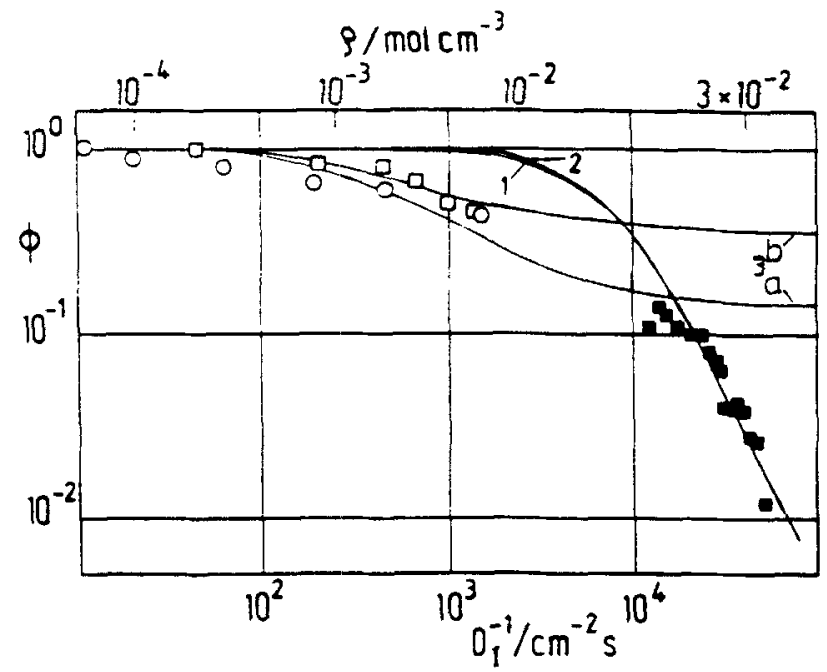

Figure 6. Quantum yields for $I_{2}$ photolysis in $\mathrm{CO}_{2}$ as a function of reciprocal diffusivity and density.

Open symbols are gas-phase results, and filled symbols are liquid-phase results. Curves are calculations from different models (Otto et al., 1984).

al. (1986), for example, briefly report on the intramolecular exciplex formation of $p$-( $N, N$-dimethylaminophenyl)$\left(\mathrm{CH}_{2}\right)_{2}(9$-anthryl) in SC ethylene and trifluoromethane. They used an SCF because it allowed them to change the solvent properties without changing the solvent. These investigators concluded that fluorescence spectroscopy of excited-state species in SCFs gives information about the local solute environment and about solute-solvent interactions.

More recently, Brennecke and Eckert (1989b) and Brennecke et al. (1990b) probed the formation of naphthalene/triethylamine exciplexes and pyrene excimers in SCFs. They reported a strong effect of pressure on the steady-state formation of naphthalene-triethylamine exciplexes in $\mathrm{SC} \mathrm{CO}_{2}$. The authors hypothesized that the reaction that formed the exciplex from excited naphthalene and ground-state triethylamine would be the most pressure sensitive in the reaction network. They then used the Peng-Robinson equation of state and accounted for the effect of electrostriction on the partial molar volumes for the species involved in the exciplex formation reaction to predict the effect of pressure on this reaction. The predictions were qualitatively consistent with the experimental observations. Additionally, Brennecke et al. (1990b) reported the formation of pyrene excimers (an association of a ground-state and an excited-state pyrene molecule), even at very low pyrene concentrations, in SCFs. To obtain an equivalent level of excimer formation in a liquid solvent would have required a 40 -fold higher pyrene concentration. The authors recognized that the excimer formation reaction should be diffusion controlled and that the diffusivity in $\mathrm{SC} \mathrm{CO}_{2}$ exceeded the diffusivity in liquid solvents, but they argued that the diffusivity difference alone could not account for the full magnitude of the observed effect. Brennecke et al. (1990b) invoked enhanced solute-solute interactions in the SCF to explain the apparently enhanced pyrene excimer formation in SCFs.

Zagrobelny et al. (1992) and Zagrobelny and Bright (1992b, 1993) reported the results of a more detailed study of pyrene 
excimer formation in SCFs. They used time-resolved fluorescence along with steady-state measurements to study the kinetics more fully. They sought, but found no evidence for, ground-state solute-solute associations in $\mathrm{SC} \mathrm{CO}_{2}, \mathrm{C}_{2} \mathrm{H}_{4}$, and $\mathrm{CF}_{3} \mathrm{H}$, or in $\mathrm{SC} \mathrm{CO}_{2}$ with acetonitrile and methanol as entrainers. Moreover, they measured the rate constant for excimer formation and found that the influence of density in $\mathrm{SC} \mathrm{CO}_{2}$ and $\mathrm{C}_{2} \mathrm{H}_{4}$ was entirely consistent with that expected for a diffusion-controlled bimolecular reaction. Excimer formation in $\mathrm{SC} \mathrm{CF}_{3} \mathrm{H}$ and in $\mathrm{CO}_{2} / \mathrm{MeOH}$ and $\mathrm{CO}_{2} / \mathrm{MeCN}$ mixtures, however, proceeded more slowly than the rate predicted for a diffusion-controlled reaction. The authors suggested that $\mathrm{CF}_{3} \mathrm{H}$ and entrainer $/ \mathrm{CO}_{2}$ clusters shield the excited-state pyrene molecules, thereby impeding excimer formation. The authors concluded that pyrene excimers were observed in $\mathrm{SC} \mathrm{CO}_{2}$ and $\mathrm{C}_{2} \mathrm{H}_{4}$ at pyrene concentrations well below those required in a liquid solvent because the SCF had a lower viscosity (higher diffusivity) and SCF-excimer interactions stabilized the excimer excited state. There was no ground-state solute-solute clustering.

Johnston's group at the University of Texas has published several fundamental studies of reactions in SCFs. Kim and Johnston $(1987 \mathrm{a}, \mathrm{b})$ obtained solvatochromic data for phenol blue in $\mathrm{SC}$ ethylene and $\mathrm{SC} \mathrm{CO}_{2}$. They then used these measured $E_{T}$ values with kinetics correlations in the literature in the form of Eq. 21 to predict the rate constants for the Menschutkin reaction of tripropylamine and methyl iodide and the Diels-Alder cycloaddition of isoprene and maleic anhydride as a function of pressure. For both reactions, the rate constants $\left(k_{x}\right)$ were predicted to be strong functions of pressure near $P_{c}$, but the pressure dependence became less remarkable at pressures much greater than $P_{c}$. The activation volume for the Menschutkin reaction, for example, was $-5000 \mathrm{~cm}^{3} / \mathrm{mol}$ at $66 \mathrm{bar}$, and it was $-80 \mathrm{~cm}^{3} / \mathrm{mol}$ at 350 bar. For comparison, the activation volume in liquid $\mathrm{CCl}_{4}$ is $-65 \mathrm{~cm}^{3} / \mathrm{mol}$.

Kim and Johnston also reported that one could use transition energy data to determine the local density of an SCF solvent in the vicinity of a solute. Treating the solvent as a homogeneous polarizable dielectric, one may calculate the intrinsic solvent strength, $E_{T^{0}}$, from Eq. 24 (McRae, 1957)

$$
E_{T^{0}}=A\left(\frac{n^{2}-1}{2 n^{2}+1}\right)+B\left(\frac{\epsilon-1}{\epsilon+2}-\frac{n^{2}-1}{n^{2}+2}\right)+C
$$

where $n$ is the refractive index and $A, B$, and $C$ are parameters that depend on the properties of the solute. Values of $E_{T^{0}}$ from Eq. 24 agreed with measured $E_{T}$ values at high solvent densities, but they were generally higher than experimental $E_{T}$ values in the compressible region because Eq. 24 neglects the additional solvation from clustering that is due to attractive forces (Johnston and Haynes, 1987). This difference between $E_{T}$ and $E_{T^{0}}$ was considered to reflect the difference between the local solvent density about a solute, $\rho_{12}^{l}$, and the bulk density, $\rho$. Kim and Johnston (1987a, b) then advanced statistical mechanical arguments to show that the local density enhancement should be a linear function of the isothermal compressibility of the solvent

$$
\frac{\rho_{12}^{l}-\rho}{\rho}=a^{\prime} \kappa_{T}+b^{\prime}
$$

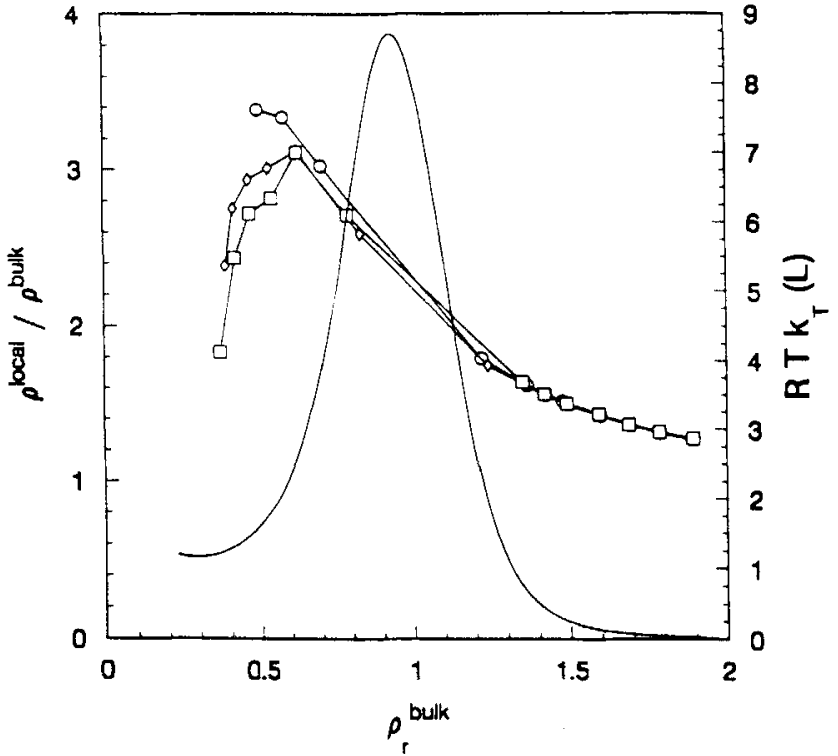

Figure 7. Local density enhancement about ditertbutyl nitroxide radicals (discrete points correspond to different concentrations) and isothermal compressibility (smooth curve) as a function of reduced bulk density for SC ethane at 308 K (Carlier and Randolph, 1993).

where $a^{\prime}$ and $b^{\prime}$ are functions of temperature. Debenedetti (1987) derived a similar result independently. We note, however, that Carlier and Randolph (1993) and O'Brien et al. (1993) have demonstrated that the local density enhancement does not generally correlate with the isothermal compressibility. Figure 7 gives a representative result. Carlier and Randolph (1993) and O'Brien et al. (1993) argue that local density enhancements are short-range effects. These enhancements are not directly related to the long-range fluctuations that give rise to the maximum in isothermal compressibility near the critical point.

Johnston and Haynes (1987) reported data for the unimolecular decomposition of $\alpha$-chlorobenzyl methyl ether in SC 1,1-difluoroethane that verified the earlier prediction of the existence of extremely large solvent effects on reaction rate constants. They measured rate constants at 403,423 , and $453 \mathrm{~K}$ in a pressure range of 44.8 to 344.7 bar. The rate constant was relatively insensitive to pressure well above the critical point $(P>130 \mathrm{bar})$, but at $403 \mathrm{~K}$ the rate constant increased by an order of magnitude as the pressure increased modestly from 44.8 bar to $60 \mathrm{bar}$ (see Figure 2). The activation volume was reported as $-6,000 \mathrm{~cm}^{3} / \mathrm{mol}$ near the point of maximum compressibility, but the activation volume assumed a more liquidlike value of only $-72 \mathrm{~cm}^{3} / \mathrm{mol}$ at $P_{r}=$ 6.1. Solvatochromic data were also obtained for phenol blue in SC difluoroethane. These data were then used to correlate the rate constant in the form of Eq. 21 and to calculate the local density enhancement. The values of the local density were then used to calculate the local dielectric constant experienced by a solute. This local dielectric constant was much less pressure sensitive than the bulk dielectric constant, and it gave a very good correlation of the kinetics on a Kirkwood plot. 
Hrnjez et al. (1989) showed that SCFs can be used to shift stereoselectivity and regioselectivity in chemical reactions. They found that these selectivities for the photodimerization of isophorone in $\mathrm{SC} \mathrm{CHF}_{3}$ and $\mathrm{SC} \mathrm{CO}$ were functions of pressure. Figure 4 gives some results from this study. The authors attributed the selectivity shifts to pressure-induced changes in the solvent polarity and reorganization.

In subsequent work, O'Shea et al. (1991b) and Combes et al. (1992) examined cage effects in SCFs. O'Shea et al. used the photofragmentation of dibenzylketones and the subsequent combination of benzyl radicals to look for cage effects in $\mathrm{SC}$ ethane and $\mathrm{CO}_{2}$. This reaction exhibits no cage effects in liquids, but does exhibit significant cage effects in organized assemblies such as micelles. These investigators sought to determine whether SCF solvent clusters about a solute formed cages more rigid than those encountered in liquid media. Their experiments gave a statistical distribution of recombination products, which demonstrated the absence of cage effects. Combes et al. (1992), on the other hand, reexamined literature results for the decomposition of $I_{2}$ in ethane (Otto et al., 1984) over a very wide range of densities and found that a model that included cage effects arising from solvent-solute clustering gave a nearly quantitative fit of the experimental data. An otherwise identical model that omitted clustering resulted in good representation of the data in the gas and liquid phases, but a poorer representation in the near-critical region.

Combes et al. (1992) also probed solute-solute clustering in SCFs by examining the photodimerization of 2-cyclohexene1-one in SC ethane. In this reaction, cyclohexenone absorbs a photon and is excited to the singlet state, whereupon it undergoes rapid intersystem crossing to the triplet state. This triplet state can either be deactivated by the solvent or it can react with a ground-state cyclohexenone to form a dimer. This reaction was selected because it is bimolecular and there is a large effect of the solvent polarity on the regioselectivity [head-to-head (HH) vs. head-to-tail (HT)] of the photodimers. The more polar $\mathrm{HH}$ isomer is favored in more polar media. Combes et al. (1992) observed that the quantum yield of the dimerization increased abruptly near $P_{c}$ as the pressure decreased. This could have been the result of less deactivation of the triplet because of fewer collisions with the solvent, or the result of more frequent encounters between the triplet and the ground state facilitated by solute-solute clustering. The regioselectivity also increased abruptly near $P_{c}$ as the pressure decreased. Since it was unlikely that the deactivation of the HT isomer by the solvent would be any more frequent than the deactivation of the $\mathrm{HH}$ isomer, solute-solute clustering (ground-state cyclohexenone molecules around the triplet) was suggested to be the reason for the increase in regioselectivity. These researchers verified that higher solute concentrations did indeed increase the regioselectivity by varying the cyclohexenone concentrations at constant pressure and temperature.

The group at Notre Dame has also been using chemical reactions to explore solvent effects and intermolecular interactions in SCFs. Chateauneuf et al. (1992) and Roberts et al. (1992) used laser flash photolysis to study hydrogen atom abstraction from 2-propanol and 1,4-cyclohexadiene (a nonhydrogen bonding substrate) by triplet benzophenone in $\mathrm{SC} \mathrm{CO}_{2}$ at temperatures of 33 and $44.4^{\circ} \mathrm{C}$ in a

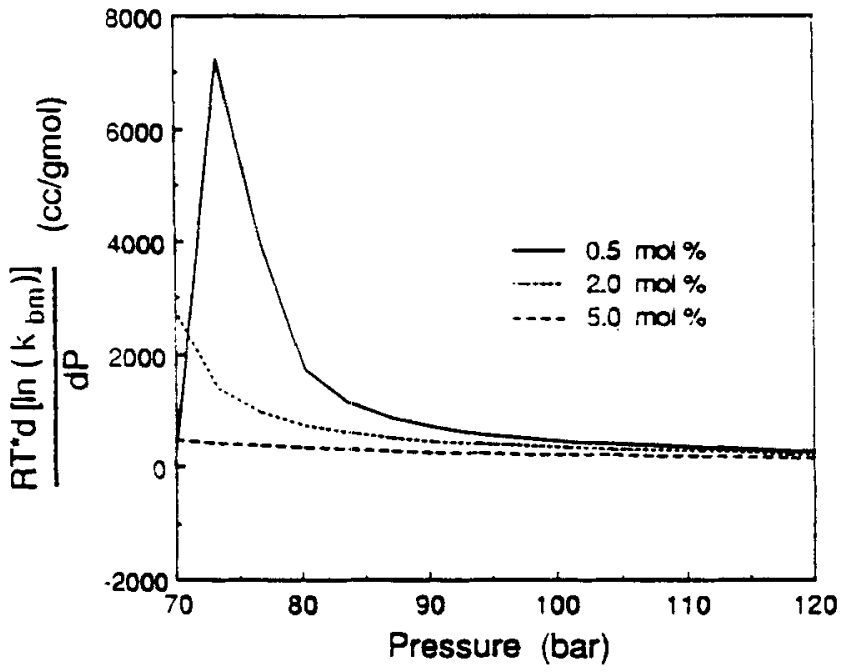

Figure 8. Effect of pressure predicted for the rate constant for benzophenone triplet plus isopropanol in $\mathrm{SC} \mathrm{CO}_{2}$ at different alcohol concentrations (Chateauneuf et al., 1992).

Courtesy of American Chemical Society.

pressure range of 71 to 135 bar. They observed that the bimolecular rate constant at $33^{\circ} \mathrm{C}$ increased sharply as the pressure decreased near the critical pressure. At $44.4^{\circ} \mathrm{C}$, this increase in the rate constant was more modest. This pressure effect, where the rate constant decreased with increasing pressure, was the opposite of the thermodynamic pressure effect predicted by transition-state theory, as discussed earlier. The Peng-Robinson equation of state was used to estimate the partial molar volumes and hence the activation volume for the reaction, and Figure 8 gives representative results of these calculations. The authors argued that cage effects could not be responsible for their results because the time constant for the reaction was longer than the lifetime of a solvent cluster or cage. The authors ventured that the observed behavior was due to the local concentration of solute (benzophenone) and cosolvent (such as 1,4-cyclohexadiene) exceeding their bulk concentrations in the highly compressible near-critical region. These concentration enhancements in the near-critical region were larger than the influence of the activation volume on the rate constant, so the apparent bimolecular rate constant increased with decreasing pressure as one approached the critical point. This scenario was consistent with earlier work (Brennecke et al., 1990b), which showed that solute-solute interactions can be important even in dilute SCF solutions.

Ellington et al. (1994) and Ellington and Brennecke (1993) reported that the apparent bimolecular rate constant for the esterification of phthalic anhydride with methanol in $\mathrm{SC} \mathrm{CO}_{2}$ at 40 and $50^{\circ} \mathrm{C}$ increased with decreasing pressure as one approached the critical point. The 30 -fold increase in the rate constant at $50^{\circ} \mathrm{C}$ greatly exceeded that which the authors expected from the estimated activation volume for the reaction. Thus, the authors attributed the enhanced reaction rate constant to the concentration of methanol about the phthalic anhydride molecules exceeding the bulk, average methanol concentration. The authors used estimated local densities and local compositions to show that this explanation was reason- 


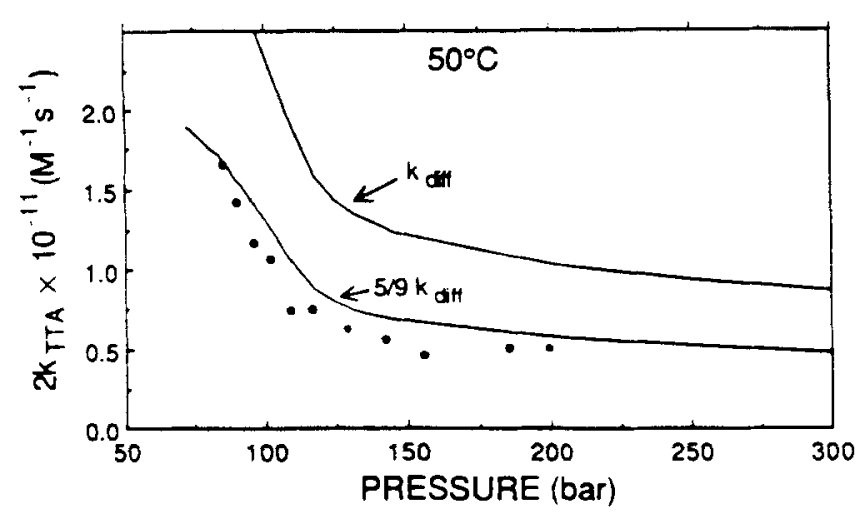

Figure 9. Rate constant for diffusion-controlled bimolecular reaction (triplet-triplet annihilation of benzophenone) in $\mathrm{SC} \mathrm{CO}_{2}$ at $50^{\circ} \mathrm{C}$.

The $5 / 9 k_{\text {diff }}$ accounts for the spin statistical factor (Roberts ct al., 1993a).

Courtesy of American Chemical Society.

able. Significantly, this work reported reaction rate enhancements at a temperature $\left(50^{\circ} \mathrm{C}\right)$ and pressure $(97.5 \mathrm{bar})$ that were well above the estimated critical values for the mixture.

In subsequent work probing the influence of clustering on reactions in SCFs, Roberts et al. (1993a, b) used laser flash photolysis to examine the triplet-triplet annihilation (TTA) process of benzophenone and the self-termination reaction of the benzyl radical in $\mathrm{SC} \mathrm{CO}_{2}$, ethane, $\mathrm{CHF}_{3}$, and $\mathrm{CO}_{2} / \mathrm{CH}_{3} \mathrm{CN}$ mixtures. These two processes proceed through diffusion-controlled bimolecular reactions in the liquid phase. The bimolecular rate constants measured experimentally and adjusted by their spin statistical factors indicated that the reactions also occurred at the diffusion limit in the different SCFs. A comparison of the experimental rate constants and those expected for a diffusion-controlled reaction in $\mathrm{SC} \mathrm{CO}_{2}$ is given in Figure 9. The authors found no evidence for altered reactivity due to supercritical solvent clustering about the diffusive encounter pairs or due to solute-solute clustering. This absence of an SCF solvent effect was explained in terms of the lifetime of the dynamic solvent clusters being much shorter than the characteristic time for the process being studied. These authors also noted the absence of any measurable effects attributable to solute-solute clustering.

Randolph and Carlier (1992a,b) used electron paramagnetic resonance spectroscopy to study a Heisenberg spin-exchange reaction of di-tert-butylnitroxide free radicals in SC ethane. In this reaction, the free radicals with unpaired electrons of antiparallel spins exchange spin states upon collision. Since the products and reactants are chemically identical, their concentrations can be accurately specified. Moreover, the local polarity affects the nitrogen hyperfine coupling constant, $A_{N}$, by increasing the spin density on nitrogen. The shift in $A_{N}$ is a function of the local dielectric constant (McRae, 1957), which in turn depends on the local density. Thus, this system can be used to probe the local density in the cybotactic region surrounding the solute molecule. The ratio of the local density to the bulk density was reported to exceed 4.0 at $58^{\circ} \mathrm{C}$ and pressures around $6 \mathrm{MPa}$. At $35^{\circ} \mathrm{C}$ the reaction rate constant decreased sharply with increasing pressure, particularly in the near critical region. This trend was also evident but less pronounced at higher temperatures further away from $T_{c}$. This variation of the rate constant with pressure led to large positive activation volumes being measured for this reaction $\left(7,500 \mathrm{~cm}^{3} / \mathrm{mol}\right.$ near $P_{c}$ at $35^{\circ} \mathrm{C}$, see Figure 10). The authors used the Stokes-Einstein hydrodynamic theory and the Benedict-Webb-Rubin equation of state to predict the activation volume, which for this diffusion-controlled
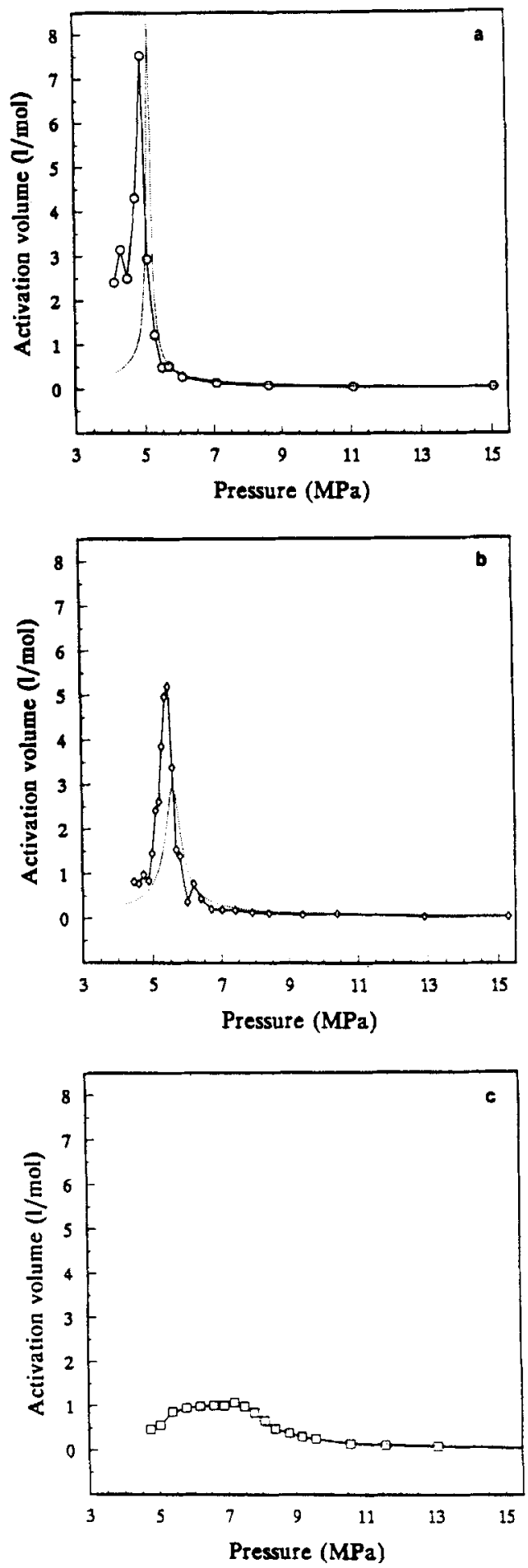

Figure 10. Activation volumes for diffusion-controlled reaction in SC ethane at 308,313 , and $331 \mathrm{~K}$ (Randolph and Carlier, 1992a).

Courtesy of American Chemical Society. 
reaction largely reflects collision frequencies. The predicted values of $\Delta v^{\ddagger}$ and their dependence on pressure and temperature agreed with experimental results. Thus, solute-solvent clustering appeared to have no influence on the collision frequency. Further analysis showed, however, that the experimental collision times (duration of a collision) were as much as three times longer than those predicted from the StokesEinstein theory. This ratio of experimental to predicted collision times was greatest near the critical point. It decreased to unity at higher pressures. Solvent-solute clustering was thought to be responsible for this phenomenon. That clustering affected the collision time but not the collision frequency, which is related to translational diffusion, was consistent with the lifetime of solvent clusters being short in comparison to diffusional time scales, but long in comparison to the duration of the solute-solute collision.

Randolph et al. (1994) and Ganapathy et al. (1993) subsequently employed molecular dynamics and Brownian dynamics calculations to examine the influence of clustering on collision and reaction rates for rapid bimolecular reactions in SCFs as a function of density. They pointed to the apparent ambiguity in the literature, wherein solvent-solute clusters affected some reactions (Zagrobelny et al., 1992; Zagrobelny and Bright, 1992b; Randolph and Carlier, 1992a,b; Combes et al., 1992; Ikushima et al., 1992) but not others (Roberts et al., 1993a,b; Combes et al., 1992) and solute-solute clusters were important in some systems (Combes et al., 1992; Gupta et al., 1993) but not in others (Roberts et al., 1993), as motivation for their work. These investigators found that, consistent with expectations, the reaction rate constants approached the hard-sphere collision theory limit at low densities and the Smoluchowski limit for diffusion-controlled reactions at high densities. Their calculations revealed that the effects of solute-solute clusters in SCFs on reaction rates for diffusion-controlled bimolecular reactions may not be apparent because the concentration gradient imposed by the diffusion limitation mutes the influence of the strong solute-solute correlations. This effect is most important at high densities where diffusion limitations set in. They noted, however, that solute-solute correlations always influenced the molecular collision rates, but they influenced reaction rates only for certain combinations of the collision diameter and density.

Balbuena et al. (1994) also conducted a molecular dynam. ics study of a reaction in an SCF. They reported the potential of mean force along the reaction coordinate for the $S_{N^{2}}$ reaction $\mathrm{Cl}^{-}+\mathrm{CH}_{3} \mathrm{Cl}=\mathrm{ClCH}_{3}+\mathrm{Cl}^{-}$in $\mathrm{SC}$ water. They found that the energy profile for this reaction in $\mathrm{SC}$ water was similar to the profile in ambient water. The authors concluded that the molecular environment in SC water is more like ambient water than it is like a polar organic solvent (e.g., dimethylformamide).

\section{Reaction equilibria}

The previous section reviewed studies that used chemical kinetics to probe intermolecular interactions in SCFs. This section discusses studies that used chemical equilibrium for this purpose. The solvent influences reaction thermodynamics through the environment it produces around the solutes. Among the important solvent properties characterizing this reaction environment are the polarity and the polarizability.

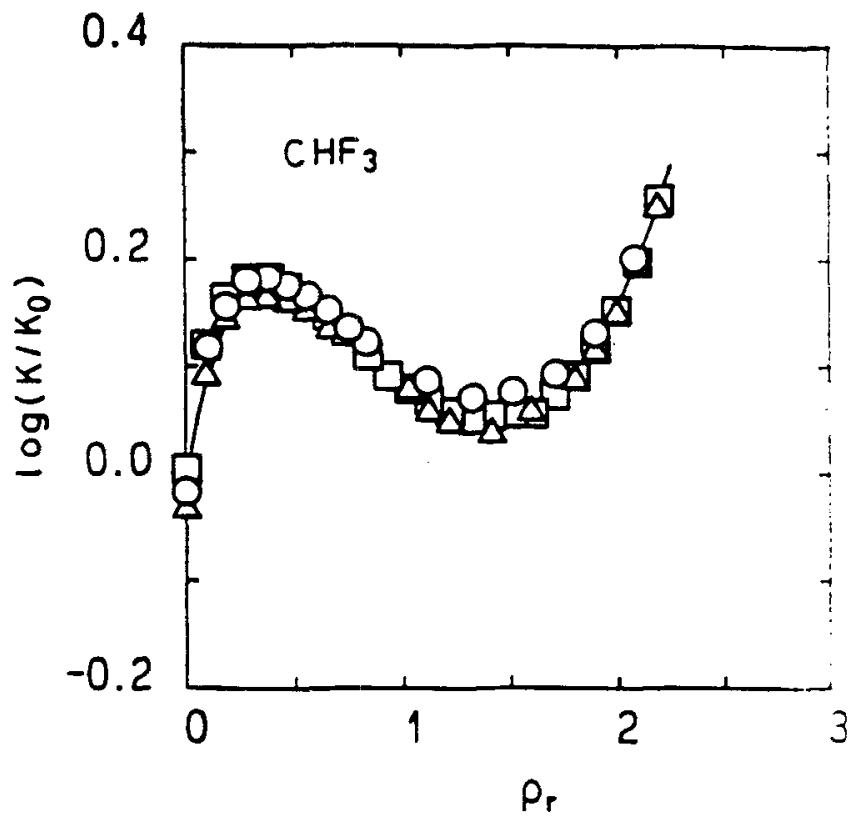

Figure 11. Equilibrium constant for 2-methyl-2-nitrosopropane dimerization in $\mathrm{CHF}_{3}$ at $60.9^{\circ} \mathrm{C}$ as a function of reduced density (Kimura and Yoshimura, 1992a).

The solvent polarity corresponds to the permanent dipole-dipole interactions. The polarizability, on the other hand, is indicative of induction forces, where the solute molecule induces a dipole in the solvent. Thus, measuring equilibria in SCFs can give insight into molecular interactions.

Kimura et al. (1989) and Kimura and Yoshimura (1992a,b) addressed the problem of how fluid structure influences chemical reactions by examining the effect of solvent density on the equilibrium constant for the dimerization of 2-methyl2-nitrosopropane in $\mathrm{CO}_{2}, \mathrm{CClF}_{3}, \mathrm{CHF}_{3}, \mathrm{Ar}$, and $\mathrm{Xe}$. Their studies were typically at supercritical temperatures, and the densities investigated ranged from gas-phase conditions to about 2.5 times the critical density. Their experimental results showed that the equilibrium constant in $\mathrm{CO}_{2}, \mathrm{CCIF}_{3}$, and $\mathrm{CHF}_{3}$ increased with density in the low-density region $\left(\rho_{r}<0.3\right)$, then decreased with density in the medium-density region $\left(0.3<\rho_{r}<1.4\right)$, and then increased with density once more in the high-density region $\left(\rho_{r}>1.4\right)$. Figure 11 provides representative results. The authors argued that these three regions appeared because the dominant molecular interactions within the fluid were different within each of these regions. In the low-density region, attractive intermolecular forces and solute-solvent interactions determined the effect of density. Accordingly, behavior in this low-density region varied with the identity of the solvent and with temperature. In the high-density region, a "packing effect" dominates. In the medium-density region, multibody interactions played a major role, and solvent-solvent interactions became very important. Comparing their results in Xe with those in the molecular fluids, the authors concluded that the density dependence of the equilibrium constant is not affected much by the details of the molecular geometry of the solvent in the medium- and high-density regions. 
There have been several studies of tautomeric equilibria in SCFs aimed at uncovering fundamental molecular information. Peck et al. (1989) used in situ UV spectroscopy to determine equilibrium constants, $K_{c}$, for the tautomeric equilibria of 2-hydroxypyridine and 2-pyridone in SC propane at $393 \mathrm{~K}$ $\left(T_{r}=1.06\right)$ and 1,1-difluoroethane at $403 \mathrm{~K}\left(T_{r}=1.04\right)$. The equilibrium constants increased with pressure in both fluids. Figure 5 gives selected results from this study. In difluoroethane, for instance, the equilibrium constant increased from 0.82 at 20.68 bar to 4.64 at 103.4 bar and finally to 5.77 at 206.8 bar. Clearly, the effect of pressure diminished as the pressure was increased well beyond $P_{c}$ (45 bar).

The tautomers 2-hydroxypyridine and 2-pyridone have similar sizes so the effect of pressure on their equilibrium reflects the difference in their polarities. The relationship between $K_{c}$ and solvent polarity can be described, at least qualitatively, from Kirkwood (1934) theory as

$$
R T \ln K_{c}=\frac{3}{8}\left(\frac{1-\epsilon}{1+\epsilon}\right)\left(\frac{\mu_{2}^{2}}{r_{2}^{3}}-\frac{\mu_{3}^{2}}{r_{3}^{3}}\right)
$$

where $r$ is the molecular radius, and the subscripts 2 and 3 refer to the two tautomers. Figure 12 displays the correlation of the equilibrium constant with the dielectric constant. As the solvent polarity increases, the equilibrium shifts toward 2-pyridone, the more polar tautomer. The data revealed that $K_{c}$ reached higher values in the polar solvent 1,1-difluoroethane than in nonpolar propane because the solvent dielectric constant of the former is larger for a given range of reduced pressure. On the other hand, at a given value of the dielectric constant, $K_{c}$ was larger in propane than in difluoroethane, presumably because propane has a larger polarizability per unit volume. Peck et al. also showed that the equilibrium constant could be correlated with solvatochromic shift data for phenol blue.

The effect of pressure on $K_{c}$ became less pronounced at high pressures where the density became a much weaker function of pressure and the isothermal compressibility $\left(\kappa_{T}\right)$ was much smaller. This behavior is entirely consistent with the effect of pressure on the equilibrium constant (Hamman, 1963), which depends on $\Delta \bar{v}_{r x h}$ and $\kappa_{T}$ as given in Eq. 27:

$$
\left(\frac{\partial \ln K_{c}}{\partial P}\right)_{T}=\frac{-\Delta \bar{v}_{r x n}}{R T}+\kappa_{T} \Sigma \nu_{i}
$$

Note the analogy with Eq. 10, which gives the effect of pressure on a rate constant. Equation 27 includes $\Delta \bar{v}_{r x n}$ rather than the activation volume in Eq. $10 ; \Delta \bar{v}_{r x n}$ is defined as the difference between the partial molar volumes of the products and the reactants. For a tautomeric equilibrium, $\Sigma \nu_{i}$, the sum of the stoichiometric coefficients, is zero. The isothermal compressibility still influences the pressure dependence of $K_{c}$, however, because it affects the partial molar volumes of the product and reactant, which contribute to $\Delta \bar{v}_{r x n}$ (recall Eq. 23). Since the partial molar volume at infinite dilution diverges as one approaches the critical point, one would expect the effect of pressure on $K_{c}$ to be greatest near the critical point. Peck et al. used Eq. 27 to calculate $\Delta \bar{v}_{r x n}$ as a function of pressure, and $\Delta \bar{v}_{r x n}$ was most negative near the critical pressure, as shown in Figure 13.

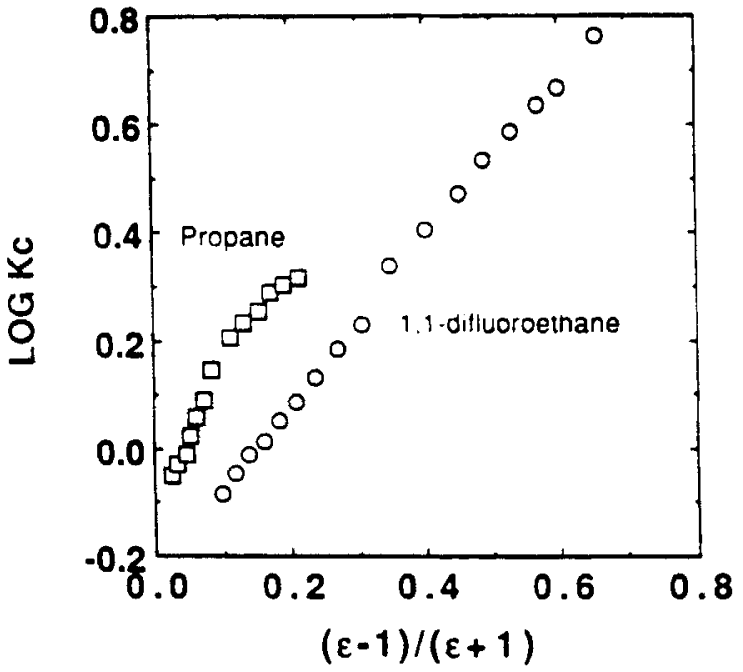

Figure 12. Kirkwood plot for equilibrium constant for tautomerization of 2-hydroxypyridine/ 2-pyridone in SC propane at $393 \mathrm{~K}$ and SC 1,1-difluoroethane at $403 \mathrm{~K}$ (Peck et al., 1989).

Courtesy of American Chemical Society.

Although $K_{c}$ was a highly nonlinear function of pressure, $\ln K_{c}$ was nearly linear in density. The dependence of $K_{c}$ on density is related to the pressure dependence by Eq. 28, where we also used Eq. 23 and the definition of $\Delta \bar{v}_{r x n}$ to obtain the final equality.

$$
\begin{aligned}
R T\left(\frac{\partial \ln K_{c}}{\partial \rho}\right)_{T} & =\frac{R T}{\rho \kappa_{T}}\left(\frac{\partial \ln K_{c}}{\partial P}\right)_{T} \\
= & \frac{-n}{\rho^{2}}\left[\left(\frac{\partial P}{\partial n_{3}}\right)_{T, V, n_{1}, n_{2}}-\left(\frac{\partial P}{\partial n_{2}}\right)_{T, V, n_{1}, n_{3}}\right]
\end{aligned}
$$

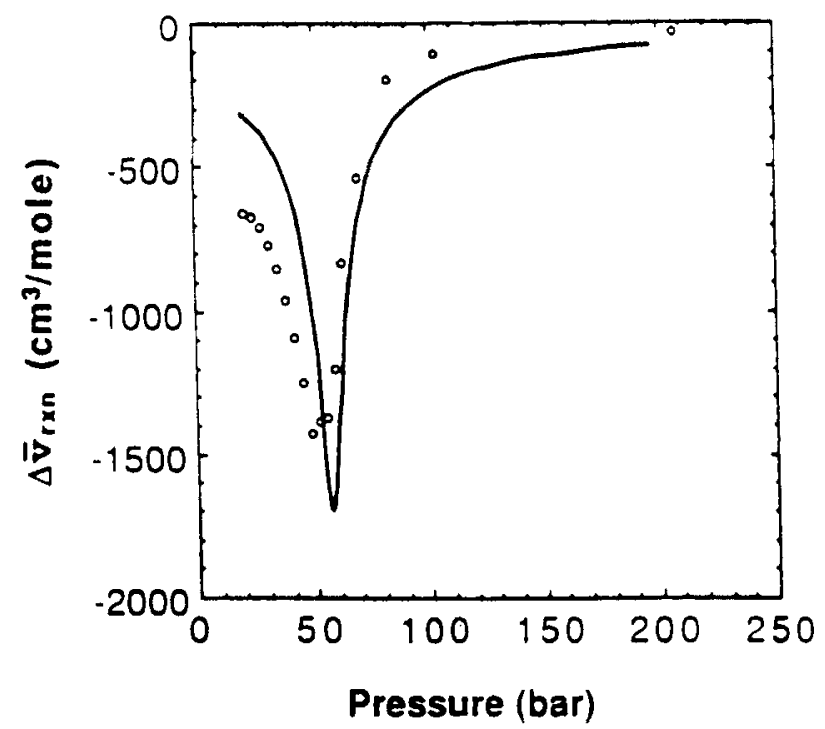

Figure 13. $\Delta \bar{v}_{r x n}$ for tautomerization of 2-hydroxypyridine/2-pyridone in SC 1,1-difluoroethane at 403 K (Peck et al., 1989).

Courtesy of American Chemical Society. 
Equation 28 shows that the effect of density on $K_{c}$ is less complex near the critical point than is the effect of pressure because $\kappa_{T}$ has been eliminated. The authors observed that $\ln K_{c}$ was nearly linear in density. Recall, however, that this behavior need not generally occur (see Kimura and Yoshimura, 1992a, b). The authors used this nearly linear relation between $\ln K_{c}$ and density and a theoretical development based on Eq. 28 and the van der Waals equation of state to determine the difference between the solute-solvent van der Waals attraction constants $(\Delta a)$ for the tautomers. They then used these values of $\Delta a$ and the van der Waals theory of fluids to predict $\Delta \bar{v}_{r x n}, K_{c}$, and the enthalpy change on reaction at temperatures and pressures other than those explored experimentally.

Finally, Peck et al. used the measured values of $\Delta \bar{v}_{r x n}$ to explore the amount of clustering of the solvent about the solutes. The average number, $n_{i}^{e}$, of solvent molecules clustered about a solute molecule $(i)$ in excess of the bulk value was determined from

$$
n_{i}^{e}=\rho\left(k_{B} T \kappa_{T}-\bar{v}_{i}^{\infty}\right)
$$

At a reduced temperature of 1.003 , the maximum differences in average solvent cluster sizes between the tautomers in difluoroethane and in propane were 94 and 76 molecules, respectively.

Gupta et al. (1993) have reported on chemical equilibrium related to hydrogen bonding in SCFs. They studied hydrogen bonding between methanol and triethylamine in $\mathrm{SC} \mathrm{SF}_{6}$ and found a pronounced solvent effect. The equilibrium constant for methanol-triethylamine association increased as the pressure decreased toward the critical point, and the effect of pressure was most pronounced near the critical point. The natural logarithm $K_{c}$ also varied with density, although the plots exhibited much less curvature than the pressure plots. The In $K_{c}$ vs. density plots were nearly linear, but the values of $K_{c}$ at $50^{\circ} \mathrm{C}$ and at densities below $8 \mathrm{~mol} / \mathrm{L}$ were higher than expected from the trends at higher densities. The authors attributed this added increase in hydrogen bonding to solute-solute clustering. The authors also modified the hydrogen-bonding lattice-fluid equation of state so that it included the solvent effect and gave a better description of the experimental observations.

O'Shea et al. (1991a) probed hydrogen bonding and polar interactions in $\mathrm{SC}$ ethane, fluoroform, and $\mathrm{CO}_{2}$ by measuring the azo-hydrazone tautomeric equilibrium of 4-(phenylazo)1-naphthol in these solvents. The less polar azo tautomer was dominant in ethane, nearly equal amounts of the two tautomers were in equilibrium in $\mathrm{CO}_{2}$, and the more polar hydrazone tautomer was dominant in fluoroform. These shifts in the equilibrium composition favoring the more polar tautomer were attributed to the large quadrupole moment of $\mathrm{CO}_{2}$ and the large dipole moment and hydrogen bond donor ability of fluoroform. The influence of pressure on hydrogen bonding and on specific polar interactions was different, suggesting that pressure can be used to control processes involving polar molecules in some SCFs.

Tautomeric equilibria have also been studied by Yagi et al. (1993) and Akao and Yoshimura (1991), who reported a keto-enol tautomerization of 2,4-pentanedione in $\mathrm{SC} \mathrm{CO}_{2}$ and $\mathrm{SC} \mathrm{CHF}_{3}$. Akao and Yoshimura (1991) sought, unsuc- cessfully, to detect a "critical anomaly" in the chemical equilibrium that had been reported to exist by Krichevskii et al. (1969). The data of Akao and Yoshimura (1991) showed that the equilibrium constant was a well-behaved function of density, even near the critical density. There was no evidence of any anomalies. We noted earlier that alternate explanations 'have emerged for Krichevskii's results and that those results cannot be used as evidence of critical anomalies. Yagi et al. (1993) observed that in nonpolar liquid $n$-hexane, 2,4-pentanedione exists in the less polar enol form, while slightly polar $\mathrm{CO}_{2}$ appears to favor the keto form, possibly because of its quadrupole moment, since it has no dipole moment. Moreover, a well-behaved shift in the tautomeric equilibrium from enol to keto was observed with an increase in the density of $\mathrm{SC} \mathrm{CO}_{2}$. This behavior was consistent with the results of Akao and Yoshimura (1991). Moreover, the dependence of $\log K_{c}$ (given as the absorption ratio of the two tautomers) on density was nearly linear. This density dependence is consistent with the experiments and analysis of Peck et al. (1989).

While the studies just discussed focused on using chemical equilibrium to investigate molecular interactions in SCFs, there has also been some work on chemical equilibrium centered on the practical application of SCF media for chemical reactions. Fluid phase equilibrium is also an important part of this work because the critical point of a mixture can vary widely with composition. Consequently, assignment of operating conditions on the basis of chemical equilibrium considerations alone could result in operation below the critical locus (subcritical conditions). See McHugh and Krukonis (1994), Subramaniam and McHugh (1986), and Brennecke and Eckert (1989a) for discussions of various types of phase behavior displayed in SC mixtures.

The components in and composition of an SCF reaction mixture dictate the region of the pressure-temperature space in which a single phase exists. At the same time, pressure and temperature also influence the reaction equilibrium. Thus, both chemical and phase equilibrium depend on the operating conditions, and a knowledge of how they vary with temperature and pressure is helpful for the proper interpretation of experimental kinetics data and the rational design of processes for conducting reactions at supercritical conditions. With this motivation, Saim and Subramaniam (1988) studied the effects of pressure, temperature, and initial mixture composition on equilibrium in an SCF medium by simultaneously solving reaction equilibrium and phase equilibrium equations. Reaction equilibrium was calculated by minimizing the Gibbs free energy. The Peng-Robinson equation of state was used for phase equilibrium calculations. The authors compared their equilibrium calculations with experimental data (though not necessarily at equilibrium) in the literature for the isomerization of $n$-hexane, the isomerization of 1 -hexene, and the oxidation of iso-butane in $\mathrm{SC} \mathrm{CO}_{2}$. The equilibrium conversions and yields calculated for the three reactions were always higher than the experimental values, and the authors concluded that this comparison showed their analysis could be consistent with the published data. In a subsequent study, Saim et al. (1989) presented experimental results for the equilibrium conversion of 1-hexene isomerization over $\mathrm{Pt} / \gamma$ $\mathrm{Al}_{2} \mathrm{O}_{3}$ in $\mathrm{SC} \mathrm{CO}_{2}$. At $265^{\circ} \mathrm{C}$ and subcritical pressures the combined conversion to 2-hexenes and to 3-hexenes was 69 and $21 \%$, respectively, whereas the calculated equilibrium 
values were 72.4 and $24.3 \%$. We discuss this work further in the subsection Heterogeneous Catalysis and Heterogeneous Reactions.

\section{Applications}

The research on the fundamentals of reactions in SCFs, which we reviewed in the previous section has been motivated, in part, by the existence of practical applications of SCFs as media for chemical reactions. These applications are reviewed in this section. Herein, we discuss Diels-Alder reactions, organometallic reactions, heterogeneously catalyzed reactions, fuels processing, oxidation chemistry, electrochemistry, biomass utilization, polymerization, and materials synthesis.

\section{Diels-Alder reactions}

Diels-Alder reactions are cycloaddition reactions of a conjugated diene with an alkene or alkyne. They have been used extensively to synthesize organic compounds such as insecticides, fragrances, plasticizers, and dyes (Paulaitis and Alexander, 1987). The 1 and 4 positions of the diene are the sites of the reaction. These reactions occur by a concerted bimolecular mechanism that is independent of the fluid phase in which the reaction occurs. Because these reactions have been studied extensively in the liquid phase, they are useful probes for studying the effects of SCF solvents on reaction kinetics.

The cycloaddition of isoprene to maleic anhydride in $\mathrm{SC}$ $\mathrm{CO}_{2}$ has been investigated by Paulaitis and Alexander (1987), Kim and Johnston (1987a), and Ikushima et al. (1991). Paulaitis and Alexander (1987) desired to learn how pressure could be used to control reaction rates in SCF reaction mixtures. They reported that the second-order rate constant increased with pressure. The effect of pressure was most pronounced near the critical point, however, where the activation volume assumed large negative values. Indeed, the rate

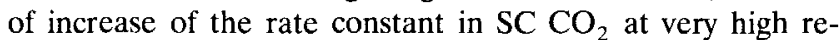
duced pressures was similar to that which they observed in three liquid solvents. Some of these results appeared in Figure 3. These results agreed with predictions Kim and Johnston (1987a) made about the effect of pressure on the rate constant. They used solvatochromic shift data for phenol blue and kinetics data in other solvents to develop a kinetics correlation after Eq. 21. They then used the correlation to predict the value of the rate constant in $\mathrm{SC} \mathrm{CO}_{2}$. They also used Eq. 22 to calculate the activation volume for this reaction. At $35^{\circ} \mathrm{C}$, the activation volume was $-4,000 \mathrm{~cm}^{3} / \mathrm{mol}$ at 75 bar, where $\mathrm{CO}_{2}$ was highly compressible, but at 300 bar the activation volume was only $-55 \mathrm{~cm}^{3} / \mathrm{mol}$. As a comparison, they noted that the activation volume for this reaction in liquid ethyl acetate at $35^{\circ} \mathrm{C}$ is $-37.4 \mathrm{~cm}^{3} / \mathrm{mol}$. The large negative activation volumes near the critical pressure were attributed to the partial molar volumes assuming large, negative values near the critical point. Paulaitis and Alexander (1987) also gave phase diagrams for the $\mathrm{CO}_{2}$-maleic anhydride and $\mathrm{CO}_{2}$-adduct systems. They developed a general analysis of reaction kinetics at near-critical conditions by combining geometric arguments about thermodynamics near critical points with transition-state theory. Ikushima et al. (1991) used high pressure FTIR spectroscopy for an in situ study of the aluminum chloride-catalyzed Diels-Alder reaction of isoprene and maleic anhydride. The results led the investigators to suggest that the reaction proceeded via a two-step mechanism under these conditions.

Kim and Johnston (1988) used the Diels-Alder cycloaddition of methyl acrylate to cyclopentadiene in $\mathrm{SC} \mathrm{CO}_{2}$ to demonstrate experimentally that the selectivity of a parallel reaction network can be controlled by adjusting the pressure. They reported the effect of pressure on the selectivity to the endo- and exo-adducts for reactions at 35 and $45^{\circ} \mathrm{C}$ in a pressure range of 80 to 300 bar. The endo- to exo-selectivity increased with pressure, but this increase was very small. The difference between the highest and lowest selectivities reported at a given temperature was about $2.5 \%$, and the authors estimated the uncertainty in a measured selectivity to be $1.0 \%$. The authors explained the slight increase in selectivity with pressure by noting that the endo-transition state has a larger dipole moment than does the exo-transition state. Consequently, the endo-transition state would interact more strongly with the solvent and be favored as the solvent density or polarity increased. The authors expressed this pressure effect on the selectivity in terms of the difference in the partial molar volumes of the endo- and exo-transition states. The authors also correlated the endo- to exo-selectivity with the transition energy, $E_{T}$, for phenol blue, and then used this correlation to predict the selectivity in the near-critical region.

Ikushima et al. $(1990,1992)$ reported kinetics data for the Diels-Alder addition of methyl acrylate to isoprene in $\mathrm{SC} \mathrm{CO}_{2}$ at $323 \mathrm{~K}$ and pressures between 4.9 and $20.6 \mathrm{MPa}$. The rate constant increased with pressure, and the selectivity to the two different adducts (methyl 4-methyl-3-cyclohexene-1carboxylate and methyl 3-methyl-3-cyclohexene-1-carboxylate) was also pressure dependent. The production of the second adduct was greatest near the critical pressure of $\mathrm{CO}_{2}$, and it decreased as the pressure increased further. This observation was attributed to the local solvent density around a solute exceeding the bulk density (molecular charisma). This clustering of solvent molecules around the transition complex in the critical region was thought to produce steric constraints that favored the production of one of the isomers. They found that the rate constant at $323 \mathrm{~K}$ correlated with the transition energy, $E_{T}(30)$, of pyridinium $N$-phenoxide betaine dye in accord with Eq. 21 as shown in Figure 14. The authors calculated the activation volume at $323 \mathrm{~K}$ (from Eq. 22) to be about $-700 \mathrm{~cm}^{3} / \mathrm{mol}$ near the critical pressure. The absolute value of the activation volume was about an order of magnitude smaller at pressures further from the critical pressure. Ikushima et al. (1992) used a method developed by Debenedetti (1987) to determine that about ten solvent molecules clustered around the transition state near the critical point. The cluster size was less than two for pressures further from $P_{c}$. Figure 15 summarizes these results. This work was consistent with earlier reports demonstrating the potential use of pressure as a lever for manipulating reaction kinetics and selectivity in fluids near critical points. These pressure effects are most pronounced near the critical region.

Issacs and Keating (1992) briefly reported kinetic measurements for the Diels-Alder reaction between $p$-benzoquinone and cyclopentadiene in $\mathrm{CO}_{2}$ for temperatures between 25 and $40^{\circ} \mathrm{C}$ and pressures between 60 and 240 bar. The rate constant increased with pressure, and a plot of the rate as a 


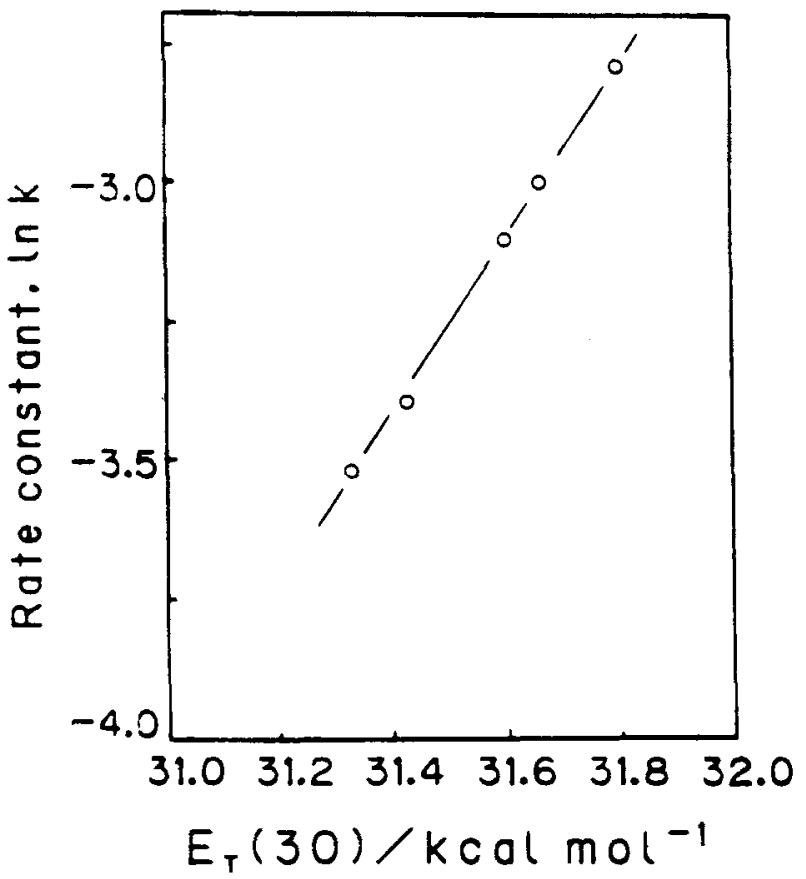

Figure 14. Rate constant for cycloaddition of isoprene and methyl acrylate in $\mathrm{SC} \mathrm{CO}_{2}$ at $323 \mathrm{~K}$ correlated with $E_{T}(30)$ (Ikushima et al., 1992).

Courtesy of American Chemical Society.

function of temperature at constant density showed no discontinuity at the critical temperature. That is, there were no "anomalies" near the critical point.

\section{Organometallic reactions}

Organometallic compounds are important in many catalytic reactions, and volatile organometallics can be used in

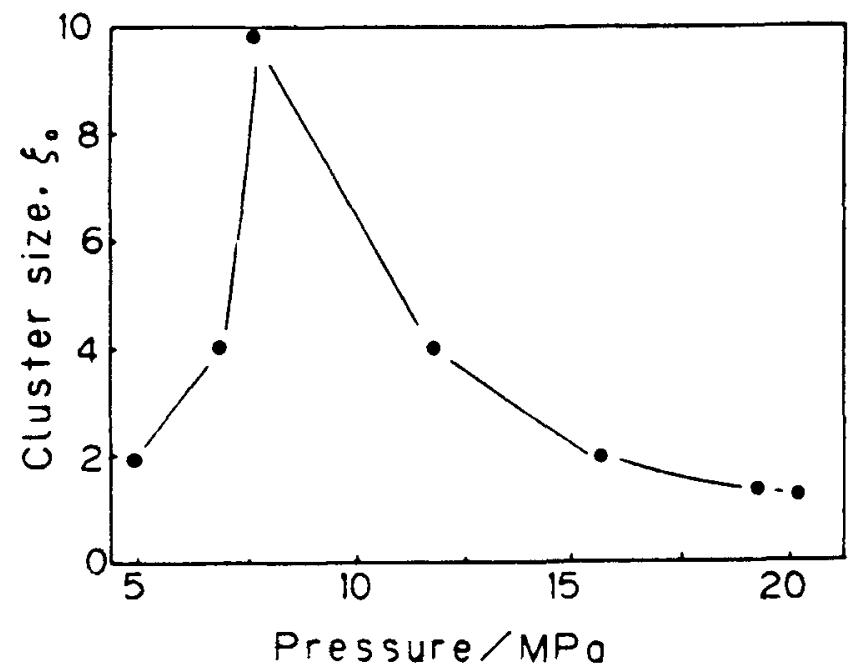

Figure 15. Cluster size for cycloaddition of isoprene and methyl acrylate in $\mathrm{SC} \mathrm{CO}_{2}$ at $323 \mathrm{~K}$ (Ikushima et al., 1992).

Courtesy of American Chemical Society. chemical vapor deposition processes to grow thin films for microelectronics applications. The use of SCFs (primarily $\mathrm{CO}_{2}$ and $\mathrm{Xe}$ ) as media for reactions of organometallic species has been explored as a means to synthesize new compounds, to improve syntheses of existing compounds, to gain a better understanding of fundamental organometallic chemistry, and to improve commercial processes for homogeneously catalyzed reactions. Among the useful properties of $\mathrm{SC} \mathrm{CO}_{2}$ and $\mathrm{Xe}$ are their chemical inertness and their complete miscibility with permanent gases such as $\mathrm{N}_{2}$ and $\mathrm{H}_{2}$, which are often used in organometallic reactions. The miscibility of permanent gases is worthy of emphasis because the low solubility of these gases in liquid solvents limits the generation of potentially useful organometallic compounds and affects the rate of some homogeneously catalyzed reactions. In addition, since $\mathrm{Xe}$ is completely transparent to UV, visible, and mid-IR radiation, it is especially attractive for use in in situ spectroscopic studies of organometallic chemistry. Liquid Xe has been used in organometallic syntheses, but the maximum usable temperature is low (about $-40^{\circ} \mathrm{C}$ ), as is the solubility of some carbonyl-containing organometallic precursors.

Poliakoff's group at the University of Nottingham has explored several organometallic systems in SCFs (Poliakoff et al., 1988, 1991; Howdle et al., 1992). In one project, they started with metal carbonyls and photochemically replaced the $\mathrm{CO}$ with $\mathrm{N}_{2}$ or $\mathrm{H}_{2}$ (Howdle and Polaikoff, 1989; Howdle et al., 1989, 1990). Performing these syntheses in SC Xe allowed high $\mathrm{N}_{2}$ and $\mathrm{H}_{2}$ concentrations in the reacting mixture, which facilitated the production and stabilization of previously unknown dinitrogen and dihydrogen complexes. The progress of the reactions was, at times, followed using time-resolved infrared spectroscopy. For example, Howdle and Poliakoff (1989) studied the UV photolysis of $\eta^{5}$ $\mathrm{C}_{5} \mathrm{H}_{5} \mathrm{Mn}(\mathrm{CO})_{3}$ and $\eta^{5}-\mathrm{C}_{5} \mathrm{H}_{5} \mathrm{Re}(\mathrm{CO})_{3}$ in SC Xe pressurized with $\mathrm{H}_{2}$. The two organometallics reacted differently. The reactant containing $\mathrm{Mn}$ formed a nonclassical dihydrogen complex, $\eta^{5}-\mathrm{C}_{5} \mathrm{H}_{5} \mathrm{Mn}(\mathrm{CO})_{2}\left(\mathrm{H}_{2}\right)$, whereas the $\mathrm{Re}$ compound formed a dihydride, $\eta^{5}-\mathrm{C}_{5} \mathrm{H}_{5} \mathrm{Re}(\mathrm{CO})_{2} \mathrm{H}_{2}$. Moreover, the $\eta^{5}$ $\mathrm{C}_{5} \mathrm{H}_{5} \mathrm{Mn}(\mathrm{CO})_{2}\left(\mathrm{H}_{2}\right)$ is stabilized by the high concentration of $\mathrm{H}_{2}$ in the supercritical fluid. Replacing the $\mathrm{H}_{2}$ with $\mathrm{N}_{2}$ results in rapid exchange of the $\mathrm{H}_{2}$ in the dihydrogen complex with $\mathrm{N}_{2}$. Howdle et al. (1989) synthesized $\eta^{5}$ $\mathrm{C}_{5} \mathrm{H}_{5} \mathrm{Re}(\mathrm{CO})_{3-x}\left(\mathrm{~N}_{2}\right)_{x}$, where $x=1,2$, or 3 , from the UV photolysis of $\eta^{5}-\mathrm{C}_{5} \mathrm{H}_{5} \mathrm{Re}(\mathrm{CO})_{3}$ in SC Xe doped with $\mathrm{N}_{2}$. The dinitrogen compound $(x=2)$ was stable at room temperature. In a subsequent report, Howdle et al. (1990) extended their previous work by synthesizing $\left(C_{n} R_{n}\right) \mathrm{M}(\mathrm{CO})_{2}\left(\mathrm{~N}_{2}\right)$ and $\left(C_{n} R_{n}\right) M(\mathrm{CO})_{2}\left(\mathrm{H}_{2}\right)$ complexes $[M=\mathrm{Fe}, \mathrm{Mn}, \mathrm{Re}, \mathrm{Cr} ; R=\mathrm{H}$ for $n=4 ; R=\mathrm{H}$ or Me for $n=5$ or 6] from UV photolysis of a series of $\left(C_{n} R_{n}\right) M(\mathrm{CO})_{3}$ compounds in SC Xe doped with $\mathrm{H}_{2}$ or $\mathrm{N}_{2}$.

In another project, Poliakoff's group has explored the photochemical activation of $\mathrm{C}-\mathrm{H}$ bonds by organometallic complexes in SCFs (Jobling et al., 1990a, b). SC $\mathrm{CO}_{2}$ was an attractive medium for C-H bond activation because it offered the required chemical inertness and miscibility with low molecular weight alkanes. Jobling et al. (1990a) showed that UV photolysis of $\eta^{5}-\mathrm{C}_{5} \mathrm{Me}_{5} \mathrm{Ir}(\mathrm{CO})_{2}$ in $\mathrm{SC} \mathrm{CO}_{2}$ containing $\mathrm{RH}$, where $R=\mathrm{H}$ or $\mathrm{C}_{2} \mathrm{H}_{5}$, formed $\eta^{5}-\mathrm{C}_{5} \mathrm{Me}_{5} \operatorname{Ir}(\mathrm{CO})(\mathrm{H}) R$. They also showed (Jobling et al., 1990b) that C-H bonds in polyethylene could be activated by using $\mathrm{SC} \mathrm{CO}_{2}$ to im- 
pregnate the polymer with $\mathrm{C}_{5} \mathrm{Me}_{5} \mathrm{Ir}(\mathrm{CO})_{2}$, conducting the UV photolysis, and then extracting the unreacted $\mathrm{C}_{5} \mathrm{Me}_{5} \operatorname{Ir}(\mathrm{CO})_{2}$.

Rathke et al. $(1991,1992)$ examined the kinetics and thermodynamics associated with olefin hydroformylation reactions catalyzed by cobalt carbonyl compounds in $\mathrm{SC} \mathrm{CO}_{2}$. These hydroformylation reactions are accomplished industrially as homogeneously catalyzed processes in liquid solvents. The process involves the reaction of gases $\left(\mathrm{CO}\right.$ and $\left.\mathrm{H}_{2}\right)$ with soluble substrates. Rathke et al. speculated that using SCF media for this reaction could overcome difficulties associated with gas-liquid mixing, diffusion across a gas-liquid interface, and downstream separation and recovery of the catalyst in conventional liquid media. They noted that an SCF medium could provide a single, homogeneous phase for the reaction, and that the reactive gas concentrations could be much higher in $\mathrm{SC} \mathrm{CO}_{2}$ than in a liquid solvent. They also forecast that catalyst and product separations, normally accomplished by distiliation, could be done more efficiently by altering the pressure of the SCF. Rathke et al. used in-situ high-pressure ${ }^{1} \mathrm{H},{ }^{13} \mathrm{C}$, and ${ }^{59} \mathrm{Co}$ nuclear magnetic resonance (NMR) measurements to follow the progress of the reaction. They pointed out that SCFs are particularly advantageous for high-pressure NMR experiments because their provision of a single fluid phase obviates the need for stirring and their low viscosities (compared to a liquid) lead to narrow linewidths for quadrupolar nuclei such as ${ }^{59} \mathrm{Co}$.

Rathke et al. reported the rate constants and equilibrium constant for the hydrogenation of dicobalt octacarbonyl, $\mathrm{Co}_{2}(\mathrm{CO})_{8}+\mathrm{H}_{2}=2 \mathrm{HCO}(\mathrm{CO})_{4}$, in $\mathrm{SC} \mathrm{CO}$ at temperatures between 60 and $180^{\circ} \mathrm{C}$. This reaction is one of the steps in the hydroformylation of olefins. A van't Hoff plot of the equilibrium data gave the reaction enthalpy and entropy as $4.7 \pm$ $0.2 \mathrm{kcal} / \mathrm{mol}$ and $4.4 \pm 0.5 \mathrm{kcal} / \mathrm{mol} \cdot \mathrm{K}$, respectively. All of the kinetics and thermodynamics data were in good agreement with published values for this reaction in nonpolar hydrocarbon solvents such as $n$-heptane and methylcyclohexane. They also reported the "steady-state" concentrations of the cobalt carbonyl compounds in the reaction above and the rate of aldehyde formation during the hydroformylation of an olefin in $\mathrm{SC} \mathrm{CO}_{2}$. These data also compared favorably with literature values for hydroformylation in nonpolar liquid media. The authors concluded that cobalt-catalyzed hydroformylation worked well in $\mathrm{SC} \mathrm{CO}_{2}$ and that the reation kinetics and thermodynamics behaved normally.

In another report of homogeneous catalysis in $\mathrm{SC} \mathrm{CO}_{2}$ Jessop et al. (1994) showed that formic acid could be produced from $\mathrm{H}_{2}$ and $\mathrm{CO}_{2}$ using a catalytic ruthenium phosphine complex. In this case, $\mathrm{CO}_{2}$ serves as both a reactant and a solvent. Jessop et al. found that the reaction rate was 18 times faster in $\mathrm{SC} \mathrm{CO}_{2}$ than in liquid tetrahydrofuran at otherwise identical conditions. The rate increase was attributed to the same features of SCF reaction media identified by Rathke et al. (1991, 1992) and discussed earlier.

\section{Heterogeneous catalysis and heterogeneous reactions}

In this section we review published accounts of heterogeneous catalytic reactions at supercritical conditions. We discuss studies on isomerization, Fischer-Tropsch synthesis, enzyme-catalyzed reactions, and then other catalytic reactions.
We defer a discussion of heterogeneously catalyzed oxidation reactions until the section titled Oxidation Reactions, which deals more generally with oxidation reactions.

Isomerization reactions and catalyst deactivation. In their review of reactions in SCFs, Subramaniam and McHugh (1986) highlighted the work of Tiltscher et al. (1981, 1984), which demonstrated the potential utility of SCFs as media for heterogeneous catalytic reactions by comparing results obtained for identical reactions in the gas, liquid, and SCF phases. The work of Tiltscher's group (see Tiltscher and Hoffman, 1987) with 1-hexene isomerization on a low-activity, macroporous $\alpha-\mathrm{Al}_{2} \mathrm{O}_{3}$ showed that the initial ratio of cis/trans-2-hexene formed could not be influenced by temperature in the gas phase. There was a modest effect of both temperature and pressure on this ratio for reactions in the liquid phase, but there was a more pronounced effect in the SCF phase. Tiltscher et al. (1984) also showed that catalyst deactivation occurred during the gas-phase reaction because hexene oligomers with low volatilities deposited on the catalytically active surface. Performing the reaction at the same temperature, but above the critical pressure prevented the deposition of these oligomers and the ensuing catalyst deactivation. Thus, one advantage of conducting heterogeneous catalytic reactions at $\mathrm{SC}$ conditions is the possibility of doing in-situ extraction of coke precursors.

Subramaniam and coworkers (Saim et al, 1989; Saim and Subramaniam, 1988, 1990, 1991; Baptist-Nguyen and Subramaniam, 1992; Subramaniam and McCoy, 1994; McCoy and Subramaniam, 1994; Ginosar and Subramaniam, 1994) greatly extended this work on 1-hexene isomerization by investigating the reaction over a high-activity, microporous, industrial $\mathrm{Pt} / \gamma-\mathrm{Al}_{2} \mathrm{O}_{3}$ catalyst and using $\mathrm{CO}_{2}$ as a diluent in the reactor. They analyzed the phase and reaction equilibria to establish the thermodynamic constraints (equilibrium conversions, region for single-phase operation) in this system (Saim and Subramaniam, 1988), and they reported new experimental data from both batch and continuous reactors. The conversions achieved in the batch experiments confirmed the authors' equilibrium analysis. The continuous runs were used to assess catalyst deactivation in hexene- $\mathrm{CO}_{2}$ mixtures. At a subcritical pressure, the activity of the catalyst decreased (presumably due to coking), whereas at a nearly identical temperature but supercritical pressure no loss of catalyst activity was observed. Saim et al. (1989) attributed this catalyst activity maintenance to the solvent power of the dense SCF, which presumably prevented the deposition of higher molecular weight oligomers within the catalyst pores. These oligomers were thought to be coke precursors, and their extraction by the SCF was supported by the brownish color of the reactor effluent at supercritical conditions as opposed to the clear effluent obtained at subcritical conditions. These results highlight a possible advantage of SCF technology over conventional processes in some heterogeneous catalytic applications.

Saim and Subramaniam (1990, 1991) observed that the end-of-run isomerization rates decreased with isothermal increases in pressure in the subcritical region but increased with pressure in the supercritical region. In sharp contrast to the activity maintenance observed by Tiltscher and coworkers in a macroporous catalyst, the microporous $\mathrm{Pt} / \gamma-\mathrm{Al}_{2} \mathrm{O}_{3}$ catalyst used by Saim and Subramaniam deactivated even at SC con- 
ditions. A significant portion of the catalyst activity was lost due to the buildup of unextractable coke in the catalyst pores during the subcritical phase of reactor fill-up. In related work that was consistent with that of Subramaniam and coworkers, Manos and Hofmann (1991) concluded that complete in-situ reactivation of a microporous zeolite catalyst by SCFs is impossible. This conclusion was based on coke desorption rates and the solubilities of model coke compounds in SCFs. The catalyst deactivation rate can be reduced at SC conditions, however, because freshly formed coke precursors can be dissolved by the SCF reaction medium.

Employing a single-pore model to describe the physicochemical processes underlying coke formation and in-situ coke extraction, Baptist-Nguyen and Subramaniam (1992) showed that for an increase in pressure along a near-critical isotherm, there exists an optimum pressure (i.e., reaction mixture density) at which catalyst activity is maximized. At lower than optimum densities the catalyst undergoes deactivation due to a lack of coke extraction, while at higher than optimum densities the catalyst activity decreases due to pore-diffusion limitations in the liquidlike reaction mixtures (see Figure 16). Ginosar and Subramaniam (1994) confirmed these qualitative predictions by investigating 1-hexene isomerization at reaction mixture densities ranging from gaslike to liquidlike values. At a space velocity of $135 \mathrm{~g}$ hexene/g cat $/ \mathrm{h}, 8$-h isomerization rates in near-critical $\left(\rho_{r}=0.85\right) \mathrm{mix}-$ tures were nearly twice as high and deactivation rates were threefold lower than the corresponding rates in subcritical $\left(\rho_{r}=0.42\right)$ mixtures (Figure 17a). As shown in Figure 17b, near-critical reaction mixtures were better able to maintain the accessible surface area and pore volume since coke lay-

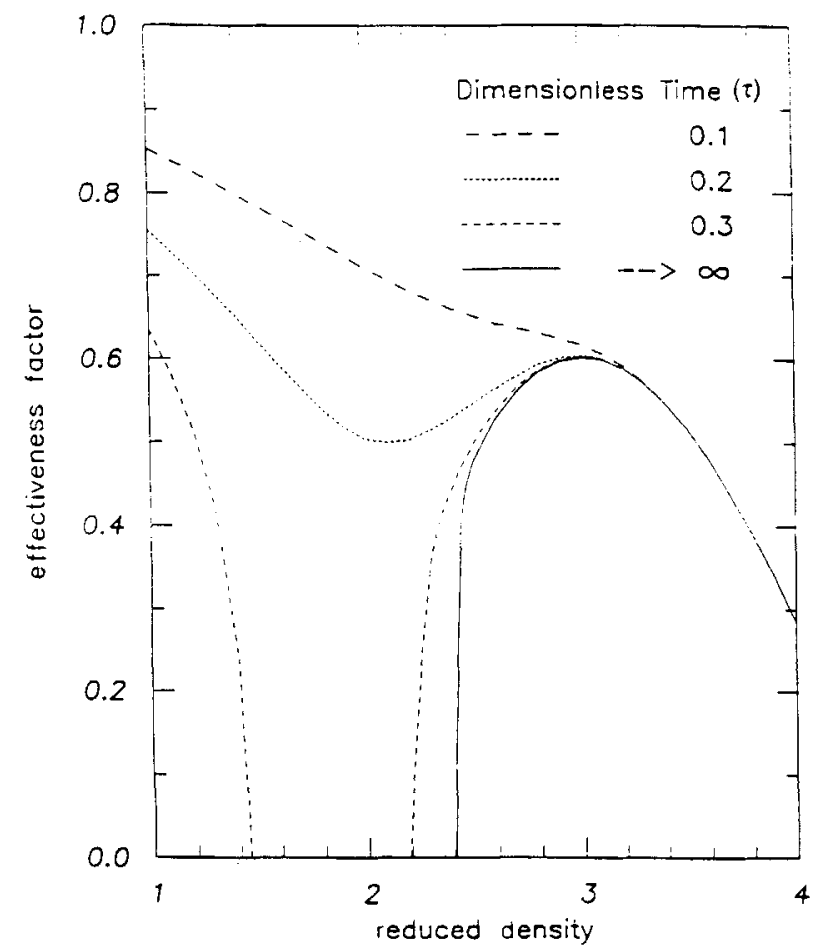

Figure 16. Effectiveness factor map for 1-hexene isomerization at SC conditions (Baptist-Nguyen and Subramaniam, 1992).

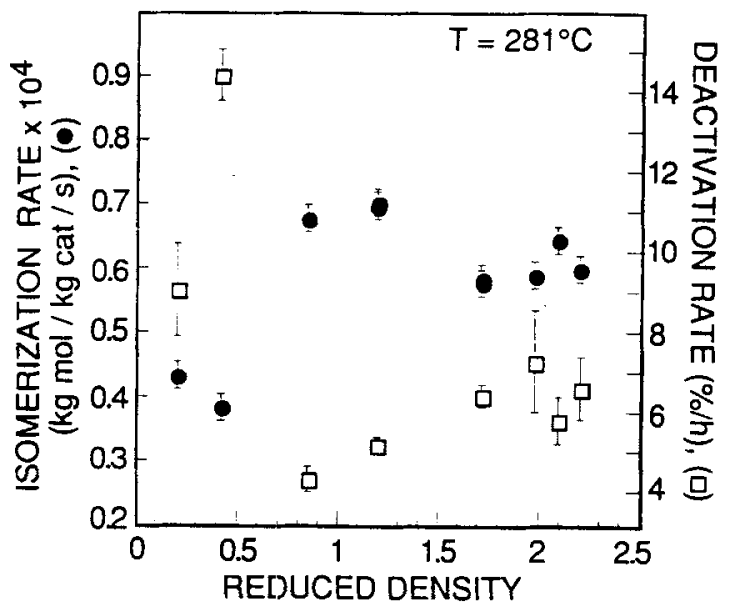

(a)

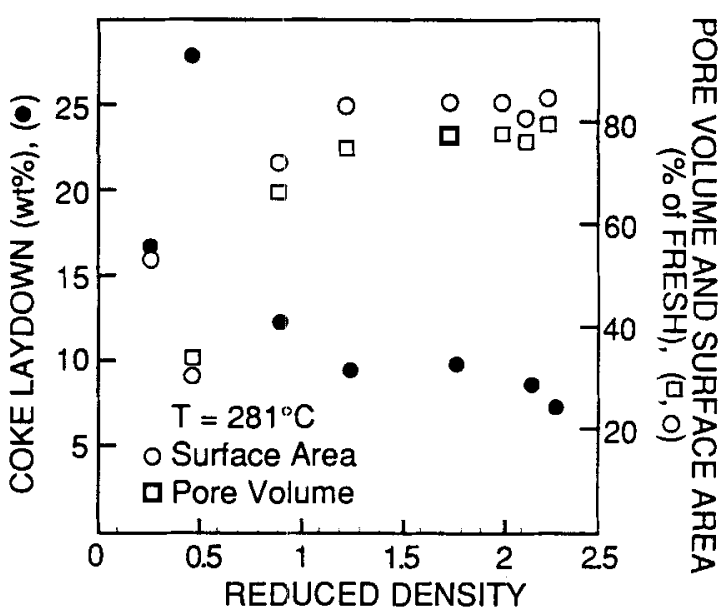

(b)

Figure 17. End-of-run results from 1-hexene isomerization at different sub- and supercritical densities (Ginosar and Subramaniam, 1994).

(a) Isomerization rates and deactivation rates. (b) Coke laydown, pore volume, and surface area.

down was about one-half the amount observed at subcritical conditions.

Saim and Subramaniam (1991) and Ginosar and Subramaniam (1994) also found that the in situ extraction of the coke compounds by near-critical and supercritical reaction mixtures prevents pore plugging that otherwise occurs at subcritical conditions. Although the coke laydown decreased at supercritical conditions, the isomerization rates were lower and deactivation rates were higher due to pore diffusion limitations in the liquidlike reaction mixtures. The authors therefore concluded that near-critical reaction mixtures provide an optimum combination of solvent and transport properties that is better than either subcritical (gaslike) or dense supercritical (liquidlike) mixtures for maximizing the isomerization rates and for minimizing catalyst deactivation rates. These findings point out that catalytic reactions require liquidlike reaction media for coke extraction and/or heat removal, yet gaslike diffusivities for enhanced reaction rates can benefit from the use of near-critical reaction media. 
More recently, Subramaniam and McCoy (1994) presented a kinetic scheme that describes the formation of coke compounds from olefinic oligomers and their reversible adsorption/desorption at the catalyst surface. Using this kinetic scheme, McCoy and Subramaniam (1994) report a model based on continuous mixture kinetics to interpret the molecular weight distributions for the olefinic oligomers and aromatic coke compounds that are observed experimentally. The model is consistent with observations of decreased catalyst deactivation rates when coke desorption rates approach coke production rates, and it also predicts catalyst activity maintenance at supercritical conditions.

Amelse and Kutz (1991) described a patented catalytic process to isomerize a mixture of xylenes to a product stream enriched in $p$-xylene at conditions beyond the critical point of the mixture. Operating at supercritical conditions provided enhanced catalyst activity compared to the conventional liquid-phase catalytic process that operated at lower temperatures. Moreover, operating at SC conditions reduced catalyst deactivation compared to the conventional gas-phase process. Reducing catalyst deactivation allowed elimination of the $\mathrm{H}_{2}$ and related compression equipment that was required to maintain catalyst activity in the gas-phase process.

Fischer-Tropsch Synthesis. Fischer-Tropsch synthesis provides a means to produce higher hydrocarbons in the liquidfuel range from synthesis gas ( $\mathrm{CO}$ and $\mathrm{H}_{2}$ ). The catalysts used are typically based on iron or cobalt. The reaction has been studied since at least the $1920 \mathrm{~s}$, but it has been implemented on a commercial scale only in exceptional instances (World War II Germany, South Africa). Typically, economic factors favor more traditional sources of liquid fuels rather than Fischer-Tropsch synthesis.

The traditional Fischer-Tropsch synthesis route involves a gas-phase reaction. The reaction is exothermic, and heat removal issues present difficulties. Additionally, the higher hydrocarbons formed during the synthesis reaction may condense within the catalyst pores and lead to deactivation of the catalyst. These considerations have led to the exploration of the feasibility of a liquid-phase Fischer-Tropsch synthesis. Heat transfer improves in a liquid phase, and the higher hydrocarbons have higher solubilities in a liquid, but the lower diffusivity in the liquid-phase leads to transport limitations and slower overall reaction rates. These factors have motivated research into conducting Fischer-Tropsch synthesis in an SCF phase (Fan et al., 1991, 1992; Yokota et al., 1989, 1991; Yokota and Fujimoto, 1991). The advantages of an SCF in this application are its gaslike diffusivity and liquidlike solubility. Thus, it could combine the desirable features of the gas- and liquid-phase synthesis routes explored previously.

Fujimoto and coworkers used $n$-hexane as the reaction medium, and they compared Fischer-Tropsch synthesis in a gas-phase, in liquid $n$-hexane, and in SC $n$-hexane. They found that although the conversion of $\mathrm{CO}$ for the SCF phase synthesis fell between the conversions in the gas- and liquidphase reactions, the total amount of products from the SCFphase reaction was greater than that from the gas-phase reaction because of reduced coking and plugging of catalyst pores and reduced production of $\mathrm{CO}_{2}$ (Yokota et al., 1991). The phase in which the reaction was conducted influenced the product distribution. The SCF-phase reaction produced less methane, probably because of better distribution of heat in the reactor (Yokota et al., 1989). It also produced more long-chain olefins, probably because of the enhanced solubility of these higher hydrocarbons in the SCF. During gas-phase synthesis, these olefins might remain within the catalyst pores and they might be hydrogenated to paraffins (Fan et al., 1991). Yokota and Fujimoto (1991) also examined the effect of the catalyst pore size on the reaction rate and product distribution. For a given total pore volume, as the pore diameter decreases, the surface area increases, but the diffusivity within the pores decreases. These two competing effects lead to an optimum pore size for many catalytic reactions. The optimum pore diameter for $\mathrm{CO}$ conversion was about $10 \mathrm{~nm}$ for a ruthenium catalyst supported on $\gamma$-alumina. Yokota and Fujimoto (1991) also observed that although smaller catalyst pores led to higher rates, they produced a larger quantity of small olefins instead of the desired long-chain olefins.

Fischer-Tropsch synthesis in an SCF has advantages over the traditional gas-phase reaction and the experimental liquid-phase reaction. These advantages can be attributed to the SCF offering high diffusivities and improved heat transfer (relative to a liquid) and high solubilities (relative to a gas). The supercritical phase reaction also reduced production of undesired products.

Enzyme-Catalyzed Reactions. Enzymes can retain their activity and stability in nonaqueous environments. Consequently, they can be used to catalyze reactions in organic solvents and other nonconventional media. This discovery motivated a great deal of research, beginning in the early 1980s, aimed at better understanding the role of the solvent in enzyme catalysis and at developing new biocatalytic processes for the synthesis of chemicals. This attempt to identify solvent effects on enzyme catalyzed reactions and manipulate them to achieve a desired goal has been termed "solvent engineering" (Kamat et al., 1992, 1993). It is within this context that supercritical fluids have been examined as potential media for enzyme catalysis. Russell et al. (1994) recently provided a brief overview of work in this field. The attractive features of SCFs as media for biocatalysis include their diffusivities being higher (Russell and Beckman, 1991b) and viscosities being lower than those in liquid solvents, their pressure- and temperature-dependent solvent properties near the critical point, and their potential simplification of downstream processing (product separation and recovery).

The earliest reported demonstrations of enzyme activity and stability in SCFs were by Randolph et al. (1985), Hammond et al. (1985), and Nakamura et al. (1986). It is wellestablished that some water must be present in the system for enzymes to be catalytically active in an SCF. Therefore, the enzyme-catalyzed reaction may occur in the water film surrounding the enzyme instead of the continuous SCF phase (Krukonis and Hammond, 1988). $\mathrm{SC} \mathrm{CO}_{2}$, either pure or with a cosolvent, has been employed as the SCF solvent in nearly all investigations. The few exceptions are Hammond et al. (1985) using fluoroform, and work at the University of Pittsburgh (Kamat et al., 1992, 1993) that employed SC ethane, ethylene, $\mathrm{SF}_{6}$, and $\mathrm{CHF}_{3}$. Several different enzymes have been shown to maintain their activity after exposure to $\mathrm{SC} \mathrm{CO}_{2}$, pure and with added water and ethanol (Taniguchi et al., 1987). The use of SCFs implies elevated pressures, and high pressure can affect enzymes. The activity of some enzymes was reportedly adversely affected by depressurization (Kasche 
et al., 1988), but more recent work (Zagrobelny and Bright, 1992a) showed that denaturation occurred during compression, not during decompression when SCF solvents were employed.

Several different reactions have been examined to date. These include the oxidation of substituted phenols by polyphenol oxidase (Hammond et al,, 1985), the conversion of $p$-nitrophenylphosphate to $p$-nitrophenol by an alkaline phosphatase (Randolph et al., 1985), the oxidation of cholesterol by cholesterol oxidase (Randolph et al., 1988a,b,c), and the synthesis of aspartame precursors by thermolysin (Kamihira et al., 1987b). The classes of reactions studied most extensively in SCF solvents, however, have been esterification, interesterification, and transesterification reactions catalyzed by lipases. This extensive research into these systems has identified and elucidated the effects of many of the important variables, such as pressure (Miller et al., 1990, 1991; Erickson et al., 1990; Ikushima et al., 1993). Reaction rates in $\mathrm{SC} \mathrm{CO}_{2}$ and in organic solvents (typically hexane) have been compared (Martins et al., 1991; Bolz et al., 1991; Marty et al., 1990, 1992a, b; Kamat et al., 1992, 1993; Chi et al., 1988; Pasta et al., 1989). Kinetics parameters and reaction mechanisms have been reported (Miller et al., 1991; Bolz et al., 1991; Marty et al., 1992b; Dumont et al., 1992). The use of classical thermodynamics as a guide to understanding the effects of different solvents has been explored (Shen et al., 1992). The role of diffusion within the pores of immobilized enzyme particles has been found to be significant (Dumont et al., 1992; Bernard et al., 1992). Reported effectiveness factors ranged between 0.36 and 0.60 . The water content is an important variable, and it can influence the reaction rate (Miller et al., 1990, 1991; Chi et al., 1988; Marty et al., 1990; Yu et al., 1992; Dumont et al., 1991, 1992, 1993; Nakamura et al., 1990; Aaltonen and Rantakylä, 1991a). The addition of small amounts of water initially improved the rate because the enzyme requires a microaqueous environment to be active. Adding more than the amount of water required to form this microaqueous environment, however, leads to a decrease in the rate as shown in Figure 18. Presumably, too much water leads to the formation of an aqueous layer around the enzyme that hinders the transport of reactants and products from the SCF phase to the enzyme-containing phase. Marty et al. (1992a) advanced the idea that the water content that influenced the rate was the water content associated with the solid enzyme phase, and not simply the nominal water content of the SCF phase, which was the quantity typically reported. They measured adsorption isotherms for water and related the water content in the SCF phase to that in the enzyme-containing phase. To achieve the desired optimum amount of water in the enzyme microaqueous environment, one would need to add more water to SCF solvents with a high solubility for water than to SCFs that were more hydrophobic. Figure 19 shows that the optimum water content of the support is essentially the same for a reaction in $n$ hexane and in $\mathrm{SC} \mathrm{CO}_{2}$. Note that the water loading of the solvent required to achieve the optimal water content of the support would be very different in these two solvents.

One intriguing recent application of lipase-catalyzed reactions in SCF or liquid organic solvents is to control stereoselectivity during the synthesis of biologically active chiral compounds (Shen et al., 1992; Ikushima et al., 1993; Aaltonen

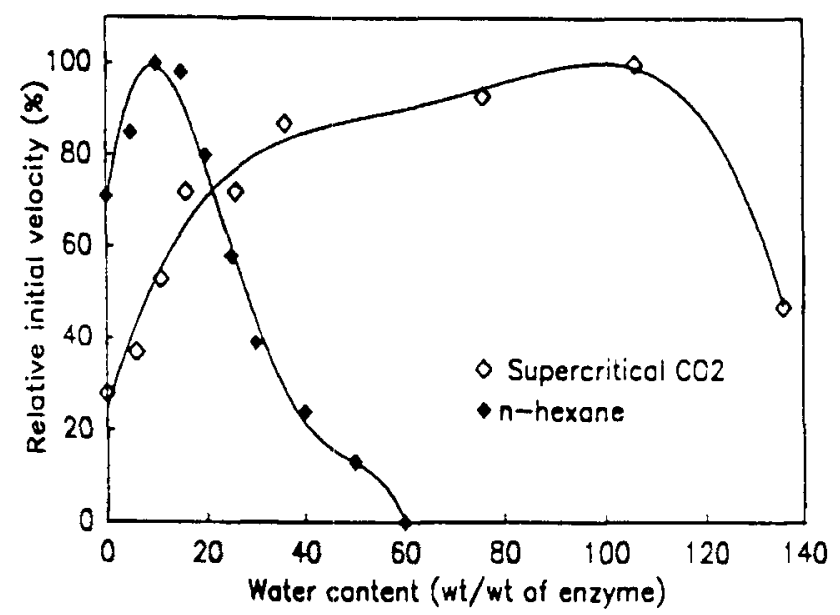

Figure 18. Effect of water content of fluid phase on enzyme-catalyzed reaction rate in $\mathrm{SC} \mathrm{CO}_{2}$ and $n$-hexane at $40^{\circ} \mathrm{C}$ (Marty et al., 1990).

Courtesy of Chapman \& Hall Publishers

and Rantakylä, 1991b; Martins et al., 1991). For example, ibuprofen, an anti-inflarnmatory drug, is used as a racemate. Only the $S(-)$ enantiomer is physiologically active, however. The $R(+)$ enantiomer is inactive. Aaltonen and Rantakylä (1991b) reported work on the resolution of racemic ibuprofen by enantioselective esterification reactions in $\mathrm{SC} \mathrm{CO}_{2}$ using lipases. The goal was to produce ibuprofen derivatives enantioselectively so they could then be separated by conventional means. These workers reported enantiomeric purities exceeding $90 \%$ for ester derivatives at an ibuprofen conversion of $25 \%$.

Biocatalysis in SCFs is a relatively new endeavor, but the knowledge base required for a fundamental understanding of the role and effects of the SCF is beginning to emerge. The pioneering work of Randolph et al. (1988a,b,c), for example, examined the effects of different cosolvents on the oxidation of cholesterol in $\mathrm{SC} \mathrm{CO}_{2}$. They found that branched butanols and ethanol enhanced the oxidation rate, whereas $n$-butanol, acetone, and methanol afforded much less dramatic enhancements or even diminished the reaction rate. They noted that these cosolvent effects could not be attributed to differences

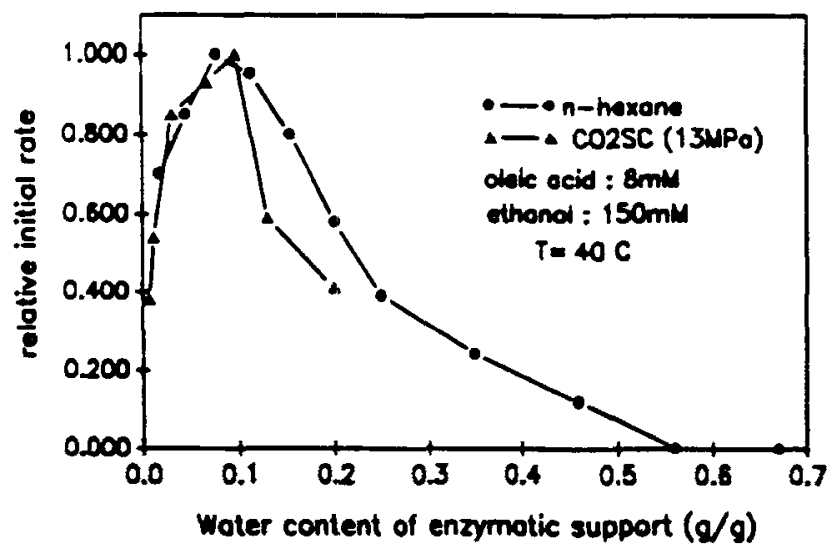

Figure 19. Effect of water content of enzymatic support on enzyme activity in $\mathrm{SC} \mathrm{CO}_{2}$ and $n$-hexane at $40^{\circ} \mathrm{C}$ (Marty et al., 1992).

Courtesy of John Wiley \& Sons, Inc. 


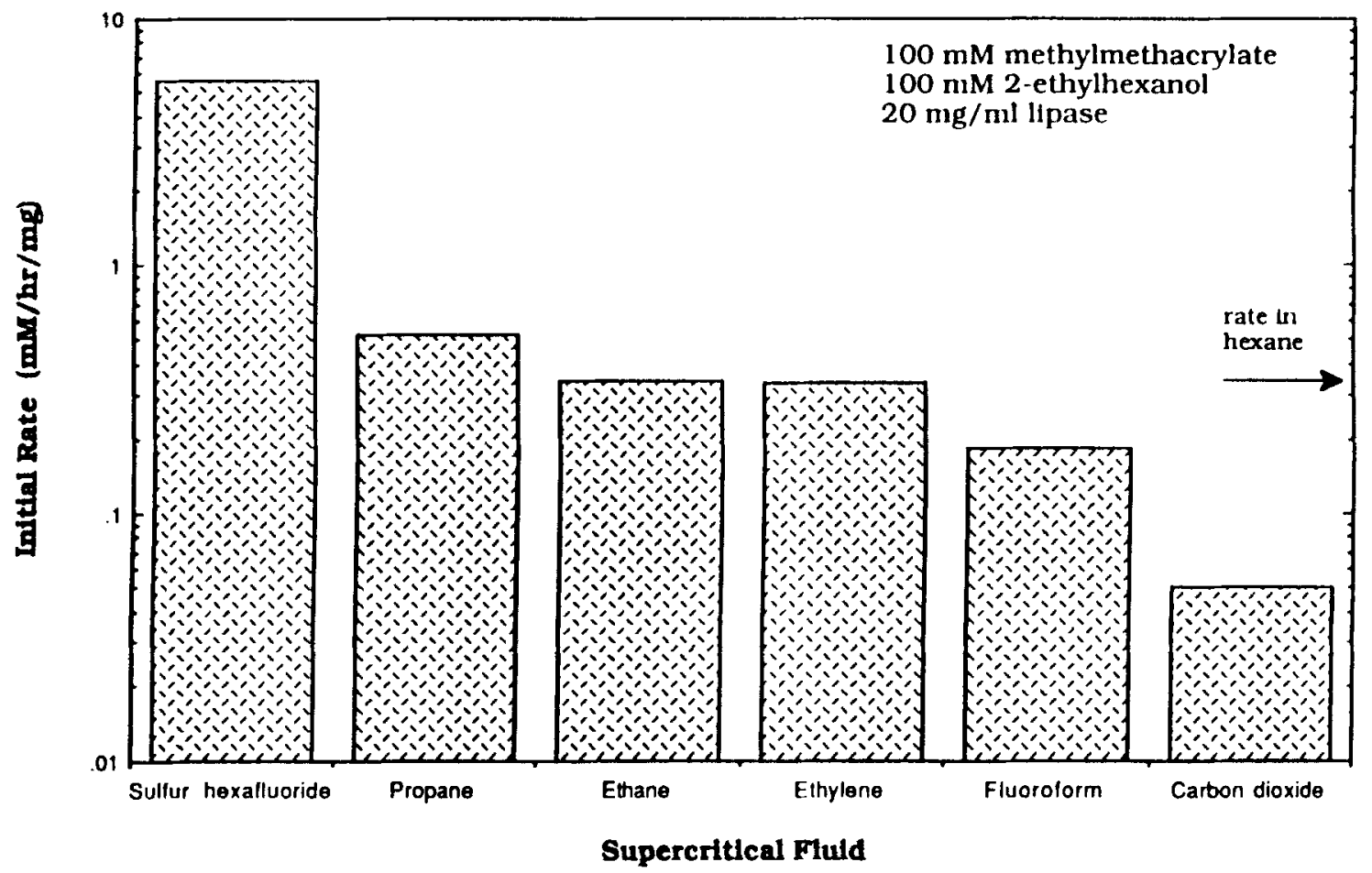

Figure 20. Rates of lipase-catalyzed reaction between 2-ethylhexanol and methylmethacrylate in SCFs at 1,600 psi (11 MPa) and $45^{\circ} \mathrm{C}$ (Kamat et al., 1992).

Courtesy of John Wiley \& Sons, Inc.

in cholesterol solubility in the fluid phase or to solvent-induced conformational changes of the enzyme. Using electron paramagnetic resonance (EPR) spectroscopy, they found evidence for the existence of cholesterol aggregates in the SCF. The presence of aggregates, or clusters, implied that the concentration of the reactant was not uniform throughout the SCF, but that regions of high local concentration existed. The ability of the different cosolvents to promote the formation of these aggregates correlated positively with the effect of the cosolvents on the reaction rate.

Ikushima et al. (1993) examined the effect of pressure on the lipase-catalyzed esterification of oleic acid and citronellol in $\mathrm{SC} \mathrm{CO}_{2}$. They found that the rate increased with increasing pressure, and that the rate correlated very well with the $E_{T}(30)$ value of the solvent, which provided a measure of the polarity of the solvent. They also observed that the stereoselectivity of the reaction varied with both pressure and temperature in $\mathrm{SC} \mathrm{CO}_{2}$, but not in hexane. They used Debenedetti's (1987) fluctuation theory to calculate the cluster size for the different operating conditions and found that the observed stereoselectivity correlated with the cluster size. Reaction conditions that led to the formation of large clusters also led to a high optical purity for the resulting product.

Work at the University of Pittsburgh (Kamat et al., 1992, 1993) has explored the use of compounds other than $\mathrm{CO}_{2}$ as SCF solvents for enzyme catalysis. The Pittsburgh group found that $\mathrm{SC} \mathrm{SF}_{6}$ gave the highest rates for the lipase-catalyzed transesterification of methylmethacrylate and 2ethoxyhexanol (Figure 20). The efficacy of $\mathrm{SF}_{6}$ was attributed to its high density and hydrophobicity compared to the other SCF solvents examined. Hydrophobic solvents appeared to be preferred for this synthesis because the water present in the reactor remains associated with the enzyme rather than being attracted to the SCF solvent phase. $\mathrm{SC} \mathrm{CO}_{2}$ was not a good solvent for this reaction, presumably because it altered the $\mathrm{pH}$ of the microaqueous enzyme environment or because it formed covalent complexes with free amine groups on the enzyme. Kamat et al. (1993) used these different SCFs to explore the effects of pressure-induced variations in solvent properties on the reaction rate. They found that when increasing pressure had an effect, it was to decrease the rate. They examined the influence of the density, solubility parameter, and dielectric constant on the rate and found that the dielectric constant was the parameter best suited for correlating the observed effects. A Kirkwood plot (Figure 21) for the reactions in SC fluoroform and ethane gave good linear correlations.

In closing this section on applications of SCFs in biochemical reactions, we note that processing in $\mathrm{SC}_{\mathrm{CO}}$ has been found to be a potentially attractive means of inactivating microorganisms in food and related materials (Lin et al., 1992; Kamihira et al., 1987a; Balaban et al., 1991; Castor and Hong, 1991). The SCF is thought to work by extracting essential intracellular components from within the cell and thereby rendering it inactive.

For additional details and information more specific to biocatalysis (e.g., enzyme stability, bioreactors, engineering issues) than to reactions in SCFs, we refer the interested reader to several recent summaries and reviews (Randolph and Carlier, 1992b; Randolph et al., 1991; Nakamura, 1990; Russell and Beckman, 1991a; Perrut, 1992). The minireview by Russell and Beckman (1991a) also includes a list of patents related to biocatalysis in SCFs.

Other Heterogeneous and Catalytic Reactions. Low (1985) 


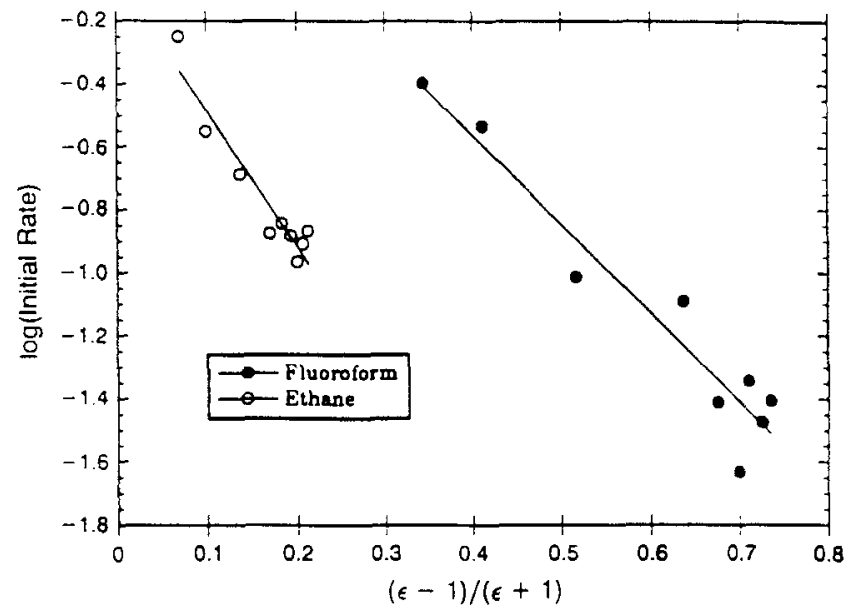

Figure 21. Kirkwood plot for lipase-catalyzed reaction in SC fluoroform (O) and SC ethane (O) (Kamat et al., 1993).

reported on the hydrotreating of heavy oils at supercritical conditions. He reasoned that there may be advantages to upgrading the supercritical extracts of oil shale or coal while the material was still at supercritical conditions. Low's experiments demonstrated the feasibility of supercritical upgrading for a number of different heavy hydrocarbon feeds. He found that an aliphatic solvent was better than an aromatic solvent because the aromatic solvent can be hydrogenated and consume expensive $\mathrm{H}_{2}$. He concluded that supercritical hydrotreating was superior to conventional liquid-phase hydrotreating because SC conditions led to a better product (less gas, more distillables) and less coke on the catalyst.

Gabitto et al. (1988) reported on the PbO-catalyzed dehydrogenation of toluene to dibenzyl and/or stilbene at supercritical conditions. The objective of their study was to determine whether this reaction would occur at supercritical conditions and then compare the results to those in the conventional gas-phase reaction. They found that at $4.76 \mathrm{MPa}$, dibenzyl was the major product at the supercritical temperature of $643 \mathrm{~K}$, but stilbene was the major product at the subcritical temperature of $453 \mathrm{~K}$. They also observed that the rate of dibenzyl production at $643 \mathrm{~K}$ increased as the pressure was increased from subcritical to supercritical values. The same reaction conducted in the gas phase at temperatures around $850 \mathrm{~K}$ and at atmospheric pressure produced primarily stilbene, with dibenzyl and benzene as byproducts. These results showed that the catalyzed reaction could occur at temperatures lower than those used previously provided the pressure was sufficiently high.

Herrick et al. (1988) reacted solid alumina with $\mathrm{CCl}_{4}$ at conditions that exceeded the critical point of the carbon tetrachloride. They examined this heterogeneous reaction, because it provides a potential route to $\mathrm{AlCl}_{3}$, which can be electrolytically converted to aluminum metal. The reaction is too slow to be commercially attractive when conducted at low pressures and $\mathrm{CCl}_{4}$ is in the gas phase. Operating at high pressures (4-10 MPa) and SC conditions, however, led to reaction rates that were orders of magnitude higher than those obtained with $\mathrm{CCl}_{4}$ in the gas phase. The authors attributed this enhanced reaction rate to a shift in the rate-determining step for the heterogeneous reaction. With $\mathrm{CCl}_{4}$ in the gas phase, the reaction is limited by the desorption of the $\mathrm{AlCl}_{3}$ product from the solid surface. With $\mathrm{CCl}_{4}$ present at higher densities and SC conditions, the desorption is much more rapid because $\mathrm{AlCl}_{3}$ is soluble in the SCF. Consequently, desorption is no longer rate limiting, and the much faster surface reaction becomes the rate-limiting step at SC conditions. The authors developed a quantitative model based on this mechanistic scenario and showed that it was consistent with their experimental results.

Collins et al. (1988) studied the disproportionation of toluene over a ZSM-5 zeolite catalyst at conditions near the critical point of toluene. This reaction is used commercially (at different operating conditions) to produce $p$-xylene. Byproducts include benzene and $o$ - and $m$-xylene isomers, which are undesired. Collins et al. speculated that if clustering occurred near the critical point, then the $p$-xylene that is formed could be hindered from undergoing secondary isomerization reactions. Hence, clustering might be a means to control selectivity in a reacting system. The experimental results, displayed in Figure 22, showed that the selectivity to $p$-xylene at $593 \mathrm{~K}\left(T_{r}=1.002\right)$ was highest near the critical pressure (4.1 $\mathrm{MPa}$ ), and it decreased at pressures that were either lower or higher. The authors concluded that their results were "not inconsistent with a mechanistic interpretation of near-critical clustering."

Suppes and McHugh (1989) studied the effects of different surfaces on the decomposition of cumene hydroperoxide in $\mathrm{SC}$ krypton, xenon, $\mathrm{CO}_{2}$, propane, and $\mathrm{CHF}_{2} \mathrm{Cl}$. They reported that the observed first-order rate constants were strong functions of the metals present during the reaction. Gold and 316 stainless-steel surfaces consistently gave larger rate constants than did Teflon-coated surfaces at otherwise identical conditions. The authors also observed that the different SCF solvents influenced the reaction rate. They could explain the solvent effect using transition-state theory only if they assumed that the activated complex was so short-lived that it could not interact with the solvent and thereby could not experience the different properties of the different SC solvents.

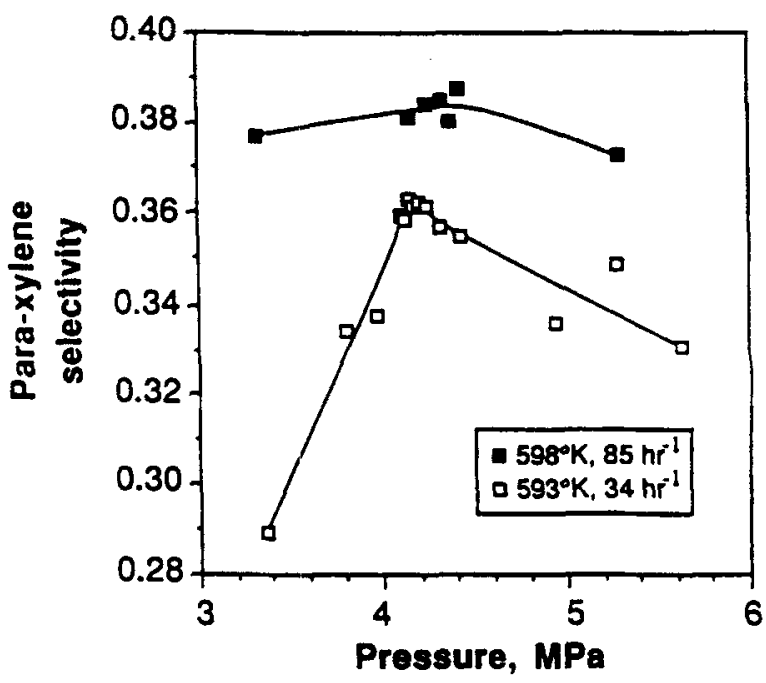

Figure 22. $p$-Xylene selectivity as a function of pressure at near-critical conditions (Collins et al., 1988). 
Vieville et al. (1993) accomplished the esterification of oleic acid with methanol in $\mathrm{SC} \mathrm{CO}_{2}$ using a cation exchange resin. They reported ester yields of about $50 \%$ for reactions at 313 $\mathrm{K}$ and 160 bars. They also found that the reaction rate was limited by transport of the reactants to the external surface of the resin.

Adschiri et al. (1991b, c) removed $70 \mathrm{wt}$. \% of the nitrogen from coal-derived pitches by catalytically hydrogenating the pitch in an SC toluene/tetralin mixture at $723 \mathrm{~K}$. These pitches could be used for the production of high-quality electrode carbon. The more conventional process for nitrogen removal involves catalytic hydrotreating of the pitch in the liquid phase. The higher diffusivities afforded through processing in the SCF resulted in higher reaction rates. Moreover, catalyst coking could be reduced significantly by increasing the pressure and hence the solvent power of the SCF.

\section{Fuels processing}

Using supercritical fluids as solvents or reactants in processing schemes to convert heavy hydrocarbons to liquid or gaseous fuels has been actively explored since at least 1970 . The work in this field evolved in parallel with the use of SCFs in biomass utilization (see section titled Conversion and Treatment of Biomass), and workers in both fields were addressing the same general types of problems (i.e., improving yields of liquid products, suppressing gas formation, and suppressing retrogressive reactions). In this section we present a brief summary of work with actual heavy hydrocarbon resources (oil shale, coal) and then a longer discussion of work with model compounds that has helped to reveal some of the fundamental chemistry.

Oil Shale. Conventional processes employed for the recovery of organics from oil shale involve pyrolysis and hydropyrolysis, at times in the presence of a catalyst. Coking, catalyst deactivation, and cracking reactions that lead to gases at the expense of oil and also result in high hydrogen consumption are among the problems encountered during conventional processing. To overcome these problems, processing schemes involving extraction and reaction in dense hydrogen-donor (such as tetralin), nonhydrogen donor (such as toluene), and inorganic (such as water) solvents have been explored (Funazukuri et al., 1988). At times, the reaction conditions exceeded the critical temperature and pressure of the solvent.

Tyler and Cane (1982) compared the pyrolysis of oil shale at high pressures in the presence and absence of supercritical steam. They found that the "wet"pyrolysis (pyrolysis in SCW) led to a lower degree of unsaturation in the pyrolyzate and a higher aromatic content than did the "dry" pyrolysis.

Baldwin and coworkers (Baldwin et al., 1986; Baldwin and Chen, 1987; Baldwin and Manley, 1988) found SC toluene and tetralin at $T=380-460^{\circ} \mathrm{C}$ and $P=15.1-20.6 \mathrm{MPa}$ to be excellent media for facilitating pyrolysis and hydropyrolysis of oil shale. Over $95 \%$ of the organic carbon could be converted to oil at short residence times and high temperatures. Hydropyrolysis in tetralin generally led to higher oil yields than did hydropyrolysis in toluene. Hydrogen donors can "cap" free radicals and thereby inhibit coke-forming condensation reactions and gas-forming cracking reactions. These effects lead to enhanced organic carbon conversions to oil. The extent of conversion to oil was remarkably insensitive to the nature of solvent employed provided that the solvent density was high and a hydrogen source was available. Given these restrictions, both subcritical and supercritical fluids performed equally well in promoting the conversion of oil shale to shale oil.

Yürüm et al. (1986) used FTIR and differential scanning calorimetry to monitor the progress of the reaction of oil shale in $\mathrm{SC}$ toluene. These investigators reported the presence of endothermal events at 209 and $304^{\circ} \mathrm{C}$, indicating that pyrolysis reactions occurred during the heat-up time to supercritical conditions. Thermal events were absent after supercritical conditions were achieved. They also reported that $48 \%$ of the organic material was extracted in the first $30 \mathrm{~min}$. After this initially rapid extraction, the rate of recovery of the remaining organic material was relatively slow.

Triday and Smith (1988) developed a mathematical model for the continuous extraction of kerogen from oil shale. The model accounted for reaction, pore diffusion, interphase heat and mass transport, and convective flow through the reactor. The model satisfactorily described experimental results from the extraction of kerogen from a Colorado oil shale using SC toluene. The structures of the components of SC toluene extracts from a Maoming oil shale have been invesetigated by Kuang-zong et al. (1984).

Coal. The literature dealing with the use of supercritical fluids in coal processing has been most recently reviewed by Kershaw (1989). Maddocks et al. (1979) and Ross (1984) give earlier reviews that focus on supercritical extraction of coal and coal liquefaction in $\mathrm{CO}$ /water systems, respectively. The articles we discuss here were either omitted from or published after Kershaw's review.

Most of the applications of SCFs in coal processing involve extraction and liquefaction. Using an SCF provides several advantages. Coal thermolysis in an SCF (rather than in an inert gas) provides improved solubility for the primary products, and can prevent cracking reactions to gases (Adschiri et al., 1991a) and/or condensation reactions to form char (Deshpande et al., 1984). An additional mechanism of action of an SCF is to penetrate the microporous structure of the coal and extract or react with material physically trapped within the micropores (Kershaw, 1989). Finally, SCF processes may also offer advantages for the downstream processing and separation of products. Gangoli and Thodos (1977) discuss these and other advantages that prompted much of the initial research in SCF processing of coal.

$\mathrm{SC}$ toluene and water are commonly used in coal processing because their critical temperatures fall within the $300^{\circ} \mathrm{C}$ to $400^{\circ} \mathrm{C}$ range, which corresponds to typical liquefaction conditions, and because they are stable under these conditions. It should be noted, however, that these $\mathrm{SC}$ solvents are not unreactive under these conditions. Toluene, for example, can react to form substituted biphenyls under these conditions (Ceylan and Olcay, 1981). Tetralin, a good hydrogen donor, has also been employed as an SC medium for coal liquefaction. Mixtures of these solvents have also been explored (Adschiri et al., 1991a). There have also been reports of coal extraction using SC alcohols (Yürüm et al., 1993; Kuznetsov et al., 1990; Makabe and Ouchi, 1981) and dynamic kinetic models have been developed for these extractions (Wang et al., 1993, 1994; Zhang et al., 1993). Figure 23 


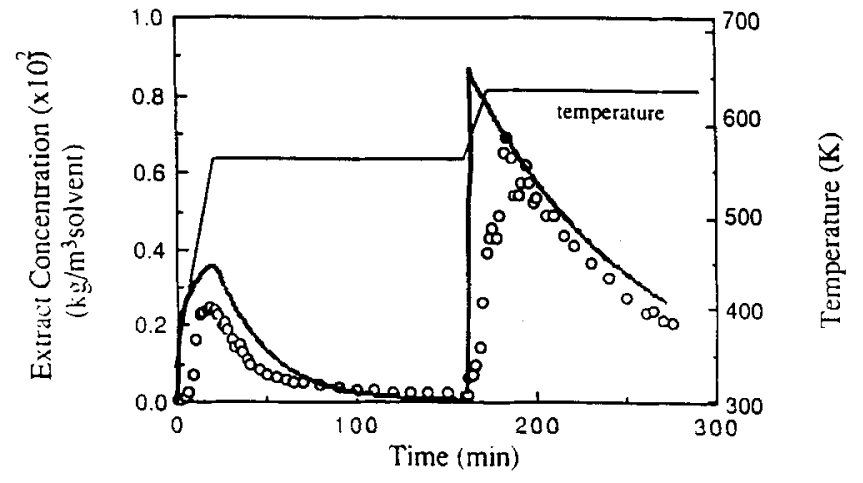

Figure 23. Comparison of model calculations and experimental data (open circles) for temperature-programmed coal extraction in SC $t$-butanol (Zhang et al., 1993).

Courtesy of American Chemical Society.

displays the ability of one of these dynamic models to capture the key features of the extraction process during a temperature-programmed experiment.

Deshpande et al. (1984) investigated the reactive extraction of hydrocarbons from coal using SC water in a batch autoclave, which permitted rapid injection of the coal sample into the SCF medium. They found that the amount of material extracted increased with the SCF density. For instance, increasing the density from 0.16 to $0.30 \mathrm{~g} / \mathrm{cm}^{3}$ increased the yield of tetrahydrofuran- (THF) soluble products from 40 to $75 \mathrm{wt} \%$. They also reported that the time-temperature history of the coal as determined by the heating protocol was important. For instance, at $375^{\circ} \mathrm{C}, 0.3 \mathrm{~g} / \mathrm{cm}^{3}$ water density, and a 15 min residence time, introducing the coal directly into SC water produced $75 \%$ THF solubles, whereas mixing water and coal at ambient conditions and then heating them together to supercritical conditions produced only $60 \%$ THF solubles. Similar trends for the variation in the yield of THF solubles with solvent density were also reported when SC toluene was the solvent (Deshpande et al., 1986). This group formulated a kinetic model based on the hypothesis that a part of coal dissolved instantaneously in the SCF and underwent liquefaction reactions that formed oils, asphaltenes, gases, and char. The model correlated the experimental results and was consistent with the important observable trends.

Sunol and Beyer (1990) performed a set of well-designed experiments to investigate the effects of time, temperature, flow rate, and pressure on the SC toluene reactive extraction of volatiles from coal. They found that temperature and pres. sure were the key variables and that their effects were interactive and not independent. These investigators also proposed a reaction network for the reactive extraction of coal. Ceylan and Olcay (1981) had shown earlier that the yield of material extracted from coal with SC toluene increased with pressure. They reported a $19 \%$ yield at $360^{\circ} \mathrm{C}$ and $11.6 \mathrm{MPa}$, and the yield increased to $32 \%$ at $29 \mathrm{MPa}$. The chemical nature of the compounds extracted into SC toluene have also been examined (Bartle et al., 1975, 1979; Tugrul and Olcay, 1978; Martin and Williams, 1981).

The removal of heteroatoms from coal and coal-derived products is another aspect of coal processing in which SCFs have been employed (Yürüm et al., 1993; Li and Egiebor,
1992). Muchmore et al. (1986), for example, used SC ethanol to remove organic sulfur from coal. They desired to make the coal suitable for use as a fuel in existing coal-fired utility boilers. Cahill et al. (1989) reported that $S$-containing fragments were easier to extract in $\mathrm{SC}$ toluene than were $O$-containing fragments. Li and Egiebor (1992) compared the efficacy of SC water and toluene for oxygen removal from coal. They found that $\mathrm{SC}$ water was a better medium for both oxygen removal and coal conversion. They suggested that the superiority of water could be attributed to its ability to engender bond cleavage through hydrolysis reactions.

Amestica and Wolf $(1983,1985)$ studied the catalytic liquefaction of coal in SC toluene, ethanol, and tetralin. They found that both toluene and ethanol gave low coal conversions $(<35 \%)$ and low liquid yields, whereas tetralin gave conversions around $90 \%$. Moreover, the results did not appear to be sensitive to whether tetralin was above or below its critical point during the reactive extraction. That the SC solvents have hydrogen donor capabilities appeared to be the controlling factor. Adding a CoMo hydrotreating catalyst to the system during the extractions with tetralin had only a small effect on coal conversion. The main effect was to enhance hydrotreating reactions and produce lighter products. They found that operating above the critical point increased the extent of hydrotreating reactions leading to lighter products, but had no significant effect on coal conversion. The main advantage of the SCF appeared to be its provision of a higher diffusivity, and hence diffusion rate in and out of the catalyst pores.

A number of investigators have considered the liquefaction of coal in a $\mathrm{CO}$ and SC water medium. It has been wellestablished that this medium is effective for liquefaction and that the effectiveness is not due to the in-situ generation of $\mathrm{H}_{2}$ via the water gas shift reaction. Ross (1984) gives a good introduction to and overview of this field.

Amestica and Wolf (1986) investigated the effect of catalysts and organic solvents on the liquefaction of coal in $\mathrm{CO} / \mathrm{H}_{2} \mathrm{O}$ systems. They found that high coal conversions could be obtained in supercritical $\mathrm{CO} / \mathrm{H}_{2} \mathrm{O}$ /solvent systems with or without an added catalyst. For example, at one set of conditions they reported that SC water alone led to $25 \%$ coal conversion and $11 \%$ desulfurization. Adding $\mathrm{CO}$ increased the coal conversion to $33 \%$ and the desulfurization to $20 \%$. Adding an organic solvent (tetrahydroquinoline was the most effective) increased coal conversion further, but adding a CoMo catalyst gave only very slight incremental improvements. The authors suggested that incorporating tetrahydroquinoline as a solvent led to a significant increase in coal conversion because it could disperse and dissolve the coal fragments.

Ross et al. $(1983,1985,1987)$ studied coal liquefaction using $\mathrm{CO}$ in $\mathrm{SC}$ water $\left(\mathrm{H}_{2} \mathrm{O}\right.$ or $\left.\mathrm{D}_{2} \mathrm{O}\right)$. As shown in Figure 24, liquefaction in $\mathrm{D}_{2} \mathrm{O}$ gave a higher conversion to toluene-soluble products than did liquefaction in $\mathrm{H}_{2} \mathrm{O}$. The authors used this result to dispute the notion that coal liquefaction was intrinsically limited by coal structure and that conversions higher than about $50 \%$ could be obtained only at the expense of product quality. Ross et al. proposed that the $\mathrm{CO}$ reacted with $\mathrm{OH}^{-}$to produce formate ion as an intermediate. This intermediate could react with water to form formic acid, which rapidly decomposed to form $\mathrm{CO}_{2}$ and $\mathrm{H}_{2}$, or react 


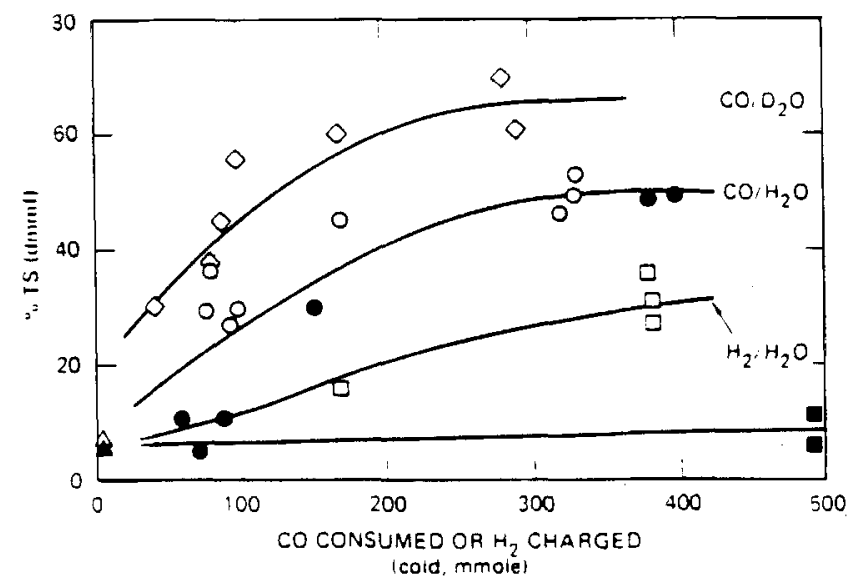

Figure 24. Coal conversion to toluene-solubles at $400^{\circ} \mathrm{C}$ for a 20-min reaction in different reaction environments.

Filled symbols are results from a demineralized coal (Ross et al., 1987).

Courtesy of American Chemical Society.

with coal and form toluene-soluble coal conversion products. Coal conversion was higher in $\mathrm{D}_{2} \mathrm{O}$ than in $\mathrm{H}_{2} \mathrm{O}$ because the formic acid formation reaction was slowed (by the kinetic isotope effect), and consequently the steady-state concentration of formate ion was higher in $\mathrm{D}_{2} \mathrm{O}$ than in $\mathrm{H}_{2} \mathrm{O}$. This higher formate concentration led to higher coal conversions. Mikita and Fish (1986) preferred a free-radical hydrogenolysis mechanistic model for coal liquefaction in SC water since their isotope experiments with coal did not show significant isotope effects. Further, the exclusion of molecular hydrogen from the system led to considerably lower coal conversions, which the authors cited as support for a free-radical mechanism. The authors did note, however, that some ionic pathways, particularly an electrophilic aromatic substitution reaction, may be operative during coal liquefaction in $\mathrm{SC}$ water.

Penninger $(1985,1989)$ examined the reactive extraction of oil from coal with aqueous solvents and found that the $\mathrm{CO} / \mathrm{H}_{2} \mathrm{O}$ binary gave oil yields higher than $\mathrm{H}_{2} / \mathrm{H}_{2} \mathrm{O}$, $\mathrm{N}_{2} / \mathrm{H}_{2} \mathrm{O}$, and $\mathrm{CO}_{2} / \mathrm{H}_{2} \mathrm{O}$. He also reported that the oils from aqueous-phase extraction had a lower oxygen content and higher carbon content than did oils from pyrolysis. Penninger postulated that the mechanism for the enhancement of liquefaction upon addition of $\mathrm{CO}$ was the influence of $\mathrm{CO}-\mathrm{H}_{2} \mathrm{O}$ chemistry (formate ion formation) on the ionic strength of the solution. An increased ionic strength enhanced the rates of the aqueous hydrolysis reactions that occurred under these conditions. This was a manifestation of the secondary salt effect. Sweeny et al. (1989) showed that temperature-programmed liquefaction to supercritical conditions gave higher product yields than did liquefaction at a single isothermal temperature.

Some other applications of SCFs in coal processing have also been reported. Nywlt and Peter (1992) employed potassium salts dissolved in water at supercritical conditions to gasify coal. The phase separation that resulted brought about a higher concentration of the alkali catalyst in the liquid phase, causing a considerable promotion of the reaction as compared to carrying out the reaction in a catalyst-impregnated coke. Warzinski et al. (1992) used $\mathrm{SC} \mathrm{CO}_{2}$ to dissolve, transport, and disperse $\mathrm{Mo}(\mathrm{CO})_{6}$, a catalyst precursor, into coal particles. They noted that a SCF with high density and low surface tension could be a good medium for this task. The coal liquefaction itself did not involve supercritical fluids.

Coal model compounds. The complex structure and composition of hydrocarbon fuel sources like coal and oil shale frequently obscure the fundamental chemical reaction processes that occur during the processing of these materials in SCFs. Working with simple, well-defined model compounds that mimic the functional groups in coal offers a way to overcome the difficulties of working with coal itself. Of course, the price one may potentially pay for this more fundamental information is relevance. Relating the model-compound-deduced fundamentals to real SCF processes for coal becomes a key issue.

This section reviews work on the reactions of coal model compounds in and with SCFs. Because coal and biomass are related, some compounds investigated can serve as models of structures in both materials. Thus, we refer the interested reader to the section titled Conversion and Treatment of Biomass, which discusses SCF applications with biomass, for a review of additional work with model compound reactions in SCFs.

Klein's group at the University of Delaware studied the reaction pathways, kinetics, and mechanisms of solvolysis and pyrolysis of coal model compounds in SC water and other SCFs (Lawson and Klein, 1985; Townsend and Klein, 1985; Abraham and Klein, 1985, 1987; Townsend et al., 1988; Abraham et al., 1988; Huppert et al., 1989; Wu et al., 1989, 1991a, b, c; Klein et al., 1990, 1992; Torry et al., 1992; Boock et al., 1992). They examined the effects of temperature, solvent density, phase behavior, and added salts, and used transition-state theory to interpret their results. Many of the model compounds were chosen to mimic the thermally labile aliphatic and heteroatomic bridges between aromatic clusters in coal. These compounds had the general structure $\mathrm{PhCH}_{2} \mathrm{XPh}\left(\mathrm{X}=\mathrm{CH}_{2}, \mathrm{O}, \mathrm{NH}\right.$, or S) or $\mathrm{PhCH}_{2} \mathrm{XYPh}(\mathrm{XY}=$ $\mathrm{CH}_{2} \mathrm{CH}_{2}, \mathrm{OCH}_{2}$ or $\mathrm{CH}_{2} \mathrm{O}$ ), where $\mathrm{Ph}=\mathrm{C}_{6} \mathrm{H}_{5}$.

The reactivity of the compounds these investigators studied led to their classification into one of three groups. Some compounds (e.g., dibenzyl ether, phenethyl phenyl ether, guaiacol, benzyl phenyl ether, and benzylphenylamine) reacted through parallel pyrolysis and solvolysis pathways, with the selectivity to the latter pathway being influenced by the density of the SC solvent. For example, dibenzyl ether gave toluene and benzaldehyde as the major neat-pyrolysis products, whereas pyrolysis in SC water at $T=383-400^{\circ} \mathrm{C}$ gave benzyl alcohol, via hydrolysis, as the major product (Townsend and Klein, 1985; Townsend et al., 1988). Other compounds (such as 1,2-diphenylethane and 1,3-diphenylpropane) reacted exclusively through pyrolysis reactions that were unaffected by SC water. The final compound class (e.g., phenyl ether, dibenzofuran) was unreactive under both neat pyrolysis and pyrolysis in SC water conditions. Based on these classifications, the authors determined that solvolysis occurred at a saturated carbon attached to a heteroatom-containing leaving group. These workers also showed (see Figure 25) that the hydrolysis rate constant increased with the dielectric constant of water, which was altered by changing the water loading. The variation of the rate constant with the 


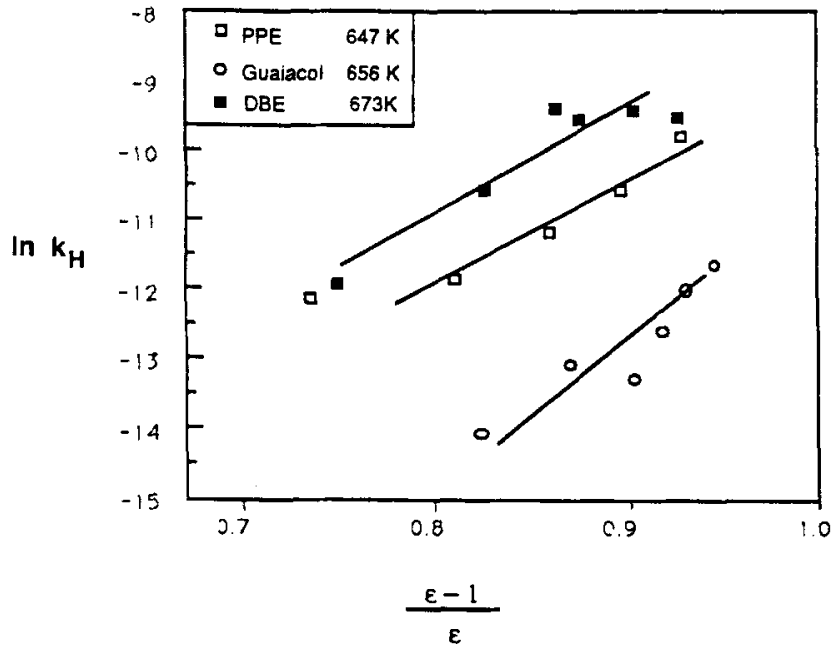

Figure 25. Kirkwood plot for the hydrolysis rate constant for phenethyl phenyl ether (PPE), gualacol, and dibenzyl ether (DBE) in SC water (Klein et al., 1990).

Courtesy of PRA Press.

dielectric constant was consistent with Kirkwood theory (Eq. 17). The authors concluded that the dielectric constant influenced the reaction because the transition state was more polar than the reactants. Thus, media with high dielectric constants would stabilize the formation of the transition state, and hence enhance the reaction rate.

The water loading in the batch microreactors was an independent variable in Klein's experiments. Unfortunately, both the water density (and hence density-dependent solvent properties) and pressure are controlled by the water loading so these two important variables could not typically be varied independently. One way to vary the properties of SC water independent of the reaction pressure, however, is to add a salt to the SC water. Huppert et al. (1989) used this approach to separate electrostatic and hydrostatic effects on the hydrolysis of guaiacol in SC water. They found that the hydroly-

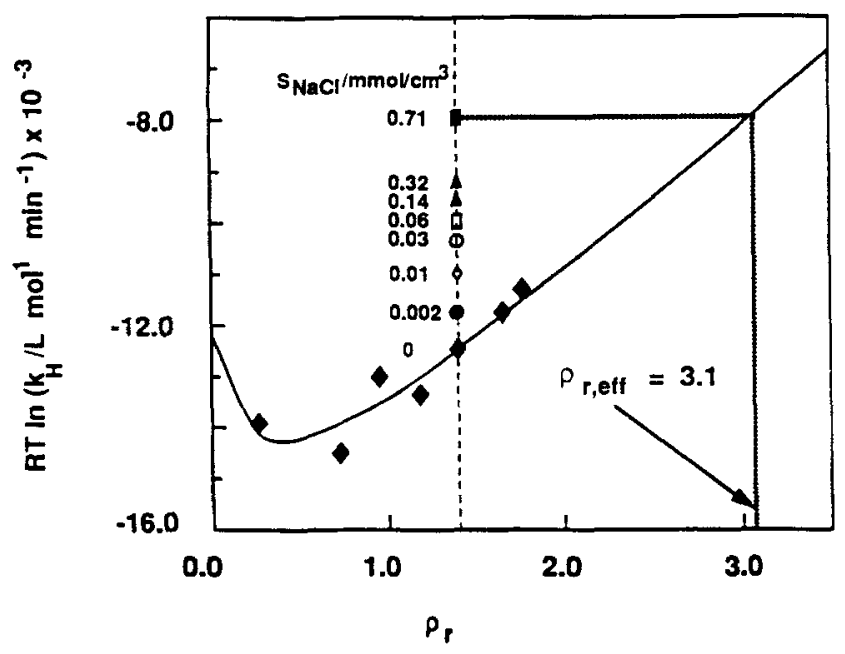

Figure 26. Effect of added salts and water density on guaiacol hydrolysis kinetics (Klein et al., 1990).

Courtesy of PRA Press. sis rate constant increased with the salt loading at a constant water density, as shown in Figure 26. The hydrolysis rate constant at a $\mathrm{NaCl}$ loading of $0.71 \mathrm{mmol} / \mathrm{cm}^{3}$ and a water density of $0.5 \mathrm{~g} / \mathrm{cm}^{3}$ was equivalent to that which might have been obtained in the absence of added salts, but at a water density of $1.1 \mathrm{~g} / \mathrm{cm}^{3}$. Thus, adding a salt is a means of increasing the "effective" density of SC water. A modified Hebrandson and Neufeld analysis (Eq. 15) revealed that the transition state for guaiacol hydrolysis was more polar than the reactants and products. In a subsequent study, Torry et al. (1992) found that the hydrolysis rate constant for dibenzyl ether and benzylphenyl amine initially increased with added salt, but subsequently decreased with higher salt loadings. This behavior was consistent with the added salt increasing the dielectric constant of the medium and hence the rate constant for hydrolysis, but also decreasing the number of water molecules available for hydrolysis by requiring water molecules to be solvated. These competing effects produced the maximum rate observed. A microscopic model based on these arguments was developed and shown to be qualitatively consistent with the experimental results.

Klein et al. (1990) later postulated an $S_{N^{2}}$ mechanism for the hydrolysis reaction, with water serving as the nucleophile. An $S_{N^{1}}$ mechanism was considered unlikely because the hydrolysis rate depended on the water concentration, because $\mathrm{Cl}^{-}$(from added $\mathrm{NaCl}$ ) did not add to the substrate, and because facile hydrolysis occurred even at low water densities where $K_{w} \sim 10^{-40}$. The excellent correlation of the Kirkwood analysis, the salt effect, and the hydrolysis rate not correlating with $K_{w}$ at low density were all cited as evidence supporting an $S_{N^{2}}$ mechanism with water as the nucleophile.

Wu et al. (1991b) used transition-state theory to interpret the effect of pressure on the reaction rate constant in the form of an apparent activation volume that could be decomposed into contributions from mechanical pressure effects, compressibility effects, electrostatic interactions, diffusion limitations, and phase behavior effects. Contributions for the latter three effects were evaluated. These investigators also examined the influence of density on reactions in SCFs (Wu et al., 1991c). They showed that the conversion of benzyl phenyl ether decreased with increasing solvent (toluene) density, as shown in Figure 27. They explained this phenomenon by noting that diffusion limitations, as described through cage-effect theory, caused a decrease in the initiation rate for benzyl phenyl ether as the solvent density increased.

Klein et al. (1992) probed solvent and substituent effects in the hydrolysis of substituted anisoles and substituted phenols. A classic Hammett formulation failed to correlate substituent effects on the rate constant, presumably because the SCF solvent effect and the substituent effect were coupled. To decouple these two effects, the authors extrapolated the measured hydrolysis rate constants to low water densities where solvent effects would presumably be negligible. The Hammett plot based on these extrapolated rate constants provided an excellent correlation. The Hammett reaction constant obtained from the plot increased as the water concentration decreased. Since the signs of the reaction constant for hydrolysis and the reported reaction constant for pyrolysis were opposite, it was conjectured that a basis for the design of a substituent-specific solvent effect for selective hydrolysis or pyrolysis may have been obtained. It must be noted, 


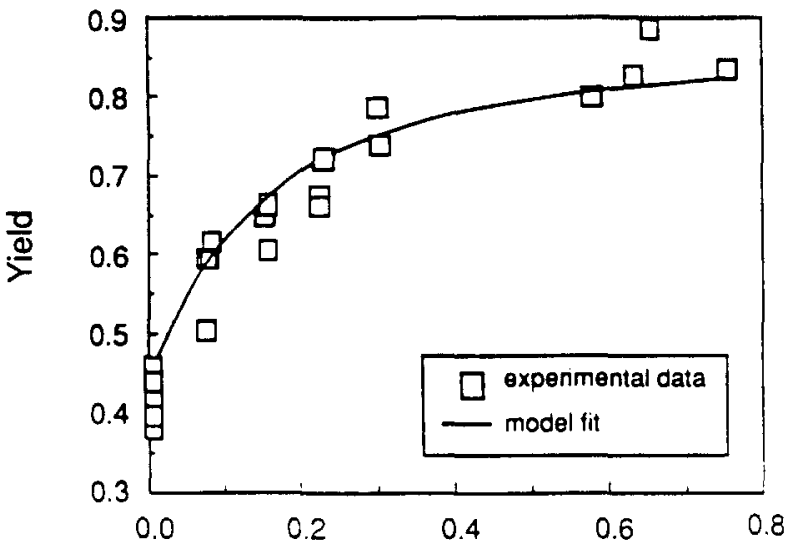

Toluene Density $\left(\mathrm{g} / \mathrm{cm}^{3}\right)$

Figure 27. Effect of SC toluene density on benzyl phenyl ether yield at $320^{\circ} \mathrm{C}$ (yield $=1$-conversion) (Wu et al., 1991b).

Courtesy of American Chemical Society.

though, that the inference was based on the behavior of the Hammett reaction constant with density when the reaction constants themselves were obtained for the limiting case of low density.

Houser's group at Western Michigan University has also examined the reactions of coal model compounds in SC water. Their research program focused on the removal of heteroatoms, especially nitrogen, from model compounds thought to be representative of structures found in coal. Compounds investigated include quinoline and isoquinoline (Houser et al., 1986; Li and Houser, 1992); tetrahydroquinoline, benzylamine and bibenzyl (Houser et al., 1989); benzaldehyde, benzylidenebenzylamine, benzyl alcohol, and benzoic acid (Tsao et al., 1992); and 1-chlorohexane, ethyl benzilate, trihexylamine, quinuclidine, phenylpiperidine, and phenylpyridine (Houser et al., 1993). Several of the compounds in this most recent report mimic structures in chemical weapons rather than coal. These investigators typically explored the effects of reaction time, water loading, and additives $\left(\mathrm{ZnCl}_{2}\right.$, dihydroanthracene, ammonia) on the kinetics and product yields.

The presence of $\mathrm{SC}$ water had a strong influence on the kinetics and product distributions for many, but not all, of the compounds. For example, reaction of isoquinoline at $450^{\circ} \mathrm{C}$ for 48 hours in the absence of water led to $<10 \%$ conversion, whereas reactions in SCW at densities of 0.106 and $0.213 \mathrm{~g} / \mathrm{cm}^{3}$ gave conversions of 55 and $81 \%$, respectively. Moreover, water participated in the reaction as evidenced by the production of benzyl alcohol and benzaldehyde from the reaction of benzylamine in SC water. The presence of SC water also reduced the formation of high molecular weight products from benzylamine pyrolysis. Bibenzyl, on the other hand, reacted at about the same rate in the presence and absence of SC water. The products were also very similar. Houser et al. (1989) noted that water was clearly a reactant in benzylamine pyrolysis but not in bibenzyl pyrolysis. They recognized that a $\mathrm{C}-\mathrm{N}$ bond was the distinguishing feature that facilitated hydrolysis (Tsao et al., 1992).
These results were consistent with and, at times, obtained in parallel with those of Klein's group.

Most of the nitrogen removed from the organic compounds appeared as ammonia in the aqueous phase. The addition of $\mathrm{ZnCl}_{2}$, an acid catalyst, increased the reactant conversion and the ammonia yields. The absence of pyridine or substituted pyridine in the quinoline decomposition products implied that the heterocyclic ring was more reactive than the homocyclic ring. The nitrile structure was thought to hydrolyze and decarboxylate to form hydrocarbons and ammonia (Houser et al., 1986).

$\mathrm{Li}$ and Houser (1992) determined the kinetics for the reaction of quinoline in $\mathrm{SC}$ water in the presence of a $\mathrm{ZnCl}_{2}$ catalyst. They found the reaction to be first order in both quinoline and catalyst and inverse first order in water in the temperature range of $400-500^{\circ} \mathrm{C}$. This rate law was consistent with a Langmuir-Hinshelwood mechanism with competitive adsorption between quinoline and water. The apparent activation energy being $112 \mathrm{~kJ} / \mathrm{mol}$, which is well below the dissociation energy of the $\mathrm{C}-\mathrm{N}$ bond in quinoline, was taken as evidence of a catalytic reaction mechanism.

Penninger (1988) studied the thermal decomposition of di$n$-butylphthalate (DBP), a model compound that mimicked ester functionalities thought to be important in a Wyoming coal, in near-critical and SC water. During neat pyrolysis, the butyl groups appeared primarily as butenes, but in the presence of water these alkyl fragments were recovered primarily as butanols. The presence of water in the reactor also suppressed the decarboxylation of benzoic acid, a secondary product of DBP decomposition, which under pyrolysis conditions would yield benzene and $\mathrm{CO}_{2}$. The $\mathrm{CO}_{2}$ group was thus retained in the liquid products ("oils") rather than adding to the gas make. Polycondensation of phenyl species was completely suppressed in SC water. Penninger noted that the long heat-up time in his reactor and experimental protocol made it difficult to determine the phase(s) present throughout this nonisothermal reaction.

Horiuchi et al. (1988) studied the thermolysis reactions of a series of substituted bibenzyls and $o$-hydroxybibenzyls in SC $\mathrm{H}_{2} \mathrm{O}$ and $\mathrm{D}_{2} \mathrm{O}$. Electron-donating substituents on the bibenzyls enhanced deuterium incorporation into the recovered starting materials. An electrophilic aromatic substitution mechanism was proposed to explain this finding. Further, the reaction of hydroquinone monobenzyl ether (HQMBE) in $\mathrm{D}_{2} \mathrm{O}$ yielded catechol monobenzyl ether (CMBE) as a product, with one deuterium substitution in the recovered HQMBE and three in the CMBE product. The authors noted that this observation was consistent with two successive nucleophilic aromatic substitutions of $\mathrm{D}_{2} \mathrm{O}$ with HQMBE. Thus, supercritical water could offer a medium for both electrophilic and nucleophilic substitution reactions.

In summary, research with coal model compounds in SCFs has revealed several important kinetic and mechanistic facets of coal chemistry. The SCF (especially SC water) is not necessarily an inert solvent, but rather an active participant in the chemical reactions of some compounds. At reaction conditions typically employed in these experiments, the SCF is both hot and dense, so reaction with the SCF can occur. Indeed, hydrolysis of coal model compounds in SC water increased with both temperature and the water density. Finally, these studies showed that some of the theories 


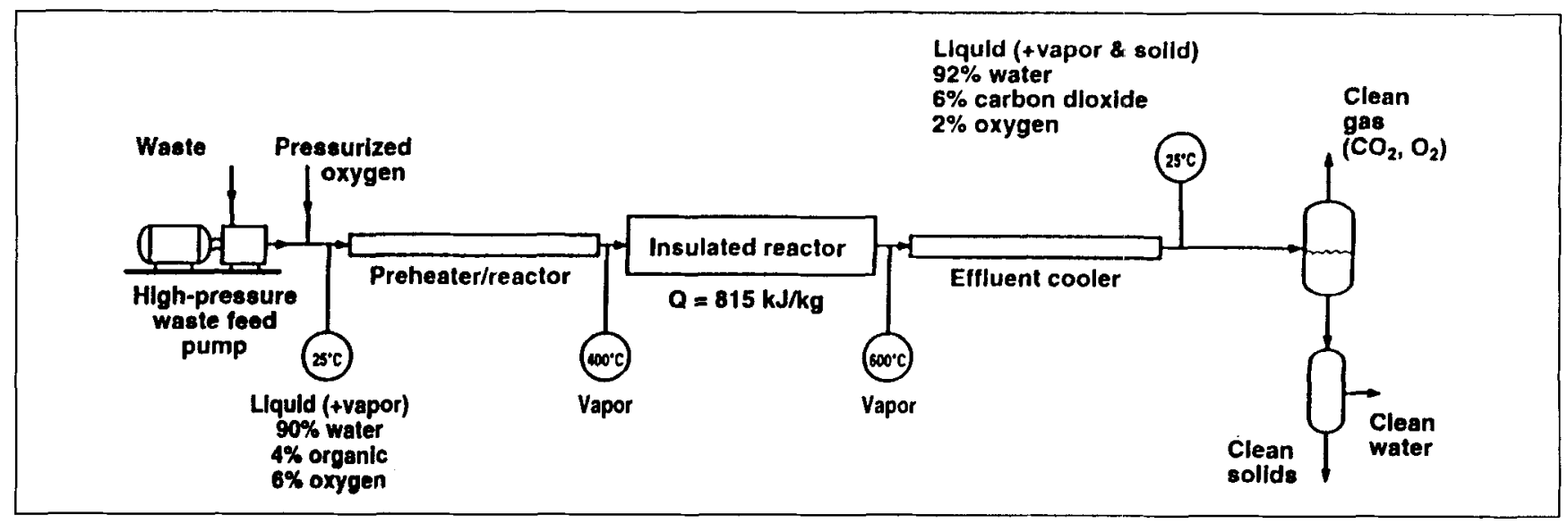

Figure 28. Simplified flowsheet for a SCWO process with representative compositions and temperatures (Modell et al., 1992).

and techniques (Kirkwood analysis, activation volumes, Herbrandson-Neufeld analysis, salt effects) used to study reactions in solution can also be used profitably to study reactions in SCFs.

\section{Oxidation reactions}

Oxidation chemistry in SCFs has received much attention. Most of this work has focused on supercritical water oxidation (SCWO), a waste treatment technology. Work with SCWO led to the identification and investigation of hydrothermal flames. There has also been some work on using SCFs as reaction media for synthesis via partial oxidation. Finally, a more established area of oxidation at supercritical conditions involves high-pressure, supercritical combustion of fuels. The last topic in this section briefly discusses the effect of autoxidation reactions on the stability of fuels at supercritical conditions.

Oxidation in supercritical water. The complete oxidation of organics in SC water is an effective means of treating many organic wastes including pulp and paper mill sludges (Modell et al., 1992), process wastewaters (Li et al., 1993a; Sawicki and Casas, 1993), hazardous organics (Modell, 1989; Staszak et al., 1987; Swallow and Killilea, 1992), sludges from water treatment plants (Shanableh and Gloyna, 1991), waste from manned space missions (Takahashi et al., 1989), biological wastes from the pharmaceutical industry (Johnston et al., 1988), mixed wastes (Dell'orco et al., 1993), and military wastes (Harradine et al., 1993; Rofer et al., 1992; Rice et al., 1993). SCWO converts hydrocarbons to carbon dioxide and water at reaction temperatures around 400 to $700^{\circ} \mathrm{C}$. Nitrogen is typically converted to $\mathrm{N}_{2}$ or $\mathrm{N}_{2} \mathrm{O}$. Unlike incineration, SCWO rarely produces $\mathrm{NO}_{x}$ or $\mathrm{SO}_{x}$ in detectable quantities because of the comparatively low temperatures used in SCWO (Thomason et al., 1990; Killilea et al., 1992; Timberlake et al., 1982). One exception is the report of high levels of $\mathrm{NO}_{x}$ in the effluent from the oxidation of TNT and other explosives with hydrogen peroxide (Harradine et al., 1993). Figure 28 gives a simplified process flowsheet for a SCWO process.

SCWO is most attractive economically for aqueous waste streams with organic concentrations around $1-20 \mathrm{wt}$ \%
(Thomason and Modell, 1984). If the organic concentration is too low, autothermal operation is not possible at supercritical temperatures, and an auxiliary fuel is required. If the organic concentration exceeds $20-25 \%$, on the other hand, incineration becomes competitive because the higher incineration temperatures $\left(1,000+{ }^{\circ} \mathrm{C}\right)$ can be maintained with minimal use of auxiliary fuel.

SCWO technology is an extension of wet-air oxidation, which operates at subcritical temperatures and pressures and was developed to treat organics in industrial wastewater streams. There are several advantages to conducting the oxidation reactions above, rather than below, the critical point. One advantage is that higher temperatures promote faster reaction rates. A second advantage is that a single fluid phase containing organics, an oxidant, and water can exist at reaction conditions. Many organic compounds are only sparingly soluble in water at ambient conditions. At supercritical conditions, however, nonpolar organics enjoy complete miscibility with the water. Wet-air oxidation, on the other hand, involves a two- or three-phase system, and mass transfer across the phase boundaries can limit the oxidation rates. A third advantage related to solubility is that salts have a reduced solubility in SC water. Therefore, salts formed from the neutralization of acids produced during SCWO can be precipitated from the solution. This is useful in purifying the reactor effluent stream (Thomason et al., 1990). One of the key drawbacks to SCWO technology is the expense of the reactor for this high-pressure process, which must be made of high nickel alloys such as Inconel 625 or Hastelloy C276 to withstand the potentially corrosive SCWO conditions. Even these materials show signs of degradation under the harshest of conditions (Barner et al., 1992; Bramlette et al., 1990).

Several SCWO reactors and process designs have been described (Modell, 1982, 1985, 1989; Modell et al., 1993; Hong, 1991; Barner et al., 1992; Huang, 1992; Huang et al., 1992; Titmas, 1986). Some of these reactors could be used as small laboratory disposal units, some as mobile units that could be transported to specific sites (Eckert et al., 1990; McFarland et al., 1991), and some as permanently based units that could generate power from the heat released by destroying the waste products. One type of permanent unit is the vertical deep-well reactor proposed for SCWO applications (Titmas, 


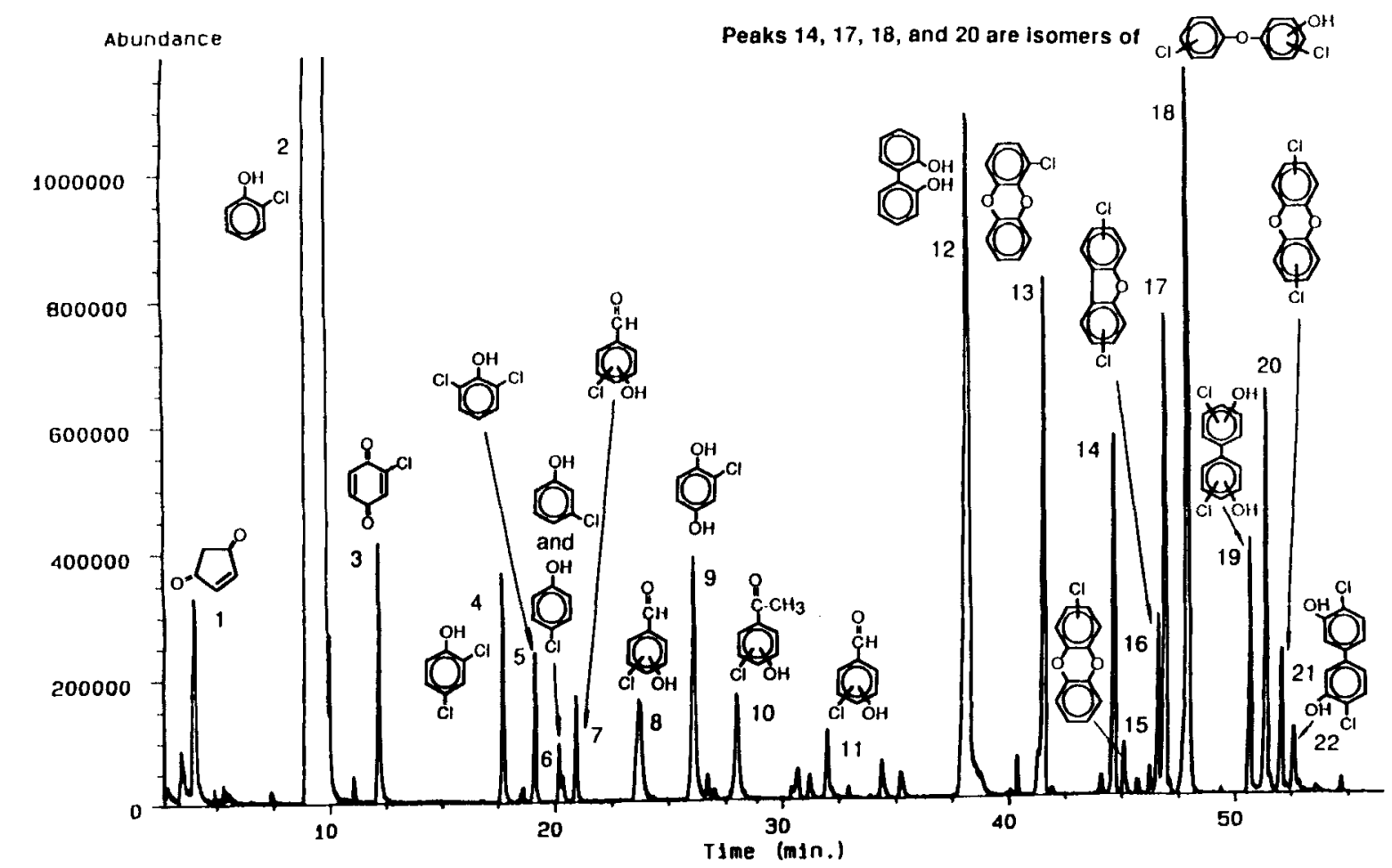

Figure 29. Total ion chromatogram of 2-chlorophenol oxidation products in SC water at $380^{\circ} \mathrm{C}, 278$ atm and $24.7 \mathrm{~s}$ residence time (Li et al., 1993).

1986). This reactor could descend a mile below the surface of the earth, and it achieves the high pressure required by taking advantage of the static head of the fluid. Kodra and Balakatoiah (1992) recently modeled this type of deep-well reactor.

Air or $\mathrm{O}_{2}$ are the most common oxidants used in SCWO, but $\mathrm{H}_{2} \mathrm{O}_{2}, \mathrm{KMnO}_{4}, \mathrm{Cu}-\mathrm{H}_{2} \mathrm{O}_{2}, \mathrm{Fe}-\mathrm{H}_{2} \mathrm{O}_{2}$, and $\mathrm{MnSO}_{4}-\mathrm{H}_{2} \mathrm{O}_{2}$ mixtures have also been explored as potential oxidants (Lee and Gloyna, 1992; Lee et al., 1990; Shanableh and Gloyna, 1991; Chang et al., 1993). Although these alternative oxidants are more costly than $\mathrm{O}_{2}$ or air, they may facilitate oxidation at lower temperatures, which could offset these higher costs.

The previous paragraphs gave but a brief overview of SCWO technology and some of the process engineering issues. We refer the reader interested in the engineering aspects of SCWO to several other discussions of SCWO process technology and process development (Modell, 1989; Tester et al., 1993; Thomason et al., 1990; Barner et al., 1992). The balance of this section will focus on the oxidation reactions in SC water, rather than the process of SCWO.

1. Products and pathways. Identifying and quantifying the yields of the intermediate oxidation products is an essential prerequisite for evaluating the potential environmental impacts of SCWO technology and for developing reaction networks for SCWO. The most extensive efforts devoted to the identification of intermediate oxidation products of representative pollutants has centered on phenol and substituted phenols. Yang and Eckert (1988), for example, identified $p$ benzoquinone as a major product from $p$-chloropheno oxidation in SC water. By operating a flow reactor at temperatures only slightly above the critical temperature and using short residence times, Savage and coworkers identified a large number of intermediate products from the oxidation of phenolics. Thornton and Savage (1990) identified 16 different products from phenol oxidation, and they reported the yields of some of the products. These products included carboxylic acids, dihydroxybenzenes, and phenol dimers (phenoxyphenols and biphenol). In subsequent studies (Thornton et al., 1991; Thornton and Savage, 1992a), this group expanded the list of identified products to include nearly $40 \mathrm{com}$ pounds, and they reported more extensive data for the yields of the phenol dimers and the related products dibenzofuran and dibenzo-p-dioxin. The products formed during 2-chlorophenol oxidation ( $\mathrm{Li}$ et al., 1993) were similar to those observed from phenol. With this compound, however, chlorinated dibenzofurans and chlorinated dibenzo- $p$-dioxins were produced, as shown in the total ion chromatogram in Figure 29 . Such compounds are potentially more hazardous than the 2-chlorophenol reactant. Recognizing the significance of dibenzo- $p$-dioxin and dibenzofuran formation during this waste treatment process, Thornton and Savage (1992a) sought the global reaction pathways for phenol oxidation that led to these products. They found that the phenol dimers were primary products of phenol oxidation, and they suggested that these dimers were the precursors to dioxin formation. Li et al. (1993) illustrated the key steps in the global reaction network, as shown in Figure 30. There were two parallel primary reactions. One led to dimers and dibenzofurans and dibenzodioxins. The second primary pathway led to substituted phenols and ring-opening products. Both of these groups of products were ultimately converted to $\mathrm{CO}_{2}$. For both phenol and chlorophenol, a significant fraction (about 50\%) of the phenolic compound reacted to form dimers.

Crain et al. (1993) identified nearly 20 intermediate prod- 


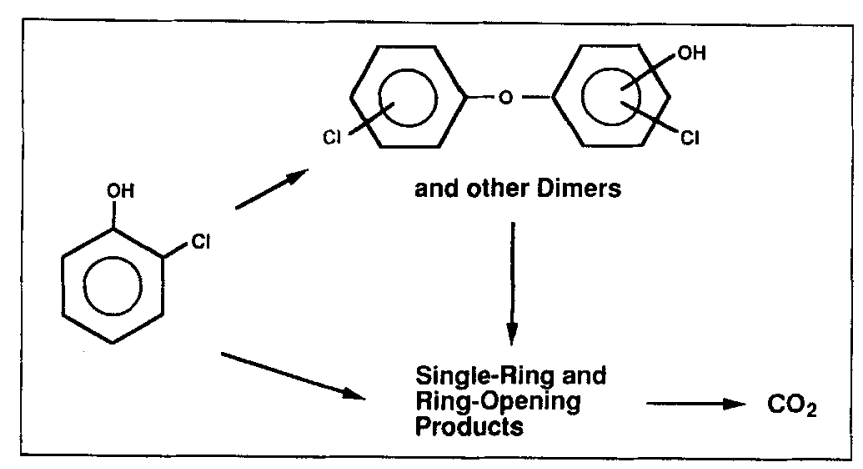

Figure 30. Global reaction network for 2-chlorophenol oxidation in SC water ( $\mathrm{Li}$ et al., 1993).

ucts from the oxidation of pyridine in SC water. These products included amines, amides, and carboxylic acids. No quantitative information about the product yields was given. The authors postulated a set of reaction pathways for the oxidation reaction, but they provided no quantitative assessment of the validity of these pathways.

Li et al. (1991, 1993b) have proposed generalized reaction networks for the oxidation of organics in near-critical and SC water. Their premise is that complex mixtures oxidize via two parallel reaction paths. One is direct oxidation to stable end products such as carbon dioxide and nitrogen. The second is initial oxidation to low molecular weight intermediates, which are then slowly oxidized to the end products. Acetic acid and ammonia have been advanced as key slowly oxidizing intermediates. Acetic acid was singled out as the key organic intermediate because it is a frequent product of wet-air oxidation. Further, kinetics studies showed that acetic acid oxidized more slowly than some other organic compounds (Lee et al., 1990). We note, however, that there are conflicting reports (Wightman, 1981; Rice et al., 1993) showing that the reactivity of acetic acid exceeds that of several other organics. Ammonia was taken to be the nitrogen-containing refractory intermediate because it is thought to be resistant to destruction at temperatures below $500^{\circ} \mathrm{C}$. A parameter $\alpha$ characterizes the "strength" of the waste. Values of $\alpha$ approaching unity imply that most of the oxidation proceeds through a refractory intermediate. By assuming first-order kinetics and adjusting the values of the parameters in this simple global scheme, one can correlate a large amount of diverse experimental data fairly well. Interestingly, however, none of the studies on the oxidation of phenolics in SC water has reported acetic acid as an intermediate product, yet the values of $\alpha$ reported for phenol and 2-chlorophenol are 0.15 and 0.37-0.96, respectively ( $\mathrm{Li}$ et al., 1991, 1993b). Additionally, Li et al, (1992) used the low activation energies they found for the kinetics of $\mathrm{CO}_{2}$ formation during SCWO of phenolics to question the role of a refractory intermediate as a general feature of SCWO reaction networks.

2. Global kinetics. There have been several kinetics studies of the SCWO of individual compounds (Helling and Tester, 1987, 1988; Webley and Tester, 1989, 1991; Yang and Eckert, 1988; Rofer and Streit, 1988, 1989; Thornton and Savage, 1990; Lee et al., 1990; Webley et al., 1990, 1991; Jin et al., 1990, 1992; Holgate and Tester, 1993; Holgate et al., 1992; Tester et al., 1992; Thornton and Savage, 1992b; Lee and Gloyna, 1992; Li et al., 1992, 1993; Crain et al., 1993; Chang et al., 1993; Savage et al., 1994a; Holgate and Tester, $1994 a, b)$. Most of these have focused on the kinetics of the disappearance of the organic compound, but some have also considered the kinetics of the conversion of organic carbon to $\mathrm{CO}_{2}$ ( $\mathrm{Li}$ et al., 1992). One of the common goals of these investigations was to summarize concisely all of the experimental data using an empirical power-law global rate expression. Such global rate laws can be used in the design, analysis, and control of SCWO reactors and processes. Tester et al. (1993) give a summary of SCWO rate laws and their parameters.

The reaction order for the organic compounds studied to date was nearly always close to unity. An exception is the report by Yang and Eckert (1988), which indicated a shift in the kinetics of 4-chlorophenol oxidation from being first order at low concentrations to being second order at high concentrations. Li et al. (1993), however, showed that Yang and Eckert's data were fully consistent with their rate law for 2chlorophenol oxidation, which had a constant organic order of 0.88 and a nonzero oxygen reaction order.

The reaction order for oxygen appears to be more variable. Values around 0.5 were reported for phenol (Thornton and Savage, 1992b), 2-chlorophenol (Li et al., 1993), CO (Holgate et al., 1992), and methane (Webley and Tester, 1991), whereas values of zero were reported for methanol (Tester et al., 1992), hydrogen (Holgate and Tester, 1993), and 4-chlorophenol (Yang and Eckert, 1988). We note, however, that a recent reexamination ( $\mathrm{Li}$ et al., 1993) of the data for 4-chlorophenol showed that an oxygen order near 0.5 might provide a better description of those data.

The literature gives only a few values for the global reaction order for water, the third compound initially present in SCWO reactions. Unlike the concentrations of an organic compound or an oxidant, the concentration of water cannot typically be manipulated independently of all other variables because water is about $99 \%$ of the reaction mixture in most kinetics studies. To effect a change in the water concentration one needs to alter the system temperature or pressure. Unfortunately, both of these parameters can also influence the reaction rate constant through the activation energy, the activation volume, and the concentrations of organic and oxygen. Thornton and Savage (1992b) and Li et al. (1993) chose to explore the effect of the water concentration (density) by manipulating pressure at a constant temperature of $380^{\circ} \mathrm{C}$, which is near the critical point. The reaction rate was clearly density (or pressure) dependent, and the rate increased with increasing density. They reported a global water order of 0.7 for phenol oxidation and 0.34 for 2-chlorophenol oxidation. Holgate and Tester (1994a) examined the effect of pressure on the kinetics of $\mathrm{H}_{2}$ and $\mathrm{CO}$ oxidation in SC water. Figure 31 gives some of their results at $550^{\circ} \mathrm{C}$ and $570^{\circ} \mathrm{C}$. At these high temperatures, the density varied less strongly with pressure than it did at $380^{\circ} \mathrm{C}$. Nevertheless, the results for $\mathrm{CO}$ and $\mathrm{H}_{2}$ were consistent with the earlier results for phenol and chlorophenol in that the rate constant increased with pressure and density. The apparent reaction order for water was 1.7 for CO oxidation. Holgate and Tester (1994a) explained the effect of pressure on the global kinetics by examining the effect of pressure on elementary unimolecular reactions. That reaction rates vary with water density could also 


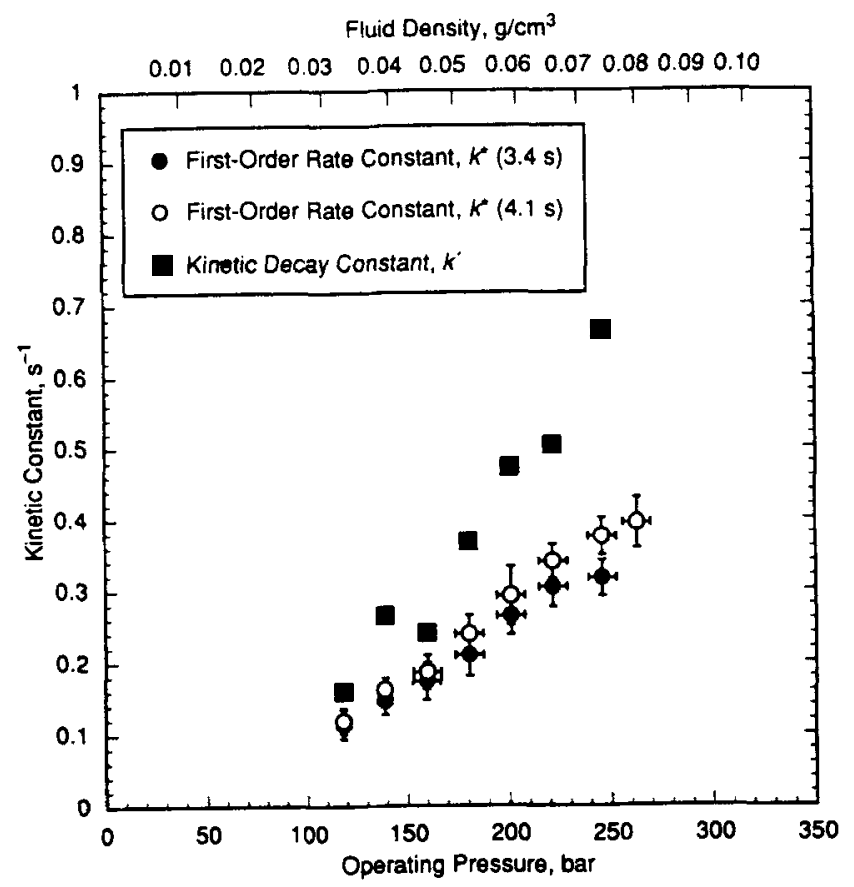

Figure 31. Effect of pressure (and water density) on CO oxidation kinetics in $\mathrm{SC}$ water at $570^{\circ} \mathrm{C}$ (Holgate and Tester, 1994a).

Courtesy of American Chemical Socicty.

be attributed to the variation of some density-dependent property, such as the dielectric constant, that can influence reaction rates. Savage et al. (1994a) recently showed (see Figure 32) that the effect of pressure on global rate constants for SCWO of phenols could be correlated with the dielectric constant.

That the organic reaction order is typically close to unity and that the oxygen order was reported as being near zero in early SCWO kinetics studies has led to these values sometimes being assumed for the analysis of data from new com-

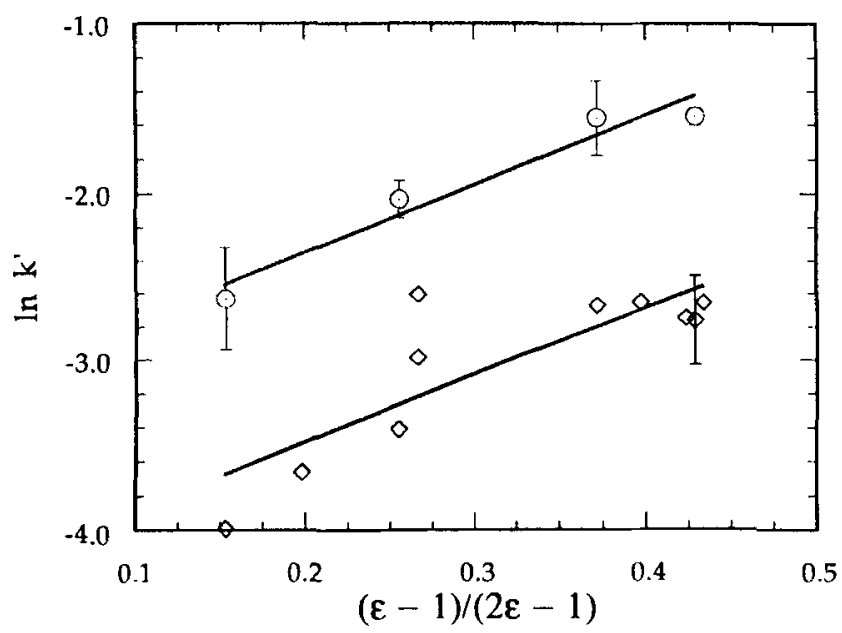

Figure 32. Kirkwood plot for oxidation kinetics in SC water at $380^{\circ} \mathrm{C}$.

Circles display data for phenol oxidation, and diamonds display data for 2-chlorophenol oxidation (Savage et al., $1994 \mathrm{a}$ ).

Courtesy of Technomic Publishing Co. pounds (Webley et al., 1991; Helling and Tester, 1988). Wightman (1981) also assumed reaction orders but took both the organic and the oxygen orders to be unity. Rate laws with assumed orders must be used with caution because they may omit or not properly account for the effects of reactant concentrations on the reaction rate.

Global rate laws have also been reported for the conversion of organic carbon to $\mathrm{CO}_{2}$ during the oxidation of phenol and 2-chlorophenol ( $\mathrm{Li}$ et al., 1992). Compared to the kinetics of substrate disappearance, the kinetics of $\mathrm{CO}_{2}$ formation were less sensitive to the organic concentration, more sensitive to the oxygen concentration, and insensitive to the water concentration.

3. Oxidation mechanisms. Global rate laws possess engineering utility, but they do not give much insight into the underlying chemistry. To understand SCWO reactions on a more fundamental level, one must deal with the oxidation mechanisms, which comprise a set of elementary reaction steps. At temperatures well above the critical point and approaching combustion temperatures, the oxidation reactions occurring during SCWO might be the same as those occurring during combustion. If so, one could develop fundamental SCWO reaction models by employing existing combustion models and accounting for the effect of higher pressures on the kinetics and accounting for the effect of a potentially nonideal fluid phase present in an SCWO system on the thermodynamic properties.

Groups at MIT, Los Alamos National Laboratory, and the University of Michigan have adopted this approach to develop detailed kinetics models. Tester's group at MIT reported the first such model, which was for $\mathrm{CO}$ oxidation (Helling and Tester, 1987). They, and others, have subsequently reported models for the oxidation of other simple compounds such as methane (Webley and Tester, 1991; Rofer and Streit, 1989; Rofer, 1991), methanol (Webley et al., 1990; Tester et al., 1992; Rofer and Streit, 1988), and hydrogen (Holgate and Tester, 1993, 1994b). The key postulate that motivated initial work with these simple compounds was that the free-radical oxidation mechanisms for more complex compounds would include the mechanisms for these simple compounds as key submodels. The oxidation of all hydrocarbons, for instance, could be expected to lead to $\mathrm{CO}$, which would then be oxidized to $\mathrm{CO}_{2}$. Thus, the $\mathrm{CO}$ oxidation mechanism becomes an important submodel. Likewise, the hydrogen resident in hydrocarbons would be oxidized to $\mathrm{H}_{2} \mathrm{O}$, so the $\mathrm{H}_{2}$ oxidation mechanism becomes another important submodel.

Much of the SCWO mechanistic modeling work done to date has had only limited successes in reproducing experimental results. An early model for $\mathrm{CO}$ oxidation failed to predict the formation of $\mathrm{H}_{2}$ (Helling and Tester, 1987). A model for methane (Webley and Tester, 1991) underpredicted methane conversions. An elementary reaction model for methanol (Webley et al., 1990) gave the correct conversions, but the activation energy was too high, and the $\mathrm{H}_{2}$, $\mathrm{CO}$, and $\mathrm{CO}_{2}$ product distributions were not correctly predicted. Other models for $\mathrm{CO}$ and methanol failed near the critical point of water (Rofer and Streit, 1989). The best success appears to have been achieved with a model of hydrogen oxidation (Holgate and Tester, 1993). The model predictions were generally consistent with experiments, and both the 
model and experiments gave the same global rate law parameters. The early mechanistic modeling papers previously cited suggested that the inability of accepted combustion models to describe SCWO implied that there must be some other, poorly understood effects, perhaps attributable to the presence of an SCF, responsible for the discrepancy between their models and experiments. These papers were cited by others (e.g., Cochran et al., 1992) as indicative of the need to understand the effects of local composition and solution microstructure, in this case by performing molecular dynamics calculations. The failure of well-established combustion mechanisms and rate constants is somewhat surprising, however, when one considers that many of the experiments performed were at sufficiently high temperatures that the fluid phase could be approximated as being an ideal gas. Moreover, at high temperatures (say $550^{\circ} \mathrm{C}$ ), far removed from the critical point, one would not expect exotic SCF phenomena to be important. This expectation is confirmed by experimental and modeling work on $n$-hexane pyrolysis reported by Dominé et al. (1990). They found that the elementary reaction steps and kinetics that were used to model hexane pyrolysis in the gas phase also gave good results when used to model the pyrolysis of SC hexane at conditions well above the critical point. They concluded that, for their system at least, "kinetic parameters determined in the gas phase remain valid in highly compressed supercritical fluids." Very recently, Tester's group (Holgate et al., 1994b) and our own group at Michigan (Brock and Savage, 1995; Savage et al., 1993) have reported detailed chemical kinetics models for the oxidation of simple compounds that are becoming more consistent with experimental results. These improved models took advantage of recent kinetics compilations for the elementary steps and properly handled the effect of pressure on the elementary reactions. Thus, it is apparent that elementary reaction models, when properly modified to account for pressure effects, may be able to give a good description of SCWO kinetics for simple compounds at conditions far removed from the critical point. This recent progress makes this approach appear promising. Of course, at conditions approaching the critical point, other features such as thermodynamics nonidealities (Bergan et al., $1989,1991)$ and SCF phenomena will need to be addressed in detailed chemical kinetics models. Progress in these areas and in in situ measurements of reacting molecules (Masten et al., 1993) and intermediates in SC water will facilitate development and validation of the next generation of these models.

After the mechanisms for these simple compounds are fully developed and verified, accurate models for the complete oxidation of more complex organics could be developed. The development of such detailed mechanistic models for actual organic pollutants would provide a fundamental reaction model that could be used with confidence to size equipment, to minimize the production of undesired intermediates, and to operate at the most efficient temperatures. Such predictive models could also reduce the need for expensive benchscale and pilot plant experiments. In this light, it is worthy of note that Gopalan and Savage (1993) have reported preliminary results from a detailed mechanistic model for the initial stages of the oxidation of phenol in SC water. Their model accounted for the important trends observed during the primary reactions of phenol oxidation. It was not a complete model, however, in that it did not account for ring-opening reactions, dimer destruction, and the ultimate transformation of organic carbon to $\mathrm{CO}_{2}$.

A mechanism-based, but philosophically different modeling approach has been described by Boock and Klein (1993). They present a lumping strategy that classifies elementary free-radical reactions according to type (such as hydrogen abstraction and isomerization) and then employs an EvansPolanyi correlation for each type. The adjustable parameters in the model are then determined by fitting experimental kinetics data. The authors demonstrated that this approach for modeling SCWO can fit experimental kinetics data for alcohols and acetic acid.

4. Heterogeneous reactions and SCWO. Several investigators have reported on the effects of different metals on SCWO reaction rates. Some of these studies were conducted to assess the role of heterogeneous reactions in nominally homogeneous systems, whereas others were deliberate attempts to accelerate the reaction rate via addition of a catalyst. An example of the former case is Thornton's (1991) test for the possibility of heterogeneous catalysis during phenol oxidation in SC water. He observed no effect of different metal surfaces (Hastelloy C-276, 316 stainless steel) or different surface-to-volume ratios on the conversion of phenol during SCWO in batch reactors. He concluded that the SCWO of phenol proceeded through homogeneous reactions. Webley et al. (1991), on the other hand, showed that SCWO of ammonia in a packed-bed reactor made of Inconel 625 was more rapid than oxidation in an unpacked tubular reactor made of Inconel 625 . They attributed this higher rate to catalysis by the Inconel 625 packing, which had increased the metal surface area by a factor of 30 . The reaction rate increased by only a factor of 4 , however, which suggested that there existed a dominant homogeneous reaction and a parallel heterogeneous reaction. In subsequent work, Holgate and Tester (1994a) found that added Inconel 625 inhibited the oxidation of $\mathrm{CO}$ and $\mathrm{H}_{2}$, presumably through increased termination reactions of free radicals. They concluded, however, that the contribution of this heterogeneous reaction to the kinetics observed in an unpacked tubular flow reactor was negligibly small. Although not dealing with oxidation reactions, the work of Yu et al. (1993) and Lira and McCrackin (1993) on reactions in SC water also explored the effects of different reactor surfaces. These investigations revealed different behavior in new and "aged" reactors and in Inconel and Hastelloy reactors. See the subsection titled Experiments with Single Compounds in the Conversion and Treatment of Biomass of this review for more details.

Yang and Eckert (1988) found that corrosion products from their Inconel 600 reactor served as homogeneous catalysts during the oxidation of $p$-chlorophenol. Metals contents as high as $30 \mathrm{ppm} \mathrm{Cu}$ and $3 \mathrm{ppm} \mathrm{Mn}$ were detected in the effluent stream. Deliberate addition of salts of these metals caused only a modest increase in reaction rate, but increasing the surface-to-volume ratio of the reactor caused a large increase in reaction rate. Chang et al. (1993) have also reported increases in the reaction rate with the addition of homogeneous metal saits.

Jin et al. $(1990,1992)$ studied the effect of a heterogeneous $\mathrm{V}_{2} \mathrm{O}_{5}$ catalyst on the supercritical water oxidation of 1,4-dichlorobenzene. They compared the conversions and $\mathrm{CO}_{2}$ yields obtained from catalytic SCWO with uncatalyzed SCWO 
and catalyzed gas-phase oxidation reactions. Their results indicated that SC water reduced the reaction rate. The authors suggested that water at high pressures influenced the adsorption equilibria and thereby inhibited the reaction rate.

Hydrothermal Flames. A new facet of combustion at supercritical conditions that has attracted attention only recently is combustion of a fuel with oxygen in a dense supercritical fluid. Aside from the considerable scientific interest in studying combustion and flames at high pressures and in unusual environments, there are potential applications of dense-phase combustion processes that include tertiary oil and gas recovery, toxic waste treatment by SCWO, and some aspects of jet propulsion. Using water as the fluid in densephase combustion experiments, as is typically done, can generate "hydrothermal" flames. Schilling and Franck (1988) were the first to report the existence of these hydrothermal flames. They used a high-pressure, corrosion-resistant flow cell fitted with sapphire windows for optical access. The nominal reaction conditions included pressures between 300 and $2,000 \mathrm{~atm}$ and temperatures of $400-500^{\circ} \mathrm{C}$. In some experiments, these investigators injected oxygen into a highpressure, supercritical mixture of methane and water and observed spontaneous ignition. The flame temperature, estimated from $\mathrm{OH}$ emission spectra, exceeded $3,000 \mathrm{~K}$. For a mixture with a methane-to-water mole fraction ratio of 0.3 to 0.7 , for example, spontaneous ignition occurred at $400^{\circ} \mathrm{C}$ when the pressure was $1,000 \mathrm{bar}$, at $405^{\circ} \mathrm{C}$ when $P=500 \mathrm{bar}$, and at $420^{\circ} \mathrm{C}$ when $P=200$ bar. No flames were observed at temperatures below $400^{\circ} \mathrm{C}$. Franck $(1991 \mathrm{a}, \mathrm{b})$ also noted that flames in dense supercritical media were observed when argon was used in place of water, and reported the generation of spontaneous hydrothermal flames from fuels other than methane and with air as the oxidant. He also showed that flameless combustion of methane leads to methanol, formic acid, and formaldehyde. We discuss these results and related work in the section titled Partial Oxidation.

Shortly after Schilling and Franck demonstrated hydrothermal combustion, Margolis and Johnston (1989) initiated a modeling study to examine the stability and multiplicity of hydrothermal flames in a nonadiabatic tubular reactor. Their model predicted that a steady planar flame could exist only within certain parameter ranges of the inflow velocity, the inlet and volumetric heat loss, the reactor length, and the global activation energy. Moreover, for a given flame position in the reactor, the model led to both high- and low-temperature solutions. The authors also explored the stability of these solutions.

Bechtold and Margolis (1992) modeled the structure of supercritical diffusion flames formed during the combustion of fuel and oxidizer in a high-density fluid such as SC water. They considered a counterflow configuration of fuel and oxidizer and the case of highly temperature-sensitive mass diffusivities (Arrhenius behavior). This model was an extension of Margolis and Williams' (1991) model for premixed supercritical flames. The results of Bechtold and Margolis showed that supercritical flames could be divided into three zones where the dominant phenomena were either pure convection, convection and diffusion, or diffusion and reaction. This categorization differed from those of flames with ordinary gases in that these have no pure convection zone. This zone arises in supercritical flames because of low Arrhenius mass diffusivi-

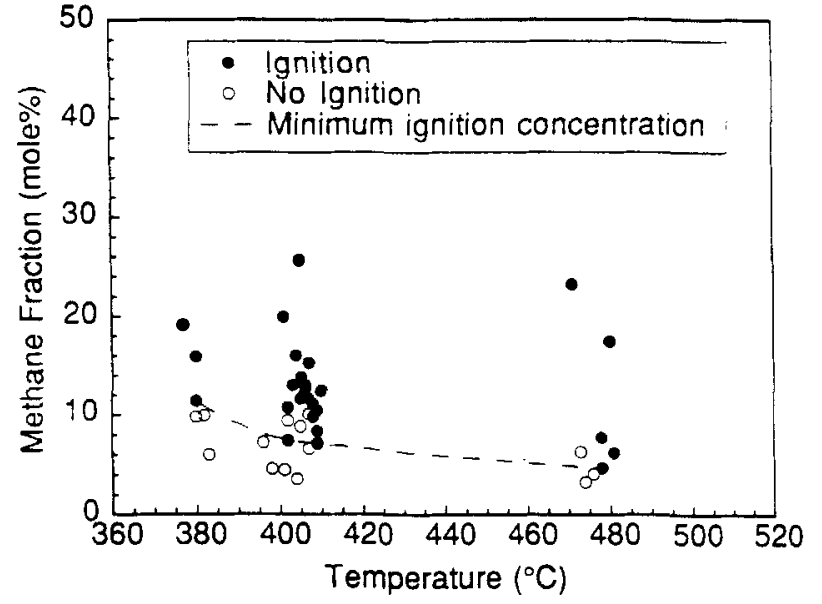

Figure 33. Explosive limits for methane in water (Steeper et al., 1992).

Courtesy of PRA Press.

ties at low temperatures. The stagnation temperature gave a characteristic S-shaped curve when plotted as a function of the Damköhler number. The ignition and extinction limits were the turning points on the curve.

Steeper et al. (1992) report a qualitative description of the structure of hydrothermal diffusion flames created when pure oxygen was injected into methane-water and methanol-water mixtures at a pressure of 275 bar and temperatures between $380^{\circ} \mathrm{C}$ and $510^{\circ} \mathrm{C}$. They used a hydrothermal flame reactor equipped with sapphire windows for a variety of optical diagnostics, including Raman spectroscopy. Steeper et al. (1992) also report the limits for spontaneous ignition of methane/oxygen and methanol/oxygen mixtures in SCW at 275 bar as functions of fuel mole fraction and temperature. Their data for methane are reproduced as Figure 33. The authors concluded that hydrothermal flames could possibly occur in commercial SCWO processes and upset system performance. Commercial SCWO reactors in which oxygen was injected directly into a supercritical stream of fuel/waste and water would most closely mimic the experimental apparatus. The potential occurrence of hydrothermal flames is an additional aspect to be taken into account when considering the safety requirements for a SCWO reactor.

Partial Oxidation. Partial oxidation reactions can be used to introduce oxygenated functional groups into hydrocarbons. These reactors are often used to synthesize chemicals, and there has been a limited amount of research done to assess the feasibility of using SCFs to replace more conventional gas- or liquid-phase oxidation processes. These studies are summarized below. Most of these studies also involve heterogeneous catalysts.

1. Partial oxidation of alkylaromatics and olefins. Dooley and Knopf (1987) postulated that SCF media might be advantageous for heterogeneously catalyzed partial oxidation reactions of alkylaromatics to phenols, aldehydes, and acids. They noted the possibility of having organics and oxygen in a single phase at reaction conditions, the viscosity and diffusivity of SCFs being intermediate to those properties in a gas or liquid, and the possibility of rapidly quenching the reaction by pressure letdown as motivation for considering SCF reac- 
tion media. The authors examined the partial oxidation of toluene in $\mathrm{SC} \mathrm{CO}_{2}$ as their test reaction. They first examined the phase behavior of the system and then conducted kinetics experiments. They reported that a supported $\mathrm{CoO}$ catalyst was both active and selective for the partial oxidation of toluene and generalized their results to conclude that alkylaromatic partial oxidation may be feasible in SCF reaction media. In subsequent work, Knopf et al. (1987) also examined the effect of pressure on the partial oxidation reaction. They found that the rate more than doubled as the pressure increased from 80 to $140 \mathrm{~atm}$. This pressure effect could not be attributed exclusively to the effect of pressure on the rate constant, which the authors estimated to cause only about a $30 \%$ increase in rate. Knopf et al. (1987) suggested that the balance of the pressure effect may be due to the variation of the $\mathrm{O}_{2}$ concentration in the catalyst pores with pressure. Pressure can influence adsorption and condensation in microporous materials, and the authors reasoned that the pores became enriched in $\mathrm{O}_{2}$ at higher pressures, thereby increasing the reaction rate.

Suppes et al. (1989) and Occhiogrosso and McHugh (1987) studied the partial oxidation of cumene-to-cumene hydroperoxide in $\mathrm{SC} \mathrm{CO}_{2}, \mathrm{Xe}$, and $\mathrm{Kr}$ at $110^{\circ} \mathrm{C}$. They examined the effect of different metals by using 316 stainless-steel and gold-plated reactors. This reaction is accomplished industrially via autoxidation of neat cumene in the liquid phase. The authors first identified the phase behavior of the system to ensure that their experiments were in the near-critical but single-phase region. They next conducted kinetics experiments and determined optimized values of the rate constants in the reaction mechanism. Their results showed that the conventional liquid-phase synthesis led to higher product yields than did partial oxidation in the SCFs. The authors suggested that eliminating the catalytic effect of metals on the termination steps during oxidation in SCFs may make the SCF-based process more favorable. These authors also noted that pressure had very little effect on the values of the rate constants, even near the mixture critical point. This insensitivity to pressure contrasts with the extreme sensitivity others (Johnston and Haynes, 1987) have reported for reactions in very dilute SCFs. Suppes et al. (1989) noted that the effect of pressure on the solution properties, and hence rate constants, should be much less dramatic for a mixture than for an infinitely dilute solution near its critical point.

Gaffney and Sofranko (1992) have reported on the feasibility of converting propylene to propylene glycol at supercritical conditions. Propylene glycol is an environmentally attractive replacement for ethylene glycol, and demand is expected to grow. Earlier work had shown that the desired product could be synthesized in aqueous solution, but corrosion was an expensive problem. The authors examined the feasibility of partial oxidation in a stream of propylene, water, and air in $\mathrm{CO}_{2}$ at supercritical conditions. Supercritical conditions were attractive because the rate can be influenced with pressure, the SCF can act as both a solvent and reagent, higher oxygen solubilities can be attained, and product separation can be facilitated. The authors evaluated several different catalysts and found that optimal results were obtained with a heterogeneous $\mathrm{CuI} / \mathrm{Cu}_{2} \mathrm{O} / \mathrm{MnO}_{2}$ catalyst supported on $\gamma$ $\mathrm{Al}_{2} \mathrm{O}_{3}$. The reaction conditions were $140^{\circ} \mathrm{C}$ and 2000 psig. Although the process was feasible, the production rate was

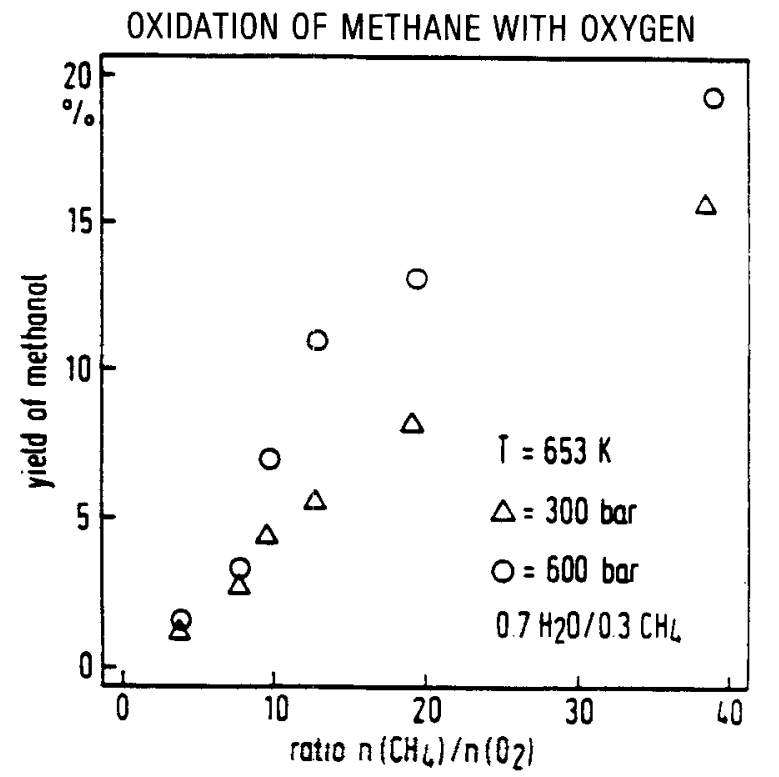

Figure 34. Fraction of methane reacted that forms methanol during partial oxidation in $\mathrm{SC}$ water at $653 \mathrm{~K}$ (Franck, 1991a).

about an order of magnitude too low to warrant further development.

2. Partial oxidation of methane. Franck (1991a) first showed that methane oxidation in SC water gives methanol as a detectable reaction product. He reported that up to $15-20 \%$ of the carbon in the methane converted appeared as methanol at $653 \mathrm{~K}$ and 300 and 600 bar. The selectivity increased with the methane-to-oxygen ratio and with pressure. Figure 34 gives these experimental results. Subsequent work (Hirth and Franck, 1993) showed that the methane disappearance kinetics were nearly pressure independent and that the addition of cobalt and platinum did not significantly increase the methanol selectivity.

Dixon and Abraham (1992) extrapolated the global kinetics for methane (Webley and Tester, 1991) and methanol (Tester et al., 1992) oxidation in SC water to temperatures near the critical point and noted that methane oxidation should be the more rapid. If this extrapolation is valid, then partial oxidation in SC water could overcome the fundamental problem of methane activation. Namely, that methane-derived products tend to be more reactive than methane. This observation led Dixon and Abraham to initiate experimental work on the conversion of methane to methanol in SC water. They used a $\mathrm{Cr}_{2} \mathrm{O}_{3}$ catalyst and reported the results of six experiments at $450^{\circ} \mathrm{C}$. The highest methanol yield they obtained was nearly $4 \%$, which corresponded to a methanol selectivity of about $40 \%$. The methanol yield increased as the initial ratio of methane to oxygen increased. The authors also reported a reaction network and pseudo-first-order rate constants that were consistent with their experimental data.

Savage et al. (1994b) also reported experimental results for the conversion of methane to methanol near the critical point of water. Unlike Dixon and Abraham, this group did not use a catalyst. The methanol selectivities ranged from 4 to $75 \%$, but the highest selectivities were obtained only at very low methane conversions of $<0.05 \%$. The highest methanol yield 
was $0.7 \%$. These authors also used Webley and Tester's (1991) mechanistic model for methane oxidation to explore the production of methanol from methane via partial oxidation in SC water. The model did not provide accurate predictions of the experimental data, but it did predict that the methanol yield would be very low over the parameter space investigated.

Supercritical Combustion. The behavior of fuel droplet vaporization and the interplay of chemical kinetics and transport phenomena in combustion systems at supercritical conditions have received attention because of long-standing interest in high-pressure combustion devices such as liquid propellant rocket motors and diesel engines. Williams (1973), Faeth (1977), and Hsieh et al. (1991) have provided reviews of the developments in supercritical combustion over the years. We will give only a very brief overview of the extensive work in this area here, and we refer the interested reader to these reviews for more information.

Experimental work often involved suspending a fuel droplet at the tip of a wire or thread, igniting the fuel, and monitoring variables such as the droplet temperature and size, the combustion lifetime, and the burning rate constant. Among the numerous experimental observations was that a change in the burning mechanism occurred at or near the critical pressure. The vaporization of the fuel droplet controlled the combustion process at subcritical pressures, whereas the combustion mechanism became diffusion controlled at supercritical pressures. Several groups have modeled combustion at supercritical conditions to identify and understand the important phenomena and to explain the behavior observed experimentally. Shuen et al. (1992) have published one of the most comprehensive models. They modeled the transient ignition and combustion of an isolated, initially subcritical, $n$ pentane fuel droplet when it is suddenly exposed to a stagnant oxidizing atmosphere at conditions that exceed the critical point of the fuel. The model featured conservation equations that incorporated variable transport and thermodynamic properties for the multicomponent system, a single global reaction rate law to account for the kinetics, and phase equilibrium considerations at the droplet interface. The model predicted that the droplet gasification rate would increase with pressure. It also predicted, consistent with experimental observations, that vaporization of the fuel dominates over burning at subcritical conditions, whereas combustion at supercritical conditions is a diffusion-controlled process. Similar models have been developed for combustion in a simpler slab geometry. Umemura (1986), for instance, considered the one-dimensional, isobaric, unsteady combustion of a liquid fuel and oxidizing gas initially in different spatially uniform states.

Fuel Stability. A trend in the development of high-performance aircraft is for the fuel to be the main heat sink. Thus, the temperatures a jet fuel experiences prior to combustion will rise and exceed the critical temperature of the fuel $\left(\sim 400^{\circ} \mathrm{C}\right)$. Fuel systems also typically operate at pressures ( $\sim 50 \mathrm{~atm})$ that exceed the critical pressure of most jet fuels. Fuels used under supercritical conditions must be stable. They must not cause fuel system problems such as surface deposits or filter plugging. The stability of existing jet fuels under supercritical conditions and the development of new, more stable fuels are areas of practical significance (Harrison and Hardy, 1992).

Edwards and Zabarnick (1993) tested the stability of different fuels and pure hydrocarbons under supercritical conditions. They found that the deposit-forming tendency of the fuel was a strong function of the temperature and flow rate through their test apparatus, but not a function of whether the fuel was above or below its critical temperature. They concluded that the deposition behavior was controlled by chemical reactions (autoxidation chemistry) and not by the changes in solvent properties that accompany the transition of a fluid from the subcritical to the supercritical regime.

\section{Electrochemistry}

In the early 1980s, several groups recognized the unique and adjustable properties of SCFs and examined them as media for electrochemical syntheses. Prior to this time, electrochemical investigations involving SCFs dealt exclusively with water and were focused primarily on corrosion studies. Silvestri et al. (1981) appear to be the first to report the use of SCF solvents for electrochemical processes. Their work investigated the use of $\mathrm{CO}_{2}, \mathrm{CF}_{3} \mathrm{Br}, \mathrm{HCl}$, and $\mathrm{NH}_{3}$ and the evaluation of the synthetic possibilities opened by these systems. They found that $\mathrm{SC} \mathrm{CO}_{2}$ and $\mathrm{CF}_{3} \mathrm{Br}$ were poor conductors and, hence, not good solvents for electrochemistry. $\mathrm{SC} \mathrm{HCl}$ was difficult to work with because of its corrosive nature, but Silvestri et al. did produce elemental iodine in the $\mathrm{SC} \mathrm{HCl/KI}$ system. SC ammonia appeared to be the best medium for electrochemistry, and these investigators accomplished the anodic dissolution of silver and iron in SC ammonia.

The most extensive work on electrochemistry in SCFs has been done by Bard and his colleagues at the University of Texas. Most of their experiments employed inorganic SCFs (water, ammonia), but they also demonstrated the feasibility of doing electrochemistry in an organic SCF by examining the reduction of phenazine and the oxidation of ferrocene in acetonitrile (Crooks and Bard, 1988b). The goal of their work was to understand the thermodynamics and kinetics of chemical reactions in SCFs and then use SCF solutions for electrosynthetic purposes. Additionally, by operating at supercritical conditions, these investigators were able to enter a new region of the parameter space and obtain previously unavailable thermodynamic data.

In initial work (Crooks et al., 1984) with $\mathrm{NH}_{3}$ they used cyclic voltammetry to generate and oxidize solvated electrons. They also used chronocoulometric experiments to determine the diffusivity of $m$-chloronitrobenzene in SC ammonia and noted that mass transport was enhanced at supercritical conditions. In subsequent articles (Crooks and Bard, 1987, 1988a) they reported on the reduction, dimerization, and diffusion of nitrogen-containing aromatics and nitrobenzene in ammonia. These reports were the first of electrochemistry of organics in SC ammonia. They found that reactions that occur in liquid ammonia can also be accomplished in SC ammonia. The advantage of operating at supercritical conditions, however, is that one can manipulate the dielectric constant, density, and viscosity of the solvent and hence engineer the reaction environment. The authors found that their results in SC ammonia were consistent with and could be explained in terms of earlier studies in liquid ammo- 
nia. They also measured diffusion coefficients in SC ammonia and found that the Stokes-Einstein equation applied. They concluded that the nature of diffusion and solvation between liquid and SC ammonia is not very different and that many of the concepts used to describe solvation in liquids can be applied to SCFs.

Crooks and Bard (1988a) studied the dimerization of quinoline and acridine radical anions in ammonia at temperatures between -70 and $150^{\circ} \mathrm{C}$. They selected this type of reaction because they expected it to be sensitive to changes in density and dielectric constant, which can be manipulated in SC ammonia by changing pressure or temperature. They used cyclic voltammetry to determine key kinetics and thermodynamics parameters for the reactions, and they discussed the experimental results in terms of the governing reaction mechanisms. They also measured diffusion coefficients for quinoline and acridine at different temperatures and found that the Stokes-Einstein equation gave a very good fit of the data. They noted that there were no dramatic differences in the reaction rates or products when the critical temperature was exceeded. Thus, there were no anomalies near the critical point.

Bard and coworkers have also examined the use of SC water as a medium for electrochemistry (Bard et al., 1988; Flarsheim et al., 1986, 1989; McDonald et al., 1986). The unique properties of water near its critical point render it a good solvent for both organics and salts. As such, it could compete with more expensive and hazardous organic solvents as a useful medium for electroorganic synthesis. The experimental difficulties associated with doing electrochemistry at conditions that exceed the critical point of water are legion, and they may have discouraged others from exploring this area. These workers discuss the experimental problems and describe the design, improvement, and operation of electrochemical cells for SC water. They verified that voltammetric techniques could be employed in near- and supercritical aqueous solutions to extract thermodynamic and kinetic data. The redox reactions they studied included the $\mathrm{I}^{-} / \mathrm{I}_{2}, \mathrm{Br}^{-} / \mathrm{Br}_{2}$, and hydroquinone/benzoquinone couples. This last couple demonstrates that it may be possible to do electrochemical syntheses of organic compounds in SC water. They also measured the diffusivity of copper ions, iodide ion, and hydroquinone at temperatures up to $375^{\circ} \mathrm{C}$ by chronoamperometry. The diffusivity increased with temperature and generally followed the expected Stokes-Einstein behavior. Interestingly, the measured diffusivities for hydroquinone and copper ions showed positive deviations from the Stokes-Einstein relation at the higher temperatures. This finding suggested that changes in solvation and the solvated ion hydrodynamic diameter might have occurred with increasing temperature. For the case of hydroquinone, a breakdown in hydrogen bonds between water and hydroquinone was noted as a possibility.

Flarsheim et al. (1989) took advantage of their experimental capabilities to examine the effect of pressure on the electrochemical half-reaction $\frac{1}{2} \mathrm{I}_{2}+e^{-} \leftrightarrow \mathbf{I}^{-}$in $\mathrm{SC}$ water at $385^{\circ} \mathrm{C}$. They reported that $\Delta \bar{v}_{r x n}$ varied from $-1030 \mathrm{~cm}^{3} / \mathrm{mol}$ at $230 \mathrm{bar}$ to $-290 \mathrm{~cm}^{3} / \mathrm{mol}$ at $300 \mathrm{bar}$. These large negative values indicated that the partial molar volume of the iodide ion was much more negative than the partial molar volume of $I_{2}$. This very negative partial molar volume resulted from the

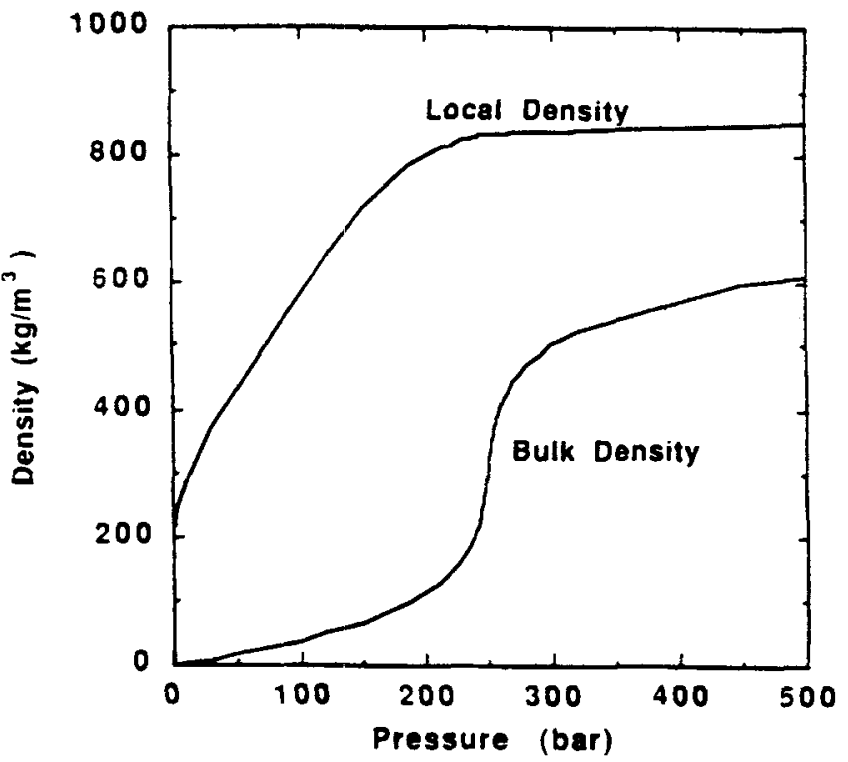

Figure 35. Local and bulk water density $2.2 \AA$ from the center of an ion at $385^{\circ} \mathrm{C}$ (Flarsheim et al., 1989).

Courtesy of American Chemical Society.

electrostatic attraction between iodide ions and water molecules. These attractive forces led to clustering of water molecules around the ion, and hence higher local densities and dielectric constants in the region near the ion. Figure 35 shows the calculated local density around the ion and compares it with the bulk density of water. It is clear that the local density is always higher, even at ambient pressure, and that the local density is much less pressure dependent in the near- and supercritical region than is the bulk density. The authors used the Carnahan-Starling-van der Waals equation of state and a modification of the Born equation to develop a quantitative theoretical model for the effects of pressure on $\Delta \bar{v}_{r x n}$. The resulting model accurately predicted the experimental measurements at pressures above 265 bar, but at lower pressures it predicted values much more negative than those actually measured. This discrepancy at lower pressures was attributed to the model's failure to account for ion pairing.

Wightman and coworkers oxidized ferrocene in SC carbon dioxide (Philips et al., 1987; Niehaus et al., 1989). They recognized that $\mathrm{CO}_{2}$ was a poor conductor and that conventional electrochemistry could not be accomplished in pure $\mathrm{CO}_{2}$. Nevertheless, the low critical temperature of carbon dioxide made it an appealing SCF to employ. Wightman's group found that adding water and tetrahexylammonium hexafluorophosphate to carbon dioxide produced a medium capable of supporting electrochemistry. They examined the effects of these additives on the oxidation of ferrocene and used voltammetry to assess the solvating power of the resulting near-critical fluid. They concluded that the addition of water affected the solvent properties of carbon dioxide and that the addition of the salt altered the diffusion rate of ferrocene. These results showed that additives can modify the properties of SC carbon dioxide, and this offers additional opportunities to manipulate the $\mathrm{SC}$ reaction environment. Later work (Niehaus et al., 1989) revealed that the addition of the salt led to the formation of a second fluid phase in the electro- 
chemical cell. The top phase was $\mathrm{CO}_{2}$ with ferrocene, and the bottom phase was the molten salt with ferrocene. They measured the partition coefficient of ferrocene between these phases and concluded that the presence of the salt improved voltammetry because the molten salt coated the electrode with a conducting layer.

Dombro et al. (1988) noted that the limited solubility of potential reactants and products in conventional electrolytes has hindered the development of commercial electrochemical syntheses. They advanced the use of SCFs as media for electrochemistry to circumvent this problem. More specifically, they demonstrate the feasibility of synthesizing dimethyl carbonate at $90^{\circ} \mathrm{C}$ from methanol and $\mathrm{CO}$ in an SC mixture of methanol and carbon dioxide. The current efficiency and selectivity to dimethyl carbonate were strong functions of the $\mathrm{CO}$ and electrolyte (tetrabutylammonium bromide) concentrations.

\section{Conversion and treatment of biomass}

The applications of SCFs in biomass utilization included in the present review are the use of SCFs as media for the delignification and pretreatment of wood, the liquefaction and degradation of biomass, and the dehydration of biomass fermentation products. In this section, we will first discuss investigations that used biomass or fractions thereof in experiments. Then we will review work done with model compounds, which was motivated by a desire to explain and better understand the results of experiments with biomass. $\mathrm{Li}$ and Kiran (1988) present a concise summary of investigations involving SCFs and lignocellulosic materials reported prior to 1987.

Wood Delignification and Pretreatment. Exploiting chemical reactions in SCFs to treat and convert biomass appears to have emerged from interest in SCF extraction of lignin from wood. Lignin removal is an important initial step in the pulping process, and strong chemicals and cooking processes are typically used in the pulp and paper industry. SCFs may offer the potential advantages of doing delignification at milder conditions and also recovering the lignin in a form amenable to further processing. Lignin is a cross-linked phenolic polymer, so it needs to be degraded into smaller fragments to become extractable. Beer and Peter (1985, 1986), Kiran (1987a,b), Li and Kiran (1988, 1989a,b), Reyes et al. (1989), Goto et al. (1990b), and Bludworth and Knopf (1993) have explored the use of near-critical and SC fluids for delignification.

Beer and Peter $(1985,1986)$ treated spruce wood with water/methylamine mixtures at temperatures between 150 and $180^{\circ} \mathrm{C}$. They examined the phase behavior of the system and reported that a mixture with $17 \%$ water was most effective for lignin extraction. The delignification kinetics appeared to consist of two distinct first-order regions with the delignification rate constant being high initially and then shifting to a lower value after the lignin content dropped below about $9 \%$. The authors noted that similar behavior had been observed for other delignification processes.

Kiran's group at the University of Maine studied the solubility, stability, and reactivity of wood species and models there of in a number of different SCFs (Kiran, 1987a,b; Li and Kiran, 1988, 1989a,b). Their goal was to understand the interactions of SCFs and lignocellulosic materials and then exploit this understanding to develop new processes. To develop a method for the reactive separation of lignin from wood using SCFs, Li and Karan (1989b) sought an SCF mixture with one component that could react with lignin and other components that would solubilize the lignin fragments so they could be extracted from the wood. They desired to operate the delignification at temperatures below $200^{\circ} \mathrm{C}$ to minimize the decomposition of the cellulose and hemicellulose components of wood. Therefore, the critical temperature of the mixture needed to be below $200^{\circ} \mathrm{C}$. Screening experiments (Li and Kiran, 1988) revealed that methyl amine/nitrous oxide mixtures were effective for delignification. These authors then delignified red spruce with methylamine and methyl amine/nitrous oxide mixtures under a wide range of different operating conditions ( $\mathrm{Li}$ and Kiran, 1989b). Their results showed that the extent of delignification increased with extraction time, temperature, pressure, and the methylamine mole fraction in the solvent mixture. An engineering model that accounted for the simultaneous reaction and diffusion occurring during the reactive separation of lignin from red spruce using this binary SC mixture was also reported ( $\mathrm{Li}$ and Kiran, 1989a).

A group at the University of California at Davis (Reyes et al., 1989; Goto et al., 1990b) explored the continuous reactive extraction of lignin from wood using SC tert-butylalcohol and isopropanol. Consistent with previous investigations, they observed that the extraction rate increased with both temperature and pressure (Figure 36) and that the delignification kinetics showed two first-order regimes. Goto et al. (1990b) modeled the extraction process using a reaction network that comprised two parallel first-order reactions. They reported the kinetics parameters and suggested that the two parallel reactions were needed in the model because lignin in different parts of the wood could react with different kinetics. These investigators also concluded that there was no influence of intraparticle diffusion on the extraction kinetics under their experimental conditions. They did note, however, that particle sizes for an industrial process would be much larger and that diffusion limitations were therefore more likely.

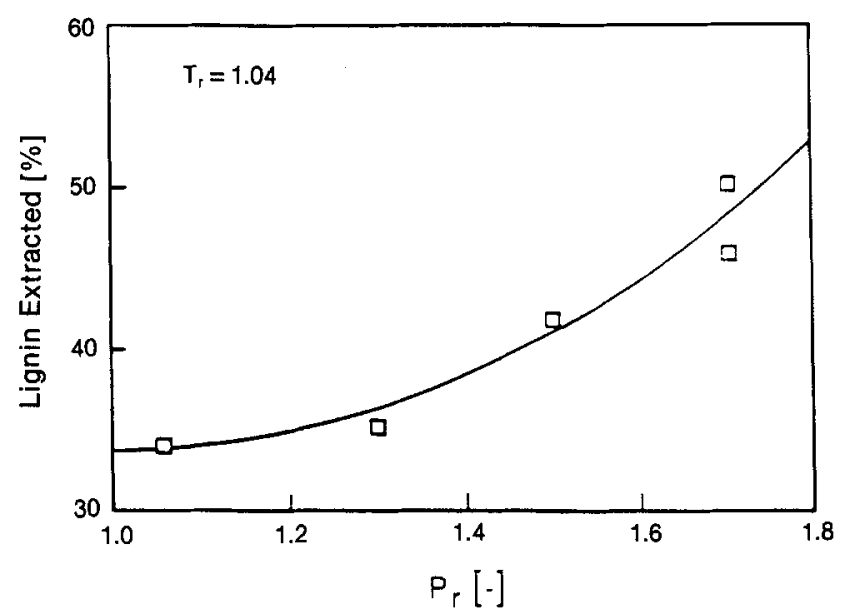

Figure 36. Effect of reduced pressure on lignin extraction by SC tertbutyl alcohol (Goto et al., 1990a).

Courtesy of American Chemical Society. 
Bludworth and Knopf (1993) used SC ammonia/water mixtures to extract lignin from yellow poplar. They used $\mathrm{NH}_{3}$ because it would be a less expensive reagent than methylamine, which $\mathrm{Li}$ and Kiran had found to be effective for delignification. Their experiments revealed that the water content and the temperature had the strongest influence on the amount of lignin extracted. For example, the authors reported $70 \%$ lignin removal during a one-hour extraction at $200^{\circ} \mathrm{C}$ and $272 \mathrm{~atm}$ with a $20 \%$ water solution. The extraction was not entirely selective, however, in that some of the hemicellulose and cellulose was also extracted. Bludworth and Knopf also developed a power-law rate expression for the delignification reaction, and they found the reaction to be 1.18 order in $\mathrm{NH}_{3}$ and -0.64 order in water, and to possess an activation energy of about $5.6 \mathrm{kcal} / \mathrm{mol}$.

Weimer et al. (1986) examined the reaction of wood with $\mathrm{SC}$ ammonia. This pretreatment technique makes some woods more amenable to a subsequent enzymatic hydrolysis process. They reported that ammonia at a density of $0.3 \mathrm{~g} / \mathrm{cm}^{3}$ and temperatures between 150 and $200^{\circ} \mathrm{C}$ reacted with the wood. Ammonolysis of ester linkages between lignin and carbohydrates was suggested as the key chemical reaction that made SC ammonia pretreatment effective for some woods.

Liquefaction and Degradation of Biomass. In addition to their use in traditional applications of biomass (pulp and paper), SCFs have also been investigated as vehicles for the liquefaction of biomass (conversion of these renewable resources to liquid fuels) and for the production of chemicals from biomass. Indeed, there has been steady interest in using renewable resources such as wood, cellulose, and lignin as a source of high-value chemicals or fuels. Lignin, for instance, is a phenolic polymer, so lignin "depolymerization" could be a route to phenols and related single-ring aromatics. Additionally, biomass is largely hydrocarbon, but with significant amounts of oxygen, so it could conceivably be converted to oils that could be upgraded to liquid hydrocarbon fuels. A key challenge to unlocking the chemicals and fuel value of biomass is to discover and develop effective conversion processes. Destructive distillation and pyrolysis have been used to convert the macromolecules in biomass to smaller molecules. Unfortunately, the nature of these thermal processes is such that the desired bond-breaking reactions that lead to potentially useful products occur along with undesired retrograde reactions that form char. Thus, alternate methods that employed liquefaction or solvent extraction (at times at supercritical conditions) have been explored in an attempt to increase oil yields and reduce char yields (Olcay et al., 1983; Beckman and Boocock, 1983; Boocock and Sherman, 1985; Modell, 1985; Calimi and Olcay, 1978, 1982; Köll et al., 1979; Labrecque et al., 1984; McDonald et al., 1983). Although some early work indicated that the SCF used in these processes was merely an inert diluent (Olcay et al., 1983), investigators recognized that since these nominal extractions occurred at elevated temperatures, solvolytic reactions between the biomass and SC solvent could also be occurring. The review of Subramaniam and McHugh (1986) discussed the area of biomass extraction, liquefaction, and degradation by highlighting the work of Köll et al. $(1979,1983)$ and Metzger et al. (1983). We will not discuss these articles here but rather will focus on the work published after and omitted from the previous review.
A group at Laval University (Labrecque et al., 1982, 1984; Porier et al., 1987) has explored the reactions of wood in SC methanol at temperatures up to $350^{\circ} \mathrm{C}$ and pressures up to 17 $\mathrm{MPa}$. Their goal was to convert wood to a liquid mixture (oil) that could be used as a fuel or as a source of chemicals. They used statistically designed experiments to examine the effects of several different process variables on the yield, composition, and molecular weight distribution of the oil, and on the yield of char and the wood conversion. The highest oil yields reported were about $63 \%$ at a wood conversion of $95 \%$. Their results showed that temperature, pressure, and the solvent flow rate were the most important factors. An increase in pressure, for instance, significantly increased the oil yield and the wood conversion, and decreased the char yield as shown in Figure 37. Labrecque et al. (1984) speculated that these effects could be attributed to the increasing solvent strength of the SCF with pressure or to the formation of solvent cages that trapped key reactive intermediates. The caged intermediates were more likely to follow unimolecular reactions that presumably formed the desired oils than to participate in the bimolecular reactions that were presumed to lead to char. A subsequent article by Poirier et al. (1987) attributed this pressure effect to the solvent power of the SCF. They suggested that more primary thermal degradation products were extracted from the wood before they could undergo charforming reactions as the pressure increased and methanol became a better solvent for these fragments. This group also reported that the SC methanol reacted with biomass fragments. They detected methylformate and methylacetate in the oils and suggested that these products arose from reactions between methanol and formic and acetic acids, respectively.

Modell (1985) reported results from the gasification and liquefaction of biomass in SC water. He showed that glucose was converted to liquid organics and gases rich in hydrogen and $\mathrm{CO}$ when processed near the critical point of water. Unlike processing at subcritical conditions, processing in $\mathrm{SC}$ water produced no char. Modell also found that the density of water had to be maintained near the critical density to prevent char formation. Experiments with cellulose and maple sawdust led to similar results and conclusions. Modell explained the effect of $\mathrm{SC}$ water by speculating that char formation was prevented because the SC water kept char precursors highly solvated and well dispersed. The rate of char-forming polymerization reactions were thereby inhibited. In addition to influencing the chemistry in its role as a solvent, SC water also participated as a reactant in chemical reactions with the biomass.

Cellulose accounts for about $40-50 \%$ of most biomass materials, so the use of SCFs to convert cellulose to chemicals or fuels has received attention. Goto et al. (1990a) used a flow system and SC $t$-butanol for the reactive extraction of volatiles and degradation products from cellulose at temperatures between 220 and $300^{\circ} \mathrm{C}$. Consistent with earlier investigators, they found that the presence of the SCF reduced char formation. The authors developed a global model wherein cellulose was first converted to "active" cellulose and then in parallel reactions to either soluble cellulose derivatives or to insoluble char. This global reaction network and pseudofirst-order kinetics gave a quantitative model that was able to capture the observed behavior.

Vick Roy and Converse (1985) used mixtures of $\mathrm{SO}_{2}$ and 

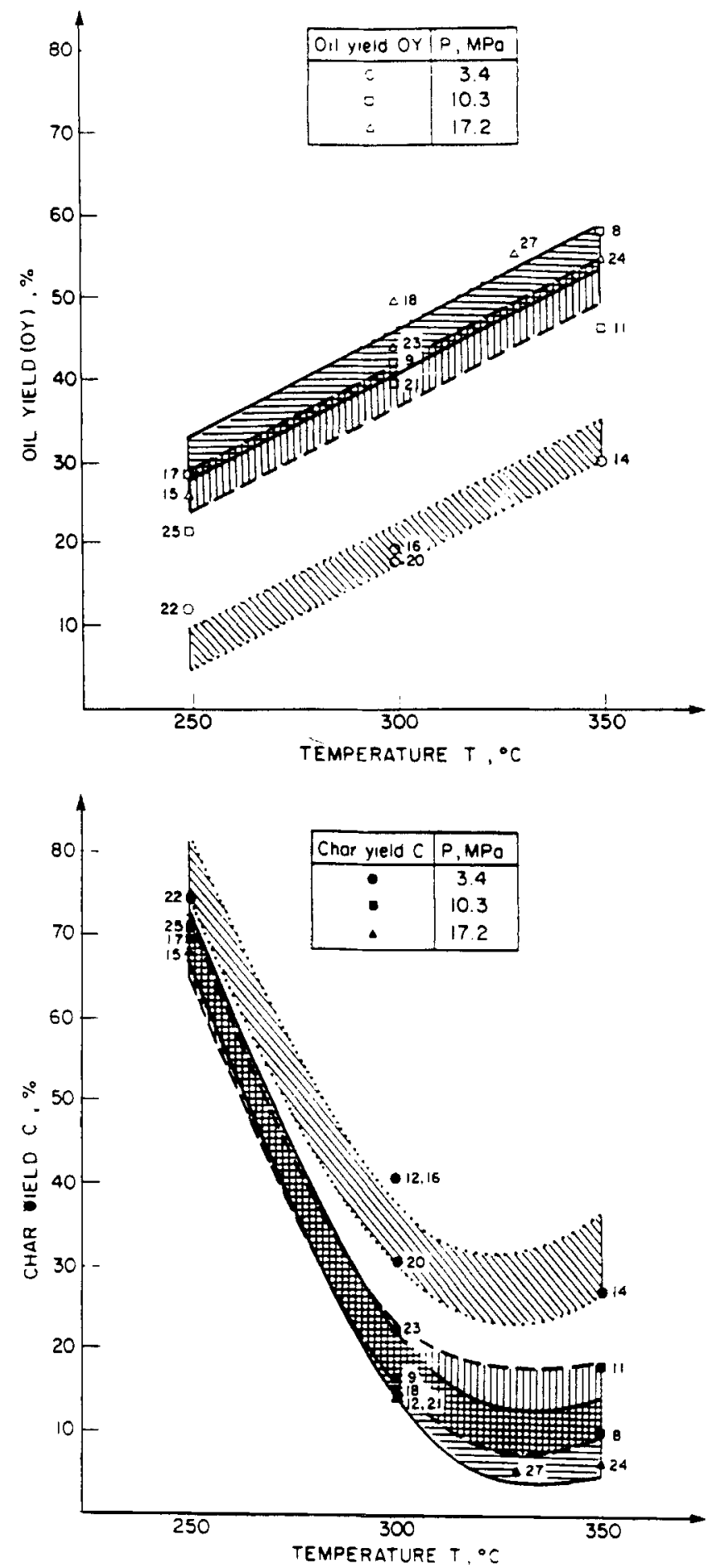

Figure 37. Effect of temperature and pressure on oil and char yield from wood liquefaction in methanol (Poirer et al., 1987).

Courtesy of American Chemical Society.

water above or near the critical point to convert cellulose (in wood) to glucose. The hydrolysis experiments were accomplished between 150 and $200^{\circ} \mathrm{C}$ and between 50 and $130 \mathrm{~atm}$ for batch holding times up to $30 \mathrm{~min}$. The maximum glucose yield $(42 \%)$ from delignified wood was obtained at subcritical conditions. Operating above the mixture critical point gave much lower yields ( $2 \%$ ), and the residual solids were charred.
It should be noted that these experiments at supercritical conditions typically employed higher temperatures and/or lesser amounts of water than did the subcritical experiments. These two factors probably account for the low glucose yields and char formation. The authors concluded that a hydrolysis process that used sulfur dioxide and water did not offer economic advantages over existing processes.

Mok and Antal (1990) explored the conversion of cellulose to glucose using water at near-critical temperatures and 5,000 psi (34 MPa) as the solvent. The advantages of using near-or supercritical conditions were given as reduced heat demands, reduced pretreatment demands, and the opportunity to do acid catalysis with low acid concentrations. They found that acid-catalyzed hydrolysis led to maximum glucose yieids of $55 \%$ at an optimal temperature of around $220^{\circ} \mathrm{C}$. They speculated that a solid-phase reaction competed with the conversion of cellulose to glucose and limited the maximum glucose yields to $55 \%$.

Lignin liquefaction using SC water was explored by $\mathrm{Fu}$ nazukuri et al. (1990). They found that the pyrolysis of a lignin sulfonate gave only a $2 \%$ oil yield, and that reaction under otherwise identical conditions but in the presence of SC water gave a nearly $30 \%$ oil yield. The oil yield reached this maximum of nearly $30 \%$ for a reaction at $400^{\circ} \mathrm{C}$ with $\rho_{w}=375$ $\mathrm{kg} / \mathrm{m}^{3}$ for about $3 \mathrm{~min}$. At longer times the oil yield decreased. The oil yields tended to increase with increasing water density, and this increase was most noticeable at densities less than $\rho_{c}$.

Experiments with Single Compounds. Most of the studies just discussed showed that SCFs could be potentially useful in the conversion of biomass to higher value products. These results motivated others to investigate the reaction fundamentals in an attempt to provide the reasons that SCFs were beneficial. Lawson and Klein (1985), for example, examined the influence of SC water on guaiacol (o-methoxyphenol) pyrolysis at $383^{\circ} \mathrm{C}$. Their motivation was to probe the contributions of pyrolysis during the extraction of volatiles from lignin with SC water. They used stainless-steel batch tubing-bomb reactors, tap water, and apparently loaded the reactors in air. Experiments with no water led to catechol and char as the major products, only traces of methanol, and phenol and $o$ cresol as minor products. Adding water increased the yields of catechol and methanol (see Figure 38), apparently at the expense of char. At high reduced water densities $\left(\rho_{r}=1.6\right)$, catechol and methanol were the major products. Lawson and Klein concluded that the water provided a parallel pathway (hydrolysis) for guaiacol that competed with the pyrolysis pathway. Since water is a reactant in the hydrolysis pathway, hydrolysis was favored at high water densities. The hydrolysis reaction was a continuous and increasing function of the water density, and there was no anomalous behavior near the critical point. The authors noted that the primary motivation for employing $\mathrm{SC}$ water in lignin extraction would be that the fluid is both hot and dense so hydrolysis would be rapid. The main applications of this work to lignin extraction in SC water were to identify water as a source of hydrogen and to note that processing in water affords the opportunity to obtain high yields of methanol, a desired product. The authors also noted that the guaiacol conversion went through a minimum as the water density increased, and they presented a reaction mechanism that accounted for this behavior. The 


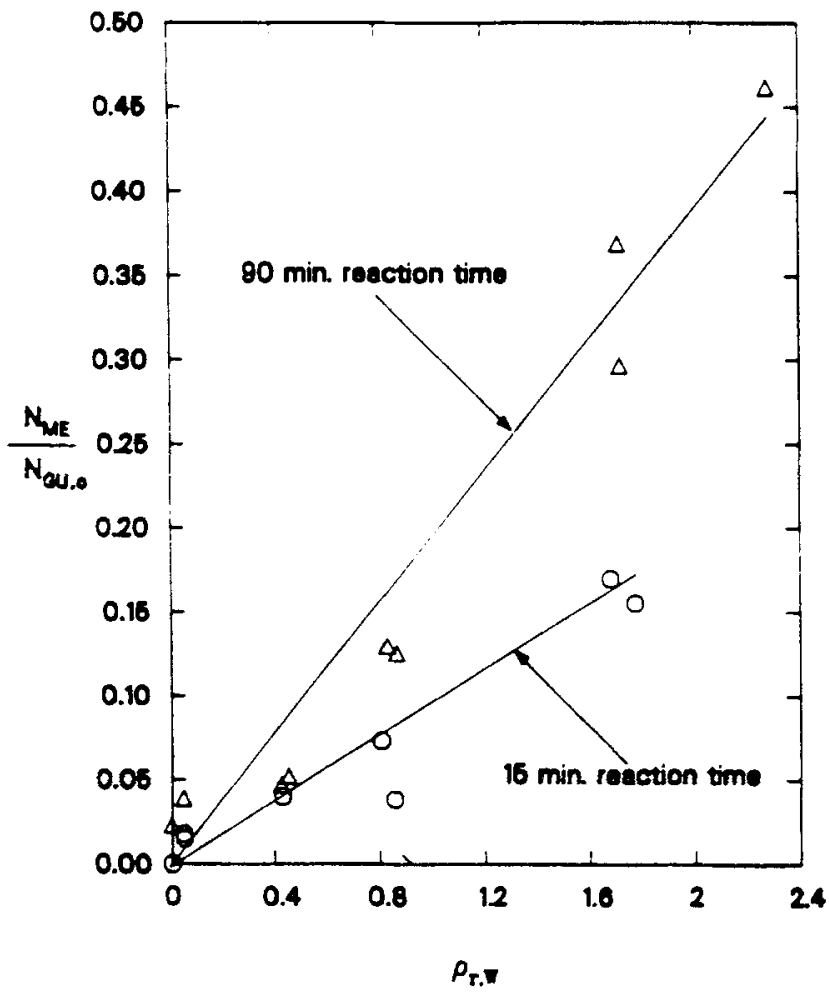

Figure 38. Effect of water density on methanol yield from guaiacol pyrolysis in water at $383^{\circ} \mathrm{C}$ (Lawson and Klein, 1985).

Courtesy of American Chemical Society.

mechanism invoked solvent cage effects, although the authors admitted these cage effects were speculative. A subsequent report (Huppert et al., 1989) on guaiacol hydrolysis in SC water was discussed in the section titled Coal Model Compounds.

In related work, Penninger and Kolmschate (1989) studied the reactions of 2-methoxynaphthalene, a model for aryl-alkyl ether bridges in biomass, in and with SC water. They used batch tubing-bomb reactors to explore the effects of density, ionic strength, and pressure on the pyrolysis and hydrolysis reactions at $390^{\circ} \mathrm{C}$. In the absence of water, the products present in highest selectivity were 2-naphthol, methylnaphthalene, methylnaphthol, $\mathrm{CO}$, methane, and polycondensates. At high water densities $\left(0.5 \mathrm{~g} / \mathrm{cm}^{3}\right)$, however, 2-naphthol and methanol, the hydrolysis products, were most abundant. The authors found that increasing the water density beyond its critical value increased the conversion and naphthol yields and decreased the yield of polycondensates. These observations are fully consistent with other reports (Lawson and Klein, 1985), wherein the presence of SC water shifted the dominant reaction pathway from pyrolysis to hydrolysis. Penninger and Kolmschate also observed that (1) the conversion increased with the addition of salts $(\mathrm{NaCl})$; (2) small amounts (68 ppm) of $\mathrm{HCl}$ increased the apparent global rate constant by an order of magnitude; (3) small amounts of a base $(\mathrm{NaOH})$ had no effect; and (4) the rate constant was linearly correlated with the square root of the ionic strength. These observations were consistent with the hydrolysis reaction proceeding through an acid-catalyzed, ionic mechanism. Finally, the authors also explored the effect of pressure on the rate constant and found that the activation volumes were between 2,377 and $3,300 \mathrm{~cm}^{3} / \mathrm{mol}$.

West and Gray (1987) pyrolyzed 1,3-butanediol, which mimics functional groups in lignocellulosic materials, in SC water at $425^{\circ} \mathrm{C}$ and reaction densities around $0.5 \mathrm{~g} / \mathrm{cm}^{3}$. They sought to explain previous observations that wood liquefaction in SC water led to higher yields of desired products and less char than did pyrolysis. These authors found that $\mathrm{SC}$ water had two main effects on the reaction. The first was to suppress the dehydration pathway that led to butenol and subsequent polymerization products, and the second was to alter the distribution of $\mathrm{C}_{1}-\mathrm{C}_{3}$ products. A 30-min reaction of the pure diol, for example, led to a "heavy liquids" yield of $15 \%$, and ethanol, acetone, methanol, methane, carbon dioxide, and propene as the most abundant $\mathrm{C}_{1}-\mathrm{C}_{3}$ products. Reacting an aqueous solution with $1 \mathrm{~mol} \%$ diol under the same conditions resulted in only a $6 \%$ heavy-liquids yield and formaldehyde and propene being the prominent $C_{3}-C_{3}$ products. $\mathrm{No}_{2}$ products were detected. West and Gray explained these results by speculating that the low dielectric constant of SC water (relative to ambient conditions) inhibited the presumably ionic dehydration reaction and that the water altered the relative rates of the butanediol fragmentation reactions to give mainly propylene and formaldehyde at the expense of the other $\mathrm{C}_{1}-\mathrm{C}_{3}$ products. The effects observed in the $1 \%$ aqueous solution cannot be attributed to the effects of concentration (competition between unimolecular and bimolecular processes) because the conversion of butanediol in the $1 \%$ solution varied from about 20 to $80 \%$ as the reaction density varied from 0.45 to about $0.65 \mathrm{~g} / \mathrm{mL}$. Figure 39 displays these data. Thus, it is clear that the water density (or pressure) influenced the kinetics and selectivity. These authors also explored the effect of the trace amount of oxygen dissolved in the liquid because it was exposed to room air on the conversion and product yields. They found that even these traces of oxygen significantly inhibited the reactions of 1,3-butanediol, and that this inhibition was most im-

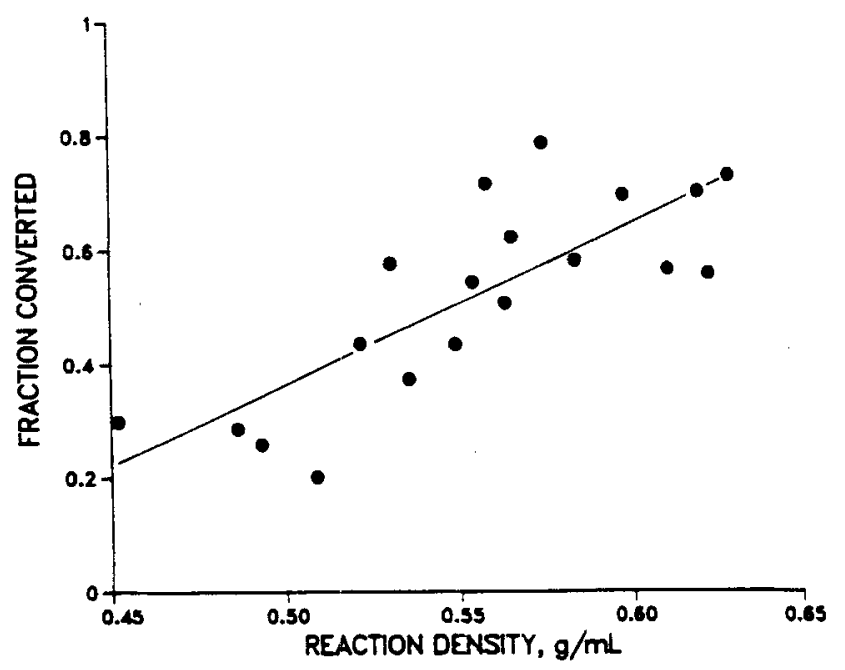

Figure 39. Effect of water density on conversion of 0.01 mole fraction 1,3-butanediol solution at $425^{\circ} \mathrm{C}$ for $30 \mathrm{~min}$ (West and Gray, 1987).

Courtesy of The Canadian Society for Chemical Engineering. 
portant when the diol concentration was low. This result led the authors to caution others in the field that "any attempt to investigate the quantitative kinetics of reactions in $\mathrm{SC}$ water must consider the effects of oxygen as a possible inhibitor."

A final body of literature with implications to biomass that deals with the reactions of individual compounds in a SCF is that of Antal's group at the University of Hawaii, which has done extensive work on the acid-catalyzed dehydration of alcohols, polyols, and carboxylic acids in SC water. These compounds serve as models of the $-\mathrm{OH}$ functionalities in cellulose polymers. The reactions of alcohols in water are also relevant to biomass conversion because fermentation processes can convert biomass to compounds such as ethanol or lactic acid. These useful products are typically present in low concentrations in aqueous solutions, leading to expensive separation and recovery steps. Converting the $-\mathrm{OH}$ containing compounds in the fermentation beer to the corresponding alkenes through acid-catalyzed dehydration reactions in $\mathrm{SC}$ water could be an attractive processing option. The compelling features of SC water for these applications are (1) at high densities $\left(>0.3 \mathrm{~g} / \mathrm{cm}^{3}\right)$ it remains a good medium for acid catalysis; (2) the product of the reaction (such as ethylene from ethanol) can be easily separated and recovered from the reactor effluent; and (3) the alkenes will be produced and hence available for use downstream (to make polyethylene) at high pressure. Additionally, operating near the critical point offers the opportunity to engineer the course of the reaction by manipulating temperature and pressure.

In their early work (Simkovic et al., 1987; Antal et al., 1987a,b; Ramayya et al., 1987; Mok et al., 1989), Antal's group demonstrated the feasibility of conducting dehydration reactions and performing acid catalysis in SC water by selectively converting ethanol to ethylene, $n$-propanol to propylene, ethylene glycol to acetaldehyde, glycerol to acrolein, and lactic acid to acrylic acid. They found that high yields of a single product could usually be obtained. Experiments were typically done in a flow reactor at temperatures around $375-400^{\circ} \mathrm{C}$ and at $34.5 \mathrm{MPa}$.

Having demonstrated the feasibility of dehydrating alcohols in SC water via acid catalysis, Antal and coworkers next sought to obtain the reaction kinetics data and mechanistic information required for process design, evaluation, optimization, and control. They reported that an E2 mechanism was consistent with their experimental kinetics results for both propanol and ethanol dehydration in SC water (Narayan and Antal, 1989; Xu et al., 1990), whereas an E1 mechanism was not consistent. The ethanol mechanism involved a protonated alcohol as an intermediate species, whereas the propanol mechanism may be concerted and avoid the formation of the charged intermediate (Narayan and Antal, 1990). They also reported rate constants for the elementary reaction steps for propanol at $375^{\circ} \mathrm{C}$ and $34.5 \mathrm{MPa}$ (Narayan and Antal, 1989) and for ethanol at $385^{\circ} \mathrm{C}$ and $34.5 \mathrm{MPa}$ (Xu et al., 1990, 1991). The propanol studies were extended to examine the influence of pressure on the kinetics at $375^{\circ} \mathrm{C}$ (Narayan and Antal, 1990). As the reduced pressure increased from 1.002 to 1.563 , the global rate constant decreased by a factor of 3. This influence of pressure on a rate constant can be interpreted in terms of an activation volume, but Narayan and Antal (1990) preferred to interpret the results in terms of the effect of pressure on the dielectric constant of SC water.

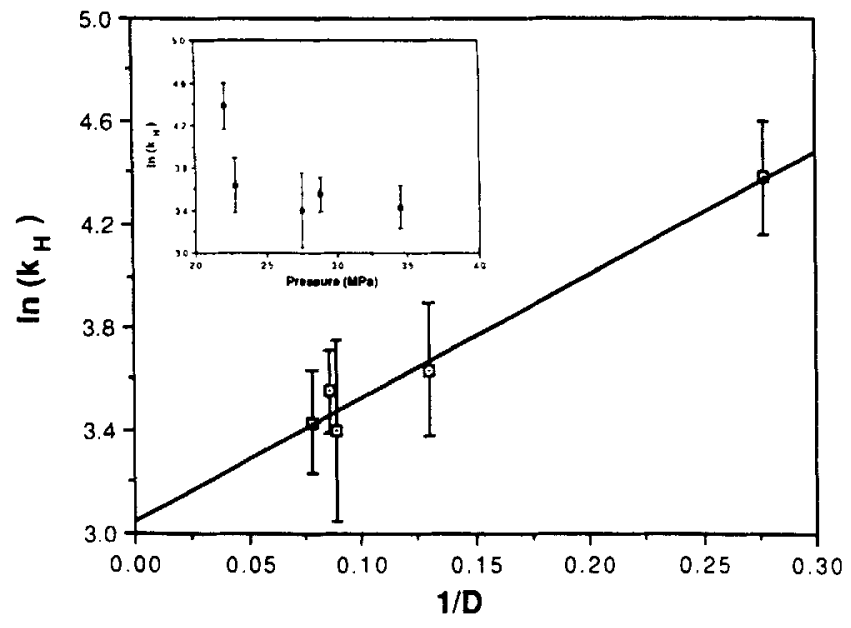

Figure 40. Effect of pressure and dielectric constant (D) on acid-catalyzed hydrolysis rate constant at $375^{\circ} \mathrm{C}$ (Narayan and Antal, 1990).

Courtesy of American Chemical Society.

Figure 40 displays their results. The dielectric constant increases with pressure, so the decrease in the rate constant with increasing pressure implied that the transition state species was less polar than the reactants. This finding was fully consistent with the dehydration reaction involving a concerted E2 mechanism. Such a mechanism was also consistent with experiments with added salts that showed that the ionic strength had no measurable effect on the dehydration kinetics. These articles provide a good SCF example of the importance of kinetics as a tool for mechanism discrimination when the data from well-designed experiments are carefully analyzed using statistical techniques.

$\mathrm{Yu}$ et al. (1993) reported on the gasification of glucose in SC water, where glucose served as a model carbohydrate in biomass. They found that the gasification efficiency for a 0.8 $\mathrm{M}$ glucose stream can exceed $85 \%$ at $600^{\circ} \mathrm{C}, 34.5 \mathrm{MPa}$, and $30-\mathrm{s}$ residence time in a Hastelloy $\mathrm{C} 276$ reactor. They also reported that the gasification reactions were strongly influenced by the reactor walls. Inconel strongly catalyzed the water-gas-shift reaction, whereas Hastelloy did not. A "corroded Hastelloy" reactor catalyzed the decomposition of acetic acid in SC water, whereas Inconel and new Hastelloy reactors did not.

A key general contribution from Antal and his students is that SC water can support both ionic and free-radical chemistry and that the operating conditions $(T$ and $P$ ) can be selected to favor one mechanism or the other. Antal et al. $(1987 \mathrm{a}, \mathrm{b})$ showed, for example, that ethanol with $0.01-\mathrm{M}$ $\mathrm{H}_{2} \mathrm{SO}_{4}$ catalyst reacts selectively to ethylene at $400^{\circ} \mathrm{C}$ and 34.5 $\mathrm{MPa}$, but the selectivity to ethylene is cut in half if the reaction is done at $500^{\circ} \mathrm{C}$. Hydrogen, carbon oxides, methane, and ethane also formed at $500^{\circ} \mathrm{C}$ in both the catalyzed and uncatalyzed reactions. Antal et al. (1987a,b) interpreted these results in terms of the ion product of water: $K_{w}$ was $7 \times 10^{-14}$ at $400^{\circ} \mathrm{C}$, and it was $2.1 \times 10^{-20}$ at $500^{\circ} \mathrm{C}$. On the basis of these and additional data, they concluded that ionic reactions dominated when $K_{w}>10^{-14}$ and that free-radical reactions dominated when $K_{w} \ll 10^{-14}$. We note, however, that even at $500^{\circ} \mathrm{C}, \mathrm{SC}$ water can apparently still support ionic chemistry because the addition of $0.01 \mathrm{M} \mathrm{H}_{2} \mathrm{SO}_{4}$ increased the 
ethanol conversion from $2 \%$ at a residence time $(\tau)$ of $66 \mathrm{~s}$ to $17 \%$ at $\tau=9 \mathrm{~s}$, and increased the selectivity to ethylene from 25 to $40 \%$. Thus, $K_{w} \ll 10^{-14}$ should not be used as a criterion to establish that ionic reactions cannot occur in SC water under a given set of reaction conditions.

Lira and McCrackin (1993) recently reported on the conversion of lactic acid to acrylic acid in near-critical water. Their study, which built on the earlier work of Mok et al. (1989), showed that $360^{\circ} \mathrm{C}$ was the optimal reaction temperature. They also noted that the addition of $\mathrm{Na}_{2} \mathrm{HPO}_{4}$ to the feed stream enhanced the rate of acrylic acid production, but more importantly, suppressed the rate constants for competing parallel reactions. The authors also noted a difference in the product distribution from new and "aged" Hastelloy C276 reactors.

\section{Materials}

SCFs have been used for different applications in materials processing. Most of these applications did not involve chemical reactions at supercritical conditions, however, and will not be reviewed here. For example, SCFs can be used to fractionate polydisperse polymers into narrow molecular weight ranges, to alter polymer morphology, to produce highly porous materials, and to produce particles with very narrow size distributions. McHugh and Krukonis $(1989,1994)$ discuss these physical processes involving materials and SCFs. We will confine the present review to solely chemical processes involving materials synthesis and decomposition reactions at supercritical conditions.

Polymerization and Depolymerization. Scholsky (1993) recently reviewed polymerization at supercritical conditions. We will not duplicate the material contained in this earlier review. Rather, we will give only a general discussion of the topic and then review only work that was not cited in Scholsky's review.

1. Polymerization. The attractive features of performing polymerization reactions at supercritical conditions are the same as those for other reaction systems at supercritical conditions. High pressure favors most addition polymerization reactions because these typically have a negative intrinsic activation volume. Additionally, the properties of SCFs (solubility parameter) are strong functions of density, and thus temperature and pressure, in the near-critical region, as Figure 41 illustrates. This "tunable" nature of SC solvents allows one to control the solubility of polymers of a given molecular weight in the reaction mixture and thereby control the molecular weight distribution with just small adjustments in the operating conditions. Further, the higher diffusivities of SCFs (relative to liquids) allow net reaction rates to be increased in situations where diffusion limitations are important. Finally, the lower viscosity of SCFs (relative to liquids) can reduce solvent cage effects that can hinder the efficiency of freeradical initiation reactions. The solvent may also interact with the transition state in such a way that it alters the kinetics of the polymerization reaction.

Most work reported on polymerization in SCFs falls into one of two classes. In some cases the reaction conditions exceed the critical point of the monomer (or comonomer), and in other cases the reaction conditions exceed the critical properties of an inert solvent. The latter case has seen more recent developments.

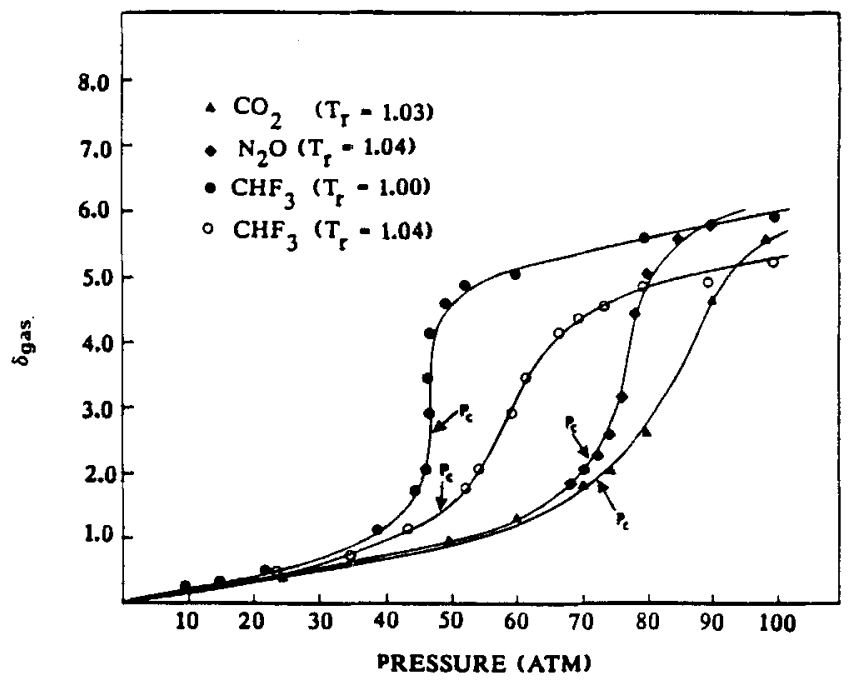

Figure 41. Solubility parameter as a function of pressure for different fluids near their critical temperature (Scholsky, 1993).

Courtesy of PRA Press.

The first and most successful polymerization at supercritical conditions was the high-pressure production of low-density polyethylene. Developed in the mid-1930s by ICI, the free-radical polymerization of ethylene remains largely unchanged since the $1950 \mathrm{~s}$. A free-radical initiator is added to the ethylene monomer at supercritical conditions [around 40,000 psia $(276 \mathrm{MPa})$ and $200-300^{\circ} \mathrm{C}$. The polyethylene chains remain in the SCF solution until the mixture is flashed to about $5000 \mathrm{psi}$, where the polymer precipitates. A key role of the SCF in this application, apart from being the reactant, is to keep the growing, high-molecular-weight polyethylene chains in solution. Ethylene can also be copolymerized with a wide variety of other monomers at supercritical conditions. Buback and Tups (1986), for example, report on the copolymerization of ethylene with $\mathrm{CO}$ to produce polyketones. Ahvenainen et al. (1992) describe a process for the copolymerization of ethylene in the presence of a Ziegler-Natta catalyst at supercritical conditions. There have also been a few developments in the polymerization of other olefins (propylene and tetrafluoroethylene) at SC conditions. Ethylene polymerization is a rare example of a commercial process based on SC polymerization. Most commercial-scale highpressure polymerizations occur in the liquid phase.

There has also been research into using inert SCFs in polymerization reactions. This concept has been tested only in the last ten years, so the synthesis of polymers in an inert SC medium has yet to be commercialized. Styrene has been polymerized in SC ethane, propane, and $n$-butane (Kumar et al., 1986; Kumar and Suter, 1987; Kiran and Saraf, 1990). This polymerization scheme accomplishes simultaneous reaction and fractionation because the polymers in the SCF phase become less soluble as they grow, and they eventually precipitate. Another advantage of polymerization at SC conditions is that recovery of unreacted monomer can be accomplished with small changes in the operating conditions. For liquidphase precipitation polymerization, monomer recovery is energy intensive. This work also demonstrated the feasibility of pressure-tuning in polymerization. Higher pressures pro- 
duced polymers with higher molecular weights. The effect of pressure was attributed to two factors. First, high pressures promote monomer addition to growing polymer chains and suppress termination. Second, high pressures give higher SCF densities so higher molecular-weight chains can remain in solution. Thus the effect of pressure on the molecular weight was attributed to both kinetic and thermodynamic phenomena.

With a different application in mind, Terry et al. (1988) polymerized light olefins in $\mathrm{SC} \mathrm{CO}_{2}$. They were investigating the feasibility of conducting polymerization reactions in $\mathrm{CO}_{2}$ in situ as the $\mathrm{CO}_{2}$ was used in enhanced oil recovery. Increasing the viscosity of the $\mathrm{CO}_{2}$ (via the generation of polymers) would allow the fluid to displace oil more effectively. Although the authors reported that ethylene and octene polymerized in $\mathrm{SC} \mathrm{CO}_{2}$, they did not observe the anticipated viscosity increase. A similar idea of using an SCF to transport a monomer within a porous material and then conduct the polymerization reaction at supercritical conditions is at the heart of a patent application by Sunol (1990). The patent describes the impregnation of wood and other porous materials with polymers to modify the material's mechanical properties.

Fluoropolymers have also been produced in $\mathrm{SC} \mathrm{CO}_{2}$. Fluoropolymers are insoluble in many common organic solvents, so they are typically produced in chlorofluorocarbon (CFC) solvents. Using an inert $\mathrm{SCF}$, such as $\mathrm{CO}_{2}$, as the reaction medium for the production of fluoropolymers can avoid the environmental problems associated with CFCs. DeSimone et al. (1992) demonstrated the homogeneous free-radical polymerization of 1,1-dihydroperfluoroctyl acrylate at $59.4^{\circ} \mathrm{C}$ and 207 bar, and its copolymerization with ethylene, styrene, butyl acetate, and methyl methacrylate in $\mathrm{SC} \mathrm{CO}_{2}$. In subsequent work, Guan et al. (1993) thoroughly studied the kinetics of the initiation step, decomposition of 2,2'azobis(isobutyronitrile), in $\mathrm{SC} \mathrm{CO}_{2}$. They found that the activation energy of $135 \mathrm{~kJ} / \mathrm{mol}$ for the apparent first-order rate constant was within the range of 120 to $140 \mathrm{~kJ} / \mathrm{mol}$ reported for liquid solvents. They also noted that the rate constant was lower in $\mathrm{SC} \mathrm{CO}_{2}$ than it was in benzene and attributed this difference to the lower dielectric constant in $\mathrm{SC} \mathrm{CO}_{2}$. The authors also presented a Kirkwood plot (Eq. 17 and Figure 42) of their kinetics data in $\mathrm{CO}_{2}$ and kinetics data in liquid solvents taken from the literature to support this interpretation. Additionally, the authors reported an intrinsic activation volume (which excluded solvation effects) of $23.6 \mathrm{~cm}^{3} / \mathrm{mol}$ based on experiments in liquid benzene. Finally, the authors developed a complete correlation of the effects of pressure on the observed decomposition rate constant. They accomplished this by noting that the dielectric constant was pressure-dependent and then combining the correlation from the Kirkwood plot with the effect of pressure on the rate constant expected from the intrinsic activation volume. This equation had the form of

$$
\ln k=-a+b\left(\frac{\epsilon(P)-1}{2 \epsilon(P)+1}\right)-\left(\frac{\Delta V_{\text {intr }}^{\ddagger}}{R T}\right) P \text {, }
$$

and Figure 43 shows that the model described the data in SC $\mathrm{CO}_{2}$. The first two terms in the equation come from the Kirkwood plot, and these account for pressure-dependent solvation effects.

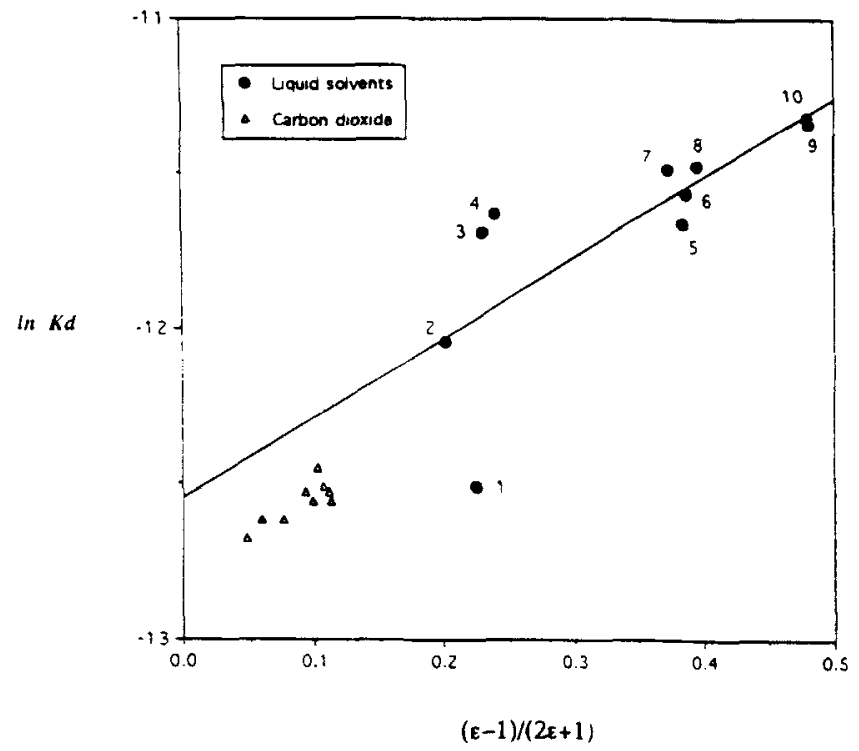

Figure 42. Kirkwood correlation for decomposition of azobis(isobutyronitrile) at $59.4^{\circ} \mathrm{C}$ in liquid solvents $(O)$ and $\mathrm{SC} \mathrm{CO}_{2}(\Delta)$ (Guan et al., 1993).

Courtesy of American Chemical Society.

Beckman and Smith (1990a,b) and Beckman et al. (1989) have studied inverse microemulsion polymerization reactions in near-critical and SC alkanes. They examined the phase behavior and microstructure of these inverse microemulsions and found that these features were a function primarily of the density of the continuous phase. This density dependence suggested that polymerization at supercritical conditions could offer opportunities to control the polymer size and

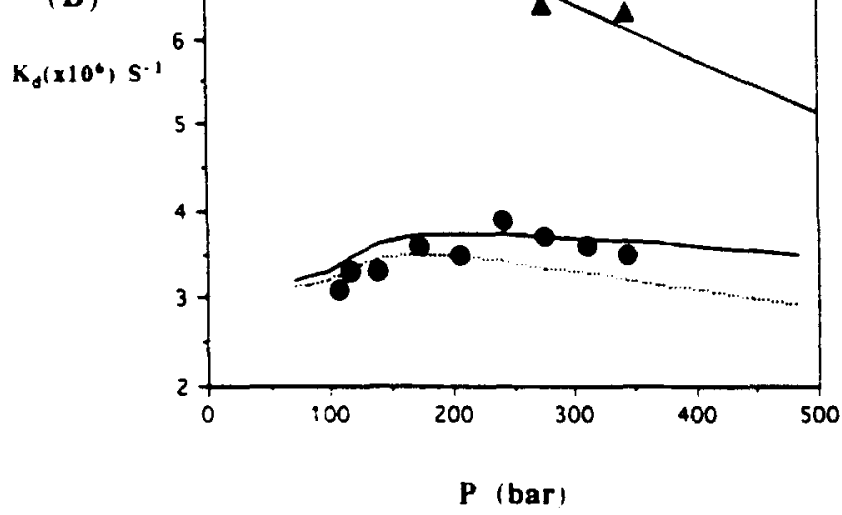

Figure 43. Rate constant for decomposition of azobis(isobutyronitrile) at $59.4^{\circ} \mathrm{C}$ and different pressures.

The filled triangles are data obtained from reactions in benzene, and the filled circles are from reactions in SC $\mathrm{CO}_{2}$. The curves through the $\mathrm{CO}_{2}$ data were determined from the model developed by Guan et al. (1993).

Courtesy of American Chemical Society. 
molecular weight by controlling density. The authors also reasoned that the higher diffusivity, lower viscosity, and higher pressure associated with a SCF could lead to higher reaction rates than those observed in liquid continuous phases. Furthermore, SCF polymerization could facilitate product recovery. These investigators polymerized acrylamide within a continuous phase of near-critical and SC mixtures of ethane and propane. Mixtures were used so that the density could be varied while holding temperature and pressure constant. The authors reported molecular weights ranging from 450,000 to nearly $1,000,000$ from polymerizations at $60^{\circ} \mathrm{C}$ and 362 bar. They also observed that increasing pressure had a large positive effect on the polymer yield. The authors indicated that the pressure effect was, in reality, a density effect because of the influence of density on the microstructure and phase behavior of the microemulsion. Increasing the density led to a decrease in the polymer molecular weight, but an increase in the rate of polymerization. The authors also noted that several of the features they observed for microemulsion polymerizations in SCFs were consistent with features observed for microemulsion polymerizations in liquid continuous phases.

Elliott and Cheung (1993) and Srinivasan and Elliott (1992) investigated the feasibility of producing polymeric microcellular foams (organic aerogels) via polymerization in near- and supercritical fluids. The authors polymerized methyl methacrylate with ethylene glycol-dimethacrylate in propane and Freon-22. They were attempting to combine the polymerization step and subsequent near-critical drying step into a single integrated process. The authors successfully demonstrated that these steps could be combined, but the resulting foams had undesirably high bulk densities.

One related application of SCFs as reaction media for polymerization is their use as part of a reaction suppression system. Lee and Hoy (1992) describe a method for suppressing reactivity by adding SCFs (primarily $\mathrm{CO}_{2}$ ) to a reacting mixture. The effect of the SCF seems to be that of diluting the mixture to reduce reaction rates.

2. Depolymerization and polymer modifications. Reports of polymerization reactions at supercritical conditions are numerous. More recently, research into using SCFs as reaction media for preparing polymer derivatives and for depolymerization has been reported. Yalpani (1993) prepared derivatives of polymers and biopolymers through chemical reactions in SCFs. The author sought an alternative to the conventional heterogeneous conditions that lead to nonuniformly derivatized materials. For example, chitosan reacted with glucose in $\mathrm{SC} \mathrm{CO}_{2}$ at $60^{\circ} \mathrm{C}$ to give a water-soluble chitosan derivative. $\mathrm{SC} \mathrm{CO}_{2}$ was also used as a medium for the phosphorylation of amylose and polyvinyl alcohol. The author speculated that the success of the polymer modification in $\mathrm{SC} \mathrm{CO}_{2}$ might be attributed to SCF-induced swelling or morphological changes in the polymer or to $\mathrm{pH}$ effects.

Another application of SCFs in polymer processing is conducting depolymerization reactions. Depolymerization of waste plastics is a potential means of handling these materials in an environmentally responsible way and also recovering the monomers or other potentially useful products. Pyrolytic depolymerization has some of the same drawbacks associated with the pyrolytic decomposition of coal (see the section titled Fuels Processing) or biomass (see the section titled Con- version and Treatment of Biomass): low yields of desired products, excessive char and gas formation, and little control over the reaction. The attractive features of SCFs for coal and biomass processing also motivated research into using SCFs in polymer decomposition. Dhawan et al. $(1991,1993)$ investigated the thermolysis of a styrene-butadiene copolymer in SC toluene and tetralin and the thermolysis of cispolyisoprene and scrap rubber in SC toluene. They also reported results from the reaction of SC toluene, tetralin, and their mixtures. Dhawan et al. found that the polymers could be degraded to low-molecular-weight products by thermolysis in SCFs. They also showed that secondary reactions that form polycondensates are remarkably suppressed relative to pyrolytic depolymerization of the same materials. Dhawan et al. (1991, 1993) identified over 100 different products in their depolymerization reactions, and many of these arose from reactions of the polymer with the SCF solvents used in their studies.

Materials Synthesis. The synthesis of nonpolymeric materials, such as fine metal oxide powders, in SCFs has recently been studied. These particles have many applications, including catalysts, coatings, ceramics, and electronic devices. Conventional methods for materials synthesis include sol-gel processes and hydrothermal processing. SCF processing was considered to be a potentially attractive alternative to sol-gel processes because of higher diffusivities and lower viscosities in the SCF phase, because the particle growth mechanism was expected to lead to fine and uniform size particles, and because SCF processing can simplify the process flowsheet. Hydrothermal processes have been used in the synthesis of quartz, emerald, and some magnetic metal oxides, but many of these processes employed subcritical reaction conditions (Laudise, 1987; Rabenau, 1985). Processing in SC water has received more attention recently, and it could be attractive because the density, viscosity, diffusivity, and dielectric constant of the reaction medium can be controlled through relatively small changes in temperature and pressure. This control and adjustability of the medium's properties might lead to control of the particle size, crystal structure, and morphology.

Pommier et al. (1990), Chhor et al. (1992) and Barj et al. (1992) have synthesized metal oxide ceramic powders $\left(\mathrm{MgAl}_{2} \mathrm{O}_{4}\right.$ and $\left.\mathrm{TiO}_{2}\right)$ from alkoxide precursors in $\mathrm{SC}$ ethanol. Ethanol was chosen because it is a good solvent for the metal alkoxide reactants and because its critical temperature is below the decomposition temperature of the compounds being investigated. Pommier et al. (1990) and Barj et al. (1992) decomposed the double alkoxide $\mathrm{Mg}\left[\mathrm{Al}(\mathrm{O}-\mathrm{sec}-\mathrm{Bu})_{4}\right]_{2}$ in $\mathrm{SC}$ ethanol to produce a submicron powder to be used as a precursor for the production of the spinel $\mathrm{MgAl}_{2} \mathrm{O}_{4}$. Characterization of the powders obtained from the conventional sol-gel process and from the SCF reaction revealed that the SCFproduced powder had a lower residual organic concentration and a $\mathrm{Mg} / \mathrm{Al} / \mathrm{O}$ ratio that much more closely resembled $\mathrm{MgAl}_{2} \mathrm{O}_{4}$. The presence of an SCF led to complex reaction mechanisms, believed to be a combination of hydrolysis and polycondensation, and possibly thermal decomposition. The authors also investigated the effects of reaction time and temperature. Chhor et al. (1992) synthesized $\mathrm{TiO}_{2}$ powders in SC ethanol and SC isopropanol. These powders were compared to those obtained from thermal decomposition in the 
vapor phase and from a sol-gel synthesis. The particles from the SCF process were submicron size and weakly associated into micron-sized agglomerates. The SC powders produced at $350^{\circ} \mathrm{C}$ were almost completely crystallized, so no additional treatment would be needed prior to sintering. This eliminates the need for additional thermal synthesis before sintering and avoids the extra settling-down and drying steps connected with the sol-gel process.

Adschiri et al. (1992a,b) explored the continuous synthesis of fine metal oxide particles using $\mathrm{SC}$ water as the reaction medium. The two overall reactions that lead from metal salts to metal oxides are (1) hydrolysis and (2) dehydration as illustrated below:

$$
\begin{aligned}
M\left(\mathrm{NO}_{3}\right)_{x}+x \mathrm{H}_{2} \mathrm{O} & =M(\mathrm{OH})_{x}+x \mathrm{HNO}_{3} \\
M(\mathrm{OH})_{x} & =M \mathrm{O}_{x / 2}+\frac{x}{2} \mathrm{H}_{2} \mathrm{O} .
\end{aligned}
$$

Processing in SC water allows these two steps to proceed at the same time, rather than sequentially as in conventional processing. Therefore, the dehydration step occurs while the particle size is small and the reaction rate is less affected by diffusion through the solid particle. The net effect is that the overall synthesis rate is very large. Other factors contributing to high reaction rates are the elevated temperature and the high diffusivity in SC water. Adschiri et al. synthesized several different metal oxide particles in $\mathrm{SC}$ water, including $\mathrm{AlOOH}, \mathrm{Fe}_{2} \mathrm{O}_{3}, \mathrm{Fe}_{3} \mathrm{O}_{4}, \mathrm{Co}_{3} \mathrm{O}_{4}, \mathrm{NiO}, \mathrm{ZrO}_{2}$, and $\mathrm{TiO}_{2}$. They also showed that the particle morphology could be controlled by varying the concentration and temperature. Moreover, the particle size could be adjusted by altering temperature or pressure. Fine particles with a narrow particle size distribution could be produced. The authors concluded that $\mathrm{SC}$ water may be an attractive reaction medium for the synthesis of metal oxide particles.

Yogo et al. (1989) and Hirano et al. (1991) synthesized metal-dispersed carbons by pyrolyzing metal-containing divinylbenzene copolymers in water at supercritical conditions. They report the synthesis of magnetite-dispersed carbon and nickel-zinc ferrite-dispersed carbon particles. These materials may have applications as catalysts and as magnetic materials. The organometallic polymers and copolymers were pyrolyzed in water at pressures between $30 \mathrm{MPa}$ and $125 \mathrm{MPa}$ and temperatures from 300 to $700^{\circ} \mathrm{C}$. Processing in SC water gave improved magnetic properties of the resultant carbon. The amount of water present also affected the morphology of the resultant material, presumably by influencing the phase separation of oligomers formed during the pyrolysis. The authors concluded that pyrolysis in SC water is a promising method for the production of oxide-dispersed carbon composites.

Nishikawa et al. (1991) and Nishikawa and Wakao (1992) studied the reactions of a platinum plate with germanium and germanium oxide in subcritical and SC water. The platinum/germanium compounds formed within the 20-40 $\mathrm{MPa}$ and 553-713 $\mathrm{K}$ range were $\mathrm{Pt}_{3} \mathrm{Ge}, \mathrm{Pt}_{3} \mathrm{Ge}_{2}, \mathrm{Pt}_{2} \mathrm{Ge}$, and $\mathrm{PtGe}$. Variations in the reaction temperature and pressure influenced which of these products crystallized on the platinum plate. The compositions of the crystals formed above the critical point of water were unique to those runs. The authors noted that the $\mathrm{Pt} / \mathrm{Ge}$ compounds and crystal sizes they observed were similar to those reported from the heat treatment of an etched Ge wafer upon which Pt had been sputtered.

Wood et al. (1992, 1993a,b) used SC ethylenediamene as the reaction medium to synthesize novel kinetically stabilized inorganic solid materials. These kinetically stabilized materials differ from the thermodynamically stable materials. The challenge in their synthesis is to provide intimate contact between the starting solid-state materials and enough thermal energy for the reaction to occur while avoiding high temperatures that favor formation of the thermodynamically stable products. Processing in SCFs offers an approach that may meet this challenge. The authors focused on the production of new Zintl phases. They reported a new solid-state, rodshaped compound $\mathrm{K}_{2} \mathrm{Ag}_{12} \mathrm{Se}_{7}$. The structure of this material contained continuous columns formed by infinite stacks of folded 18-member rings. This group also reported the synthesis of a novel phase of $\mathrm{M}_{2} \mathrm{Ag}_{6} \mathrm{~S}_{4}(M=\mathrm{Na}, \mathrm{K})$. They found that production of this phase occurred only when the ethylenediamine solvent was in the $\mathrm{SC}$ state during the reaction. Finally, Wood et al. (1993b) also prepared and studied $\left[\mathrm{W}_{4} \mathrm{~S}_{8}\left(\mathrm{H}_{2} \mathrm{NCH}_{2} \mathrm{CH}_{2} \mathrm{NH}_{2}\right)_{4}\right] \mathrm{S}$, which formed through the reaction of $\mathrm{K}_{2} \mathrm{~S}_{4}$ and $\mathrm{W}(\mathrm{CO})_{6}$ in $\mathrm{SC}$ ethylenediamine. This material was interesting because it contained lone sulfide atoms stabilized only by hydrogen bonding to chelated ethylenediamine molecules. These recent accounts published by Wood et al. (1992, 1993a,b) clearly show that using SCFs as reaction media for solid-state synthesis can lead to novel materials.

Energetic Materials. Supercritical fluids have been used as media for the decomposition of energetic materials. We referred to the work of Harradine et al. (1993) on the use of supercritical water oxidation to destroy energetic materials in the section titled Oxidation Reactions. This group viewed SCWO as a potential alternative to open burning or incineration for the safe destruction of materials such as propellants, explosives, and pyrotechnics. They examined the efficacy of SCWO and reported the destruction efficiencies, the byproducts formed, and the extent of reactor corrosion for a number of different compounds. Harradine et al. (1993) showed that SCWO can reduce pentaerythritol tetranitrate (PETN), 2,4,6-trinitrotoluene (TNT), cyclotetramethylene tetrani-tramine (HMX), cyclotrimethylene trinitramine (RDX), and nitroguanadine (NQ) levels to below detection limits via oxidation at admittedly extreme conditions of $600^{\circ} \mathrm{C}$ and $370 \mathrm{~atm}$, by using excess $\mathrm{H}_{2} \mathrm{O}_{2}$ as the oxidant. They found that corrosion of their Hastelloy C-276 reactor was not a problem for most of the compounds, but ammonium perchlorate was a notable exception. These authors also examined different methods for increasing the throughput of a largescale SCWO process. This issue is important because of the limited water solubility most energetic materials possess.

In a different set of studies relevant to the decomposition of energetic materials at SC conditions, Brower (1988) and Minier et al. (1991) have reported on the decomposition of nitromethane and nitroarenes. Brower (1988) examined the decomposition of nitromethane at SC conditions. He was interested in determining whether the pyrolysis chemistry might shift from a free radical to a polar mechanism as the density and dielectric constant of the reaction medium changed. The 
experimental results showed a dramatic increase in the firstorder rate constant as the nitromethane density increased. The rate constant at $400^{\circ} \mathrm{C}$ was $0.002 \mathrm{~s}^{-1}$ at $\rho=0.05 \mathrm{~g} / \mathrm{cm}^{3}$, but it increased 70 -fold to 0.141 at $\rho=0.56 \mathrm{~g} / \mathrm{cm}^{3}$. Brower suggested that there was a shift in the mechanism as the density increased and that the increase of rate with density can be linked to the increase in the dielectric constant. Other experimental evidence supporting the existence of a polar transition state for the first-order decomposition reaction was the low activation energy for pyrolysis at high densities $(0.6$ $\left.\mathrm{g} / \mathrm{cm}^{3}\right)$, the large, negative activation volume $(-85 \mathrm{ml} / \mathrm{mol})$, and the absence of a primary H/D kinetic isotope effect.

In a subsequent study, Minier et al. (1991) examined the thermal decomposition of nitroarenes in SC benzene and SC toluene. They used an SCF reaction medium so that they could take advantage of pressure-tuning and so that bimolecular reactions of the nitroarene with itself or its degradation products could be minimized. This latter consideration facilitated the development of probable elementary reaction steps and reaction mechanisms. The authors reported rate constants and mechanisms for the decomposition of several nitroarenes. The rate-determining step for nitrobenzene and $p$-nitrotoluene decomposition is hydrogen abstraction from the SC solvent. The authors showed that the measured activation volume, the $\mathrm{H} / \mathrm{D}$ kinetic isotope effect, and a linear free-energy relationship with the ionization potential of the solvent pointed to a transition state that involved a chargetransfer-type complex. This work provided additional insight into the decomposition of more complex nitroarenes, such as TNT, that are employed as energetic materials.

\section{Summary}

Fundamental and applied research into conducting chemical reactions at supercritical conditions has increased tremendously in the last eight years, as evidenced by the volume of literature cited in this review compared with that cited in Subramaniam and McHugh's review in 1986. Moreover, the diversity in this field is also evident in this review, where the wide range of potential applications include biochemical reactions, materials processing, pulp and paper production, fuels processing, catalysis, pollution prevention, and environmental control. Potential applications of reactions at SC conditions arise because of the unique properties of SCFs. The diffusivity of a molecule in an SCF falls between that in a liquid and in a gas. Thus, reactions that are diffusion limited in the liquid phase could become faster in an SCF phase. SCFs also have unique solubility properties. Compounds that are largely insoluble in a fluid at ambient conditions can become soluble in the fluid at supercritical conditions. This enhanced solubility can lead to higher reactant concentrations and hence accelerated reaction rates at SC conditions. This effect can also be exploited to form a single homogeneous fluid phase for a reaction and thereby overcome interphase transport limitations that might exist at subcritical conditions. Of course, phase behavior is important in SCF systems. Researchers and process developers need to verify that reactions are occurring in a single SCF phase, or, if multiple phases exist, to account explicitly for the reactions occurring in each phase. One additional feature of SCFs that makes them attractive as media for chemical reactions is that their properties vary with density, which is a strong function of temperature and pressure in the critical region. Consequently, SCFs provide the opportunity to engineer the reaction environment and to tune reaction rates, equilibrium, selectivities, and catalyst activity by manipulating temperature and pressure. This pressure dependence can also be used to integrate reaction and separation steps in a process flowsheet.

Many of the studies reviewed herein have employed many of the same applied kinetics tools that have been developed to understand and explain the behavior of chemical reactions in the gas phase and in solution. Kinetics models based on elementary reaction steps, cage effects, solvatochromism, salt effects, transition state theory, and the theory of diffusioncontrolled reactions all figure prominently in this review. Thus, it is apparent that the techniques and concepts routinely used to study reactions in solution can be profitably applied to reactions at supercritical conditions. Moreover, several investigations have reported that product yields and selectivities and reaction kinetics were well behaved as one passed from subcritical to supercritical conditions. These investigators observed no anomalies near the critical point, and the effects observed at near-critical and SC conditions could be explained on the basis of established solution-phase kinetics. We note here that the phenomena often associated with reactions at SC conditions (clustering, large activation volumes) appear to be most significant for dilute solutions very near the critical point. The effects become less significant as the solution becomes more concentrated (see Figure 8) and as one moves to temperatures and pressures well above $T_{c}$ and $P_{c}$ (Figures 8 and 10 ).

Finally, we conclude this review by noting that reactions at supercritical conditions will continue to be an active area of research and development. Some of this work will be driven by potential applications, as society continues to seek more efficient, more environmentally benign, or safer processes to make existing products and also seeks processes to make new products. Some work in this field will also continue to be driven by basic science, however, since SCFs are used to bridge the gap between gases and liquids and provide more detailed glimpses into the effects of solvents on chemical reactions. Studies of these fundamentals will give valuable information about molecular interactions and the behavior of medium-density fluids. In this light, investigations of fluid behavior and solvent effects on chemical reactions that use the same solvent over a very wide range of densities may make important fundamental contributions.

\section{Acknowledgments}

Our work on reactions at supercritical conditions has been supported by the National Science Foundation (CTS-9015738), the Department of Energy (DE-FG22-92PC92536), the Environmental Protection Agency Office of Exploratory Research (R817857-01-0), the Exxon Education Foundation, and The University of Michigan. We thank Dr. Ben Wu and Prof. Bala Subramaniam for helpful discussions about activation volumes and in situ coke extraction, respectively. We also thank Sandra Swisher and Cynthia Miller for assistance with preparing the manuscript.

\section{Notation \\ $\bar{V}=$ partial molar volume \\ $x=$ mole fraction}




\section{Subscripts and Superscripts}

$a=$ based on activity

$e=$ in excess of the bulk value

$r=$ reduced

$x=$ based on mole fractions

$\gamma=$ based on activity coefficients

$\infty=$ at infinite dilution

\section{Literature Cited}

Aaltonen, O., and M. Rantakylä, "Biocatalysis in Supercritical $\mathrm{CO}_{2}$," Chemtech, 21, 240 (Apr., 1991a).

Aaltonen, O., and M. Rantakylä, "Lipase Catalyzed Reactions of Chiral Esters in Supercritical Carbon Dioxide," Proc. Int. Symp. Supercritical Fluids, M. A. McHugh, ed., p. 146 (1991b).

Abraham, M. A., and M. T. Klein, "Pyrolysis of Benzylphenylamine Neat and With Tetralin, Methanol, and Water Solvents," Ind. Eng, Chem. Prod. Res. Dev., 24, 300 (1985).

Abraham, M. A., and M. T. Klein, "Solvent Effects during the Reaction of Coal Model Compounds," Supercritical Fluids: Chemical and Engineering Principles and Applications, T. G. Squires and M. E. Paulaitis, eds., ACS Symp. Ser., No. 329, p. 67 (1987).

Abraham, M. A., B. C. Wu, S. C. Paspek, and M. T. Klein, "Reactions of Dibenzylamine Neat and in Supercritical Fluid Solvents," Fuel Sci. Technol. Int., 6, 557 (1988).

Adschiri, T., S. Abe, and K. Arai, "Supercritical Fluid Extraction of Coal," Proc. Int. Symp. Supercritical Fluids, p. 408 (1991a).

Adschiri, T., S. Takaki, and K. Arai, "Catalytic Reforming of Coal Tar Pitch in Supercritical Fluid," Fuel, 70, 1483 (1991b).

Adschiri, T., A. Shinji, and K. Arai, "Catalytic Reforming of Coal Tar Pitch in Supercritical Fluid," Proc. Int. Symp. Supercritical Fluids, p. 408 (1991c).

Adschiri, T., K. Kanazawa, and K. Arai, "Rapid and Continuous Hydrothermal Crystallization of Metal Oxide Particles in Supercritical Water," J. Amer. Ceram. Soc., 75, 1019 (1992a).

Adschiri, T., K. Kanazawa, and K. Arai, "Rapid and Continuous Hydrothermal Synthesis of Boehmite Particles in Subcritical and Supercritical Water," J. Amer. Ceram. Soc., 75, 2615 (1992b).

Ahvenainen, A., K. Sarantila, and H. Andtsjo, "A Method for Homoor Copolymerizing Ethene," Int. Patent Appl. WO 92/12181 (1992).

Aida, T., and T. G. Squires, "Organic Chemistry in Supercritical Fluid Solvents: Photoisomerization of trans-Stibene," Supercritical Fluids, T. G. Squires and M. E. Paulaitis, eds., ACS Symp. Ser., 329 58 (1987).

Akao, K., and Y. Yoshimura, "Keto-enol Tautomeric Equilibrium of Acetylacetone in Trifluoromethane near the Critical Temperature," J. Chem. Phys., 94, 5243 (1991).

Amelse, J. A., and N. A. Kutz, "Catalyzed Xylene Isomeration Under Supercritical Temperature and Pressure Conditions," U.S. Patent 5,030,788 (1991).

Amestica, L., and E. E. Wolf, "Supercritical Liquefaction of an Illinois \#6 Coal," Proc. Int. Conf. Coal Sci., Center for Conf. Mgmt., Pittsburgh, p. 3 (1983).

Amestica, L. A., and E. E. Wolf, "Catalytic Liquefaction of Coal in Supercritical Tetralin," in Supercritical Fluid Technology, J. M. L. Penninger, M. Radosz, M. A. McHugh, and V. J. Krukonis, eds., Elsevier, Amsterdam, p. 331 (1985).

Amestica, L. A., and E. E. Wolf, "Catalytic Liquefaction of Coal with Supercritical Water/CO/Solvent Media,"Fuel, 65, 1226 (1986).

Antal, Jr., M. J., A. Brittain, C. DeAlmeida, S. Ramayya, and J. C. Roy, "Heterolysis and Homolysis in Supercritical Water," Supercritical Fluids, T. G. Squires and M. E. Paulaitis, eds., ACS Symp. Ser. No. 329 (1987a).

Antal, Jr., M. J., A. Brittain, C. DeAlmeida, W. Mok, and S. Ramayya, "Catalyzed and Uncatalyzed Conversion of Cellulose Biopolymer Model Compounds to Chemical Feedstocks in Supercritical Solvents," Energy Biomass Wastes, 10, 865 (1987b).

Asano, T., and W. J. LeNoble, "Activation and Reaction Volumes in Solution," Chem. Rev., 78, 407 (1978).

Balaban, M., S. Pekyardimci, M. Marshall, A. Arreola, J. S. Chen, and C. I. Wei, "Effects of High Pressure $\mathrm{CO}_{2}$ Treatment on Enzyme Activity in Model Systems and Orange Juice," Proc. Int. Symp. Supercritical Fluids, p. 114 (1991).
Balbuena, P. B., K. P. Johnston, and P. J. Rossky, "Molecular Simulation of a Chemical Reaction in Supercritical Water," J. Amer. Chem. Soc., 116, 2689 (1994).

Baldwin, R. M., and J. A. Manley, "Pyrolysis and Hydropyrolysis of Kentucky Oil Shale in Supercritical Toluene under Rapid Heating Conditions," Fuel Proc. Technol., 17, 201 (1988).

Baldwin, R. M., and K. W. Chen, "Pyrolysis and Hydropyrolysis of Two Carbonaceous Australian Oil Shales in Supercritical Toluene and Tetralin," Fuel, 66, 353 (1987).

Baldwin, R. M., G. S. Lane, and K. W. Chen, "Pyrolysis of Oil Shale in Supercritical Toluene: Reaction Mechanism and Role of Hydrogen," Fuel Proc. Technol., 13, 103 (1986).

Baptist-Nguyen, S., and B. Subramaniam, "Coking and Activity of Potous Catalysts in Supercritical Reaction Media," AIChE J., 38, 1027 (1992).

Bard, A. J., W. M. Flarsheim, and K. P. Johnston, "High Pressure Electrochemical Oxidation of Benzene at a Lead Dioxide Electrode in Aqueous Bisulfate Solutions at $25^{\circ} \mathrm{C}$ to $250^{\circ} \mathrm{C}, " J$. Electrochem. Soc., 135, 1939 (1988).

Barj, M., J. F. Bocquet, K. Chhor, and C. Pommier, "Submicronic $\mathrm{MgAl}_{2} \mathrm{O}_{4}$ Powder Synthesis in Supercritical Ethanol," J. Mat. Sci., 27, 2187 (1992).

Barner, H. E., C. Y. Huang, T. Johnson, G. Jacobs, M. A. Martch, and W. R. Killilea, "Supercritical Water Oxidation: An Emerging Technology," J. Haz. Mat., 31, 1 (1992).

Bartle, K. D., A. Calimli, D. W. Jones, R. S. Matthews, A. Olcay, H. Pakdel, and T. Tugrul, "Aromatic Products of $340^{\circ} \mathrm{C}$ Supercritical Toluene Extraction of Two Turkish Lignites: An NMR Study," Fuel, 58, 423 (1979).

Bartle, K. D., T. G. Martin, and D. F. Williams, "Chemical Nature of a Supercritical Gas Extract of Coal at $350^{\circ} \mathrm{C}$," Fuel, 54, 226 (1975).

Bechtold, J. K., and S. B. Margolis, "The Structure of Supercritical Diffusion Flames with Arrhenius Mass Diffusivities," Combust. Sci. Tech., 83, 257 (1992).

Beckman, D., and D. Boocock, "Liquefaction of Wood by Rapid Hydrolysis," Can. J. Chem. Eng., 61, 80 (1983).

Beckman, E. J., J. L. Fulton, D. W. Matson, and R. D. Smith, "Inverse Emulsion Polymerization of Acrylamide in Near-Critical and Supercritical Continuous Phases," Supercritical Fluid Science and Technology, ACS Symp. Ser. 406, p. 184 (1989).

Beckman, E. J., and R. D. Smith, "Microemulsion Polymerization Within Near- and Supercritical Continuous Phases: Effect of Microemulsion Structure on Reaction Characteristics," J. Supercrit. Fluids, 3, 205 (1990a).

Beckman, E. J., and R. D. Smith, "Phase Behavior of Inverse Microemulsions for the Polymerization of Acrylamide in Near-Critical and Supercritical Continuous Phases," J. Phys. Chem., 94, 345 (1990b).

Beer, R., and S. Peter, "High Pressure Extraction of Lignin from Wood," in Supercritical Fluid Technology, J. M. L. Penninger, M. Radosz, M. A. McHugh, and V. J. Krukonis, eds., Elsevier, Amsterdam, p. 385 (1985).

Beer, R., and S. Peter, "Lignin-Extraktion aus Holz mit Alkylaminen unter Druck," Chem. Ing. Tech., 58, 72 (1986).

Bergan, N. E., C. F. Melius, and H. A. Dwyer, "Studies of Thermodynamics Non-Ideality Effects on Chemical Equilibrium and Oxidation Reactions in Supercritical Fluids," Chem. Phys. Proc. Combust., 23, 1 (1989).

Bergan, N. E., Butler, P. B., and H. A. Dwyer, "High-Pressure Thermodynamics in Supercritical Water Oxidation Processes," Proc. Int. Symp. Supercritical Fluids, p. 169 (1991).

Bernard, P., D. Barth, and M. Perrut, "Internal Mass Transfer Limitation on Enzymatic Esterification in Supercritical Carbon Dioxide," High Pressure Biotech., 224, 451 (1992).

Bludworth, J., and F. C. Knopf, "Reactive Extraction of Lignin from Wood Using Supercritical Ammonia-Water Mixtures," J. Supercrit. Fluids, 6, 249 (1993).

Bolz, U., K. Stephan, P. Stylos, A. Riek, M. Rizzi, and M. Reuss, "Comparison of Enzymatic Catalysed Reactions in Organic Solvents and in Supercritical Fluids," Biochem. Eng.-Stuttgan Proc. Int. Symp., p. 82 (1991).

Boock, L., B. Wu, C. LaMarca, M. Klein, and S. Paspek, "Reactions in Supercritical Fluids," Chemtech., 23, 719 (Dec., 1992).

Boock, L. T., and M. T. Klein, "Lumping Strategy for Modeling the 
Oxidation of $\mathrm{C}_{1}-\mathrm{C}_{3}$ Alcohols and Acetic Acid in High-Temperature Water," Ind. Eng. Chem. Res, 32, 2464 (1993).

Boock, L., C. LaMarca, and M. T. Klein, "Hydrolysis and Oxidation in Supercritical Water," Endeavour, New Ser., 17, 180 (1993).

Boocock, D., and K. M. Sherman, "Further Aspects of Powdered Poplar Wood Liquefaction by Aqueous Hydrolysis," Can. J. Chem. Eng., 63, 627 (1985).

Bramlette, T. T., B. E. Mills, K. R. Hencken, M. E. Brynildson, S. C. Johnston, J. M. Hruby, H. C. Feemster, B. C. Odegard, and M. Modell, "Destruction of DOE/DP Surrogate Wastes with Supercritical Water Oxidation Technology," Sandia Nat. Lab. Rep SAND90-8229 (1990).

Brennecke, J. F., "Spectroscopic' Investigations of Reactions in Supercritical Fluids: A Review," in Supercrit. Fluid Eng. Sci., E. Kiran and J. F. Brennecke, eds., ACS Symp. Ser., p. 514 (1993).

Brennecke, J. F., and C. A. Eckert, "Phase Equilibria for Supercritical Fluid Process Design," AIChE J., 35, 1409 (1989a).

Brennecke, J. F., and C. A. Eckert, "Fluorescence Spectroscopy Studies of Intermolecular Interactions in Supercritical Fluids," in Supercritical Fluid Science and Technology, K. P. Johnston and J. M. L. Penninger, eds., ACS Symp. Ser., No. 406 (1989b).

Brennecke, J. F., P. G. Debenedetti, C. A. Eckert, and K. P. Johnston, AIChE J., 36, 1927 (1990a).

Brennecke, J. F., D. L. Tomasko, and C. A. Eckert, "Naphthalene/Triethylamine Exciplex and Pyrene Excimer Formation in Supercritical Fluid Solutions," J. Phys. Chem., 94, 7692 (1990b).

Brock, E. E., and P. E. Savage, "A Detailed Chemical Kinetics Model for the Supercritical Water Oxidation of $\mathrm{C}_{1}$ Compounds and $\mathrm{Hy}$ drogen," AIChE J., in press (1995).

Brower, K. R., "Thermolysis of Nitromethane in Pressurized Supercritical Media," J. Org. Chem., 53, 3776 (1988).

Bruno, T. J., "A Summary of the Patent Literature of Supercritical Fluid Technology (1982-1989)," in Supercritical Fluid Technology, T. J. Bruno and J. F. Ely, eds., CRC Press, Boca Raton, FL (1991).

Buback, M., and H. Tups, "High-Pressure Copolymerization of $\mathrm{C}_{2} \mathrm{H}_{4}$ and CO," Physica B \& C, $139 \& 140,626$ (1986).

Cahill, P., G. Harrison, and G. J. Lawson, "Extraction of Intermediate and Low-Rank Coals with Supercritical Toluene," Fuel, 68, 1152 (1989).

Calimli, A., and A. Olcay, "Supercritical Dioxane Extraction of Spruce Wood and of Dioxane-Lignin and Comparison of the Extracts with the Pyrolysis Products," Sep. Sci. Technol., 17, 183 (1982).

Carlier, C., and T. W. Randolph, "Dense-Gas Solvent-Solute Clusters at Near-Infinite Dilution: EPR Spectroscopic Evidence," AIChE J., 39, 876 (1993).

Castor, T. P., and G. T. Hong, "Critical Fluid Disruption of Microbial Cells," Proc. Int. Symp. Supercritical Fluids, p. 139 (1991).

Ceylan, R., and A. Olcay, "Supercritical Gas Extraction of Turkish Coking Coal," Fuel, 60, 197 (1981).

Chang, K.-C., L. Li, and E. F. Gloyna, "Supercritical Water Oxidation of Acetic Acid by Potassium Permanganate," J. Haz. Mat., 33, 51 (1993).

Chateauneuf, J. E., C. B. Roberts, and J. F. Brennecke, "Laser Flash Photolysis Studies of Benzophenone in Supercritical $\mathrm{CO}_{2}$," Supercritical Fluid Science and Technology: Theoretical and Applied Approaches in Analytical Chemistry, F. V. Bright and M. P. McNally, eds., ACS Symp. Ser., No. 488, 106 (1992).

Chhor, K., J. F. Bocquet, and C. Pommier, "Synthesis of Submicron $\mathrm{TiO}_{2}$ Powders in Vapor, Liquid and Supercritical Phases, a Comparative Study," Mat. Chem. Phys., 32, 249 (1992).

Chi, Y. M., K. Nakamura, and T. Yano, "Enzymatic Intersterification in Supercritical Carbon Dioxide," Agric. Biol. Chem., 52, 1541 (1988).

Chialvo, A. A., and P. G. Debenedetti, "Molecular Dynamics Study of Solute-Solute Microstructure in Attractive and Repulsive Supercritical Mixtures," Ind. Eng. Chem. Res., 31, 1391 (1992).

Cochran, H. D., P. T. Cummings, and S. Karaborni, "Solvation in Supercritical Water," Fluid Phase Equil., 71, 1 (1992).

Collins, N. A., P. G. Debenedetti, and S. Sundaresan, "Disproportionation of Toluene over ZSM-5 under Near-Critical Conditions," AIChE J., 34, 1211 (1988).

Combes, J. R., K. P. Johnston, K. E. O'Shea, and M. A. Fox, "Influence of Solvent-Solute and Solute-Solute Clustering on Chemical Reactions in Supercritical Fluids," Supercritical Fluid Science and Technology: Theoretical and Applied Approaches in Analytical Chemistry, F. V. Bright and M. P. McNally, eds., ACS Symp. Ser. 488, p. 31 (1992).

Connors, K. A., Chemical Kinetics: The Study of Reaction Rates in Solution, VCH Publishers, New York (1990).

Crain, N., S. Tebbal, X. Li, and E. F. Gloyna, "Kinetics and Reaction Pathways of Pyridine Oxidation in Supercritical Water," Ind. Eng. Chem. Res., 32, 2259 (1993).

Crooks, R. M., F. F. Fan, and A. J. Bard, "Electrochemistry in Near-Critical and Supercritical Fluids: 1. Ammonia," J. Amer. Chem. Soc., 106, 6851 (1984).

Crooks, R. M., and A. J. Bard, "Electrochemistry in Near-Critical and Supercritical Fluids: 4. Nitrogen Heterocycles, Nitrobenzene, and Solvated Electrons in Ammonia at Temperatures to $150^{\circ} \mathrm{C}, " J$. Phys. Chem., 91, 1274 (1987).

Crooks, R. M., and A. J. Bard, "Electrochemistry in Near-Critical and Supercritical Fluids: 5. The Dimerization of Quinoline and Acridine Radical Anions and Dianions in Ammonia from $-70^{\circ} \mathrm{C}$ to $150^{\circ} \mathrm{C}$," J. Electroanal. Chem., 240, 253 (1988a).

Crooks, R. M., and A. J. Bard, "Electrochemistry in Near-Critical and Supercritical Fluids: 6 . The Electrochemistry of Ferrocene and Phenazine in Acetonitrile between 25 and $300^{\circ} \mathrm{C}$," J. Electroanal. Chem., 243, 117 (1988b).

Debenedetti, P. G., "Clustering in Dilute, Binary Supercritical Mixtures: A Fluctuation Analysis," Chem. Eng. Sci., 42, 2203 (1987).

Dell'orco, P. C., B. R. Foy, J. M. Robinson, and S. J. Buelow, "Hydrothermal Treatment of Hanford Waste Constituents," Haz. Waste Haz. Mater., 10, 221 (1993).

Deshpande, G. V., G. D. Holder, A. A. Bishop, J. Gopal, and I. Wender, "Extraction of Coal Using Supercritical Water," Fuel, 63, 956 (1984).

Deshpande, G. V., G. V. Holder, and Y. T. Shah, "Kinetics of Coal Liquefaction under Supercritical Conditions," Ind. Eng. Chem. Proc. Des. Dev., 25, 705 (1986).

DeSimone, J. M., Z. Guan, and C. S. Elsbernd, "Synthesis of Fluoropolymers in Supercritical Carbon Dioxide," Science, 257, 945 (1992).

Dhawan, J. C., A. F. Bencsath, and R. C. Legendre, "Depolymerization Reactions of cis-Polyisoprene and Scrap Rubber in Supercritical Toluene," Supercritical Fluid Engineering Science, ACS Symp. Ser. 514, p. 380 (1993).

Dhawan, J. C., L. C. Legendre, A. F. Bencsath, and R. M. Davis, "Reaction Products of Styrene-Butadiene ABA Block Copolymer in Supercritical Toluene and Tetralin," J. Supercrit. Fluids, 4, 160 (1991).

Dixon, C. N., and M. A. Abraham, "Conversion of Methane to Methanol by Catalytic Supercritical Water Oxidation," J. Supercrit. Fluids, 5, 269 (1992).

Dombro, R. A., G. A. Prentice, and M. A. McHugh, "Electro-Organic Synthesis in Supercritical Organic Mixtures," J. Electrochem. Soc., 135, 2219 (1988).

Dominé, F., P. Marquaire, C. Muller, and G. Côme, "Kinetics of Hexane Pyrolysis at Very High Pressures 2. Computer Modeling," Energy Fuels, 4, 2 (1990).

Dooley, K. M., S. R. Brodt, and F. C. Knopf, "Comments on 'Reactions in Supercritical Fluids-A Review'," Ind. Eng. Chem. Res., 26, 1267 (1987).

Dooley, K. M., and F. C. Knopf, "Oxidation Catalysis in a Supercritical Fluid Medium," Ind. Eng. Chem. Res., 26, 1910 (1987).

Dumont, T. D., D. Barth, and M. Perrut, "Continuous Synthesis of Ethyl Myristate by Enzymatic Reactions in Supercritical Carbon Dioxide," Proc. Int. Symp. Supercritical Fluids, p. 150 (1991).

Dumont, T., D. Barth, and M. Perrut, "Continuous Synthesis of Ethyl Myristate by Enzymatic Reaction in Supercritical Carbon Dioxide," J. Supercrit. Fluids, 6, 85 (1993).

Dumont, T., D. Barth, C. Corbier, G. Branlant, and M. Perrut, "Enzymatic Reaction Kinetic: Comparison in an Organic Solvent and in Supercritical Carbon Dioxide," Biotech. Bioeng., 39, 329 (1992).

Eckert, C. A., C. K. Hsieh, and J. R. McCabe, "Molecular Thermodynamics for Chemical Reactor Design," AIChE J., 20, 20 (1974).

Eckert, C. A., "High Pressure Kinetics in Solution," Annu. Rev. Phys. Chem., 23, 239 (1972).

Eckert, C. A., and B. L. Knutson, "Molecular Charisma in Supercritical Fluids," Fluid Phase Equil., 83, 93 (1993).

Eckert, C. A., D. H. Ziger, K. P. Johnston, and S. Kim, "Solute Par- 
tial Molar Volumes in Supercritical Fluids," J. Phys. Chem., 90, 2738 (1986).

Eckert, C. A., G. W. Leman, and H. H. Yang, "Homogeneous Catalysis for Wet Oxidation: Design and Economic Feasibility of a Mobile Detoxification Unit," $H M C, 20$ (Mar./Apr., 1990).

Economou, I. G., and M. D. Donohue, "Mean Field Calculations of Thermodynamic Properties of Supercritical Fluids," AIChE J., 36, 1920 (1990).

Edwards, T., and S. Zabarnick, "Supercritical Fuel Deposition Mechanisms," Ind. Eng. Chem. Res., 32, 3117 (1993).

Ekart, M. P., K. L. Bennett, S. M. Ekart, G. S. Gurdial, C. L. Liotta, and C. A. Eckert, "Cosolvent Interactions in Supercritical Fluid Solutions," AIChE J., 39, 235 (1993).

Ellington, J. B., and J. F. Brennecke, "Pressure Effect on the Esterification of Phthalic Anhydride in Supercritical $\mathrm{CO}_{2}$, "J. Chem. Soc. Chem. Commun., 1094 (1993).

Ellington, J. B., K. M. Park, and J. F. Brennecke, "Effect of Local Composition Enhancements on the Esterification of Phthalic Anhydride with Methanol in Supercritical Carbon Dioxide," Ind. Eng. Chem. Res., 33, 965 (1994).

Elliott, J. R., Jr., and H. M. Cheung, "Light Scattering Study of Polymer Network Formation in a Supercritical Diluent," Supercritical Fluid Engineering Science, ACS Symp. Ser., No. 514, p. 271 (1993).

Erickson, J. C., P. Schyns, and C. L. Cooney, "Effect of Pressure on an Enzymatic Reaction in a Supercritical Fluid," AIChE J., 36, 299 (1990).

Faeth, G. M., "Current Status of Droplet and Liquid Combustion," Prog. Energy Combust. Sci., 3, 191 (1977).

Fan, L., K. Yokota, and K. Fujimoto, "Supercritical Phase FischerTropsch Synthesis: Catalyst Pore-Size Effect," AIChE J., 38, 1639 (1992).

Fan, L., K. Yokota, and K. Fujimoto, "Supercritical Phase FischerTropsch Synthesis Reaction on Ruthenium Catalyst and its Transfer Phenomena," Proc. Int. Conf. Petrol. Refining Petrochem. Process, 2, 702 (1991).

Flarsheim, W. M., A. J. Bard, and K. P. Johnston, "Pronounced Pressure Effects on Reversible Electrode Reactions in Supercritical Water," J. Phys. Chem., 93, 4234 (1989).

Flarsheim, W. M., Y. Tsou, I. Trachtenberg, K. P. Johnston, and A. J. Bard, "Electrochemistry in Near-Critical and Supercritical Fluids: 3. Studies of $\mathrm{Br}^{-}, \mathrm{I}^{-}$, and Hydroquinone in Aqueous Solutions," J. Phys. Chem., 90, 3857 (1986).

Franck, E. U., "High Pressure Combustion and Flames in Supercritical Water," Proc. Int. Symp. Supercritical Fluids, p. 91 (1991a).

Franck, E. U., "Combustion and Flames in Supercritical Fluids to 2000 bar," Recent Trends in High Pressure Research, Proc. 13th AIRAPT Int. Conf.; High Pressure Science \& Technology, A. K. Singh, ed., Oxford, p. 501 (1991b).

Funazukuri, T., S. Yokoi, and N. Wakao, "Supercritical Fluids Extraction of Chinese Maoming Oil Shale with Water and Toluene," Fuel, 67, 10 (1988).

Funazukuri, T., N. Wakao, and J. M. Smith, "Liquefaction of Lignin Sulphonate with Subcritical and Supercritical Water," Fuel, 69, 349 (1990).

Gabbito, J., S. Hu, B. J. McCoy, and J. M. Smith, "Supercritical Catalytic Dehydrogenation of Toluene," AIChE J., 34, 1225 (1988).

Gaffney, A. M., and J. A. Sofranko, "Selective Oxidation of Propylene to Propylene Glycol in Supercritical Media," ACS Div. Pet. Chem. Prep., 37, 1273 (1992).

Ganapathy, S., T. W. Randolph, and J. A. O'Brien, "Probing the Limit for Bimolecular Reactions in Supercritical Fluids: A Brownian Dynamics and Molecular Dynamics Study," AIChE Meeting, St. Louis (1993).

Gangoli, N., and G. Thodos, "Liquid Fuels and Chemical Feedstocks from Coal by Supercritical Gas Extraction," Ind. Eng. Chem. Prod. Res. Dev., 16, 209 (1977).

Gehrke, Ch., J. Schroeder, D. Schwarzer, J. Troe, and F. Voß, “Photoisomerization of Diphenylbutadiene in Low-Viscosity Nonpolar Solvents: Experimental Manifestations of Multidimensional Kramers Behavior and Cluster Effects," J. Chem. Phys., 92, 4805 (1990).

Ginosar, D. M., and S. Subramaniam, "Coking and Activity of a Reforming Catalyst in Near-critical and Dense Supercritical Reaction Mixtures," Catalyst Deactivation: Proc. Int. Symp. on Catalyst Deac- tivation, B. Delmon and G. F. Froment, eds., Elsevier, Amsterdam (1994).

Gopalan, S., and P. E. Savage, "Supercritical Water Oxidation of Substituted Phenols," AIChE Meeting, St. Louis (1993).

Goto, M., J. M. Smith, and B. J. McCoy, "Kinetics and Mass Transfer for Supercritical Fluid Extraction of Wood," Ind. Eng. Chem. Res., 29, 282 (1990a).

Goto, M., J. O. Hortascu, and B. J. McCoy, "Supercritical Thermal Decomposition of Cellulose: Experiments and Modeling," Ind. Eng. Chem. Res., 29, 1091 (1990b).

Greer, S. C., "Thermal and Chemical Relaxations Near the Critical Point of Chlorine," Phys. Rev. A, 31, 3240 (1985).

Guan, Z., J. R. Combes, Y. Z. Menceloglu, and J. M. DeSimone, "Homogeneous Free Radical Polymerizations in Supercritical Carbon Dioxide: 2. Thermal Decomposition of 2,2'-Azobis(isobutyronitrile)," Macromolecules, 26, 2663 (1993).

Gupta, R. B., J. R. Combes, and K. P. Johnston, "Solvent Effect on Hydrogen Bonding in Supercritical Fluids,"J. Phys. Chem., 97, 707 (1993).

György, I., and G. R. Freeman, "Ionization of Methane and Ethane by X-Rays: Effect of Fluid Density on Energy Absorption (Dosimetry)," J. Appl. Phys., 60, 3188 (1986).

Hamman, S. D., in High Pressure Physics and Chemistry, Vol. 2, R. S. Bradley, ed., Academic Press, London, p. 131 (1963).

Hammond, D. A., M. Karel, A. M. Klibonov, and V. J. Krukonis, "Enzymatic Reactions in Supercritical Gases," Appl. Biochem. Biotech., 11, 393 (1985).

Harradine, D. M., S. J. Buelow, P. C. Dell'orco, R. B. Dyer, B. R. Foy, J. M. Robinson, J. A. Sanchez, T. Sportarelli, and J. D. Wander, "Oxidation Chemistry of Energetic Materials in Supercritical Water," Haz. Waste Haz. Mat., 10, 233 (1993).

Harrison, W. E., III, and D. Hardy, co-chairs, "Structure of Jet Fuels III," ACS Div. Pet. Chem. Prep., 37(2), 371 (1992).

Helling, R. K., and J. W. Tester, "Oxidation of Simple Compounds and Mixtures in Supercritical Water: Carbon Monoxide, Ammonia, and Ethanol," Environ. Sci. Tech., 22, 1319 (1988).

Helling, R. K., and J. W. Tester, "Oxidation Kinetics of Carbon Monoxide in Supercritical Water," Energy Fuels, 1, 417 (1987).

Herrick, D. E., G. D. Holder, Y. T. Shah, and J. Bruggeman, "Acceleration of Chlorination of Alumina Using Supercritical $\mathrm{CCl}_{4}$," AIChE J., 34, 669 (1988).

Hirano, S., T. Yogo, K. Kikuta, and M. Fukuda, "Synthesis of Nickel-zinc Ferrite-dispersed Carbon Composite Particles from Organometallic Compounds," Ceramic Powder Science IV, S. Hirano, G. L. Messing, and H. Hausner, eds., Amer. Cer. Soc., p. 33 (1991).

Hirth, Th., and E. U. Franck, "Oxidation and Hydrothermolysis of Hydrocarbons in Supercritical Water at High Pressures," Ber. Bunsenges. Phys. Chem., 97, 1091 (1993).

Holgate, H. R., and J. W. Tester, "Fundamental Kinetics and Mechanisms of Hydrogen Oxidation in Supercritical Water," Comb. Sci. Tech., 88, 369 (1993).

Holgate, H. R., P. A. Webley, and J. W. Tester, "Carbon Monoxide Oxidation in Supercritical Water: The Effects of Heat Transfer and the Water-Gas Shift Reaction on Observed Kinetics," Energy \& Fuels, 6, 586 (1992).

Holgate, H. R., and J. W. Tester, "Oxidation of Hydrogen and Carbon Monoxide in Sub- and Supercritical Water: Reaction Kinetics, Pathways, and Water-Density Effects: 1. Experimental Results," $J$. Phys. Chem., 98, 800 (1994a).

Holgate, H. R., and J. W. Tester, "Oxidation of Hydrogen and Carbon Monoxide in Sub- and Supercritical Water: Reaction Kinetics, Pathways, and Water-Density Effects: 2. Elementary Reaction Modeling," J. Phys. Chem., 98, 810 (1994b).

Hong, G. T., "Process for Oxidation of Materials in Water at Supercritical Temperatures," Int. Patent Appl. WO 91/11394 (1991).

Horiuchi, A., H. T. Fish, and M. A. Mikita, "Amphoteric Reactions of Supercritical Water with Coal Models," ACS Div. Fuel Chem. Prep., 33, 292 (1988).

Houser, T. J., C. Tsao, J. E. Dyla, M. K. Van Atten, and M. E. McCarville, "The Reactivity of Tetrahydroquinoline, Benzylamine and Bibenzyl with Supercritical Water," Fuel, 68, 323 (1989).

Houser, T. J., D. M. Tiffany, Z. Li, M. E. McCarville, and M. E. Houghton, "Reactivity of Some Organic Compounds with Supercritical Water," Fuel, 65, 827 (1986). 
Houser, T. J., Y. Zhou, C. Tsao, and X. Liu, "Removal of Heteroatoms from Organic Compounds by Supercritical Water," in Supercritical Fluid Engineering Science: Fundamentals and Applications, E. Kiran and J. F. Brennecke, eds., ACS Symp. Ser., No. 514, p. 327 (1993).

Howdle, S. M., and M. Poliakoff, "Organometallic Photochemistry in Supercritical Fluids: The Reaction of $\mathrm{H}_{2}$ with $\left[\eta^{5}-\mathrm{C}_{5} \mathrm{H}_{5} \mathrm{M}(\mathrm{CO})_{3}\right]$ $(\mathrm{M}=\mathrm{Re}$ and $\mathrm{Mn})$ and the Formation of a 'Non-classical' Dihydrogen Complex of Manganese(I)," J. Chem. Soc., Chem. Commun., 1099 (1989).

Howdle, S. M., P. Grebenik, R. N. Perutz, and M. Poliakoff, "The Synthesis and Spectroscopic Identification of $\left[\eta^{5}-\mathrm{C}_{5} \mathrm{H}_{5} \mathrm{Re}\left(\mathrm{N}_{2}\right)_{3}\right]$ and $\left[\eta^{5}-\mathrm{C}_{5} \mathrm{H}_{5} \operatorname{Re}(\mathrm{CO})\left(\mathrm{N}_{2}\right)_{2}\right]$ in Supercritical Xenon at Room Temperature and in $\mathrm{N}_{2}$ Matrices at 20K,"J. Chem. Soc., Chem. Commun., 1517 (1989).

Howdle, S. M., M. A. Healy, and M. Poliakoff, "Organometallic Chemistry in Supercritical Fluids: The Generation and Detection of Dinitrogen and Nonclassical Dihydrogen Complexes of Group 6, 7, and 8 Transition Metals at Room Temperature," J. ACS, 112, 4804 (1990).

Howdle, S. M., M. Jobling, and M. Poliakoff, "Spectroscopic Investigations of Organometallic Photochemistry in Supercritical Fluids," in Supercritical Fluid Technology: Theoretical and Applied Approaches to Analytical Chemistry, F. V. Bright and M. E. P. McNally, eds., ACS Symp. Ser., No. 488 (1992).

Hrnjez, B. J., A. J. Mehta, M. A. Fox, and K. P. Johnston, "Photodimerization of Isophorone in Supercritical Trifluoromethane and Carbon Dioxide," J. Amer. Chem. Soc., 111, 2662 (1989).

Hseih, K. C., J. S. Shuen, and V. Yang, "Droplet Vaporization in High-Pressure Environments 1: Near Critical Conditions," Combust. Sci. Technol., 76, 111 (1991).

Huang, C., "Apparatus and Method for Supercritical Water Oxidation," U.S. Patent 5,100,560 (1992).

Huang, C., H. E. Barner, W. R. Killilea, and G. T. Hong, "Method for Supercritical Water Oxidation," Int. Patent Appl. WO 92/21621 (1992).

Huppert, G. L., B. C. Wu, S. H. Townsend, M. T. Klein, and S. C. Paspek, "Hydrolysis in Supercritical Water: Identification and Implications of a Polar Transition State," Ind. Eng. Chem. Res., 28, 161 (1989).

Ikushima, Y., S. Ito, T. Asano, T. Yokoyama, N. Saito, K. Hatakeda, and T. Goto, "A Diels-Alder Reaction in Supercritical Carbon Dioxide Medium," J. Chem. Eng. Japan, 23, 96 (1990).

Ikushima, Y., N. Saito, and M. Arai, "High Pressure Fourier Transform Infrared Spectroscopy Study of Diels-Alder Reaction of Isoprene and Maleic Anhydride in Supercritical Carbon Dioxide,' Bull. Chem. Soc. Japan, 64, 282 (1991).

Ikushima, Y., N. Saito, and M. Arai, "Supercritical Carbon Dioxide as a Reaction Medium: Examination of Its Solvent Effects in the Near-Critical Region," J. Phys. Chem., 96, 2293 (1992).

Ikushima, Y., N. Saito, T. Yokoyama, K. Hatakeda, S. Ito, M. Arai, and H. W. Blanch, "Solvent Effects on an Enzymatic Ester Synthesis in Supercritical Carbon Dioxide," Chem. Lett., 109 (1993).

Issacs, N., and N. Keating, "The Rates of Diels-Alder Reaction in Liquid and Supercritical Carbon Dioxide,"J. Chem. Soc., Chem. Commun., 876 (1992).

Jessop, P. G., T. Ikariya, and R. Noyori, "Homogeneous Catalytic Hydrogenation of Supercritical Carbon Dioxide," Nature, 368, 231 (1994).

Jin, L., Z. Ding, and M. A. Abraham, "Catalytic Supercritical Water Oxidation of 1,4-Dichlorobenzene," Chem. Eng. Sci., 47, 2659 (1992).

Jin, L., Y. T. Shah, and M. A. Abraham, "The Effect of Supercritical Water on the Catalytic Oxidation of 1,4-Dichlorobenzene," J. Supercrit. Fluids, 3, 233 (1990).

Jobling, M., S. M. Howdle, M. A. Healy, and M. Poliakoff, "Photochemical Activation of $\mathrm{C}-\mathrm{H}$ Bonds in Supercritical Fluids: The Dramatic Effect of Dihydrogen on the Activation of Ethane by $\left[\eta^{5}-\mathrm{C}_{5} \mathrm{Me}_{5} \operatorname{Ir}(\mathrm{CO})_{2}\right]$," J. Chem. Soc., Chem. Commun., 1287 (1990a).

Jobling, M., S. M. Howdle, and M. Poliakoff, "Supercritical Fluids in Organometallics Chemistry: IR Evidence for the Photochemical $\mathrm{C}-\mathrm{H}$ Activation of High Density Polyethylene by $\left[\eta^{5}\right.$. $\mathrm{C}_{5} \mathrm{Me}_{5} \operatorname{Ir}(\mathrm{CO})_{2}$ ]," J. Chem. Soc., Chem. Commun., 1762 (1990b).
Johnston, J. B., R. E. Hannah, V. L. Cunningham, B. P. Daggy, F. J. Sturm, and R. M. Kelly, "Destruction of Pharmaceutical and Biopharmaceutical Wastes by the MODAR Supercritical Water Oxidation Process," Bio/Technology, 6, 1423 (1988).

Johnston, K. P., and C. Haynes, "Extreme Solvent Effects on Reaction Rate Constants at Supercritical Fluid Conditions," AlChE J., 33, 2017 (1987).

Johnston, K. P., S. Kim, and J. Combes, "Spectroscopic Determination of Solvent Strength and Structure in Supercritical Fluid Mixtures: A Review," Supercritical Fluid Science and Technology, K. P. Johnston and J. M. L. Penninger, eds., ACS Symp. Ser., 406, p. 52 (1989).

Kadota, T., and H. Hiroyasu, "Combustion of a Fuel Droplet in Supercritical Gaseous Environments," Symp. (Int.) on Combust., The Combust. Inst., p. 275 (1981).

Kamat, S., J. Barrera, E. J. Beckman, and A. J. Russell, "Biocatalytic Synthesis of Acrylates in Organic Solvents and Supercritical Fluids: I. Optimization of Enzyme Environment," Biotech. Bioeng., 40, 158 (1992).

Kamat, S. V., B. Iwaskewycz, E. J. Beckman, and A. J. Russell, "Biocatalytic Synthesis of Acrylates in Supercritical Fluids: Tuning Enzyme Activity by Changing Pressure," Proc. Nat. Acad. Sci. U.S.A., 90, 2940 (1993).

Kamihira, M., M. Taniguchi, and T. Kobayashi, "Sterilization of Microorganisms with Supercritical Carbon Dioxide," Agric. Biol. Chem., 51, 407 (1987a).

Kamihira, M., M. Taniguchi, and T. Kobayashi, "Synthesis of Aspartame Precursors by Enzymatic Reaction in Supercritical Carbon Dioxide," Agric. Biol. Chem., 51, 3427 (1987b).

Kasche, V., R. Schlothauer, and G. Brunner, "Enzyme Denaturation in Supercritical $\mathrm{CO}_{2}$ : Stabilizing Effect of S-S Bonds During the Depressurization Step," Biotech. Lett., 10, 569 (1988).

Kazarian, S. G., R. B. Gupta, M. J. Clarke, K. P. Johnston, and M. Poliakoff, "How is Hydrogen Bonding Influenced by Solvent Density? The Spectroscopic Study and Modeling of the Interaction between a Proton Donor and Acceptor from the Gas Phase to Supercritical Fluid States," J. ACS., 115, 11099 (1993).

Kershaw, J. R., "Supercritical Fluids in Coal Processing," J. Supercrit. Fluids, 2, 35 (1989).

Killilea, W. R., K. C. Swallow, and G. T. Hong, "The Fate of Nitrogen in Supercritical Water Oxidation," J. Supercrit. Fluids, 5, 72 (1992).

Kim, S., and K. P. Johnston, "Effects of Supercritical Solvents on the Rates of Homogeneous Chemical Reactions," Supercritical Fluids, T. G. Squires and M. E. Paulaitis, eds., ACS Symp. Ser., 329, p. 42 (1987a).

Kim, S., and K. P. Johnston, "Molecular Interactions in Dilute Supercritical Fluid Solutions," Ind. Eng. Chem. Res., 26, 1206 (1987b). Kim, S., and K. P. Johnston, "Adjustment of the Selectivity of a Diels-Alder Reaction Network using Supercritical Fluids," Chem. Eng. Commun., 63, 49 (1988).

Kimura, Y., Y. Yoshimura, and M. Nakahara, "Chemical Reaction in Medium Density Fluid. Solvent Density Effects on the Dimerization Equilibrium of 2-methyl-2-nitrosopropane in Carbon Dioxide," J. Chem. Phys., 90, 5679 (1989).

Kimura, Y., and Y. Yoshimura, "Chemical Equilibrium in Fluids from the Gaseous to Liquid States: Solvent Density Dependence of the Dimerization Equilibrium of 2-methyl-2-nitrosopropane in Carbon Dioxide, Chlorotrifluoromethane, and Trifluoromethane," J. Chem. Phys., 96, 3085 (1992a).

Kimura, Y., and Y. Yoshimura, "Chemical Equilibrium in Simple Fluids: Solvent Density Dependence of the Dimerization Equilibrium of 2-methyl-2-nitrosopropane in Argon and Xenon," J. Chem. Phys., 96, 3824 (1992b).

Kiran, E., "New Supercritical Fluid Processing Concepts Hold Industry Potential," Tappi J., 70, 23 (Nov., 1987a).

Kiran, E., "Supercritical Pulping: A New Concept," Amer. Papermaker, 50, 26 (1987b).

Kiran, E., and V. P. Saraf, "Polymerization of Styrene in Supercritical n-Butane," J. Supercrit. Fluids, 3, 198 (1990).

Kirkwood, J. G., "Theory of Solutions of Molecules Containing Widely Separated Charges with Special Application to Zwitterions," J. Chem. Phys., 2, 351 (1934).

Klein, M. T., Y. G. Mentha, and L. A. Torry, "Decoupling Substituent and Solvent Effects during Hydrolysis of Substituted 
Anisoles in Supercritical Water," Ind. Eng. Chem. Res., 31, 182 (1992).

Klein, M. T., L. A. Torry, B. C. Wu, and S. H. Townsend, "Hydrolysis in Supercritical Water: Solvent Effects as a Probe of the Reaction Mechanism," J. Supercrit. Fluids, 3, 222 (1990).

Knopf, F. C., T.-H. Pang, and K. M. Dooley, "Catalyzed Reactions of Alkylaromatic Hydrocarbons Dissolved in Supercritical Fluids," ACS Div. Fuel Chem. Prep., 32, 359 (1987).

Kodra, D., and V. Balakotaiah, "Modeling of Supercritical Oxidation of Aqueous Wastes in a Deep-Well Reactor," AIChE J., 38, 988 (1992).

Köll, P., B. Brönstrup, and J. O. Metzger, Holzforschung, 33, 112 (1979).

Köll, P., and J. O. Metzger, "Thermal Degradation of Cellulose and Chitin in Supercritical Acetone," Angew. Chem. Int. Ed. Eng., 17, 754 (1978).

Köll, P., and J. O. Metzger, "Liquefaction of Biomass with Supercritical Fluids in a High Pressure/High Temperature Flow Reactor," Chemical Engineering at Supercritical Fluid Conditions, M. E. Paulaitis et al., eds., Ann Arbor Science, Ann Arbor, MI, p. 499 (1983).

Krichevskii, I, R, Y. V. Tsekhanskaya, and Z. A. Polyakova, "Photodissociation of Chlorine and Recombination of Chlorine Atoms at the Critical Point for Liquid-Gas Equilibrium," Russ. J. Phys. Chem., 40, 715 (1966).

Krichevskii, I. R., Y. V. Tsekhanskaya, and L. N. Rozhnovskaya, "Thermodynamics of Infinitely Dilute Critical Solutions. The Equilibrium $\mathrm{N}_{2} \mathrm{O}_{4}=2 \mathrm{NO}_{2}$ in Solution in Carbon Dioxide Near its Critical Point," Russ. J. Phys. Chem., 43, 1393 (1969).

Krukonis, V. J., and D. A. Hammond, "On the Solubility of Disodium P-Nitrophenyl Phosphate in Supercritical Carbon Dioxide," Biotech. Lett., 10, 837 (1988).

Kuang-zong, Q., W. Ren-an, and J. Sheng-sheng, "Chemical Structure Investigation of Maoning Oil Shale Kerogen by Supercritical Gas Extraction," Energy Sources, 7(3), 237 (1984).

Kumar, S. K., R. C. Reid, and U. W. Suter, "Precipitation Polymerization in Supercritical Fluids," Poly. Prep., 27(2), 224 (1986).

Kumar, S. K., and U. W. Suter, "Precipitation Polymerization of Styrene in Supercritical Ethane," Poly. Prep., 28(2), 286 (1987).

Kuznetsov, P. N., V. I. Sharypov, N. G. Beregovtsova, A. I. Rubaylo, and E. D. Korniyets, "Properties of Kansk-Atchinsk Lignite During Liquefaction in Lower Alcohols," Fuels, 69, 911 (1990).

Labrecque, R., S. Kaliaguine, and J. L. Grandmaison, "Supercritical Gas Extraction of Wood with Methanol," Ind. Eng. Chem. Prod. Res. Dev., 23, 177 (1984).

Labrecque, R., S. Kaliaguine, and J. L. Grandmaison, "Supercritical Pyrolysis of Wood," Proc. Bioenergy R\&D Seminar, p. 307 (1982).

Laudise, R. A., "Hydrothermal Synthesis of Crystals," Chem. Eng. News, Sept. 28, p. 30 (1987).

Lawson, J. R., and M. T. Klein, "Influence of Water on Guaiacol Pyrolysis," Ind. Eng. Chem. Fundam., 24, 203 (1985).

Lee, C., and K. L. Hoy, "Supercritical Fluid Based Chemical Reaction Suppression System," Eur. Patent Appl. EP506041A2 (1992).

Lee, D.-S., and E. F. Gloyna, "Hydrolysis and Oxidation of Acetamide in Supercritical Water," Environ. Sci. Tech., 26, 1587 (1992).

Lee, D.-S., E. F. Gloyna, and L. Li, "Efficiency of $\mathrm{H}_{2} \mathrm{O}_{2}$ and $\mathrm{O}_{2}$ in Supercritical Water Oxidation of 2,4-Dichlorophenol and Acetic Acid," J. Supercrit. Fluids, 3, 249 (1990).

Li, R., P. E. Savage, and D. Szmukler, "2-Chlorophenol Oxidation in Supercritical Water: Global Kinetics and Reaction Products," AIChE J., 39, 178 (1993).

Li, R., T. D. Thornton, and P. E. Savage, "Kinetics of $\mathrm{CO}_{2}$ Formation from the Oxidation of Phenols in Supercritical Water," Env. Sci. Tech., 26, 2388 (1992).

Li, L., and N. O. Egiebor, "Oxygen Removal from Coal during Supercritical Water and Toluene Extraction," Energy \& Fuels, 6, 35 (1992).

Li, L., and E. Kiran, "Interaction of Supercritical Fluids with Lignocellulosic Materials," Ind. Eng. Chem. Res., 27, 1301 (1988).

Li, L., and E. Kiran, "Kinetic Model for Supercritical Delignification of Wood," in Supercritical Fluid Science and Technology, K. P. Johnston and J. M. L. Penninger, eds., ACS Symp. Ser., No, 406, p. 317 (1989a).

Li, L., and E. Kiran, "Delignification of Red Spruce by Supercritical
Methylamine and Methylamine-Nitrous Oxide Mixtures," Tappi J., 72, $183(1989 \mathrm{~b})$.

Li, L., P. Chen, and E. F. Gloyna, "Generalized Kinetic Model for Wet Oxidation of Organic Compounds," AIChE J., 37, 1687 (1991).

Li, L., E. F. Gloyna, and J. E. Sawicki, "Treatability of DNT Process Wastewater by Supercritical Water Oxidation,"Water Env. Res., 65, 250 (1993a).

Li, L., P. Chen, and E. F. Gloyna, "Kinetic Model for Wet Oxidation of Organic Compounds in Subcritical and Supercritical Water," in Supercritical Fluid Engineering Science, E. Kiran and J. Brennecke, eds., ACS Symp. Ser. No. 514, p. 305 (1993b).

Li, Z., and T. J. Houser, "Kinetics of the Catalyzed Supercritical Water-Quinoline Reaction," Ind. Eng. Chem. Res., 31, 2456 (1992).

Lin, H-M., Z. Yang, and L.-F. Chen, "Inactivation of Saccharomyces Cerevisiae by Supercritical and Subcritical Carbon Dioxide," Biotech. Prog., 8, 458 (1992).

Lira, C. T., and P. J. McCrackin, "Conversion of Lactic Acid to Acrylic Acid in Near-Critical Water," Ind. Eng. Chem. Res., 32, 2608 (1993).

Liu, Q., J. Wang, and A. H. Zewail, "Femtosecond Dynamics of Dissociation and Recombination in Solvent Cages," Nature, 364, 427 (1993).

Low, J. Y., "Hydrotreating in Supercritical Media," ACS Div. Fuel Chem. Prep., 30, 161 (1985).

Maddocks, R. R., J. Gibson, and D. F. Williams, "Supercritical Extraction of Coal," Chem. Eng. Prog., 75, 49 (1979).

Manos, G., and H. Hofmann, "Coke Removal from a Zeolite Catalyst by Supercritical Fluids," Chem. Eng. Technol., 14, 73 (1991).

Margolis, S. B., and F. A. Williams, "Flame Propagation in Solids and High-density Fluids with Arrhenius Reactant Diffusion," Combust. Flame, 83, 390 (1991).

Margolis, S. B., and S. C. Johnston, "Multiplicity and Stability of Supercritical Combustion in a Nonadiabatic Tubular Reactor," Comb. Sci. Tech., 65, 103 (1989).

Martin, T. G., and D. F. Williams, "The Chemical Nature of Supercritical Gas Extracts from Low Rank U.K. Coals," Phil. Trans. R. Soc. London, A300(1453), 183 (1981).

Martins, J. F., S. B. Barreiros, E. G. Azevedo, and M. Nunes da Ponte, "Lipase Catalyzed Esterification of Glycidol in Near Critical Carbon Dioxide," Proc. Int. Symp. Supercritical Fluids, p. 406 (1991).

Marty, A., W. Chulalaksananukul, J. S. Condoret, R. M. Willemot, and G. Durand, "Comparison of Lipase-Catalysed Esterification in Supercritical Carbon Dioxide and in $n$-Hexane," Biotech. Lett., 12, 11 (1990).

Marty, A., W. Chulalaksananukul, J. S. Condoret, and D. Combes, "Transesterification and Esterification in Supercritical Carbon Dioxide," High Pressure Biotechnol., 224, 461 (1992a).

Marty, A., W. Chulalaksananukul, R. M. Willemot, and J. S. Condoret, "Kinetics of Lipase-Catalyzed Esterification in Supercritical $\mathrm{CO}_{2}$," Biotech. Bioeng., 39, 273 (1992b).

Makabe, M., and K. Ouchi, "Effect of Pressure and Temperature on the Reaction of Coal with Alcohol-Alkali," Fuel, 60, 327 (1981).

Masten, D. A., B. R. Foy, D. M. Harradine, and R. B. Dyer, "In Situ Raman Spectroscopy of Reactions in Supercritical Water," J. Phys. Chem., 97, 8557 (1993).

McCoy, B. J., and B. Subramaniam, "Continuous-Mixture Kinetics of Coke Formation from Olefinic Oligomers," $A I C h E J$, 40, 131 (1994).

McDonald, A. C., F. F. Fan, and A. J. Bard, "Electrochemistry in Near-Critical and Supercritical Fluids: 2. Water-Experimental Techniques and the Copper (II) System," J. Phys. Chem., 90, 196 (1986)

McDonald, E. C., J. Howard, and B. Bennett, "Chemicals from Forest Products by Supercritical Fluid Extraction," Fluid Phase Equil., 10, 337 (1983).

McFarland, R. D., G. Brewer, and C. K. Rofer, "Design and Operation Parameters of Transportable Supercritical Water Oxidation Waste Destruction Unit," Los Alamos National Lab. Rep. LA12216-MS (1991).

McHugh, M. A., and V. J. Krukonis, "Supercritical Fluids," in Ency. clopedia of Polymer Science and Engineering, Vol. 16, p. 368 (1989).

McHugh, M., and V. Krukonis, Supercritical Fluid Extraction, 1st ed., Butterworths, Boston (1986) 
McHugh, M., and V. Krukonis, Supercritical Fluid Extraction, 2nd ed., Butterworth-Heinemann, Boston (1994).

McHugh, M., and M. E. Paulaitis, "Solid Solubilities of Naphthalene and Biphenyl in Supercritical Carbon Dioxide," J. Chem. Eng. Data, 25, 326 (1980).

McRae, E. G., "Theory of Solvent Effects on Molecular Electronic Spectra. Frequency Shifts," J. Phys. Chem., 61, 562 (1957).

Metzger, J. O., J. Hartmanns, D. Malwitz, and P. Köll, "Thermal Organic Reactions in Supercritical Fluids," in Chemical Engineering at Supercritical Fluid Conditions, M. E. Paulaitis et al., eds., Ann Arbor Science, Ann Arbor, MI, p. 515 (1983).

Mikita, M. A., and H. T. Fish, "Reactions of Coal and Coal Model Compounds with Supercritical Water," ACS Div. Fuel Chem. Prep., 31, 56 (1986).

Miller, D. A., H. W. Blanch, and J. M. Prausnitz, "Enzyme-Catalyzed Interesterification of Triglycerides in Supercritical Carbon Dioxide," Ind. Eng. Chem. Res., 30, 939 (1991).

Miller, D. A., H. W. Blanch, and J. M. Prausnitz, "Enzymatic Interesterification of Triglycerides in Supercritical Carbon Dioxide," Ann. N.Y. Acad. Sci., Enzyme Engineering 10, p. 534 (1990).

Milner, S. T., and P. C. Martin, "Critical Slowing of Chemical Reactions," Phys. Rev. A, 33, 1996 (1986).

Minier, L. M., K. R. Brower, and J. C. Oxley, "Role of Intermolecular Reactions in Thermolysis of Aromatic Nitro Compounds in Supercritical Aromatic Solvents," J. Org. Chem., 56, 3306 (1991).

Modell, M., E. F. Kuharich, and M. R. Rooney, "Supercritical Water Oxidation Process and Apparatus of Organics with Inorganics," Int. Patent Appl. WO 93/00304 (1993).

Modell, M., J. Larson, and S. F. Sobczynski, "Supercritical Water Oxidation of Pulp Mill Sludges," Tappi J., 75(6), 195 (1992).

Modell, M., "Processing Methods for the Oxidation of Organics in Supercritical Water,"U.S. Patent 4,543,190 (1985).

Modell, M., "Processing Methods for the Oxidation of Organics in Supercritical Water,'U.S. Patent 4,338,199 (1982).

Modell, M., "Gasification and Liquefaction of Forest Products in Supercritical Water," Fundamentals of Thermochemical Biomass Conversion, Elsevier, Amsterdam, p. 95 (1985).

Modell, M., Standard Handbook of Hazardous Waste Treatment and Disposal, Sec. 8.11, H. M. Freeman, ed., McGraw-Hill, New York (1989).

Mok, W. S., M. J. Antal, Jr., and M. Jones, Jr., "Formation of Acrylic Acid from Lactic Acid in Supercritical Water,"J. Org. Chem., 54, 4596 (1989).

Mok, W. S., and M. J. Antal, Jr., "Dilute Acid Hydrolysis of Biopolymers in a Semi-Batch Flow Reactor at Supercritical Pressure," Energy Biomass Wastes, 13, 1329 (1990).

Moore, J. W, and R. G. Pearson, Kinetics and Mechanism, 3rd ed., Wiley, New York (1981).

Morrison, G., "Comment on 'Anomalies in Chemical Equilibria Near Critical Points'," Phys. Rev. A, 30, 644 (1984).

Muchmore, C. B., J. W. Chen, A. C. Kent, and K. E. Tempelmeyer, "Removal of Organic Sulfur from Coal by Reaction with Supercritical Alcohols," Fossil Fuels Utilization: Environmental Concerns, Markuszewski and Blanustein et al., eds., ACS Symp. Ser., No. 319,75 (1986).

Nakagawa, K., "Photoionization Processes in Nonpolar Media: Density Effect," Radiat. Phys. Chem., 37, 643 (1991).

Nakamura, K., "Biochemical Reactions in Supercritical Fluids," Tibtech, 8, 288 (1990).

Nakamura, K., H. Fujii, Y. M. Chi, and T. Yano, "Supercritical Fluid," Ann. N.Y. Acad. Sci., Enzyme Engineering 10, p. 319 (1990).

Nakamura, K., Y. M. Chi, Y. Yamada, and T. Yano, "Lipase Activity and Stability in Supercritical Carbon Dioxide," Chem. Eng. Commun., 45, 207 (1986).

Narayan, R., and M. J. Antal, Jr., "Kinetic Elucidation of the AcidCatalyzed Mechanism of 1-Propanol Dehydration in Supercritical Water," in Supercritical Fluid Science and Technology, K. P. Johnston and J. M. L. Penninger, eds., ACS Symp. Ser., No. 406, 227 (1989).

Narayan, R., and M. J. Antal, Jr., "Influence of Pressure on the Acid-Catalyzed Rate Constant for 1-Propanol Dehydration in Supercritical Water," J. ACS., 112, 1927 (1990).

Niehaus, D., M. Philips, A. Michael, and R. M. Wightman, "Voltammetry of Ferrocene in Supercritical $\mathrm{CO}_{2}$ Containing Water and
Tetrahexylammonium Hexafluorophosphate," J. Phys. Chem., 93, 6232 (1989).

Nishikawa, E., Y. Shimada, T. Funazukuri, and N. Wakao, "Reactions between Germanium Oxide and Platinum in the Environment of Super/Subcritical Water," Kagaku Kogaku Ronbunshu, 17, 1229 (1991).

Nishikawa, E., and N. Wakao, "Reactions between Germanium and Platinum in Sub/Supercritical Water," Can. J. Chem. Eng., 70, 608 (1992).

Nywlt, M., and S. Peter, "Improvement of Coal Gasification," Sci. Technol., 45, 349 (1992).

O'Brien, J. A., T. W. Randolph, C. Carlier, and S. Ganapathy, "Quasicritical Behavior of Dense-Gas Solvent-Solute Clusters at NearInfinite Dilution," AIChE J., 39, 1061 (1993).

Occhiogrosso, R. N., and M. A. McHugh, "Critical Mixture Oxidation of Cumene," Chem. Eng. Sci., 42, 2478 (1987).

Okada, T., Y. Kobayashi, H. Yamasa, and N. Mataga, "Intramolecular Exciplex Fluorescence in Supercritical Fluids," Chem. Phys. Lett., 128, 583 (1986).

Olcay, A., T. Tugrul, and A. Calimli, "The Supercritical Gas Extraction of Lignites and Wood," in Chemical Engineering at Supercritical Fluid Conditions, M. E. Paulaitis, J. M. L. Penninger, R. Gray, and P. Davidson, eds., Ann Arbor Science, Ann Arbor, MI, p. 409 (1983).

Olesik, S. V., S. B. French, and M. Novotny, "Reaction Monitoring in Supercritical Fluids by Flow Injection Analysis with Fourier Transform Infrared Spectroscopic Detection," Anal. Chem., 58, 2256 (1986).

O'Shea, K. E., K. M. Kirmse, M. A. Fox, and K. P. Johnston, "Polar and Hydrogen Bonding Interactions in Supercritical Fluids. Effects of the Tautomeric Equilibrium of 4-(Phenylazo)-1-naphthol," $J$. Phys. Chem., 95, 7863 (1991a).

O'Shea, K. E., J. R. Combes, M. A. Fox, and K. P. Johnston, "Photolysis of Dibenzylketones in Supercritical Ethane and Carbon Dioxide," Photochem. Photobiol., 54, 571 (1991b).

Otto, B., J. Schroeder, and J. Troe, "Photolytic Cage Effect and Atom Recombination of lodine in Compressed Gases and Liquids: Experiments and Simple Models," J. Chem. Phys., 81, 202 (1984).

Pasta, P., G. Mazzola, G. Carrea, and S. Riva, "Subtilisin-Catalyzed Transesterification in Supercritical Carbon Dioxide," Biotech. Lett., 11, 643 (1989).

Paulaitis, M. E., and G. C. Alexander, "Reactions in Supercritical Fluids. A Case Study of the Thermodynamic Solvent Effects on a Diels-Alder Reaction in Supercritical Carbon Dioxide," Pure Appl. Chem., 59, 61 (1987).

Peck, D. G., A. J. Mehta, and K. P. Johnston, "Pressure Tuning of Chemical Reaction Equilibria in Supercritical Fluids," $J$. Phys. Chem., 93, 4297 (1989).

Penninger, J. M. L., "Extraction of Oil from Wyoming Coal with Aqueous Solvents at Elevated Pressure," in Supercritical Fluid Technology, J. M. L. Penninger, M. Radosz, M. A. McHugh, and V. J. Krukonis, eds., Elsevier, Amsterdam (1985).

Penninger, J. M. L., "Reactions in Di-n-butylphthalate at Near-Critical Temperature and Pressure," Fuel, 67, 490 (1988).

Penninger, J. M. L., "Selectivity Effects in Aqueous Supercritical Fluid Extraction of Subbituminous Coal," Fuel, 68, 983 (1989).

Penninger, J. M. L., and J. M. M. Kolmschate, "Chemistry of Methoxynaphthalene in Supercritical Water," Supercritical Fluid Science and Technology, K. P. Johnston and J. M. L. Penninger, eds., ACS Symp. Ser., No. 406, 242 (1989).

Perrut, M., "Enzymatic Reactions in Supercritical Carbon Dioxide," High Pressure Biotechnol., 224, 401 (1992).

Petsche, I. B., and P. G. Debenedetti, "Solute-Solvent Interactions in Infinitely Dilute Supercritical Mixtures: A Molecular Dynamics Investigation," J. Chem. Phys., 91, 7075 (1989).

Philips, M. E., M. R. Deakin, M. V. Novotny, and R. M. Wightman, "Effect of Added Water on Voltammetry in Near-Critical Carbon Dioxide," J. Phys. Chem., 91, 3934 (1987).

Poirer, M. G., A. Ahmed, J. Grandmaison, and S. C. F. Kaliaguine, "Supercritical Gas Extraction of Wood with Methanol in Tubular Reactor," Ind. Eng. Chem. Res., 26, 1738 (1987).

Poliakoff, M., S. M. Howdle, M. A. Healy, and J. M. Whalley, "Photochemistry and Spectroscopy in Supercritical Fluids near Room Temperature," Proc. Int. Symp. Supercritical Fluids, p. 967 (1988).

Poliakoff, M., S. M. Howdle, M. Jobling, and M. W. George, 
"Organometallic Chemistry in Supercritical Fluids," Proc. 2nd Int. Symp. Supercritical Fluids, p. 189 (1991).

Pommier, C., K. Chhor, J. F. Bocquet, and M. Barj, "Reactions in Supercritical Fluids, a New Route for Oxide Ceramic Powder Elaboration. Synthesis of the Spinel $\mathrm{MgAl}_{2} \mathrm{O}_{4}$," Mat. Res. Bull., 25, 213 (1990).

Procaccia, I., and M. Gitterman, "Slowing Down of Chemical Reactions near Thermodynamic Critical Points," Phys. Rev. Lett., 46, 1163 (1981).

Procaccia, I., and M. Gitterman, "Dynamical Critical Phenomena in Chemically Reactive Fluid Mixtures," Phys. Rev. A, 25, 1137 (1982).

Procaccia, I., and M. Gitterman, "Anomalies in Chemical Equilibria Near Critical Points," Phys. Rev. A, 27, 555 (1983).

Rabenau, A., "The Role of Hydrothermal Synthesis in Preparative Chemistry," Angew. Chem. Int. Ed. Eng., 24, 1026 (1985).

Ramayya, S., A. Brittain, C. DeAlmeida, W. Mok, and M. J. Antal, Jr.," Acid-Catalyzed Dehydration of Alcohols in Supercritical Water," Fuel, 66, 1364 (1987).

Randolph, T. W., H. W. Blanch, J. M. Prausnitz, and C. R. Wilke, "Enzymatic Catalysis in a Supercritical Fluid," Biotech. Lett., 7, 325 (1985).

Randolph, T. W., D. S. Clark, H. W. Blanch, and J. M. Prausnitz, "Cholesterol Aggregation and Interaction with Cholesterol Oxidase in Supercritical Carbon Dioxide," Proc. Nat. Acad. Sci. U.S.A., 85, 2979 (1988a)

Randolph, T. W., D. S. Clark, H. W. Blanch, and J. M. Prausnitz, "Enzymatic Oxidation of Cholesterol Aggregates in Supercritical Carbon Dioxide," Science, 239, 387 (1988b).

Randolph, T. W., H. W. Blanch, and J. M. Prausnitz, "Enzymatic Oxidation of Cholesterol Aggregates in Supercritical Carbon Dioxide," AIChE J., 34, 1354 (1988c).

Randolph, T. W., H. W. Blanch, and D. S. Clark, "Biocatalysis in Supercritical Fluids," Biocatal. Ind., Chap. 11, J. S. Dordick, ed., Plenum, New York, p. 219 (1991).

Randolph, T. W., and C. Carlier, "Free Radical Reactions in Supercritical Ethane: A Probe of Supercritical Fluid Structure," J. Phys. Chem., 96, 5146 (1992a).

Randolph, T. W., and C. Carlier, "Pressure Control of Reactions in Supercritical Fluids: Thermodynamics and Kinetics," Biocatalysis in Non-Conventional Media, J. Tramper et al., eds., Elsevier, Amsterdam, p. 93 (1992b).

Randolph, T. W., J. A. O'Brien, and S. Ganapathy, "Does Critical Clustering Affect Reaction Rate Constants? Molecular Dynamics Studies in Pure Supercritical Fluids," J. Phys. Chem., 98, 4173 (1994).

Rathke, J. W., R. J. Klingler, and T. R. Krause, "Thermodynamics of the Hydrogenation of Dicobalt Octacarbonyl in Supercritical Carbon Dioxide," Organometallics, 11, 585 (1992).

Rathke, J. W., R. J. Klingler, and T. R. Krause, "Propylene Hydroformylation in Supercritical Carbon Dioxide," Organometallics, 10, 1350 (1991).

Reetz, M. T., W. Könen, and T. Strack, "Supercritical Carbon Dioxide as a Reaction Medium and Reaction Partner," Chimia, 47, 493 (1993).

Reid, R. C., J. M. Prausnitz, and T. K. Sherwood, The Properties of Gases and Liquids, 3rd ed., McGraw-Hill, New York (1977).

Reyes, T., S. Bandyopadhyay, and B. J. McCoy, "Extraction of Lignin from Wood with Supercritical Alcohols,"J. Supercrit. Fluids, 2, 80 (1989).

Rice, S. F., R. R. Steeper, and C. A. LaJeunesse, "Destruction of Representative Navy Wastes Using Supercritical Water Oxidation," Sandia National Lab. Rep. SAND94-8203 (1993).

Roberts, C. B., J. E. Chateauneuf, and J. F. Brennecke, "Unique Pressure Effects on the Absolute Kinetics of Triplet Benzophenone Photoreduction in Supercritical $\mathrm{CO}_{2}, "$ J. ACS, 114, 8455 (1992).

Roberts, C. B., J. Zhang, J. F. Brennecke, and J. E. Chateauneuf, "Laser Flash Photolysis Investigation of Diffusion-Controlled Reactions in Supercritical Fluids," J. Phys. Chem., 97, 5618 (1993a).

Roberts, C. B., J. Zhang, J. E. Chateauneuf, and J. F. Brennecke, "Diffusion-Controlled Reactions in Supercritical $\mathrm{CHF}_{3}$ and $\mathrm{CO}_{2}$ /Acetonitrile Mixtures," J. ACS, 115, 9576 (1993b).

Rofer, C. K., "Supercritical Water Oxidation," Proc. Int. Symp. Chemical Oxidation, Technomic Publishing, Lancaster, PA, p. 278 (1991).
Rofer, C. K., and G. E. Streit, "Kinetics and Mechanisms of Methane Oxidation in Supercritical Water,"Los Alamos National Lab. Rep. LA-11439-MS (1988).

Rofer, C. K., and G. E. Streit, "Phase II Final Report: Oxidation of Hydrocarbons and Organics in Supercritical Water," Los Alamos National Lab. Rep. LA-11700-MS (1989).

Rofer, C. K., S. J. Buelow, R. B. Dyer, and J. D. Wander, "Conversion of Hazardous Materials Using Supercritical Water Oxidation," U.S. Patent 5,133,877 (1992).

Ross, D. S., T. K. Green, and R. Mansani, "Coal Conversion in Supercritical Water Systems," Proc. Int. Coal Conf., Pittsburgh, 10 (1983).

Ross, D. S., "Coal Conversion in Carbon Monoxide-Water Systems," Coal Sci., 3, 301 (1984).

Ross, D. S., G. P. Hum, and T. Miin, "Isotope Effects in Supercritical Water-Kinetic Studies of Coal Liquefaction," ACS Div. Fuel Chem. Prep., 30, 94 (1985).

Ross, D. S., G. P. Hum, T. Miin, T. K. Green, and R. Mansani, "Isotope Effects in Supercritical Water: Kinetic Studies of Coal Liquefaction," in Supercritical Fluids - Chemical and Engineering Principles and Applications, T. G. Squires and M. E. Paulaitis, eds., ACS Symp. Ser. 329, 242 (1987).

Russell, A. J., and E. J. Beckman, "Enzyme Activity in Supercritical Fluids,” Appl. Biochem. Biotech., 31, 197 (1991a).

Russell, A. J., and E. J. Beckman, "Should the High Diffusivity of a Supercritical Fluid Increase the Rate of an Enzyme-Catalyzed Reaction?" Enzyme Microb. Technol., 13, 1007 (1991b).

Russell, A. J., E. J. Beckman, and A. K. Chaudhary, "Studying Enzyme Activity in Supercritical Fluids," Chemtech., 33 (Mar., 1994).

Saim, S., and B. Subramaniam, "Chemical Reaction Equilibrium at Supercritical Conditions," Chem. Eng. Sci., 43, 1837 (1988).

Saim, S., D. M. Ginosar, and B. Subramaniam, "Phase and Reaction Equilibria Considerations and Operation of Supercritical Fluid Reaction Processes," Stpercritical Fluid Science and Technology, K. P. Johnston, ed., ACS Symp. Ser., No. 406, p. 301 (1989).

Saim, S., and B. Subramaniam, "Isomerization of 1-Hexane on $\mathrm{Pt} / \mathrm{y}$ $\mathrm{Al}_{2} \mathrm{O}_{3}$ Catalyst at Subcritical and Supercritical Reaction Conditions: Pressure and Temperature Effects on Catalyst Activity," $J$. Supercrit. Fluids, 3, 214 (1990).

Saim, S., and B. Subramaniam, "Isomerization of 1-Hexene over $\mathrm{Pt} / \gamma-\mathrm{Al}_{2} \mathrm{O}_{3}$ Catalyst: Reaction Mixture Density and Temperature Effects on Catalyst Effectiveness Factor, Coke Laydown, and Catalyst Micromeritics,"J. Catal., 131, 445 (1991).

Sandler, S. I., Chemical and Engineering Thermodynamics, 2nd ed., Wiley, New York (1989).

Savage, P. E., E. E. Brock, and J. Santini, "Reaction Paths and Mechanisms in Supercritical Water," AIChE Meeting, St. Louis, MO (1993)

Savage, P. E., S. Gopalan, and R. Li, "Oxidation of Phenols in Supercritical Water," Chemical Oxidation: Technology for the Nineties, Proc. 3rd Int. Symp. Chem. Oxid., Technomic Publishers, Lancaster, PA, p. 34 (1994a).

Savage, P. E., R. Li, and J. T. Santini, "Methane to Methanol in Supercritical Water,"J. Supercrit. Fluids, 7, 135 (1994b).

Sawicki, J. E., and B. Casas, "Wet Oxidation Systems--Process Concept to Design," Environ. Prog., 12, 275 (1993).

Schilling, W., and E. U. Franck, "Combustion and Diffusion Flames at High Pressures to 2000 bar," Ber. Bunsenges. Phys. Chem., 92, 631 (1988).

Scholsky, K. M., "Polymerization Reactions at High Pressure and Supercritical Conditions," J. Supercrit. Fluids, 6, 103 (1993).

Shanableh, A., and E. F. Gloyna, "Supercritical Water Oxidation-Wastewater and Sludges," Water Sci. Tech., 23, 389 (1991).

Shaw, R. W., T. B. Brill, A. A. Clifford, C. A. Eckert, and E. U. Franck, "Supercritical Water a Medium for Chemistry," Chem. Eng. News, 69(51), 26 (1991).

Shen, X. M., T. W. de Loos, and J. de Swaan Arons, "Ezymatic Reaction in Organic Solvents and Supercritical Gases," Biocatal. Non-Conv. Media, 417 (1992).

Shuen, J. S., V. Yang, and C. C. Hsiao, "Combustion of Liquid-Fuel Droplets in Supercritical Conditions," Combustion Flame, 89, 299 (1992).

Sigman, M. E., and J. E. Leffler, "Supercritical Carbon Dioxide. The Cis to Trans Relaxation and $\pi, \pi^{*}$ Transition of 4-(Diethylamino)4'-nitroazobenzene," J. Org. Chem., 52, 3123 (1987). 
Sigman, M. E., J. T. Barbas, and J. E. Leffler, "Supercritical Carbon Dioxide. 5. Carboxyinversion Reactions of Diacyl Peroxides. Alkyl Group Rearrangement and $\mathrm{CO}_{2}$ Exchange," J. Org. Chem., 52, 1754 (1987).

Silvestri, G., S. Gambino, G. Filardo, C. Cuccia, and E. Guarino, "Electrochemical Processes in Supercritical Phases," Angew. Chem. Int. Ed. Eng., 20, 101 (1981).

Simkovic, I., T. Leesonboon, W. Mok, and M. J. Antal, Jr., "Dehydration of Carbohydrates in Supercritical Water," ACS Div. Fuel Chem. Prep., 32, 129 (1987).

Squires, T. G., C. G. Venier, and T. Aida, "Supercritical Fluid Solvents in Organic Chemistry," Fluid Phase Equilibria, 10, 261 (1983).

Srinivasan, G., and J. R. Elliott, Jr., "Molecular Materials via Polymerization in Supercritical Fluids," Ind. Eng. Chem. Res., 31, 1414 (1992).

Staszak, C. N., K. C. Malinowski, and W. R. Killilea, "The Pilot-Scale Demonstration of the MODAR Oxidation Process for the Destruction of Hazardous Organic Waste Materials," Environ. Prog., 6, 39 (1987)

Steeper, R. R., S. F. Rice, M. S. Brown, and S. C. Johnston, "Methane and Methanol Diffusion Flames in Supercritical Water," J. Supercrit. Fluids, 5, 262 (1992).

Steinfeld, J. I., J. S. Francisco, and W. L. Hase, Chemical Kinetics and Dynamics, Prentice-Hall, Englewood Cliffs, NJ (1989).

Subramaniam, B., and M. A. McHugh, "Reactions in Supercritical Fluids-A Review," Ind. Eng. Chem. Process Des. Dev., 25, 1 (1986).

Subramaniam, B., and B. J. McCoy, "Catalyst Activity Maintenance or Decay: A Model for Formation and Desorption of Coke," Ind. Eng. Chem. Res., 33, 504 (1994).

Sunol, A. K., and G. H. Beyer, "Mechanism of Supercritical Extraction of Coal," Ind. Eng. Chem. Res., 29, 842 (1990).

Sunol, A. K., "Supercritical Fluid-aided Treatment of Porous Materials," Int. Patent Appl. WO 90/02612 (1992).

Suppes, G. J., and M. A. McHugh, "Solvent and Catalytic Metal Effects on the Decomposition of Cumene Hydroperoxide," Ind. Eng. Chem. Res., 28, 1146 (1989).

Suppes, G. J., R. N. Occhiogrosso, and M. A. McHugh, "Oxidation of Cumene in Supercritical Reaction Media," Ind. Eng. Chem. Res., 28, 1152 (1989)

Swallow, K. C., and W. R. Killilea, "Comment on 'Phenol Oxidation in Supercritical Water: Formation of Dibenzofuran, Dibenzo-p-dioxin, and Related Compounds'," Environ. Sci. Tech., 26, 1848 (1992).

Sweeney, P. G., V. Gutenkunst, J. Nowok, and V. I. Stenberg, "Temperature Programmed Aqueous Liquefaction Extended to Supercritical Conditions," ACS Div. Fuel Chem. Prep., 34, 824 (1989).

Takahashi, Y., T. Wydeven, T. Koo, and C. Koo, "Suberitical and Supercritical Water Oxidation of CELSS Model Wastes," Adv. Space Res., 9, 99 (1989).

Taniguchi, M., M. Kamihira, and T. Kobayashi, "Effect of Treatment with Supercritical Carbon Dioxide on Enzymatic Activity," Agric. Biol. Chem., 51(2), 593 (1987).

Tanko, J. M., and J. F. Blackert, "Free-Radical Side-Chain Bromination of Alkylaromatics in Supercritical Carbon Dioxide," Science, 263, 203 (1994).

Terry, R. E., A. Ziad, C. Angelos, and D. L. Whitman, "Polymerization in Supercritical $\mathrm{CO}_{2}$ to Improve $\mathrm{CO}_{2} / \mathrm{Oil}$ Mobility Ratios," Energy Prog., 8, 48 (1988).

Tester, J. W., H. R. Holgate, F. J. Armellini, P. A. Webley, W. R. Killilea, G. T. Hong, and H. E. Barner, "Supercritical Water Oxidation Technology: Process Development and Fundamental Research," in Emerging Technologies in Hazardous Waste Management III, ACS Symp. Ser. 518, p. 35 (1993).

Tester, J. W., P. A. Webley, and H. R. Holgate, "Revised Global Kinetics Measurements of Methanol Oxidation in Supercritical Water," Ind. Eng. Chem. Res., 32, 236 (1992).

Thomason, T. B., G. T. Hong, K. C. Swallow, and W. R. Killilea, "The MODAR Supercritical Water Oxidation Process," in Innovative Hazardous Waste Treatment Technology Series: Thermal Processes, Harry M. Freeman, ed., Technomic Publishing, Lancaster, PA, p. 31 (1990).

Thomason, T. B., and M. Modell, "Supercritical Water Destruction of Aqueous Wastes," Haz. Waste, 1, 453 (1984).

Thornton, T. D., "Phenol Oxidation in Supercritical Water: Reaction
Kinetics, Products, and Pathways," PhD Diss., Univ. of Michigan, Ann Arbor (1991).

Thornton, T. D., and P. E. Savage, "Phenol Oxidation in Supereritical Water," J. Supercrit. Fluids, 3, 240 (1990).

Thornton, T. D., and P. E. Savage, "Phenol Oxidation Pathways in Supercritical Water," Ind. Eng. Chem. Res., 31, 2451 (1992a).

Thornton, T. D., and P. E. Savage, "Kinetics of Phenol Oxidation in Supercritical Water," AIChE J, 38, 321 (1992b).

Thornton, T. D., D. E. LaDue, and P. E. Savage, "Phenol Oxidation in Supercritical Water: Formation of Dibenzofuran, Dibenzo-p-dioxin, and Related Compounds," Environ. Sci. Tech., 25, 1507 (1991).

Tiltscher, W., H. Wolf, and J. Schelchshorn, "A Mild and Effective Method for the Reactivation or Maintenance of Activity of Heterogeneous Catalyst," Angew. Chem., Int. Ed., 20, 892 (1981).

Tiltscher, H., and H. Hofmann, "Trends in High Pressure Chemical Reaction Engineering," Chem. Eng. Sci,, 42, 959 (1987).

Tilscher, H., H. Wolf, and J. Schelchshorn, "Utilization of Supercritical Fluid Solvent Effects in Heterogeneous Catalysis," Ber. Bunsenges. Phys. Chem., 88, 897 (1984).

Timberlake, S. H., G. T. Hong, M. Simson, and M. Modell, "Supercritical Water Oxidation for Wastewater Treatment: Preliminary Study of Urea Destruction," SAE Tech. Paper 820872 (1982).

Titmas, J. A., "Method and Apparatus for Conducting Chemical Reactions at Supercritical Conditions," U.S. Patent 4,594,164 (1986).

Tom, J. W., and P. G. Debenedetti, "Integral Equation Study of Microstructure and Solvation in Model Attractive and Repulsive Supercritical Mixtures," Ind. Eng. Chem. Res., 32, 2118 (1993).

Torry, L. A., R. Kaminsky, M. T. Klein, and M. R. Klotz, "The Effect of Salts on Hydrolysis in Supercritical and Near-Critical Water: Reactivity and Availability," J. Supercrit. Fluids, 5, 163 (1992).

Townsend, S. H., and M. T. Klein, "Dibenzylether as a Probe into the Supercritical Fluid Solvent Extraction of Volatiles from Coal with Water," Fuel, 64, 635 (1985).

Townsend, S. H., M. A. Abraham, G. L. Huppert, M. T. Klein, and S. C. Paspek, "Solvent Effects During Reactions in Supercritical Water," Ind. Eng. Chem. Res., 27, 143 (1988).

Triday, J., and J. M. Smith, "Dynamic Behavior of Supercritical Ex traction of Kerogen from Shale," AIChE J., 34, 658 (1988).

Troe, J., "Elementary Reactions in Compressed Gases and Liquids: From Collisional Energy Transfer to Diffusion Control," J. Phys. Chem., 90, 357 (1986).

Tsao, C. C., Y. Zhou, X. Liu, and T. J. Houser, "Reactions of Supercritical Water with Benzaldehyde, Benzylidenebenzylamine, Benzyl Alcohol, and Benzoic Acid," J. Supercrit. Fluids, 5, 107 (1992).

Tugrul, T., and A. Olcay, "Supercritical Gas Extraction of Two Lignites," Fuel, 57, 415 (1978).

Tyler, A. L., and R. F. Cane, "Pyrolysis of Oil Shale and Related Organic Compounds in Supercritical Steam," Energy Technol. Conf. Proc., 9, 580 (1982)

Umemura, A., "Supercritical Liquid Fuel Combustion," Proc. Int. Symp. on Combustion, The Combust. Inst., p. 463 (1986).

VanEldik, R., T. Asano, and W. J. LeNoble, "Activation and Reaction Volumes in Solution. 2," Chem. Rev., 89, 549 (1989).

Vick Roy, J. R., and A. O. Converse, "Biomass Hydrolysis with Sulfur Dioxide and Water in the Region of the Critical Point," Supercritical Fluid Technology, J. M. L. Penninger, M. Radosz, M. A. McHugh, and V.J. Krukonis, eds., Elsevier, Amsterdam, p. 397 (1985).

Vieville, C., Z. Mouloungui, and A. Gaset, "Esterification of Oleic Acid by Methanol Catalyzed by p-Toluenesulfonic Acid and the Cation Exchange Resins K2411 and K1481 in Supercritical Carbon Dioxide," Ind. Eng. Chem. Res., 32, 2065 (1993).

Wang, M., J. M. Smith, and B. J. McCoy, "Kinetics of Coal Thermolysis with a Mixed Solvent of tert-Butyl Alcohol and Tetralin," Energy Fuels, 7, 78 (1993).

Wang, M., C. Zhang, J. M. Smith, and B. J. McCoy, "ContinuousMixture Kinetics of Thermolytic Extraction of Coal in Supercritical Fluid," AIChE J., 40, 131 (1994).

Warzinski, R. P., C. Lee, and G. D. Holder, "Supercritical-Fluid Solubilization of Catalyst Precursors: The Solubility and Phase Behavior of Molybdenum Hexacarbonyl in Supercritical Carbon Dioxide and Application to the Direct Liquefaction of Coal," J. Supercrit. Fluids, 5, 60 (1992).

Webley, P. A., J. W. Tester, and H. R. Holgate, "Oxidation Kinetics 
of Ammonia and Ammonia-Methanol Mixtures in Supercritical Water in the Temperature Range $530-700^{\circ} \mathrm{C}$ at 246 Bar," Ind. Eng. Chem. Res., 30, 1745 (1991).

Webley, P. A., H. R. Holgate, D. M. Stevenson, and J. W. Tester, "Oxidation Kinetics of Model Compounds of Metabolic Waste in Supercritical Water," SAE Tech. Paper 901333 (1990).

Webley, P. A., and J. W. Tester, "Fundamental Kinetics of Methane Oxidation in Supercritical Water," Energy Fuels, 5, 411 (1991).

Webley, P. A., and J. W. Tester, "Fundamental Kinetics of Methanol Oxidation in Supercritical Water," Supercritical Fluids: Science and Technology, K. P. Johnston and J. M. L. Penninger, eds., ACS Symp. Ser., No. 406, p. 259 (1989).

Weimer, P. J., C. T. Chou, W. M. Weston, and D. B. Chase, "Effect of Supercritical Ammonia on the Physical and Chemical Structure of Ground Wood," Proc. Biotech. Bioeng. Symp., No. 17, p. 5 (1986).

West, M. B., and M. R. Gray, "Pyrolysis of 1,3-Butanediol as a Model Reaction for Wood Liquefaction in Supercritical Water," Can. $J$. Chem. Eng., 65, 645 (1987).

Wheeler, J. C., and R. G. Petschek, "Anomalies in Chemical Equilibria Near Critical Points of Dilute Solutions," Phys. Rev. A, 28 , 2442 (1983).

Wightman, T. J., "Studies in Supercritical Wet Air Oxidation," MS Thesis, Univ. of California, Berkeley (1981).

Williams, A., "Combustion of Droplets of Liquid Fueis: A Review," Combust. Flame, 21, 1 (1973).

Wood, P. T., W. T. Pennington, and J. W. Kolis, "Synthesis of Novel Solid-State Compounds in Supercritical Solvents: Preparation and Structure of $\mathrm{K}_{2} \mathrm{Ag}_{12} \mathrm{Se}_{7}$ in Supercritical Ethylenediamine," $J$. Amer. Chem. Soc, 114, 9233 (1992).

Wood, P. T., W. T. Pennington, and J. W. Kolis, "Synthesis of $\mathrm{M}_{2} \mathrm{Ag}_{6} \mathrm{~S}_{4}(\mathrm{M}=\mathrm{Na}, \mathrm{K})$ in Supercritical Ethylenediamine Solvent: A Novel Solid Containing Unusual Closed-Shell Bonding," J. Chem. Soc., Chem. Commun., p. 235 (1993a).

Wood, P. T., W. T. Pennington, J. W. Kolis, B. Wu, and C. J. O'Connor, "Inorganic Synthesis in Supercritical Amines: Synthesis of $\left[\mathrm{W}_{4} \mathrm{~S}_{8}\left(\mathrm{H}_{2} \mathrm{NCH}_{2} \mathrm{CH}_{2} \mathrm{NH}_{2}\right)_{4}\right] \mathrm{S}$, Containing an Isolated Sulfide Ion," Inorg. Chem., 32, 129 (1993b).

Wu, B. C., M. T. Klein, and S. I. Sandler, "Reactions in and with Supercritical Fhids: Effect of Phase Behavior on Dibenzyl Ether Pyrolysis Kinetics," Ind. Eng. Chem. Res., 28, 255 (1989).

Wu, B. C., S. C. Paspek, M. T. Klein, and C. LaMarca, "Reactions in and with Supercritical Fluids," Supercritical Fluid Technology, Chap. 15, T. J. Bruno and J. F. Ely, eds, CRC Press, Boca Raton, FL (1991a).

Wu, B. C., M. T. Klein, and S. I. Sandler, "Solvent Effects on Reactions in Supercritical Fluids," Ind. Eng. Chem. Res., 30, 822 (1991b).

Wu, B. C., M. T. Klein, and S. I. Sandler, "Influence of Superctitical Fluid Solvent Density on Benzyl Phenyl Ether Pyrolysis: Indications of Diffusion Limitations," Energy Fuels, 5, 453 (1991c).

Xu, X., C. De Almeida, and M. J. Antal, Jr., "Mechanism and Kinetics of the Acid-Catalyzed Dehydration of Ethanol in Superctitical Water," J. Supercrit. Fluids, 3, 228 (1990).

Xu, X., C. De Almeida, and M. J. Antal, Jr., "Mechanism and Kinetics of the Acid-Catalyzed Formation of Ethene and Diethyl Ether from Ethanol in Supercritical Water," Ind. Eng. Chem. Res., 30, 1478 (1991).

Yagi, Y., S. Saito, and H. Inomata, "Tautomerization of 2,4-Pen- tanedione in Supercritical $\mathrm{CO}_{2}, " J$. Chem. Eng. Japan, 26, 116 (1993).

Yalpani, M., "Supercritical Fluids: Puissant Media for the Modification of Polymers and Biopolymers," Polymer, 34, 1102 (1993).

Yang, H. H., and C. A. Eckert, "Homogeneous Catalysis in the Oxidation of p-Chlorophenol in Supercritical Water," Ind. Eng. Chem. Res., 27, 2009 (1988).

Yogo, T., S. Naka, and S. Hirano, "Synthesis and Properties of Magnetite-Dispersed Carbon by Pressure Pyrolysis of Divinylbenzenevinylfertocene with Water," J. Mat. Sci. Lett., 24, 2115 (1989).

Yokota, K., and K. Fujimoto, "Supercritical-Phase Fischer-Tropsch Synthesis Reaction: 2. The Effective Diffusion of Reactant and Products in the Supercritical-Phase Reaction," Ind. Eng. Chem. Res., 30, 95 (1991).

Yokota, K., Y. Hanakata, and K. Fujimoto, "Supercritical Phase Fischer-Tropsch Synthesis Reaction," Natural Gas Conversion: Studies in Surface Science and Catalysis, A. Holmen, K.-J. Jens, and S. Kolboe, eds., Elsevier, Amsterdam, p. 289 (1991).

Yokota, K., Y. Hanakata, and K. Fujimoto, "Supercritical Phase Fischer-Tropsch Synthesis Reaction," Fuel, 68, 255 (1989).

Yoshimura, Y., and Y. Kimura, "Solvent-Density Dependence of the Unimolecular Reaction Rate: Dissociation Reaction of 2-Methyl2-Nitrosopropane Dimer in Carbon Dioxide from Gas to Liquid States," Chem. Phys. Lett., 181, 517 (1991).

Yu, D., M. Aihara, and M. J. Antal, Jr., "Hydrogen Production by Steam Reforming Glucose in Supercritical Water," Energy Fuels, 7, 574 (1993).

Yu, Z.-R., S. S. H. Rizvi, and J. A. Zollweg, "Enzymatic Esterification of Fatty Acid Mixtures from Milk Fat and Anhydrous Milk Fat with Canola Oil in Supercritical Carbon Dioxide," Biotech. Prog., 8, 508 (1992).

Yürüm, Y., R. Kramer, and M. Levy, "Thermochemical Reactions in Subcritical and Supercritical Interaction Between Mishor Rotem Oil Shale and Toluene," Thermochim. Acta, 105, 51 (1986).

Yürüm, Y., H. Özyörük, N. Altuntas, and H. Gülce, "Identification of Organic Sulfur Compounds in Supercritical Extracts of Beypazari Lignite Using Deconvoluted Differential Pulse Polarograms," Energy \& Fuels, 7, 620 (1993).

Zagrobelny, J., and F. V. Bright, "In Situ Studies of Protein Conformation in Supercritical Fluids: Trypsin in Carbon Dioxide," Biotech. Prog., 8, 421 (1992a).

Zagrobelny, J, and F. V. Bright, "Influence of Solute-Fluid Clustering on the Photophysics of Pyrene Emission in Supercritical $\mathrm{C}_{2} \mathrm{H}_{4}$ and $\mathrm{CF}_{3} \mathrm{H}, " J$. ACS, 114, 7821 (1992b).

Zagrobelny, J., and F. V. Bright, "Probing Solute-Entrainer Interactions in Matrix-Modified Supercritical $\mathrm{CO}_{2}, " J . A C S, 115,701$ (1993).

Zagrobeiny, J., T. A. Betts, and F. V. Bright, "Steady-State and Time-Resolved Fluorescence Investigations of Pyrene Excimer Formation in Supercritical $\mathrm{CO}_{2}, " J . A C S, 114,5249$ (1992).

Zhang, C., J. M. Smith, and B. J. McCoy, "Kinetics of Supercritical Fluid Extraction of Coal; Physical and Chemical Processes," Supercritical Fluid Engineering Science: Fundamentals and Applications, E. Kiran and J. F. Brennecke, eds., ACS Symp. Ser., No. 514, p. 363 (1993).

Manuscript received Apr. 6, 1994, and revision received Aug. 17, 1994. 